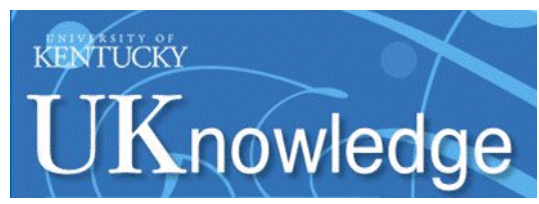

University of Kentucky

UKnowledge

Theses and Dissertations--Earth and

Environmental Sciences

2019

\title{
TEMPORAL AND SPATIAL VARIABILITY IN GROUNDWATER FLOW AND CHEMISTRY ALONG THE CUMBERLAND RIVER, ARTEMUS, KENTUCKY
}

Amanda Rachelle Sherman

University of Kentucky, amanda.sherman.369@gmail.com

Author ORCID Identifier:

(iD) https://orcid.org/0000-0002-8281-7166

Digital Object Identifier: https://doi.org/10.13023/etd.2019.220

Right click to open a feedback form in a new tab to let us know how this document benefits you.

\section{Recommended Citation}

Sherman, Amanda Rachelle, "TEMPORAL AND SPATIAL VARIABILITY IN GROUNDWATER FLOW AND CHEMISTRY ALONG THE CUMBERLAND RIVER, ARTEMUS, KENTUCKY" (2019). Theses and Dissertations--Earth and Environmental Sciences. 68.

https://uknowledge.uky.edu/ees_etds/68

This Master's Thesis is brought to you for free and open access by the Earth and Environmental Sciences at UKnowledge. It has been accepted for inclusion in Theses and Dissertations--Earth and Environmental Sciences by an authorized administrator of UKnowledge. For more information, please contact UKnowledge@lsv.uky.edu. 


\section{STUDENT AGREEMENT:}

I represent that my thesis or dissertation and abstract are my original work. Proper attribution has been given to all outside sources. I understand that I am solely responsible for obtaining any needed copyright permissions. I have obtained needed written permission statement(s) from the owner(s) of each third-party copyrighted matter to be included in my work, allowing electronic distribution (if such use is not permitted by the fair use doctrine) which will be submitted to UKnowledge as Additional File.

I hereby grant to The University of Kentucky and its agents the irrevocable, non-exclusive, and royalty-free license to archive and make accessible my work in whole or in part in all forms of media, now or hereafter known. I agree that the document mentioned above may be made available immediately for worldwide access unless an embargo applies.

I retain all other ownership rights to the copyright of my work. I also retain the right to use in future works (such as articles or books) all or part of my work. I understand that I am free to register the copyright to my work.

\section{REVIEW, APPROVAL AND ACCEPTANCE}

The document mentioned above has been reviewed and accepted by the student's advisor, on behalf of the advisory committee, and by the Director of Graduate Studies (DGS), on behalf of the program; we verify that this is the final, approved version of the student's thesis including all changes required by the advisory committee. The undersigned agree to abide by the statements above.

Amanda Rachelle Sherman, Student

Dr. Alan Fryar, Major Professor

Dr. Ed Woolery, Director of Graduate Studies 
TEMPORAL AND SPATIAL VARIABILITY IN

GROUNDWATER FLOW AND CHEMISTRY ALONG THE

CUMBERLAND RIVER, ARTEMUS, KENTUCKY

Thesis

A thesis submitted in partial fulfillment of the requirements for the degree of Master of Science in the College of Arts and Sciences at the University of Kentucky

By

Amanda Rachelle Sherman

Lexington, Kentucky

Director: Dr. Alan Fryar, Associate Professor of Earth and

Environmental Sciences

Lexington, Kentucky

2019

Copyright (C) 2019 Amanda Rachelle Sherman 


\section{Abstract of Thesis \\ TEMPORAL AND SPATIAL VARIABILITY IN GROUNDWATER FLOW AND CHEMISTRY ALONG THE CUMBERLAND RIVER, ARTEMUS, KENTUCKY}

Groundwater in the Kentucky Appalachian region is constrained by physiography and lithology. Lithostratigraphy, groundwater flow, and chemistry were delineated in the alluvial aquifer along the Cumberland River at H.L. Disney Training Center. To assess groundwater-river interactions and water quality, 11 monitoring wells were installed and sampled quarterly, plus the river and an existing bedrock well. Analytical results were evaluated for temporal and spatial trends. Collected soil cores were analyzed for bulk chemistry and grain size. Solute speciation and saturation indices were calculated and hydraulic conductivity estimated from grain-size analyses. Pumping and slug tests were performed to estimate hydraulic conductivity and hydraulic head was monitored using logging transducers for river stage comparison.

Site lithology consists of loamy soils underlain by silty clay, transitioning downward to clayey-fine sands on friable sandstone/shale. Alluvium becomes finergrained and has lower hydraulic conductivities with proximity to the river $\left(10^{-9}-10^{-2} \mathrm{~cm} / \mathrm{s}\right)$. Meteoric recharge drives local groundwater flow from ridges toward rivers. Hydraulic head fluctuates with stage and temporary gradient reversals occur. Groundwater does not appear to be impacted by current land use. Wells have elevated iron and manganese concentrations; post-treatment, the alluvial aquifer may provide sufficient quality and rates of water to support onsite military activities.

KEYWORDS: Water Resources, Hydrogeochemistry, Hydraulic Conductivity, Eastern Kentucky, Alluvial Aquifer

Amanda Rachelle Sherman

(Name of Student)

May 14, 2019

Date 
TEMPORAL AND SPATIAL VARIABILITY IN GROUNDWATER FLOW AND CHEMISTRY ALONG THE CUMBERLAND RIVER, ARTEMUS, KENTUCKY

\author{
By
}

Amanda Rachelle Sherman

Dr. Alan Fryar

Director of Thesis

Dr. Ed Woolery

Director of Graduate Studies

May 14, 2019

Date 


\section{Dedication}

"Make you the world a bit better or more beautiful because you have lived in it." $\sim$ Edward W. Bok 


\section{Acknowledgements}

The following thesis benefited from the insights and direction of several people. First, my Thesis Chair, Dr. Alan Fryar, exemplifies the high-quality professionalism to which I aspire. In addition, Dr. Fryar provided guidance and evaluation at every stage of the thesis process. Next, I wish to thank my Thesis Committee, Dr. Junfeng Zhu and Dr. Andrea Erhardt. Each provided insights that guided and challenged my thinking, improving the finished product.

Faculty and staff within the Department of Earth and Environmental Sciences were welcoming and supportive in my research and academic pursuits. Thank you for providing the opportunity and means to succeed. A special thanks to Department undergraduates Colin Marshall, Matthew Cecil, and Ryan Parks, and the STEAM Academy intern, Grant Walles, for their assistance with field and laboratory work.

In addition to the technical and instrumental assistance above, I received equally important assistance from family and friends. My husband, Jason Sherman, provided ongoing support throughout the thesis process, as well as giving me the courage to try and never losing faith. Finally, my deepest gratitude to everyone at the Kentucky Army National Guard, Environmental Office for sharing institutional knowledge and resources. I would also like to thank them for their financial support and confidence in the Earth and Environmental Sciences Department, without which this thesis would not have been possible. I would especially like to thank Ricky French, Bob Middleton, Greg Disney, and Donovan Sherratt. 
Acknowledgements.........................................................

List of Tables...............................................................

List of Figures............................................................

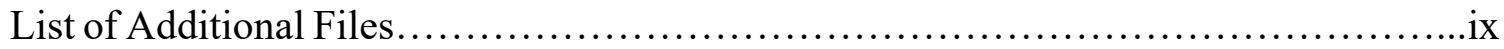

Chapter 1: Introduction....................................................

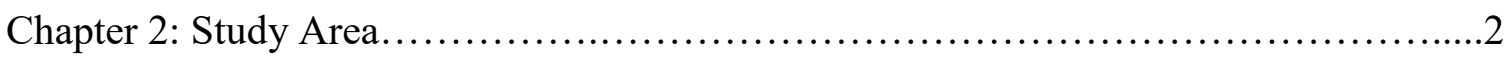

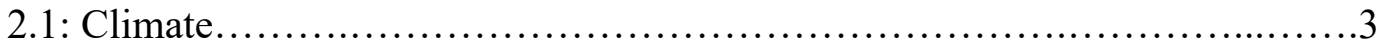

2.2: Land Use....................................................

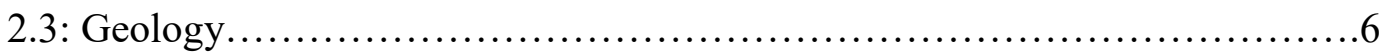

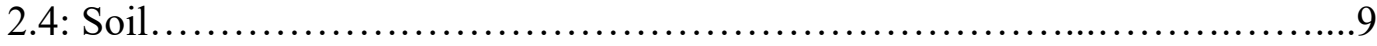

2.5: Hydrogeology ................................................... 10

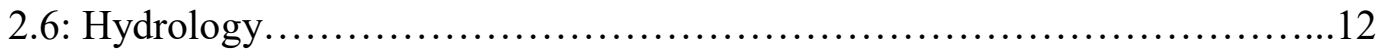

2.7: Previous Work................................................ 15

Chapter 3: Methods...................................................... 16

3.1: Water-Quality Assessment......................................... 16

3.1.1: Monitoring Well Installation...............................16

3.1.2: Water Sampling........................................... 19

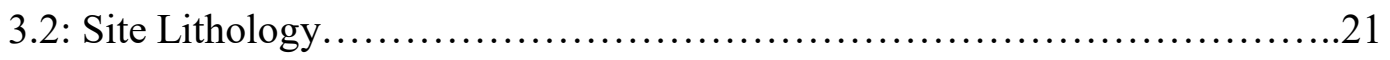

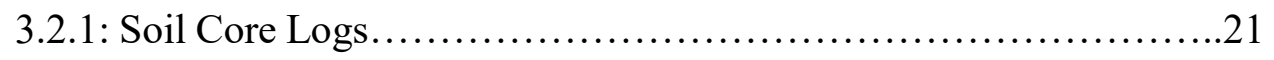

3.2.2: Grain Size Analysis.........................................22

3.2.3: Carbon Content.........................................23

3.2.4: Mineral Composition......................................24

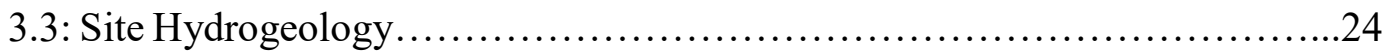

3.3.1: Hydraulic-Head Monitoring.................................24

3.3.2: Cumberland River Hydrographs and Precipitation.................25 
3.3.3: Hydraulic Conductivity....................................25

3.4: Monitoring Well Abandonment........................................26

Chapter 4: Results and Discussion............................................27

4.1: Water-Quality Assessment..........................................27

4.2: Site Lithology .................................................. 78

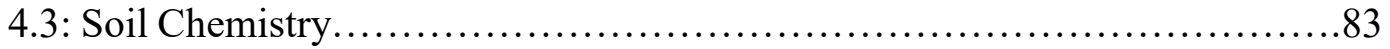

4.4: Site Hydrogeology ..............................................95

4.4.1: Groundwater Flow........................................95

4.4.2: Hydraulic Conductivity..................................104

Chapter 5: Conclusions and Recommendations................................... 110

5.1: Conclusions................................................. 110

5.2: Recommendations................................................111

References.............................................................. 113

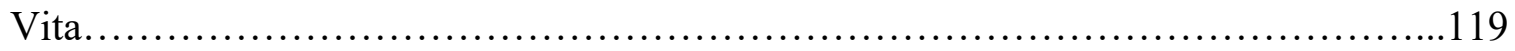




\section{List of Tables}

Table 1: USDA Custom Soil Resource Report Table...............................10

Table 2: HLDTC Well Locations and Cumberland River Sample Site...................19

Table 3: HLDTC Water-Quality Parameters..................................... 30

Table 4: HLDTC Major Cations Analytical Results..................................33

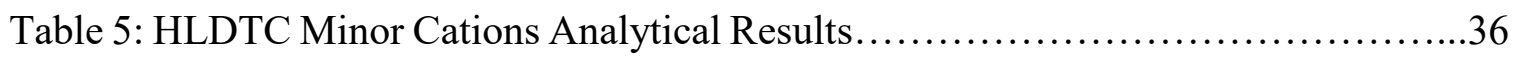

Table 6: HLDTC Metalloids Analytical Results..................................41

Table 7: HLDTC Anion and Nutrient Analytical Results............................46

Table 8: HLDTC Charge-Balance Error Calculations (CBE\%) .......................58

Table 9: HLDTC Mineral Saturation Indexes (SI) ..............................60

Table 10: HLDTC VOC Results for Detected Analytes............................ 70

Table 11: HLDTC Isotope Analytical Results................................. 72

Table 12: HLDTC Pumping Test Time Series Analytical Results.....................78

Table 13: HLDTC Hydrometer/Sieve Grain Size Results..........................82

Table 14: HLDTC Soil Carbon Analytical Results....................................84

Table 15: HLDTC Soil XRF Analytical Results for Major Elements (wt.\%).............88

Table 16: HLDTC Soil XRF Analytical Results for Minor Elements (wt.\%)............91

Table 17: HLDTC HydrogeoSieve XL Hydraulic Conductivity (K) Estimation...........105

Table 18: HLDTC Well Capacity Hydraulic Conductivity, Slug and Pump Tests.........109 


\section{List of Figures}

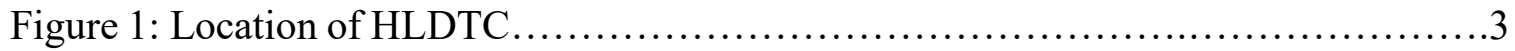

Figure 2: Map of soil types, cropped fields, wetlands, and training areas at

HLDTC .......................................................4

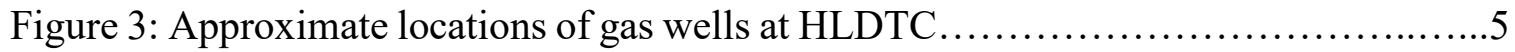

Figure 4: Geologic map of study area ................................................

Figure 5: Custom Soil Resource Report Map for study area.............................9

Figure 6: HLDTC lies within the southern hydrologic Kanawha section...................11

Figure 7: Cumberland River basin and approximate site location.......................13

Figure 8: FEMA flood map for HLDTC ......................................... 14

Figure 9: Approximate location of nearest USGS gaging stations upstream and

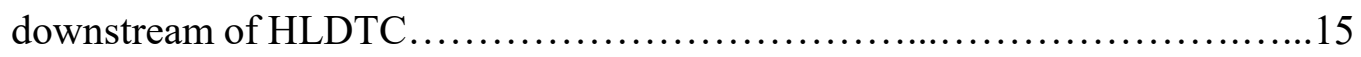

Figure 10: Location of monitoring wells, dry well locations, existing wells, and Cumberland River sample point......................................... 17

Figure 11: Generalized monitoring well construction sketch for HLDTC..........................18

Figure 12: Air (24-hr moving average) and groundwater temperatures at HLDTC from

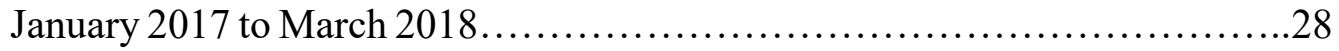

Figure 13: Quarterly Piper diagrams for HLDTC from January 2017 to January 2018 ......54

Figure 14: Stable isotopes of groundwate at HLDTC and river water from January 2017 to

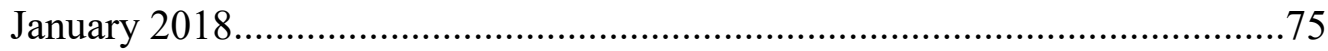

Figure 15: HLDTC quarterly well monitoring isotope results for $\delta^{18} \mathrm{O}$ and $\delta^{2} \mathrm{H}$ from January 2017 to March 2018..............................................................................

Figure 16: HLDTC monitoring well construction diagram with representative lithologic units.

Figure 17: HLDTC Al:Si ratios in soil cores as a function of depth.

Figure 18: HLDTC Mn and Fe concentrations in soil core samples measured by majorand minor-element XRF analysis as a function of depth. 
Figure 19: HLDTC water-table map from January 2017 to March 2018. Contours are in meters above sea level...............................................96

Figure 20: HLDTC hydrographs for MW-6 and MW-7 with interpreted Cumberland River stage and 24-hr precipitation totals January 2017 to March 2018......102

Figure 21: HLDTC hydrographs detailing gradient reversal during February 2018 in MW-6 and MW-7 with interpreted Cumberland River stage and 24-hr precipitation..................................................... 100 
Core $\log$ MW No............................................. ZIP 9,047 KB)

Core Photos MW No................................................ZIP 9,576 KB)

HydrogeoSieve XL Grain Size MW No..............................(ZIP 1,573 KB)

HydrogeoSieveXL Hydraulic MW No..............................(ZIP 3,104 KB)

Hydrometer-Sieve Grain Size Analysis..............................(ZIP 336 KB)

Kentucky Well Construction Development Plugging Records............(ZIP 18,230 KB)

KGS Analytical Reports.......................................(ZIP 620 KB)

McCoy VOC Analytical Reports.................................(ZIP 1,235 KB)

UW Isotope Analytical Reports.................................(ZIP 7,825 KB) 


\section{Introduction}

In October 2010, the Honorable Katherine Hammack, Assistant Secretary of the Army for Installations, Energy and Environment, announced the Army Net Zero Initiative. Net Zero is based on combining sustainability with emerging best practices to better manage energy, water, and waste at Army installations. The concept originated from Federal mandates, including Executive Order 13514, the Energy Policy Act of 2005, and the Energy Independence and Security Act of 2007. The Initiative is sustained by recognition that resource management improves mission effectiveness and creates resilient installations (McMordie Stoughton et al., 2013).

The Net Zero water strategy strives to balance water availability and use to ensure a sustainable water supply for the future. This is of increasing importance as water scarcity is a growing global concern (McMordie Stoughton et al., 2013). Therefore, an installation exemplifying Net Zero water limits the use of freshwater resources and returns withdrawn water to the same watershed. This is done in an effort to limit impacts on groundwater and surface water resources.

In September 2016, a Memorandum of Agreement (MOA) was entered into by the Kentucky Department of Military Affairs and the University of Kentucky, Department of Earth and Environmental Sciences. The Kentucky National Guard (KNG) sought the MOA to assess potential potable and non-potable water resources on the CSM Harold L. Disney Training Center (HLDTC) located in Knox County, Kentucky (KY). The MOA outlines five project objectives:

1. Map the water table across the site.

2. Determine seasonal fluctuations in the depth of the water table. 
3. Define directions and rates of groundwater flow.

4. Assess groundwater interactions with the Cumberland River.

5. Characterize seasonal variations in groundwater and surface water chemistry, including consideration of impacts of land use and training activities on water quality.

Conclusions from the five aforementioned objectives will aid the $\mathrm{KNG}$ in supporting the water Net Zero Initiative at HLDTC. Per the MOA, the efforts involved to document the methods, summarize data, and present the findings for the five objectives serve as the basis for a master's thesis in Geological Sciences. As such, the following three hypotheses have been developed to reflect the aforementioned objectives:

1. Meteoric recharge will be the predominant influence on water-table elevations, which will fluctuate seasonally from highest in the summer/fall to lowest in the winter/spring. However, within the riparian zone along the Cumberland River, elevated river stage events will result in a temporally and spatially elevated water table.

2. Groundwater flow will follow local topography from the toe of the ridge along the southern boundary of HLDTC northward toward the Cumberland River.

3. Groundwater chemistry will reflect processes during recharge in the soil zone and limited water-rock interactions in clastic sediments. Hydraulic-gradient reversals during elevated river stage will affect groundwater chemistry locally within the riparian zone. 


\section{Study Area}

HLDTC is located in southeastern Knox County, $\sim 0.5 \mathrm{~km}$ southeast of the town of Artemus, as shown on the U.S. Geological Survey (USGS) Artemus Quadrangle geologic map (Figure 1). The study area consists of $\sim 234.7$ ha (Hettinger, C., KNG, personal communication, October 13, 2015) and is bounded on the east, north, and west by the Cumberland River. The ridgeline to the south roughly forms the southern boundary (Hettinger, C., KNG, personal communication, February 19, 2016).

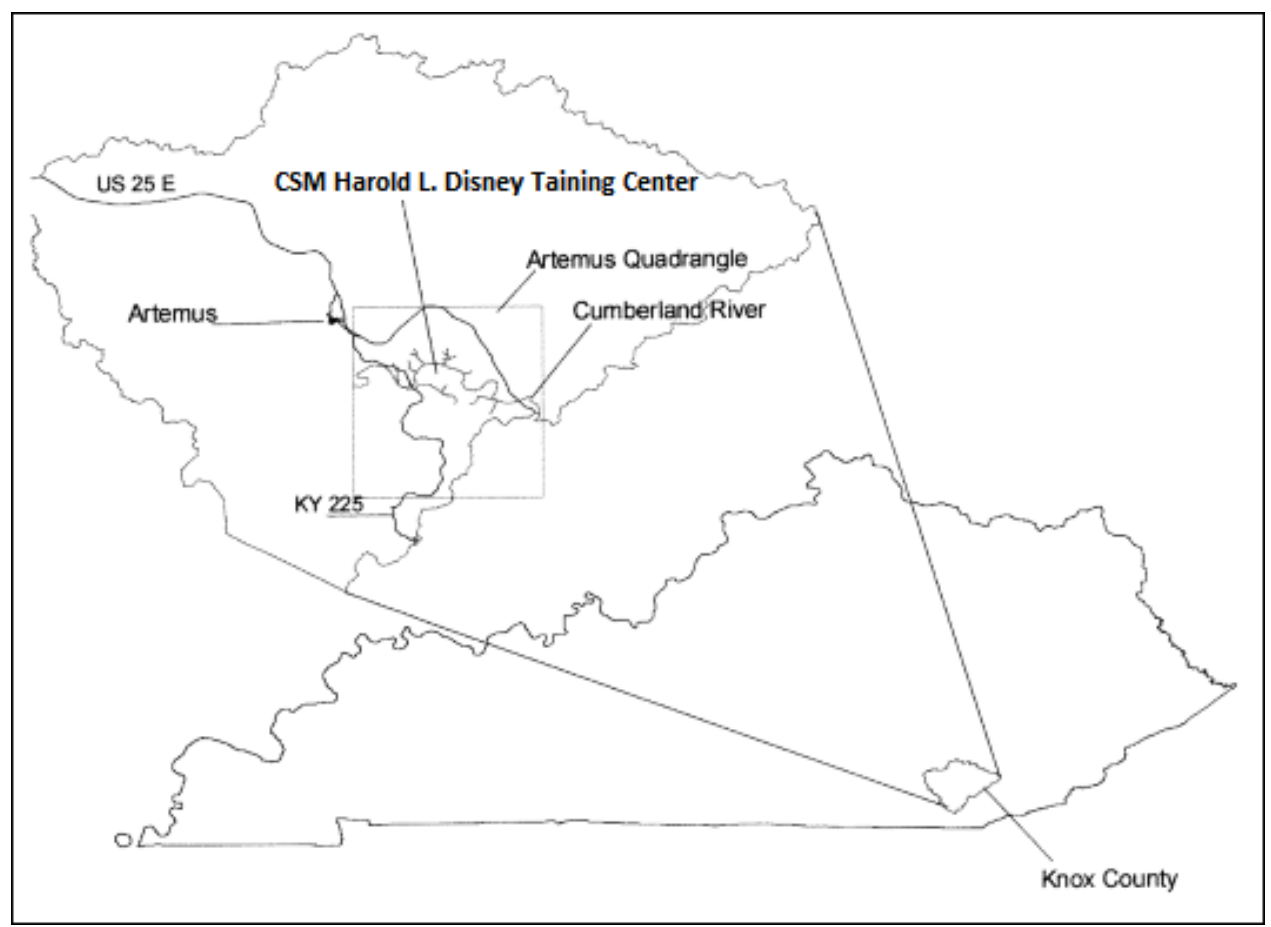

Figure 1: Location of HLDTC (modified from Figure 1, Cumbie et al., 2004).

\subsection{Climate}

The Koeppen Climate Classification subtype for the study area is Cfa (humid subtropical). The average annual temperature is $13.3^{\circ} \mathrm{C}$, with July being the warmest month (mean $24.2^{\circ} \mathrm{C}$ ) and January the coldest (mean $1.55^{\circ} \mathrm{C}$ ). The average annual precipitation is $127.5 \mathrm{~cm}$, with the highest precipitation in July (mean $12.9 \mathrm{~cm}$ ) and the lowest in October (mean $7.4 \mathrm{~cm}$ ). There are on average 124.3 days of rain, with March as the wettest month 
(mean 11.8 days) and October the driest (mean 8.0 days). There is an annual average of $22.8 \mathrm{~cm}$ of snow, most of it occurring in January (mean $9.1 \mathrm{~cm}$ ) (Weatherbase, 2017).

\subsection{Land Use}

HLDTC is predominantly used by the KNG for weekend and annual (2-week) training. To support the training mission, the training center has a confidence and rappel tower; an engagement skills trainer; military operations on urban terrain sites; and training areas. The support buildings include a classroom; four 40-person capacity barracks, including a shower house with latrines; an engagement skills training building; and two pavilions (https://whfrtc.ky.gov/hldtc/Pages/default.aspx). HLDTC also has an active Federal agricultural lease for $\sim 67.9$ ha of its 234.7 ha. Hay is cultivated on $\sim 42.1$ ha and row crops on the remaining 25.9 ha (Figure 2).

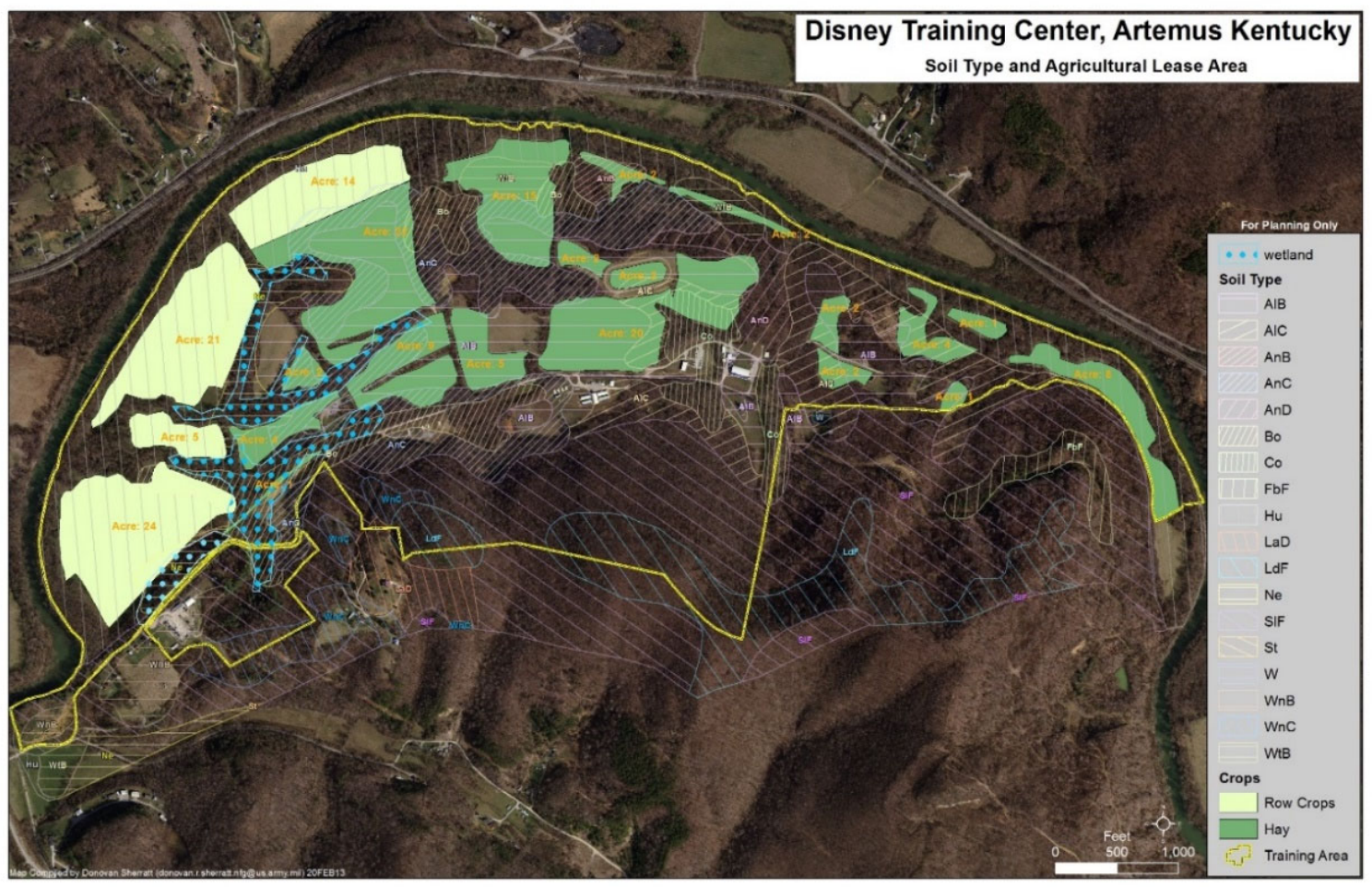

Figure 2: Map of soil types, cropped fields, wetlands, and training areas at HLDTC (Sherratt, 2013). 
Onsite mineral resources include coal and natural gas. In the past, the Lily coal bed located near the base of the ridge on the eastern portion of the property was mined locally by horizontal augering (Cumbie et al., 2004). As of June 2004, there were 14 gas wells onsite with six still in production. The depths of these wells ranged from 322.1 to $687 \mathrm{~m}$ below land surface (bls) (Figure 3 ). The Himyar gas field, located $\lesssim 1.6 \mathrm{~km}$ east of HLDTC, produces gas from stratigraphic traps in the Silurian "Big Six" on the Flat Lick Anticline crest and flanks (Figure 4). Gas rights within the study area are retained by Vinland Energy and Delta Gas (Hettinger, C., personal communication, February 19, 2016).

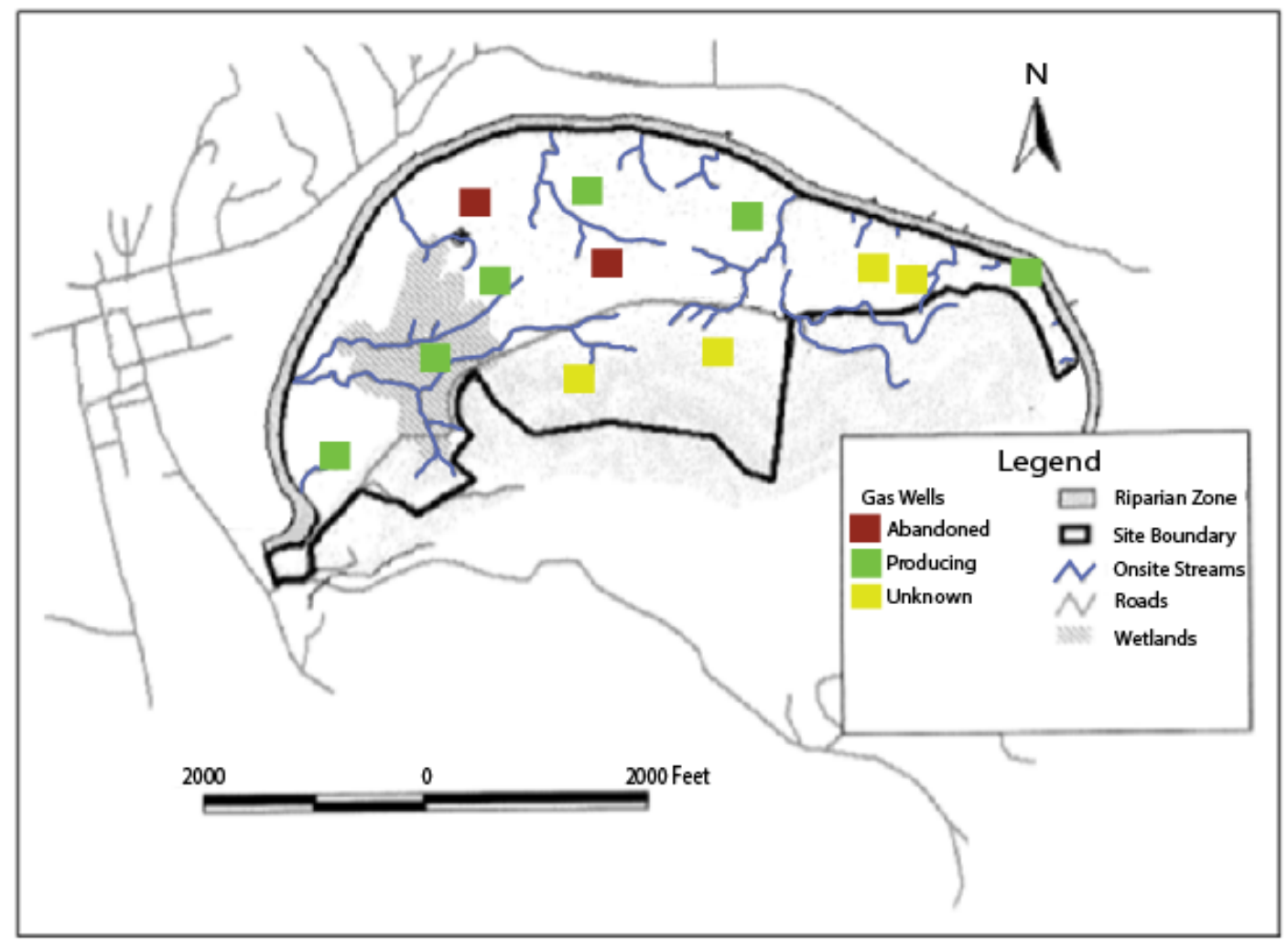

Figure 3: Approximate locations of gas wells at HLDTC (modified from Figure 2, Cumbie et al., 2004). 


\subsection{Geology}

The study site is located in the Appalachian Mountains, which were formed by orogenic events along a passive continental margin. The detritus in most sandstones can be ascribed to sources based on provenance types. The Appalachians are considered a passivemargin, recycled orogeny, where the sandstone sources are deformed and uplifted stratal sequences in subduction zones, along collisional orogens or within foreland fold-thrust belts. Typical alluvial sands are composed of recycled sedimentary rock and have intermediate quartz content, a high quartz to feldspar ratio, and an abundance of sedimentary-metasedimentary lithic fragments (Dickinson and Suczek, 1979).

The study area is located on the southwest rising limb of the Flat Lick Anticline. The crest of the anticline plunges predominantly west-northwest to east-southeast. Across the study area, the structure elevation changes from $427 \mathrm{~m}$ to $378 \mathrm{~m}$ above sea level (asl). Most of the study area is situated on the Cumberland River valley Quaternary alluvium. The alluvium is composed of flood plain (silt, clay, sand, and gravel) and low-level terrace (sand, silt, gravel, and clay) deposits with a thickness of $0-15.2 \mathrm{~m}$. The boundary between the two deposits is generally poorly defined, gradational, and coincides with the limit of the 5- to 10-year flood crests (Rice, 1974).

The southern boundary of the property traverses the northern slope of a bedrock ridge composed of the Pennsylvanian Breathitt Formation (Fm.) (Chesnut, 1992). The lithology of the lower Breathitt Fm. within Knox County is shale, siltstone, sandstone, coal, and conglomerate, while the middle Breathitt Fm. is sandstone, shale, siltstone, coal, and limestone (Schweitzer, 2015). Locally, the Breathitt Fm. is subdivided into five subunits; the lower three are exposed in the study area. The lowest Breathitt Fm. subunit is exposed 
along the base of the ridge from the center of the study area eastward (Rice, 1974). That unit is overlain by the Lily coal bed, which is exposed in the eastern portion of the study area (Cumbie et al., 2004). The second-lowest Breathitt Fm. subunit is atop the Lily coal bed and comprises most of the ridge. It is exposed along the entire southern boundary of the study area. The Blue Gem coal bed overlies the second-lowest subunit. The third-lowest Breathitt Fm. subunit is above the Blue Gem coal bed and is exposed on the ridge crests. Not exposed within the study area are the fourth- and fifth-lowest Breathitt Fm. subunits and the Tertiary (?) and Quaternary high-level fluvial deposits, which lie above the Breathitt Fm. subunits and below the alluvium. The lithology of the high-level fluvial deposits is sand, silt, clay, and gravel with a thickness of 0-4.6 m (Rice, 1974). 

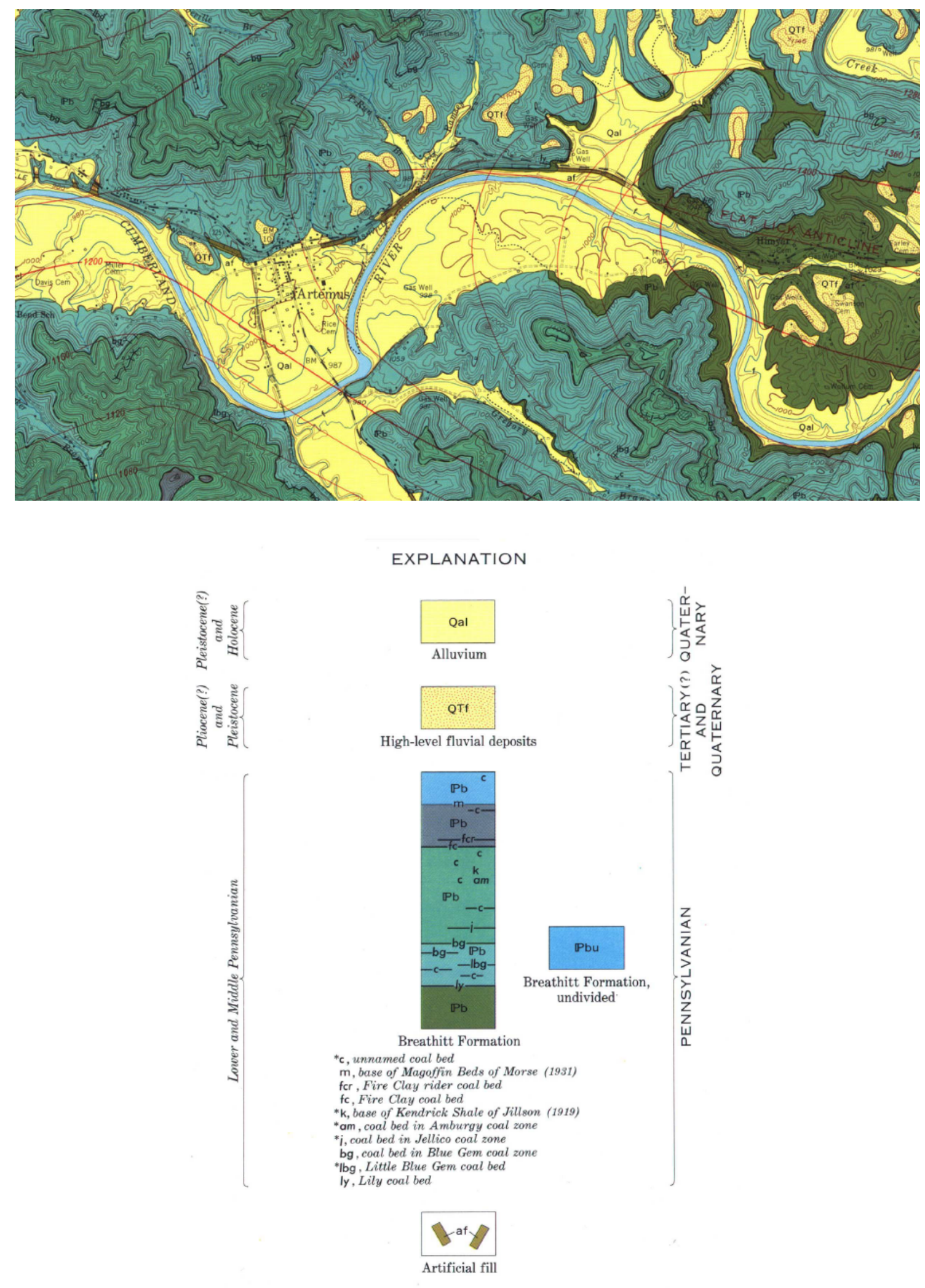

*Shown locally by outerop or adit symbol only

Figure 4: Geologic map of study area (modified from Rice, 1974). 


\subsection{Soils}

A Custom Soil Resource Report for Knox and Eastern Part of Whitley Counties, Kentucky for CSM Harold L. Disney was created via the Web Soil Survey portal available through the United States Department of Agriculture (USDA) Natural Resources Conservation Service website (Figure 5). The report concludes that most of the study area soils are silt loams, with some loam and complex (two or more soils intricately mixed) present (USDA, 2017). The soil symbol, names, and brief description are provided in Table 1.

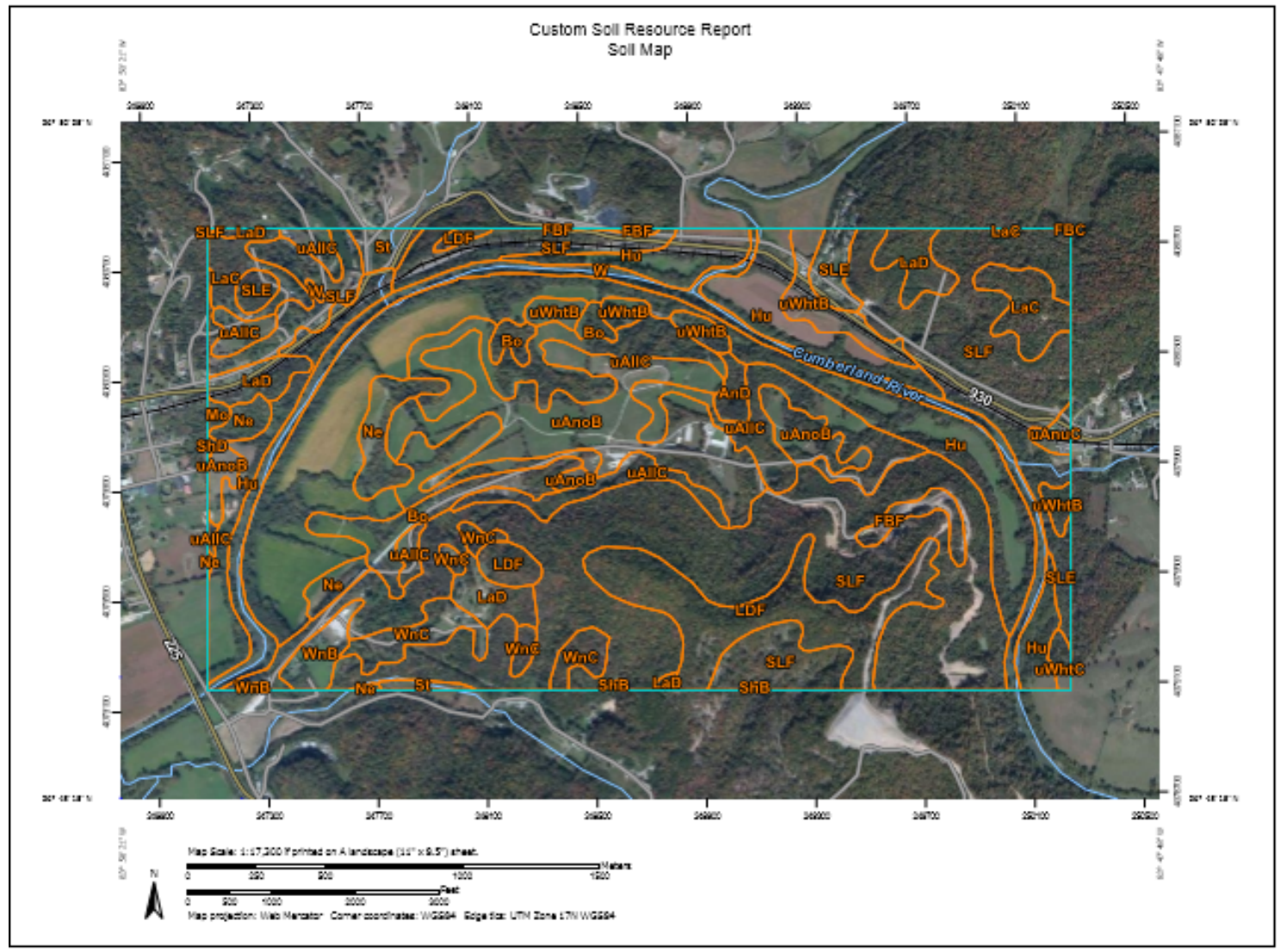

Figure 5: Custom Soil Resource Report Map for study area (USDA, 2017). 
Table 1. USDA Custom Soil Resource Report Table

\begin{tabular}{lll}
\hline Symbol & Name & Description \\
\hline $\mathrm{Hu}$ & Huntington silt loam & Occasionally flooded, well drained \\
$\mathrm{Ne}$ & Newark silt loam & Occasionally flooded, somewhat poorly \\
& drained \\
$\mathrm{Bo}$ & Bonnie silt loam & Frequently flooded, very poorly drained \\
uWhtB & Whitley silt loam & 2 to $6 \%$ slopes, rarely flooded \\
uAllC & Allegheny loam & 6 to $12 \%$ slopes, well drained \\
uAnoB & Allegheny-Cotaco complex & 2 to $6 \%$ slopes, well drained \\
\hline
\end{tabular}

\subsection{Hydrogeology}

The study area lies within the southern hydrologic Kanawha section, which consists of highly dissected areas with narrow valleys and steep-sided ridges (Figure 6). Most wells are drilled into valley bottoms and tend to yield enough water for domestic use. Two sources of groundwater exist in the Kanawha section: alluvium and the Breathitt Fm. (subarea 1) (Kilburn et al., 1962).

The alluvium forms the floodplains of the Kanawha and underlies terraces. Most dug wells in the alluvium yield $>100$ gallons/day ( $380 \mathrm{~L} /$ day), whereas screened wells drilled into sandy material with sufficient saturated thickness can yield $>500$ gallons/day (1900 L/day) (Kilburn et al., 1962). The water quality is generally soft to moderately hard and may contain large amounts of iron at depth (Carey and Stickney, 2005).

The Breathitt Fm. underlies valleys and forms hills and ridges. Hilltops and ridges are commonly capped by sandstone, while shales form wide valleys and gentle to moderate hill slopes (Carey and Stickney, 2005). The Breathitt Fm. yields > 500 gallons/day (1900 L/day) to almost half the wells on hillsides and smaller amounts to wells on hilltops. Most water is yielded from near-vertical joints and openings along bedding planes in sandstone, shale, and coal (Kilburn et al., 1962). Water quality is highly variable (Carey and Stickney, 2005). 


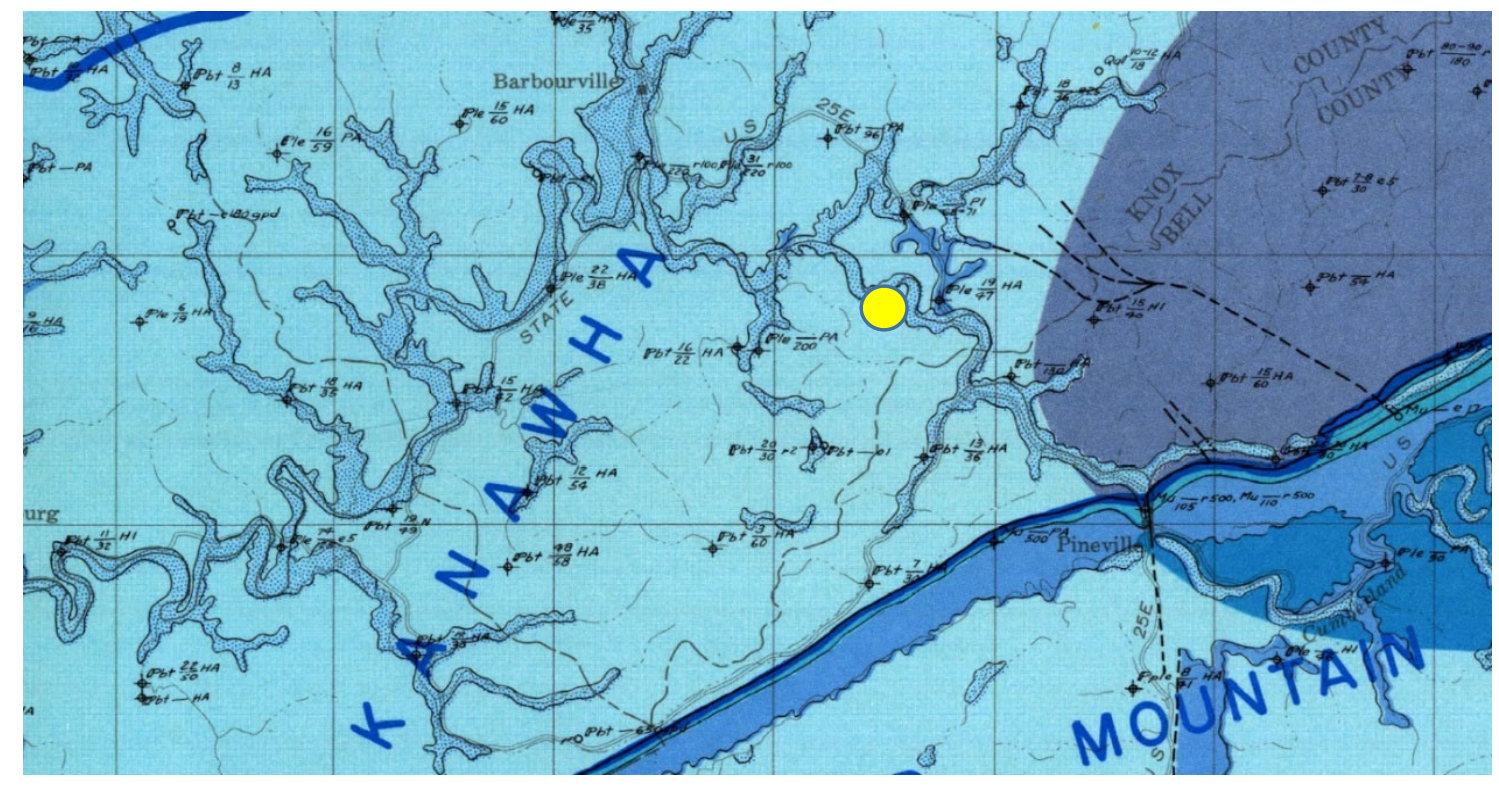

KANAWHA SECTION

The Kanawha section is a highly dissected area characterized by narrow valleys and irregular steep-sided ridges. Although shale is the predominant rock type, beds of sandstone form resistant ledges in the sides and on the tops of hills, and underlie some of the broad valleys. Because most of the people live along the streams, nearly all valleys. Because most of the people live along the streams, nearly all
wells are drilled in the valley bottoms. Rocks in this area generally wells are drilled in the valley bottoms. Rock
yield ample supplies of water for domestic use

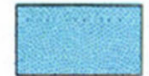

Water in alluvium

Dug wells.-Nearly all wells in the alluvium in this area are dug. Most dug wells are adequate for a minimum domestic supply. A few are adequate for a modern domestic supply. Water obtained from most dug wells in this area is moderately hard and contains noticeable amounts of iron.

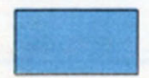

Water in the Lee formation (subarea 2)

Drilled wells.-Most wells drilled in valley bottoms are adequate for a modern domestic supply. Nearly all wells in valley bottoms are ade-

quate for a minimum domestic supply.
Fewer than half the wells drilled on hillsides are adequate for a modern domestic supply. Nearly all wells on hillsides are adequate for a minimum domestic supply.

Fewer than three-quarters of the wells drilled on hilltops and ridges are adequate for a minimum domestic supply. About a third of the supply.

supply. where it exceeds 500 feet in thickness may yield enough water for small municipal or industrial supplies.

Most water in this area is moderately hard and contains noticeable amounts of iron. Salty water may be found, in places in this area, at depths less than 100 feet. Nearly all wells tapping the Lee formation yield salty water where the Lee lies beneath the Breathitt formation

and below the principal drainage.
Dug wells.-Most wells dug in valley bottoms are adequate for a minimum domestic supply. A few wells dug in valley bottoms are adequate for a modern domestic supply.

g hills are adequate for a minimum A few wells dug on hills are adequate for a modern domestic supply

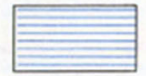

Salty water

Drilled wells.-Salty water may be found in wells drilled into the Breathitt formation or Lee formation in this area less than 100 feet below the level of the principal valley bottoms

\section{Water in the Breathitt formation (subarea 1)}

Drilled wells.-Most wells drilled in valley bottoms are adequate for a modern domestic supply. Nearly all wells in valleys are adequate for minimum domestic supply.

Fewer than half the wells drilled on hillsides are adequate for a modern domestic supply. More than three-quarters of the wells on Willes are adequate for a minimum domestic supply.

Wells on hilltops and ridges yield smaller quantities of water.

Most of the water obtained from drilled wells is extremely hard and contains noticeable amounts of iron. Salty water may be found less

places.
Dug wells.-Most wells in valleys are adequate for a minimum domestic supply. A few wells dug in valleys are adequate for a modern domestic supply.

About half the wells dug on hills are adequate for a minimum domestic supply. A few wells dug on hills are adequate for a modern domestic supply.

Springs.-A few springs supply sufficient quantities of water for domestic use. Almost all springs yield less than $5 \mathrm{gpm}$

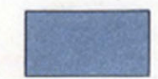

Water in the Breathitt formation (subarea 2)

Drilled wells.-More than three-quarters of the wells drilled in valley bottoms are adequate for a modern domestic supply. Nearly all wells

in valleys are adequate for a minimum domestic supply.

A bout three-quarters of the wells drilled on hillsides are adequate for a modern domestic supply. Nearly all wells on hillsides are adequate for a minimum domestic supply.

Nearly all wells drilled on hilltops are adequate for a minimum domestic supply. Some wells on hilltops or ridgetops are adequate for a modern domestic supply.

Drilled wells more than 200 feet deep in valleys may yield enough water for small municipal or industrial supplies.

Ground water obtained from most drilled wells in this area is moderately hard and contains noticeable amounts of iron. In places along the northwestern margin of the area, salty water may be found in wells tapping the Breathitt formation less Elsewhere, salty water in drilled wells probably will not be found less than 200 feet below the level of the principal valley bottoms.

Dug wells.-Most wells dug in valleys are adequate for a minimum domestic supply. A few wells dug in valleys are adequate for modern domestic supply.

Almost half the wells dug on hills are adequate for a minimum domestic supply. A few wells dug on hills are adequate for a modern

Springs. - A few springs supply sufficient quantities of water for

domestic use. Almost all springs yield less than $5 \mathrm{gpm}$

Figure 6: HLDTC (approximate location shown with a yellow circle) lies within the southern hydrologic Kanawha section (modified from Kilburn et al., 1962). 
Within Knox County, reliable quantities of groundwater are found in sand, gravel, and fractured rock. Most groundwater for domestic use is produced from relatively shallow wells $(<150 \mathrm{ft}[46 \mathrm{~m}]$ bls) drilled within fractured bedrock (shale, sandstone, siltstone, limestone or coal) or unconsolidated sediments. Joints and faults in bedrock may extend tens of feet to miles (meters to kilometers) in length. These may be identifiable as linear features on aerial photographs or satellite images. Thus far, there appears to be a direct positive correlation between length and groundwater yield (Wyrick and Borchers, 1981; Kipp and Dinger, 1991).

\subsection{Hydrology}

The HLDTC is bounded on the east, north, and west by the Cumberland River. The Cumberland River flows generally westward through Kentucky and Tennessee to its confluence with the Ohio River near Paducah, Kentucky (Figure 7). The Federal Emergency Management Authority (FEMA) Flood Insurance Rate Map for Kentucky (Figure 8) depicts most of the HLDTC as being located within the 100-year floodplain (FEMA, 2015). USGS gaging stations are located upstream at Pineville (station no. 03402900) and downstream at Barbourville (station no. 03403500) (Figure 9). River stage and discharge data are accessible from the USGS National Water Information System website (http://waterdata.usgs.gov). Several small tributaries to the Cumberland River are located throughout the study area. Perennial and ephemeral wetlands are located on the western half of the property (Figure 2). 


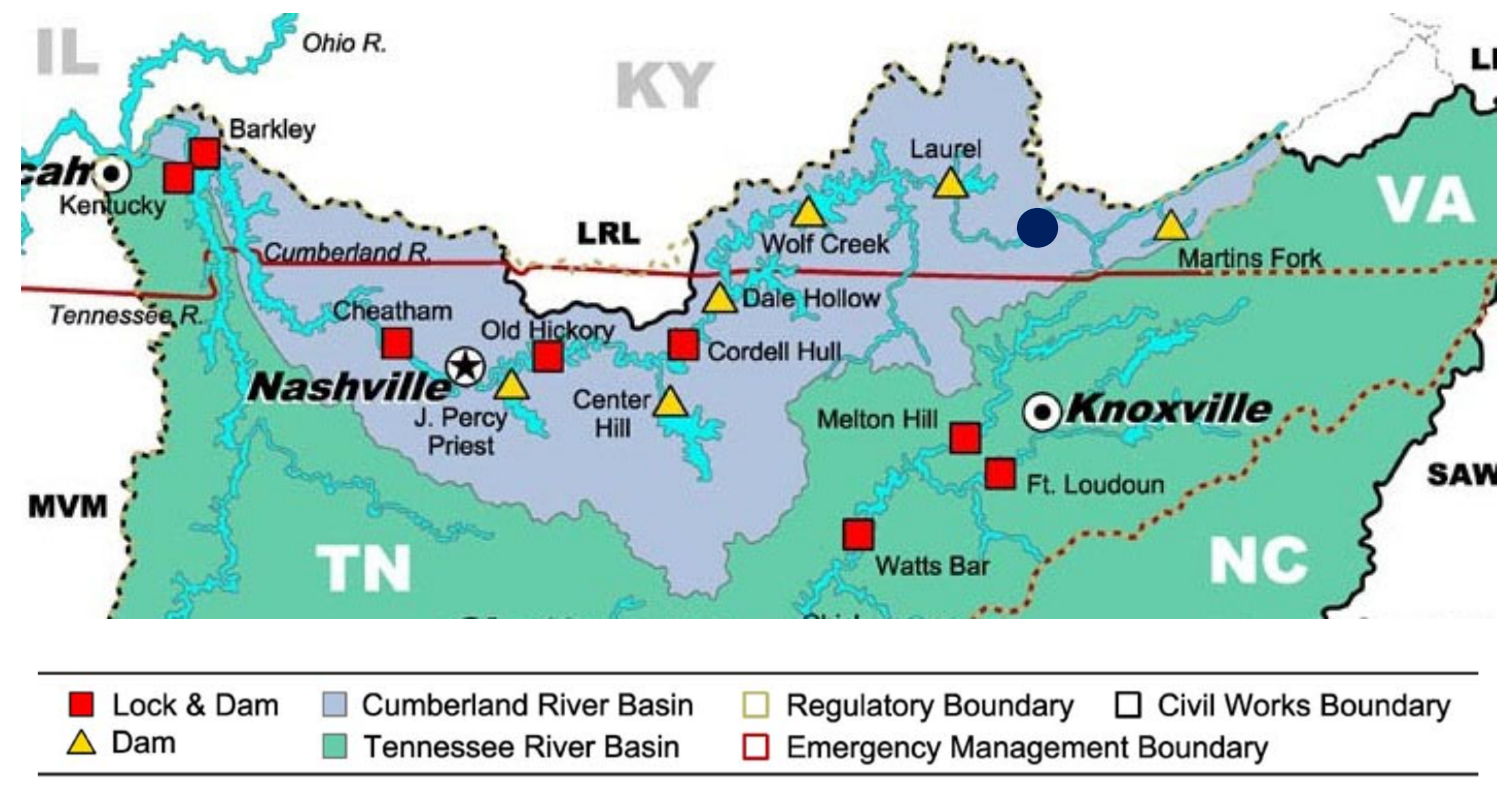

Figure 7: Cumberland River basin and approximate site location (dark blue circle) (USACE LRN, 2017). 


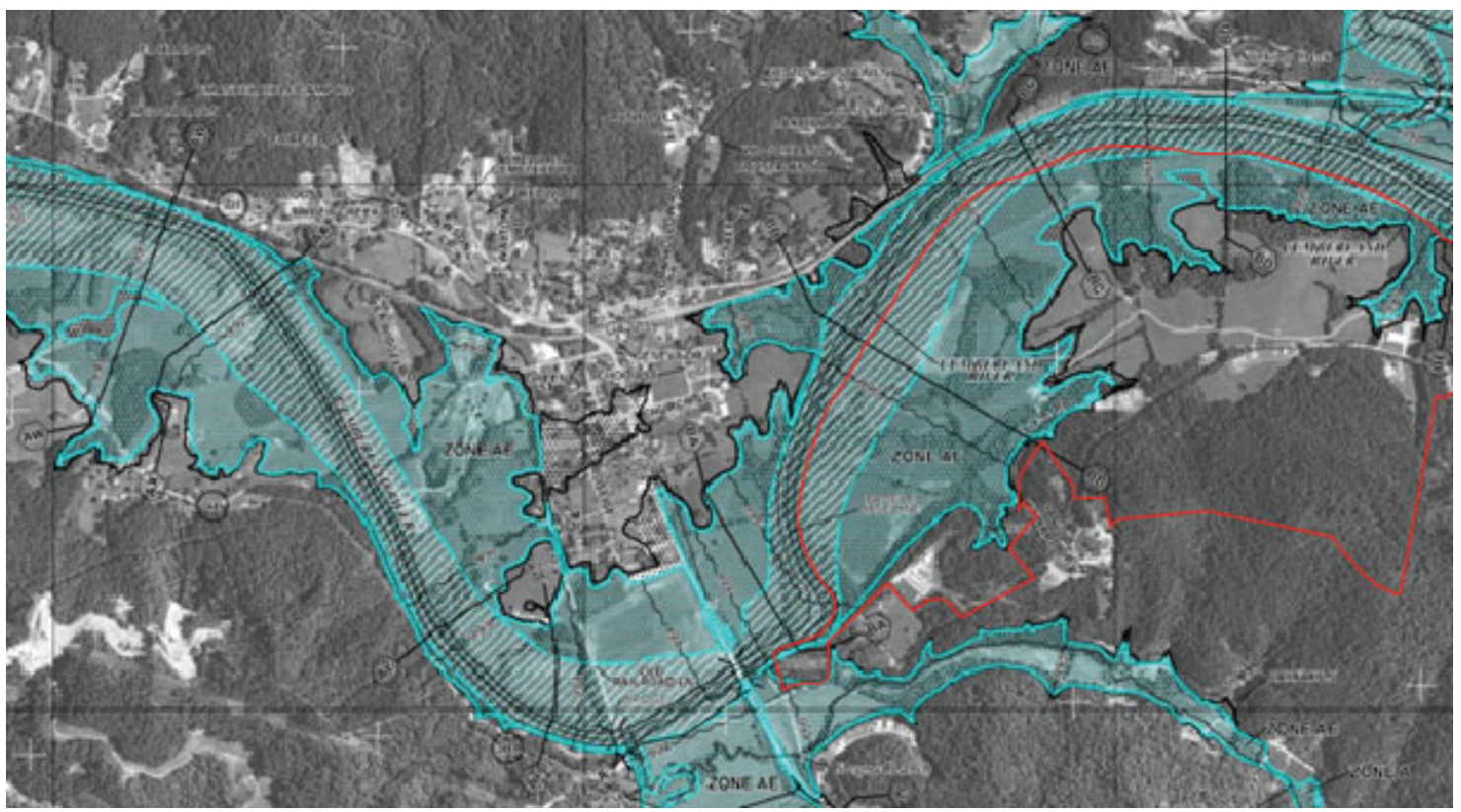

\section{LEGEND}

\section{SPECIAL FLOOD HAZARD AREAS SUBJECT TO INUNDATION BY THE $1 \%$ ANNUAL CHANCE FLOOD}

The $1 \%$ annual chance flood (100-year flood), also known as the base flood, is the flood that has a $1 \%$ chance of being equaled or exceeded in any given year. The Special Flood Hazard Area is the area subject to flooding by the $1 \%$ annual chance flood. Areas of Special Flood Hazard include Zones $A, A E, A H, A O, A R, A 99, V$, and VE. The Base Flood Elevation is the water-surface elevation of the $1 \%$ annual chance flood.

ZONE A No Base Flood Elevations determined.

ZONE AE Base Flood Elevations determined.

ZONE AH Flood depths of 1 to 3 feet (usually areas of ponding); Base Flood Elevations determined.

ZONE AO Flood depths of 1 to 3 feet (usually sheet flow on sloping terrain); average depths determined. For areas of alluvial fan flooding, velocities also determined.

ZONE AR Special Flood Hazard Area formerly protected from the $1 \%$ annual chance flood by a flood control system that was subsequently decertified. Zone AR indicates that the former flood control system is being restored to provide protection from the $1 \%$ annual chance or greater flood.

ZONE A99 Areas to be protected from $1 \%$ annual chance flood event by a Federal flood protection system under construction; no Base Flood Elevations determined.

ZONE V Coastal flood zone with velocity hazard (wave action); no Base Flood Elevations determined.

ZONE VE Coastal flood zone with velocity hazard (wave action); Base Flood Elevations determined.

\section{FLOODWAY AREAS IN ZONE AE}

The floodway is the channel of a stream plus any adjacent floodplain areas that must be kept free of encroachment so that the $1 \%$ annual chance flood can be carried without substantial increases in flood heights.

Figure 8: FEMA flood map for HLDTC (FEMA, 2015). HLDTC site boundary in red. 


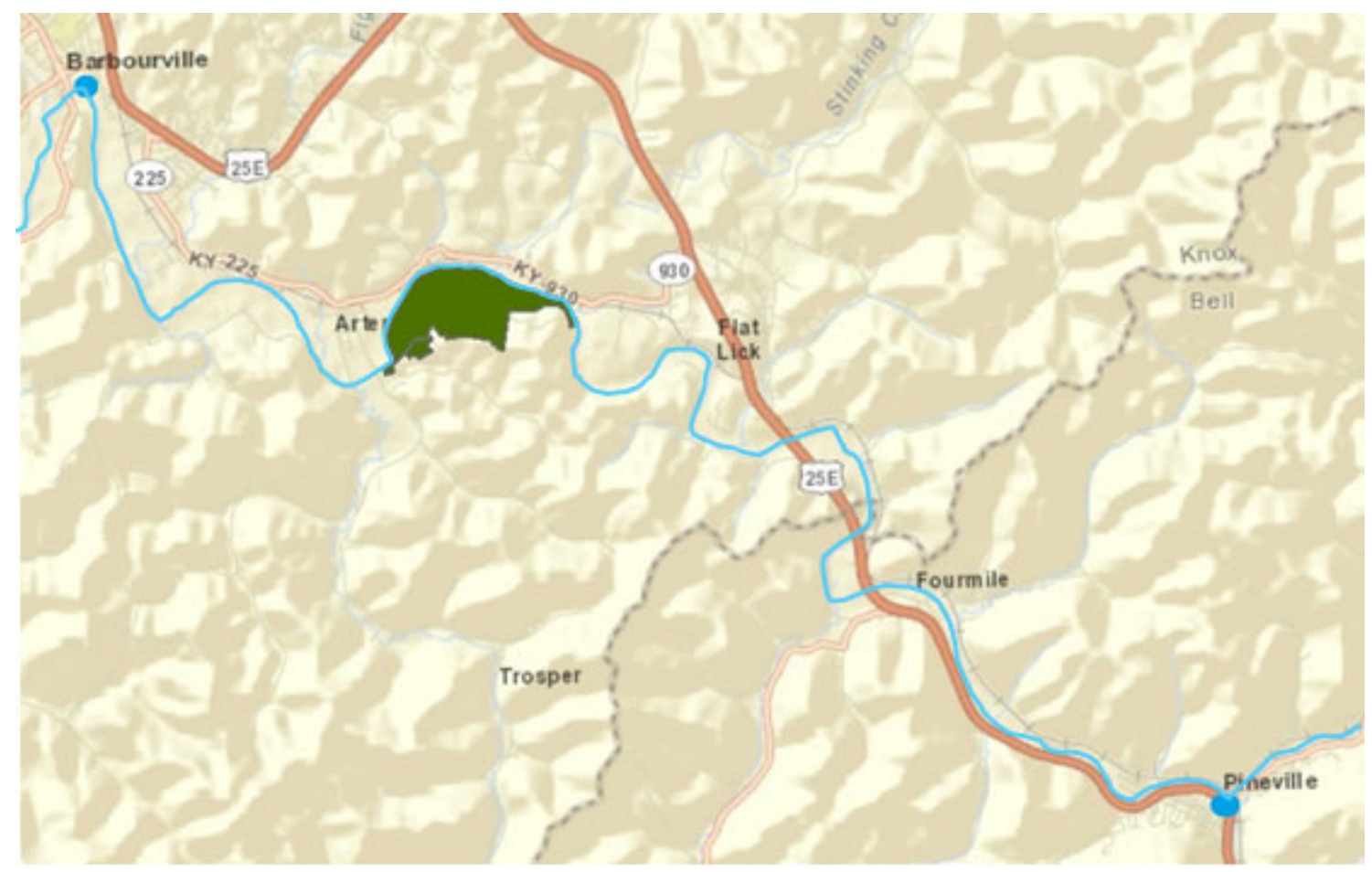

Figure 9: Approximate locations of nearest USGS gaging stations (in blue) upstream and downstream of HLDTC (in green) (modified from Sherratt, undated).

\subsection{Previous Work}

From spring 1998 to fall 2003, the Kentucky Geological Survey (KGS) conducted two consecutive water-quality assessments at HLDTC (then referred to as the Artemus Training Site) to document baseline conditions and determine representative sampling sites as part of a long-term monitoring plan. Assessments included sampling field water-quality parameters ( $\mathrm{pH}$, specific conductance $[\mathrm{SC}]$, temperature, turbidity) and solutes (metals, sulfate, nitrate, chloride, bicarbonate, and total dissolved solids [TDS]).

The assessments concluded that Artemus Training Site surface water had limited impact on the water quality of the Cumberland River. Well water had a mean $\mathrm{pH}$ of 7.64 (range 4.37-8.63) and higher concentrations of dissolved iron than onsite surface water. Other dissolved constituents, such as sulfate, bicarbonate, magnesium, calcium, and 
chloride, were considerably higher in the Cumberland River than in wells or tributaries. The impacts of resource extraction (mining, gas wells) and military activities do not appear to be detrimental to river water or off-site groundwater quality (Galceran and Dinger, 2001; Cumbie et al., 2004).

Other related work completed at the site included an integrated natural resources management plan, which provided an inventory and description of surface-water sites with an identification scheme (Snyder and Sendlein, 1997); a biological inventory (White et al., 1995); a natural resources inventory and conservation plan (USDA, 1995); and a soil survey (USDA, 1994).

\section{Methods}

\subsection{Water-Quality Assessment}

\subsubsection{Monitoring Well Installation}

Eleven monitoring wells were installed across the site in this study (Figures 10-11; Table 2). Dry holes (MW-3, MW-9, and MW-10) were re-drilled as MW-3R, MW-9R, and MW-10R, respectively. An effort was made to locate the re-drilled wells close to the original well locations. Where possible, the wells were installed in rows of two to three perpendicular to the river. The rows extend to the base of the ridge along the southern boundary. Proposed well locations were coordinated with and approved by appropriate KNG personnel and utility clearances were obtained prior to installation. 


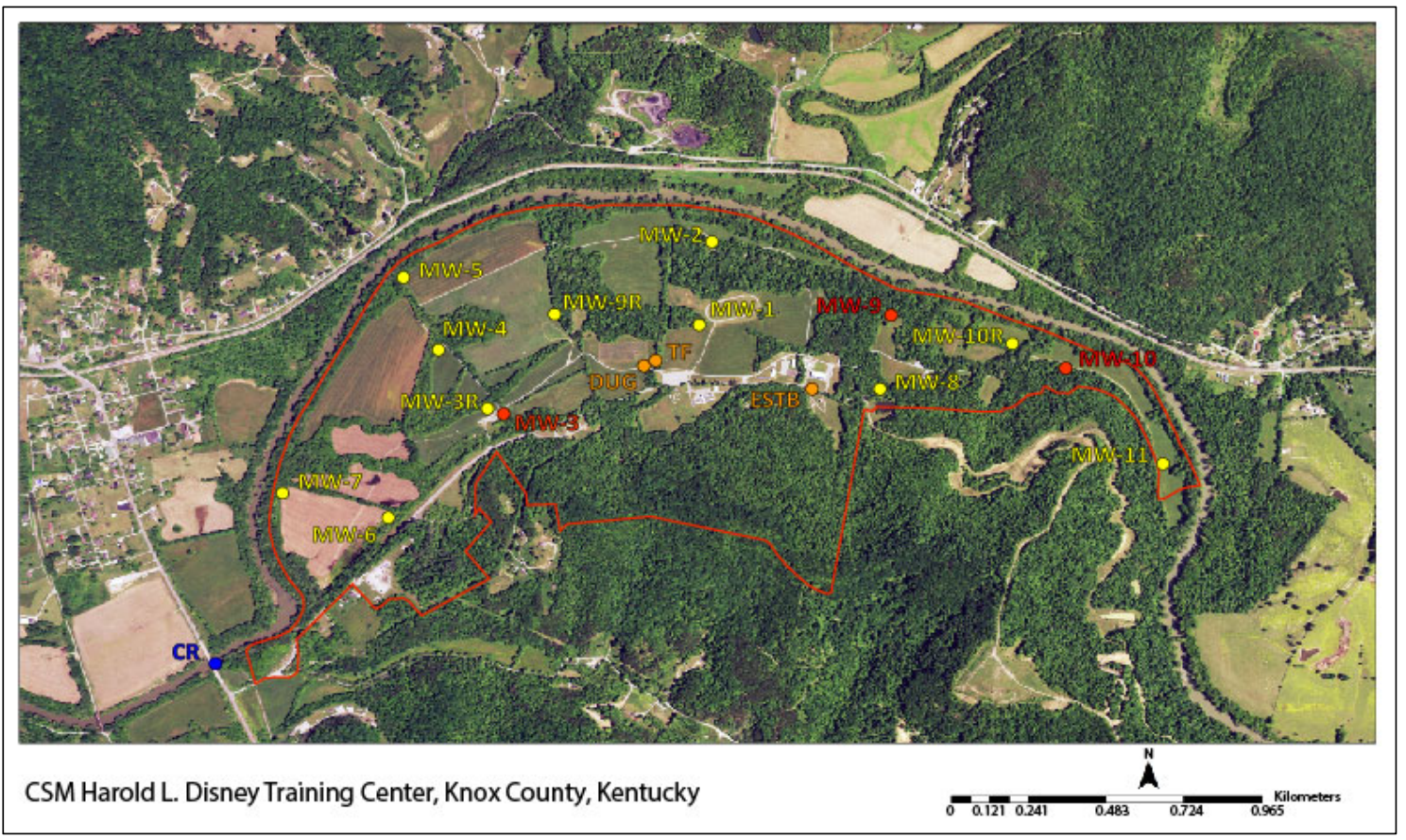

Figure 10: Location of monitoring wells (shown as yellow dots), dry well locations (red dots), existing wells (orange dots), and Cumberland River sample point (blue dot).

Geo-Drill, Inc. was contracted to install the monitoring wells in December 2016 by direct push to refusal, followed by drilling with hollow-stem auger. Cores were collected at 1.5-m intervals. Well construction consisted of drilling $1.5 \mathrm{~m}$ into bedrock (where possible), $1.5 \mathrm{~m}$ of 2 -in. $(5.1-\mathrm{cm})$ nominal PVC screen with pea gravel rising $0.2 \mathrm{~m}$ above the screen, then solid 2-in. $(5.1-\mathrm{cm})$ nominal PVC casing and bentonite to land surface, and finished with a $2-\mathrm{ft} \times 2-\mathrm{ft}(0.6-\mathrm{m} \times 0.6-\mathrm{m})$ concrete pad and locking steel riser having a 0.9-m casing stickup. Well depths ranged from 4.3 to $13.6 \mathrm{~m}$. The Uniform Kentucky Well Construction Records are included in the appendices. 


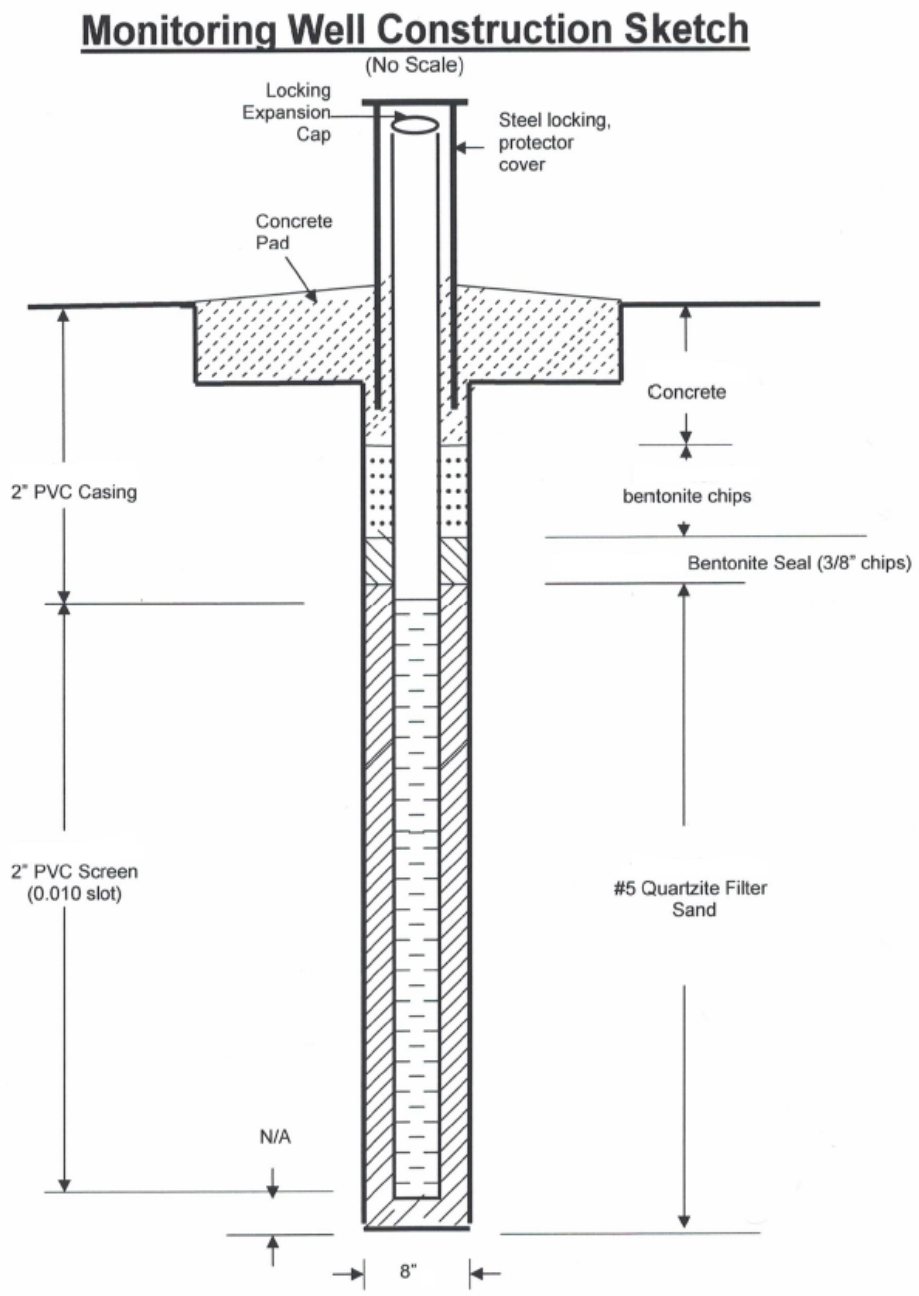

Figure 11: Generalized monitoring well construction sketch for HLDTC.

After installation, Geo-Drill, Inc. developed the wells (Well Development Record) until the produced water was no longer turbid. All monitoring well locations were surveyed for latitude, longitude, and elevations by Donovan Sherratt (KNG) in July 2017 (Table 2). 
Table 2. HLDTC Well Locations and Cumberland River Sample Site

\begin{tabular}{|c|c|c|c|c|}
\hline Sample Sites & Latitude (dms) & $\begin{array}{l}\text { Longitude } \\
\text { (dms) }\end{array}$ & $\begin{array}{l}\text { Elevation } \\
\text { (m asl) }\end{array}$ & $\begin{array}{l}\text { Depth } \\
\text { (m bls) }\end{array}$ \\
\hline MW-1 & $36^{\circ} 50^{\prime} 3.689^{\prime \prime}$ & $-83^{\circ} 49^{\prime} 7.432^{\prime \prime}$ & 311.5 & 5.5 \\
\hline MW-2 & $36^{\circ} 50^{\prime} 12.739^{\prime \prime}$ & $-83^{\circ} 49^{\prime} 05.600^{\prime \prime}$ & 281 & 8.8 \\
\hline MW-3R & $36^{\circ} 49^{\prime} 55.067^{\prime \prime}$ & $-83^{\circ} 49^{\prime} 34.561^{\prime \prime}$ & 301.4 & 6.9 \\
\hline MW-4 & $36^{\circ} 50^{\prime} 1.579^{\prime \prime}$ & $-83^{\circ} 49^{\prime} 40.609^{\prime \prime}$ & 301.1 & 9.1 \\
\hline MW-5 & $36^{\circ} 50^{\prime} 9.492^{\prime \prime}$ & $-83^{\circ} 49^{\prime} 45.350^{\prime \prime}$ & 301.4 & 13.6 \\
\hline MW-6 & $36^{\circ} 49^{\prime} 43.504^{\prime \prime}$ & $-83^{\circ} 49^{\prime} 47.330^{\prime \prime}$ & 299.3 & 11.9 \\
\hline MW-7 & $36^{\circ} 49^{\prime} 46.405^{\prime \prime}$ & $-83^{\circ} 50^{\prime} 0.956^{\prime \prime}$ & 300.2 & 12.6 \\
\hline MW-8 & $36^{\circ} 49^{\prime} 56.450^{\prime \prime}$ & $-83^{\circ} 48^{\prime} 44.600^{\prime \prime}$ & 308.5 & 4.3 \\
\hline MW-9R & $36^{\circ} 50^{\prime} 5.122^{\prime \prime}$ & $-83^{\circ} 49^{\prime} 25.882^{\prime \prime}$ & 305.1 & 6.9 \\
\hline MW-10R & $36^{\circ} 50^{\prime} 1.115^{\prime}$, & $-83^{\circ} 48^{\prime} 27.515^{\prime \prime}$ & 301.1 & 9.1 \\
\hline MW-11 & $36^{\circ} 49^{\prime} 47.629^{\prime \prime}$ & $-83^{\circ} 48^{\prime} 8.636^{\prime \prime}$ & 302.1 & 8.1 \\
\hline Tree Farm (TF) & $36^{\circ} 50^{\prime} 0.107^{\prime}$ & $-83^{\circ} 49^{\prime} 13.523^{\prime \prime}$ & 314.2 & 36.6 \\
\hline $\begin{array}{l}\text { Cumberland River } \\
\text { (CR) }\end{array}$ & $36^{\circ} 49^{\prime} 28.0^{\prime \prime}$ & $-83^{\circ} 50^{\prime} 10.0^{\prime \prime}$ & -- & -- \\
\hline
\end{tabular}

Notes:

-- No data

\subsubsection{Water Sampling}

The installed monitoring wells, an on-site tree-farm well, and the Cumberland River were sampled quarterly starting January 2017 and concluding January 2018 (January 2017, April 2017, July 2017, October 2017, January 2018). The tree-farm well was drilled to 120 $\mathrm{ft}(36.6 \mathrm{~m})$ bls, with the top of bedrock encountered at $27 \mathrm{ft}(8.2 \mathrm{~m})$ bls. That well was cased to a depth of $38 \mathrm{ft}(12 \mathrm{~m})$ bls, below which the hole was open. The monitoring wells were sampled with a low-flow peristaltic pump within the well-casing screen interval and with dedicated tubing, while the tree-farm well was sampled from within the open borehole using a submersible ("trash") pump with dedicated tubing. During well purging, the waterquality parameters (temperature, $\mathrm{SC}$, dissolved oxygen [DO], $\mathrm{pH}, \mathrm{Eh}$ ) were monitored with sensors every 5 min until the parameters attained stability (defined as three consecutive readings within the instrument's sensor accuracy, when possible). Thereafter, water 
samples were collected through 0.45 -micron in-line filters (for DO [by titration], alkalinity, anions, metals, nutrients, dissolved organic carbon [DOC], volatile organic compounds [VOCs], isotopes of water $\left[\delta^{18} \mathrm{O}\right.$ and $\left.\left.\delta^{2} \mathrm{H}\right]\right)$, preserved as needed, then refrigerated and analyzed within specified holding times or frozen prior to analyses (for nutrients). The following laboratories and methods were selected for conducting the analyses:

- KGS: anions (by ion chromatography), metals (by inductively coupled plasma optical-emission spectrometry), nutrients, DOC (referred to as TOC in lab reports)

- McCoy and McCoy Laboratories: VOCs (USEPA method 8260B), collected in April and October 2017

- University of Wyoming, Stable Isotope Facility: isotopes (cavity ring-down spectroscopy).

- $\mathrm{HACH}$ digital titrator test kits for DO and alkalinity, model AL-DT: azide modification of Winkler method (method 8215) and total alkalinity (method 8230), respectively.

The Cumberland River sample was collected with a bailer from a bridge; therefore, monitoring water quality parameters and stability attainment were not applicable. Water samples were collected for the same analyses as the wells. Mean values for data were calculated and reported in accordance with EPA Guidance for Data Quality Assessment (2000) and MDL values were handled in accordance with EPA Regional Guidance on Handling Chemical Concentration Data Near the Detection Limit on Risk Assessments (1991).

Solute chemistry was assessed using Geochemist's Workbench (student edition) to generate Piper diagrams and PHREEQC (Version 3) (Parkhurst and Appelo, 2013) for 
calculating charge-balance errors (CBE\%) and determining saturation index (SI) values. Input parameters for the Piper diagrams included $\mathrm{pH}$ (Table 3), major cations $\left(\mathrm{Ca}^{2+}, \mathrm{Mg}^{2+}\right.$, $\left.\mathrm{Mn}^{2+}, \mathrm{K}^{+}, \mathrm{Na}^{+}\right)$(Table 4), minor cation $\left(\mathrm{Fe}^{2+}\right)$ (Table 5), anions and nutrients $\left(\mathrm{Cl}^{-}, \mathrm{HCO}_{3}^{-}\right.$, $\mathrm{SO}_{4}{ }^{2-}, \mathrm{NO}_{3}{ }^{-}$) (Table 7). For PHREEQC, parameter inputs included temperature, $\mathrm{pH}$ (Table 3), pe (which was set to the program default), cations (Table 4 and 5), anions and nutrients (Table 7). For analytes below the laboratory method detection limit (MDL), concentrations are assumed to be zero. Saturation index values were calculated for mineral phases likely present within aquifer sediments, such as carbonates (calcite, dolomite, siderite, strontianite, witherite), sulfates (anhydrite, barite, celestite, gypsum, melanterite), iron (oxyhydr)oxides (goethite, hematite, melanterite, amorphous $\left.\mathrm{Fe}(\mathrm{OH})_{3}\right)$, manganese (oxyhydr)oxides (hausmannite, manganite, pyrochroite, pyrolusite), silica $\left(\mathrm{SiO}_{2}\right)$ phases, fluorite, and halite. Saturation index values were not calculated for aluminosilicate minerals and pyrite, as aluminum $\left(\mathrm{Al}^{3+}\right)$ and sulfide $\left(\mathrm{S}^{2-}\right)$ were close to or below the MDL, and $\mathrm{Al}$ is assumed to be conserved in the solid phase (Haile and Fryar, 2017).

\subsection{Site Lithology}

\subsubsection{Soil Core Logs}

Subsurface lithology was determined by examining the 2-in. $(5.1-\mathrm{cm})$ cores collected while installing monitoring wells. During the direct-push drilling, the tops and bottoms of cores were field-logged for texture and color using a Munsell soil chart. Upon completion of the wells, the cores were brought back to the University of Kentucky hydrogeology lab for detailed logging. This consisted of splitting the cores in half, where one half of the core was preserved and the other half was photographed and evaluated for texture, structures, and color using a Munsell soil chart. Hand-drawn soil core logs were 
generated and later converted to digital core logs with SedLog (open-source software) and Adobe Illustrator. The preserved core half was later used for analyses of grain size, carbon content, and bulk chemistry, as detailed below.

\subsubsection{Grain Size Analysis}

The grain size was determined for a subset of cores by hydrometer and sieve analysis. Core sections with sandier fractions, as determined during soil core characterizations, were analyzed following Catto and Quaternary Research Group (1989). Analyses were performed for a total of 16 coarser-grained core sections from the west half of the site, including seven of the 11 monitoring wells (MW-1, MW-3R, MW-4, MW-5, MW-7, MW-8, MW-9R).

Hydrometer analysis was used to determine proportions of silt and clay by applying Stokes' Law. It states that a spherical particle settling at a uniform velocity will encounter a resisting force due to the properties of the liquid. By determining the settling velocity, the particle radius can be calculated. To determine this, the selected soil core samples were disaggregated, passed through a \#10 (2-mm) mesh sieve, and dispersed overnight in a 4\% sodium hexametaphosphate solution. Thereafter, the soil solution was agitated and transferred to a graduated cylinder. Hydrometer readings were taken over a period of $24 \mathrm{hr}$ (15 and $30 \mathrm{~s} ; 1,2,10,15,30,60$, and $120 \mathrm{~min} ; 4,8$, and $24 \mathrm{hr}$ ) in both the sample and a control cylinder. The control cylinder was used to correct the hydrometer readings for density differences due to temperature fluctuations.

After hydrometer analysis, sieve analysis was performed to determine proportions of sand- to granule-sized grains. The soil solution within the sample cylinder was poured through a \#230 (0.063-mm) sieve, thoroughly rinsed, and dried in the oven overnight at 50 
${ }^{\circ} \mathrm{C}$. The dried soil sample was placed on a shaker for $15 \mathrm{~min}$ and passed through a stack of sieves (numbers $35[0.5 \mathrm{~mm}], 60$ [0.25 mm], $120[0.125 \mathrm{~mm}], 140[0.105 \mathrm{~mm}], 170[0.088$ $\mathrm{mm}]$, and $230[0.0625 \mathrm{~mm}])$. The soil mass retained on each sieve was weighed. The percentages of sand, silt, and clay were determined for analyzed core sections by combining the hydrometer and sieve analyses results.

\subsubsection{Carbon Content}

In total, 44 samples were collected from soil cores (MW-1 cores 1-3; MW-2 cores 1, 2, 5; MW-3R cores 1-4; MW-4 cores 1, 2, 5; MW-5 cores 1-4; MW-6 1, 3, 5; MW-7 cores 1, 2, 4, 5; MW-8 cores 1-2; MW-9R cores 1-4; MW-10R cores 1, 2, 4; MW-11 cores $1,2,5)$ at points of visible color or texture transition. The samples were analyzed for total carbon (TC) and total inorganic carbon (TIC). Total organic carbon was calculated by taking the difference between TC and TIC values.

TC samples were combusted using a LECO model SC-144DR instrument, which was calibrated using known carbon standards ( $\mathrm{TC} \% 0.53 \pm 0.03,0.98 \pm 0.03,4.98 \pm 0.05$, $6.25 \pm 0.21)$. Approximately $0.3 \mathrm{~g}$ of soil was weighed into a ceramic crucible and mixed with COM-CAT, a combustible accelerator, and then placed into the LECO furnace for combustion. The instrument program SC-144DR Sulfur/Carbon Determinator was used to determine the sample TC values using the low-carbon method.

TIC analyses were performed using a carbon coulometer (UIC Coulometrics Inc., model CM5130) with acidification module (model CM5130). It was calibrated using a known inorganic carbon standard $\left(\mathrm{CaCO}_{3}\right)$ with a mass between $10.5-11.5 \mathrm{mg}$, which results in a TIC range of $11.7-12.2 \%$ carbon. Approximately $45 \mathrm{mg}$ of soil were placed into the acidification module containing a 3\% silver nitrate acidified with sulfuric acid to 
$\mathrm{pH}$ 3. The acidified sample solution was then internally transferred to the coulometer, which determined the sample TIC values.

Soil analytical data were calculated and reported in accordance with EPA Guidance for Data Quality Assessment (2000) and MDL values were handled in accordance with EPA Regional Guidance on Handling Chemical Concentration Data Near the Detection Limit on Risk Assessments (1991).

\subsubsection{Mineral Composition}

The 44 samples collected from the soil cores for carbon analyses were also used to determine the soil elemental composition via X-ray fluorescence (XRF). Sample pellets were formed using a Carver laboratory press and die-set to create $\sim 4$-cm soil pellets surrounded by a 3-4 mm-thick boric acid barrier. Prior to analysis, the formed pellets were dried overnight. Analysis of major elements (Na, Mg, Al, Si, P, S, K, Ca, Ba, Ti, V, Cr, Mn, Fe, Co, Ni, $\mathrm{Cu}, \mathrm{Zn}$ ) and minor elements (As, Pb, Th, Rb, U, Sr, Y, Zr, Nb, Mo, Rh, Sn, Sb) utilized a Bruker model Tracer IV-SD calibrated with the USGS standard SARM41 and analyzed with the S1PXRF program.

\subsection{Site Hydrogeology}

\subsubsection{Hydraulic-Head Monitoring}

The water level in each well was measured and recorded quarterly prior to sampling with a graduated tape measure (e-line). Additionally, Solinst Levelogger Jrs. were installed in MW-6 and MW-7 to record hourly water levels and temperatures. A Barologger was installed in MW-6 to record air temperature and barometric pressure, which was used to correct the recorded water levels to hydraulic heads. Water-table maps were generated quarterly using the aforementioned data. 


\subsubsection{Cumberland River Hydrographs and Precipitation}

Hydrographs were created using the Cumberland River stage data from the Barbourville and Pineville USGS gages and continuous hydraulic-head data for wells MW6 and MW-7. The Barbourville gage is located $\sim 10 \mathrm{~km}$ downstream of HLDTC and the Pineville gage is $\sim 21 \mathrm{~km}$ upstream. River-stage response to precipitation events was assessed by plotting the gage precipitation data onto the hydrographs. Stage and precipitation were recorded at 30-min intervals at both gages.

\subsubsection{Hydraulic Conductivity}

Hydraulic conductivity was evaluated by grain-size analyses and hydraulic testing. The grain-size values determined for the subset of cores by hydrometer and sieve analysis were input into HydrogeoSieveXL, an open-source Excel program for estimating hydraulic conductivity based on empirical relationships with grain size (Devlin, 2015).

In March 2018, aquifer tests were conducted by pumping wells MW-5, MW-6, and MW-7 and by slugging the remaining monitoring wells. The data for MW-3R and MW-9R were inadvertently overwritten and thus were not available for analysis. Before beginning the slug and pump testing, the monitoring well plugs were loosened and wells were allowed to equilibrate with atmospheric pressure overnight.

To initiate slug testing, a Schlumberger Diver was placed into the well at a recorded depth near the bottom of the well and below the slug. Initial water level was recorded with an e-line, then a $1.5-\mathrm{m}$ section of 1-in. $(2.54-\mathrm{cm})$ nominal diameter PVC filled with sand and suspended from a line was quickly lowered into the monitoring well and hung above the Diver. Water-level response was recorded for a period of $\sim 7 \mathrm{hr}$. Thereafter, a final water level was measured by e-line prior to retrieval of the slug and Diver. 
Single well pumping tests were conducted at HLDTC. To initiate pumping tests, a Solinst Levelogger or Schlumberger Diver was placed into the well at a recorded depth within the well screen. A submersible pump (Proactive, Mini-Monsoon) fitted with dedicated well tubing was then lowered into the well and hung above the Levelogger/Diver. At land surface, the pump was attached to a custom-made flow-rate board. An initial water level was taken by e-line and the pump was then turned on. Water-level drawdown and recovery were recorded on the Levelogger/Diver as well as taken by e-line. A final water level was recorded by e-line prior to removal of pump and Levelogger/Diver. During testing, the flow-rate board allowed for adjustments to maintain a relatively steady pumping rate. As MW-6 and MW-7 were likely overtopped during flooding in midFebruary 2018, water samples were collected during the pumping tests for analyses of chloride and stable isotopes to determine what, if any, impact the floodwaters had on the monitoring wells. Results of both slug and pumping tests were analyzed for measurement of hydraulic conductivity using AQTESOLV software.

\subsection{Monitoring Well Abandonment}

CSI Drilling, LLC (formerly Geo-Drill, Inc.) abandoned nine of the 11 installed monitoring wells (all except MW-3R and MW-6) in June 2018. Wells were abandoned by removing the pad and protective riser, then overdrilling the well to remove the casing, screen, grout and filter pack; grouting the borehole from the bottom up with bentonite chips to $0.6 \mathrm{~m}$ bls; and finishing with native backfill to land surface. The Uniform Kentucky Well Maintenance and Plugging Records are included in the appendices. 


\section{Results and Discussion}

\subsection{Water-Quality Assessment}

Water samples were collected from January 2017 to March 2018. During this time the mean air temperature was $15.8^{\circ} \mathrm{C}$ (range $-11.5^{\circ} \mathrm{C}$ in January 2018 to $33.9^{\circ} \mathrm{C}$ in July 2017), mean groundwater temperature was $13.9^{\circ} \mathrm{C}$ (range $11.1-19.3^{\circ} \mathrm{C}$ ), and mean surface water temperature in the Cumberland River was $11.0{ }^{\circ} \mathrm{C}$ (range $0.7^{\circ} \mathrm{C}$ in January 2018 to $24.9{ }^{\circ} \mathrm{C}$ in July 2017). Figure 12 depicts the air temperature (24-hr moving average) with groundwater temperatures in MW-6 and MW-7, as recorded by Solinst loggers. Both monitoring wells documented the flood event that occurred in February 2018. During that flood, inundation of monitoring wells MW-6 and MW-7 was indicated by a sharp watertemperature decline of several degrees Celsius. According to the Barbourville Cumberland River gage, this was the highest stage since March 19, 2002. The event was the ninth largest since the period of continuous record began in 1949, yielding a recurrence interval of 7.1 years. The monitoring wells also depict a seasonal groundwater temperature cycle where the high occurs around November/December and the low in April/May. These highs and lows lag $\sim 4$ months behind the air temperature high in July and low in January. Generally, groundwater temperatures are considered to be fairly stable due to low thermal conductivity of rocks and soil and reflect mean ground surface temperature. However, more pronounced seasonal temperature variations are observed in shallow groundwater $(<$ $10 \mathrm{~m}$ bls) or in areas with high thermal and hydraulic conductivity (Ohtani et al., 2015). 


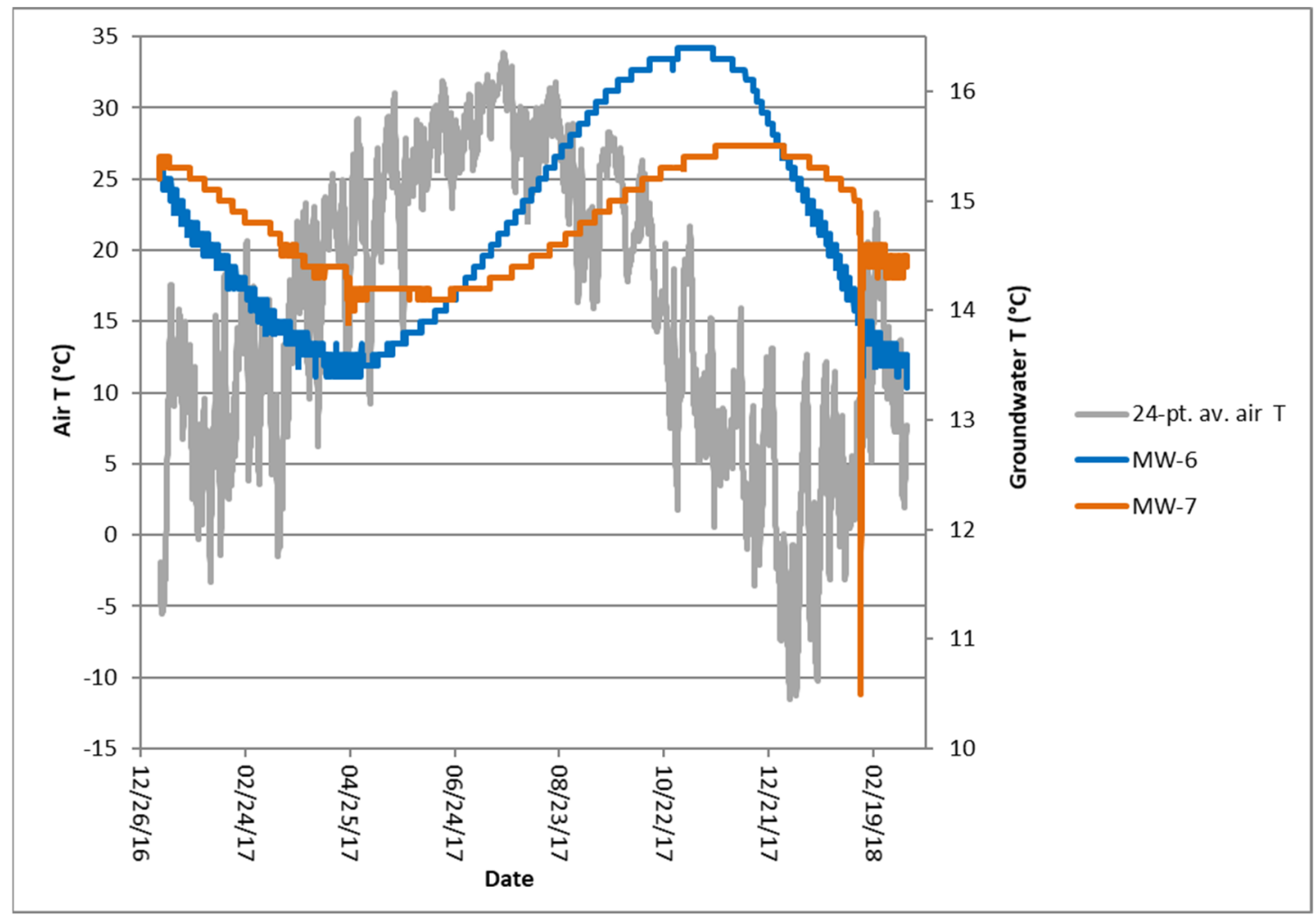

Figure 12: Air (24-point moving average) and groundwater temperatures at HLDTC from January 2017 to March 2018.

The collected quarterly water-quality parameters (Table 3) characterize the groundwater as acidic, dysoxic $(0.2-2.0 \mathrm{mg} / \mathrm{L})$ to slightly oxic $(2.0-8.0 \mathrm{mg} / \mathrm{L})$, and relatively dilute. Mean $\mathrm{pH}$ was 5.90 (range 4.12-6.76) and mean TDS was $82 \mathrm{mg} / \mathrm{L}$ (range 11-321 mg/L). Total dissolved solids (TDS) were estimated from SC using a conductivity converter (https://www.lenntech.com/calculators/conductivity/tds_engels.htm). Mean specific conductance (SC) was $114.2 \mu \mathrm{S} / \mathrm{cm}(17-502 \mu \mathrm{S} / \mathrm{cm})$. MW-2 exhibited unusually high SC values (103-502 $\mu \mathrm{S} / \mathrm{cm}$, mean $256.8 \mu \mathrm{S} / \mathrm{cm})$ and steadily decreased across sampling events. During sampling, MW-2 was observed as being very turbid and stability parameters were difficult to obtain prior to sampling. It is believed that MW-2 may not have been properly developed. Dissolved oxygen averaged $2.03 \mathrm{mg} / \mathrm{L}$ (range $0.23-8.47$ $\mathrm{mg} / \mathrm{L}$ ) via YSI electrode measurement and $2.83 \mathrm{mg} / \mathrm{L}$ (range $0.18-6.56 \mathrm{mg} / \mathrm{L}$ ) via Winkler 
titration. In general, DO values for groundwater were lower for electrode measurements than for Winkler titration, which is consistent with the lack of aeration of the electrodes in the flow cell. Compared to the well data, surface water in the Cumberland River was neutral to slightly basic, oxic (range $2.0-8.0 \mathrm{mg} / \mathrm{L}$ ), and still relatively dilute. Mean $\mathrm{pH}$ was 8.35 (range 7.62-9.26) and mean TDS was $218 \mathrm{mg} / \mathrm{L}(126-289 \mathrm{mg} / \mathrm{L})$. Dissolved oxygen averaged $10.4 \mathrm{mg} / \mathrm{L}$ (range $7.32-13.45 \mathrm{mg} / \mathrm{L}$ ) by YSI and $9.09 \mathrm{mg} / \mathrm{L}$ (range $6.90-11.78$ $\mathrm{mg} / \mathrm{L}$ ) by Winkler; a flow cell was not used for river sampling. Values for $\mathrm{pH}$, TDS and DO in the Cumberland River were greater than the onsite wells.

At the site scale, wells closer to the ridge (MW-1, MW-3R, MW-6, MW-8) exhibited more stable temperatures than wells near the river (MW-5, MW-7, MW-10R). The latter wells were colder in the winter and warmer in the summer. Wells closer to the river exhibited a slightly higher $\mathrm{pH}$. The wells located in the eastern portion of the site (MW-10R, MW-11) exhibited lower pH levels, slightly lower temperatures, and higher DO than those in the west (MW-4, MW-5, MW-6, MW-7). The pH values for both groundwater and surface water are outside of the National Secondary Drinking Water Regulations (NSDWRs) set for drinking water, $\mathrm{pH} 6.5-8.5$, and should be considered when evaluating either as a drinking-water source. 
Table 3. HLDTC Water-Quality Parameters

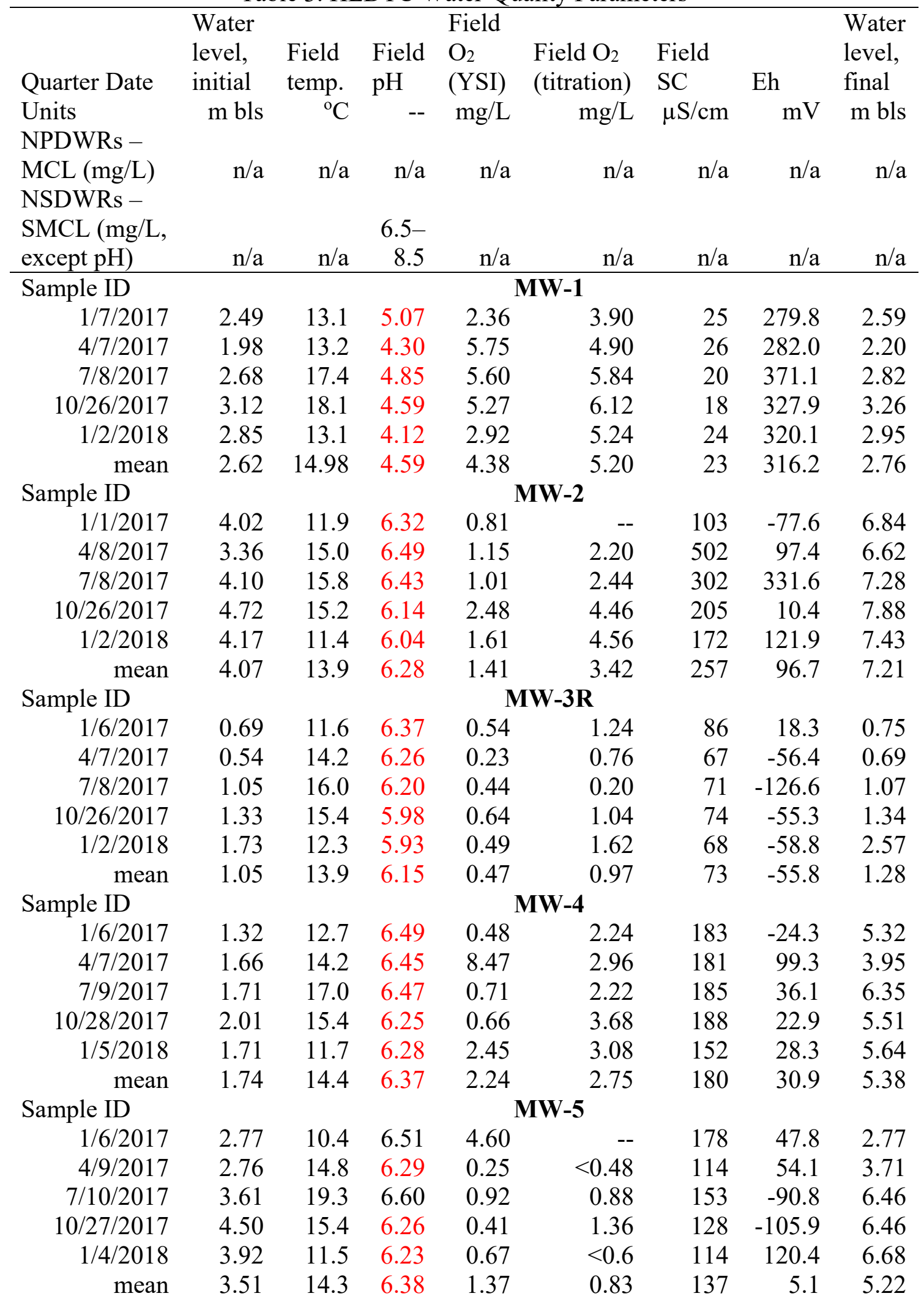


Table 3. HLDTC Water-Quality Parameters (cont.)

\begin{tabular}{|c|c|c|c|c|c|c|c|c|}
\hline Sample ID & \multicolumn{8}{|c|}{ MW-6 } \\
\hline $1 / 5 / 2017$ & 1.58 & 13.0 & 6.66 & 0.45 & $<2$ & 228 & 119.5 & 1.64 \\
\hline $4 / 9 / 2017$ & 1.81 & 15.2 & 6.62 & 0.35 & $<0.48$ & 186 & -- & 1.82 \\
\hline $7 / 10 / 2017$ & 2.05 & 15.4 & 6.57 & 0.61 & $<0.6$ & 187 & -99.2 & 2.10 \\
\hline $10 / 27 / 2017$ & 2.33 & 15.0 & 6.39 & 0.47 & $<0.6$ & 195 & -107.0 & 2.39 \\
\hline $1 / 5 / 2018$ & 1.83 & 12.6 & 6.40 & 0.71 & 0.60 & 182 & -105.4 & 1.88 \\
\hline mean & 1.89 & 14.1 & 6.52 & 0.52 & 0.86 & 193 & -104.3 & 1.93 \\
\hline Sample ID & \multicolumn{8}{|c|}{ MW-7 } \\
\hline $1 / 5 / 2017$ & 5.12 & 13.2 & 6.73 & 0.30 & 0.18 & 165 & 72.3 & 6.56 \\
\hline $4 / 9 / 2017$ & 4.99 & 15.8 & 6.76 & 0.31 & $<0.48$ & 146 & -- & 5.96 \\
\hline $7 / 10 / 2017$ & 5.34 & 16.4 & 6.67 & 0.47 & $<0.6$ & 145 & -115.3 & 6.41 \\
\hline $10 / 27 / 2017$ & 5.76 & 14.2 & 6.47 & 0.44 & $<0.6$ & 144 & -98.3 & 6.81 \\
\hline $1 / 5 / 2018$ & 5.61 & 12.6 & 6.49 & 0.85 & 0.40 & 142 & -120.6 & 6.44 \\
\hline mean & 5.36 & 14.8 & 6.63 & 0.47 & 0.45 & 148 & -112.4 & 6.43 \\
\hline Sample ID & \multicolumn{8}{|c|}{ MW-8 } \\
\hline $1 / 7 / 2017$ & 0.83 & 11.1 & 4.97 & 1.74 & 2.48 & 25 & 209.1 & 1.51 \\
\hline $4 / 8 / 2017$ & 0.83 & 11.5 & 4.97 & 0.74 & 2.34 & 22 & 228.5 & 2.74 \\
\hline $7 / 9 / 2017$ & 1.05 & 17.1 & 5.21 & 0.66 & 2.72 & 27 & 233.3 & 2.89 \\
\hline $10 / 27 / 2017$ & 1.11 & 17.6 & 5.11 & 0.68 & 2.68 & 33 & 209.6 & 2.89 \\
\hline $1 / 3 / 2018$ & 0.95 & 12.9 & 4.72 & 2.93 & 4.34 & 28 & 227.1 & 4.27 \\
\hline mean & 0.95 & 14.0 & 5.00 & 1.35 & 2.91 & 27 & 221.5 & 2.86 \\
\hline Sample ID & \multicolumn{8}{|c|}{ MW-9R } \\
\hline $1 / 7 / 2017$ & 2.37 & 11.8 & 6.64 & 1.94 & 2.50 & 202 & -16.6 & 5.82 \\
\hline $4 / 9 / 2017$ & 1.97 & 12.8 & 6.40 & 1.12 & 4.56 & 114 & 121.4 & 4.77 \\
\hline $7 / 8 / 2017$ & 2.51 & 15.3 & 6.54 & 1.37 & 3.64 & 120 & 31.4 & 5.88 \\
\hline $10 / 26 / 2017$ & 3.17 & 15.9 & 6.20 & 1.26 & 2.26 & 117 & 49.0 & 5.88 \\
\hline $1 / 4 / 2018$ & 2.77 & 12.2 & 5.88 & 2.75 & 4.34 & 78 & 63.6 & 5.37 \\
\hline mean & 2.56 & 13.6 & 6.33 & 1.69 & 3.46 & 126 & 49.8 & 5.54 \\
\hline Sample ID & \multicolumn{8}{|c|}{ MW-10R } \\
\hline $1 / 8 / 2017$ & 5.76 & 11.4 & 5.43 & 2.33 & 3.28 & 21 & -205.8 & 6.12 \\
\hline $4 / 8 / 2017$ & 4.38 & 14.0 & 5.16 & 4.09 & 4.80 & 17 & 209.8 & 4.84 \\
\hline $7 / 9 / 2017$ & 5.27 & 15.1 & 5.16 & 5.44 & 5.74 & 17 & 238.3 & 5.66 \\
\hline $10 / 27 / 2017$ & 5.01 & 15.0 & 4.94 & 4.63 & 5.68 & 20 & 173.6 & 6.60 \\
\hline $1 / 1 / 2018$ & 2.77 & 11.9 & 4.76 & 3.27 & 5.28 & 18 & 206.9 & 5.37 \\
\hline mean & 4.83 & 13.1 & 5.15 & 3.68 & 4.68 & 19 & 69.5 & 5.79 \\
\hline Sample ID & \multicolumn{8}{|c|}{ MW-11 } \\
\hline $1 / 8 / 2017$ & 4.35 & 10.7 & 5.69 & 4.92 & 4.72 & 86 & 186.9 & 7.88 \\
\hline $4 / 10 / 2017$ & 4.26 & 16.1 & 5.49 & 2.90 & 4.58 & 114 & -- & 6.68 \\
\hline $7 / 9 / 2017$ & 4.96 & 15.7 & 5.29 & 0.84 & 2.78 & 117 & 294.5 & 7.31 \\
\hline $10 / 28 / 2017$ & 4.77 & 13.2 & 5.07 & 1.21 & 4.04 & 123 & 306.1 & 6.90 \\
\hline $1 / 3 / 2018$ & 4.77 & 12.1 & 5.13 & 2.93 & 4.26 & 28 & 249.1 & 6.90 \\
\hline mean & 4.62 & 13.6 & 5.33 & 2.56 & 4.08 & 94 & 283.2 & 7.13 \\
\hline
\end{tabular}


Table 3. HLDTC Water-Quality Parameters (cont.)

\begin{tabular}{|c|c|c|c|c|c|c|c|c|}
\hline Sample ID & \multicolumn{8}{|c|}{ TF } \\
\hline $1 / 1 / 2017$ & 14.36 & -- & -- & -- & -- & -- & -- & -- \\
\hline $4 / 10 / 2017$ & 14.57 & 15.1 & 6.28 & 5.62 & 5.62 & 87 & -- & 15.78 \\
\hline $7 / 10 / 2017$ & 14.63 & 16.3 & 6.31 & 2.62 & 2.84 & 90 & 249.7 & 14.65 \\
\hline $10 / 28 / 2017$ & 17.10 & 14.5 & 6.05 & 3.56 & -- & 91 & 260.0 & -- \\
\hline $1 / 4 / 2018$ & 4. & 12.9 & 5.99 & 6.11 & 6.56 & 86 & 187.3 & -- \\
\hline mean & 15.06 & 14.7 & 6.16 & 4.48 & 6.56 & 89 & 232.3 & 7.61 \\
\hline Sample ID & \multicolumn{8}{|c|}{ CR } \\
\hline $1 / 8 / 2017$ & -- & 1.5 & 9.26 & 11.80 & 11.78 & 1.5 & -- & -- \\
\hline $4 / 10 / 2017$ & -- & 13.9 & 7.62 & 10.48 & -- & 197 & -- & -- \\
\hline $7 / 12 / 2017$ & -- & 24.9 & 7.80 & 7.32 & 6.90 & 452 & -- & -- \\
\hline $10 / 25 / 2017$ & -- & 13.8 & 8.11 & 9.15 & 8.60 & 372 & -- & -- \\
\hline $1 / 1 / 2018$ & -- & 0.7 & 8.97 & 13.45 & -- & 340 & -- & -_. \\
\hline mean & -- & 11.0 & 8.35 & 10.44 & 5.17 & 272 & -- & \\
\hline
\end{tabular}

Notes:

-- No data; $\mathrm{n} / \mathrm{a}$ (not applicable)

NPDWRs National Primary Drinking Water Regulations - Maximum Contaminant Level

NSDWRs National Secondary Drinking Water Regulations - Secondary Maximum Contaminant Level red Exceeds NPDWRs or NSDWRs

The analytical results for major $(\mathrm{Ca}, \mathrm{Mg}, \mathrm{K}, \mathrm{Na})$ and minor $(\mathrm{Al}, \mathrm{Ba}, \mathrm{Cr}, \mathrm{Co}, \mathrm{Cu}, \mathrm{Fe}$, $\mathrm{Pb}, \mathrm{Li}, \mathrm{Mn}, \mathrm{Ni}, \mathrm{Sr}, \mathrm{V}, \mathrm{Zn}$ ) cations, for which there were laboratory detections, are summarized in Tables 4 and 5, respectively. For major cations, calcium ranged from 0.018 $33.3 \mathrm{mg} / \mathrm{L}$ (mean $6.23 \mathrm{mg} / \mathrm{L}$ ), magnesium from $0.48-17.3 \mathrm{mg} / \mathrm{L}$ (mean $3.43 \mathrm{mg} / \mathrm{L}$ ), potassium from $0.51-11.4 \mathrm{mg} / \mathrm{L}$ (mean $2.73 \mathrm{mg} / \mathrm{L}$ ), and sodium from $0.760-108 \mathrm{mg} / \mathrm{L}$ (mean $13.1 \mathrm{mg} / \mathrm{L}$ ). MW-2 exhibited higher major cation values, compared to other monitoring wells, and concentrations steadily decreased across sampling events. For the major cations, the Cumberland River generally exhibited higher concentrations than the onsite wells (mean: Ca $25.9 \mathrm{mg} / \mathrm{L}, \mathrm{Mg} 12.3 \mathrm{mg} / \mathrm{L} ; \mathrm{K} 2.41 \mathrm{mg} / \mathrm{L}, \mathrm{Na} 22.6 \mathrm{mg} / \mathrm{L}$ ), while exhibiting slightly lower concentrations for the minor cations. 
Wells closer to the ridge (MW-1, MW-3R, MW-8) had the lowest concentrations of cations and the concentrations in wells closer to the river (MW-5, MW-7, MW-10R) were higher. However, unlike the observed water-quality parameter trends, the wells located midway between the ridge and river (MW-4, MW-9R) had the highest cation concentrations. There is no discernable difference between the wells on the eastern (MW10R, MW-11) vs. western (MW-4, MW-5, MW-6, MW-7) portions of the site. Concentrations for iron $(\mathrm{Fe})$ and manganese $(\mathrm{Mn})$ in groundwater exceeded the National Secondary Drinking Water Regulations (NSDWRs) for drinking water. As these are secondary standards, the elevated values do not pose health concerns, but rather aesthetic issues such as potential for staining and pipe scaling.

Table 4. HLDTC Major Cations Analytical Results

\begin{tabular}{|c|c|c|c|c|}
\hline Analyte & $\mathrm{Ca}(\mathrm{ICP})$ & $\mathrm{Mg}(\mathrm{ICP})$ & $\mathrm{K}(\mathrm{ICP})$ & $\mathrm{Na}(\mathrm{ICP})$ \\
\hline MDL (mg/L) & 0.002 & 0.001 & 0.191 & 0.058 \\
\hline NPDWRs - MCL (mg/L) & $\mathrm{n} / \mathrm{a}$ & $\mathrm{n} / \mathrm{a}$ & $\mathrm{n} / \mathrm{a}$ & $\mathrm{n} / \mathrm{a}$ \\
\hline NSDWRs - SMCL (mg/L) & $\mathrm{n} / \mathrm{a}$ & $\mathrm{n} / \mathrm{a}$ & $\mathrm{n} / \mathrm{a}$ & $\mathrm{n} / \mathrm{a}$ \\
\hline Sample ID & & $\mathbf{M}$ & $\mathrm{V}-1$ & \\
\hline $1 / 7 / 2017$ & 1.12 & 0.59 & 1.33 & 1.42 \\
\hline $4 / 7 / 2017$ & 1.13 & 0.52 & 0.75 & 2.58 \\
\hline $7 / 8 / 2017$ & 0.81 & 0.57 & 0.92 & 1.05 \\
\hline $10 / 26 / 2017$ & 0.67 & 0.53 & 0.88 & 0.76 \\
\hline $1 / 2 / 2018$ & 1.14 & 0.48 & 0.94 & 1.83 \\
\hline mean & 0.97 & 0.54 & 0.96 & 1.53 \\
\hline Sample ID & & $\mathbf{M}$ & $V-2$ & \\
\hline $1 / 1 / 2017$ & -- & -- & -- & -- \\
\hline $4 / 8 / 2017$ & 4.41 & 3.14 & 3.12 & 108.0 \\
\hline $7 / 8 / 2017$ & 2.43 & 1.92 & 2.16 & 89.6 \\
\hline $10 / 26 / 2017$ & 2.11 & 1.49 & 2.10 & 54.8 \\
\hline $1 / 2 / 2018$ & 2.01 & 1.22 & 1.92 & 41.6 \\
\hline mean & 2.74 & 1.94 & 2.33 & 73.5 \\
\hline Sample ID & & MY & $-3 R$ & \\
\hline $1 / 6 / 2017$ & 4.23 & 2.73 & 2.24 & 3.99 \\
\hline $4 / 7 / 2017$ & 3.11 & 2.15 & 0.98 & 3.88 \\
\hline $7 / 8 / 2017$ & 3.32 & 2.37 & 1.05 & 4.90 \\
\hline $10 / 26 / 2017$ & 3.34 & 2.29 & 1.07 & 4.68 \\
\hline $1 / 2 / 2018$ & 3.47 & 2.18 & 1.15 & 4.22 \\
\hline mean & 3.49 & 2.34 & 1.30 & 4.33 \\
\hline
\end{tabular}


Table 4. HLDTC Major Cations Analytical Results (cont.)

\begin{tabular}{|c|c|c|c|c|}
\hline \multirow[t]{2}{*}{ Sample ID } & \multicolumn{4}{|c|}{ MW-4 } \\
\hline & 10.7 & 6.39 & 9.81 & 10.8 \\
\hline $4 / 7 / 2017$ & 8.97 & 5.60 & 9.57 & 20.5 \\
\hline $7 / 9 / 2017$ & 9.89 & 6.22 & 9.57 & 20.8 \\
\hline $10 / 28 / 2017$ & 7.76 & 4.93 & 7.64 & 24.6 \\
\hline 10/28/2017 (duplicate) & 7.63 & 4.89 & 7.57 & 24.2 \\
\hline $1 / 5 / 2018$ & 7.33 & 4.13 & 6.76 & 21.9 \\
\hline mean & 8.71 & 5.36 & 8.49 & 20.5 \\
\hline Sample ID & & MV & & \\
\hline $1 / 6 / 2017$ & 5.79 & 3.80 & 5.06 & 12.2 \\
\hline $4 / 9 / 2017$ & 3.36 & 1.42 & 1.59 & 5.57 \\
\hline $7 / 10 / 2017$ & 5.11 & 3.31 & 4.08 & 8.34 \\
\hline $10 / 27 / 2017$ & 4.58 & 2.75 & 3.41 & 7.44 \\
\hline $1 / 4 / 2018$ & 4.19 & 2.38 & 3.08 & 6.30 \\
\hline mean & 4.61 & 2.73 & 3.44 & 7.97 \\
\hline Sample ID & & MV & & \\
\hline $1 / 5 / 2017$ & 12.9 & 5.06 & 2.39 & 11.9 \\
\hline $4 / 9 / 2017$ & 10.4 & 4.18 & 1.58 & 10.3 \\
\hline 4/9/2017 (duplicate) & 10.5 & 4.20 & 1.50 & 10.3 \\
\hline $7 / 10 / 2017$ & 10.3 & 4.38 & 1.61 & 10.2 \\
\hline $10 / 27 / 2017$ & 10.5 & 4.34 & 1.64 & 10.4 \\
\hline $1 / 5 / 2018$ & 10.2 & 3.81 & 1.63 & 9.40 \\
\hline 1/5/2018 (duplicate) & 9.82 & 3.73 & 1.47 & 9.23 \\
\hline mean & 10.66 & 4.24 & 1.69 & 10.25 \\
\hline Sample ID & & MV & & \\
\hline $1 / 5 / 2017$ & 3.09 & 1.93 & 1.17 & 6.72 \\
\hline $4 / 9 / 2017$ & 2.80 & 1.85 & 0.70 & 5.86 \\
\hline $7 / 10 / 2017$ & 2.99 & 1.99 & 0.65 & 5.69 \\
\hline 7/10/2017 (duplicate) & 3.02 & 2.02 & 0.66 & 5.77 \\
\hline $10 / 27 / 2017$ & 3.27 & 2.11 & 0.80 & 5.78 \\
\hline $1 / 5 / 2018$ & 3.40 & 1.93 & 0.91 & 5.74 \\
\hline mean & 3.10 & 1.97 & 0.82 & 5.93 \\
\hline Sample ID & & MV & & \\
\hline $1 / 7 / 2017$ & 0.88 & 0.60 & 1.44 & 0.95 \\
\hline $4 / 8 / 2017$ & 0.88 & 0.64 & 1.39 & 1.21 \\
\hline $7 / 9 / 2017$ & 0.83 & 0.62 & 1.47 & 1.82 \\
\hline $10 / 27 / 2017$ & 0.90 & 0.59 & 1.59 & 2.31 \\
\hline $1 / 3 / 2018$ & 1.58 & 0.55 & 1.78 & 2.35 \\
\hline mean & 1.01 & 0.60 & 1.53 & 1.73 \\
\hline
\end{tabular}


Table 4. HLDTC Major Cations Analytical Results (cont.)

\begin{tabular}{|c|c|c|c|c|}
\hline Sample ID & & MW & & \\
\hline $1 / 7 / 2017$ & 4.89 & 3.91 & 11.4 & 8.13 \\
\hline 4/9/2017 & 6.69 & 5.17 & 9.77 & 8.17 \\
\hline $7 / 8 / 2017$ & 7.72 & 6.36 & 7.68 & 7.91 \\
\hline $10 / 26 / 2017$ & 7.85 & 6.19 & 7.07 & 6.69 \\
\hline $1 / 4 / 2018$ & 7.61 & 5.48 & 7.17 & 6.34 \\
\hline mean & 6.95 & 5.42 & 8.62 & 7.45 \\
\hline Sample ID & & MW & & \\
\hline $1 / 8 / 2017$ & 0.57 & 0.61 & 0.57 & 2.74 \\
\hline 1/8/2017 (duplicate) & 0.54 & 0.61 & 0.74 & 2.23 \\
\hline $4 / 8 / 2017$ & 0.34 & 0.60 & 0.51 & 1.97 \\
\hline 7/9/2017 & 0.23 & 0.68 & 0.52 & 2.05 \\
\hline $10 / 27 / 2017$ & 0.18 & 0.62 & 0.78 & 2.11 \\
\hline $1 / 1 / 2018$ & 0.32 & 0.61 & 0.87 & 2.33 \\
\hline mean & 0.36 & 0.62 & 0.67 & 2.24 \\
\hline Sample ID & & MV & & \\
\hline $1 / 8 / 2017$ & 3.38 & 1.81 & 1.24 & 12.9 \\
\hline $4 / 10 / 2017$ & 3.27 & 2.23 & 1.15 & 18.0 \\
\hline 7/9/2017 & 3.44 & 2.85 & 1.24 & 18.5 \\
\hline $10 / 28 / 2017$ & 3.36 & 2.83 & 1.27 & 20.7 \\
\hline $1 / 3 / 2018$ & 3.26 & 2.30 & 1.55 & 21.5 \\
\hline mean & 3.34 & 2.40 & 1.29 & 18.3 \\
\hline Sample ID & & $\mathbf{T}$ & & \\
\hline $1 / 1 / 2017$ & -- & -- & -- & -- \\
\hline $4 / 10 / 2017$ & 7.84 & 3.83 & 1.76 & 7.81 \\
\hline $7 / 10 / 2017$ & 8.23 & 4.19 & 1.79 & 8.02 \\
\hline $10 / 28 / 2017$ & 8.73 & 4.27 & 1.85 & 8.27 \\
\hline $1 / 4 / 2018$ & 8.65 & 3.82 & 1.90 & 7.80 \\
\hline mean & 8.36 & 4.03 & 1.83 & 7.98 \\
\hline Sample ID & & C & & \\
\hline $1 / 8 / 2017$ & 24.9 & 12.1 & 1.97 & 19.7 \\
\hline $4 / 10 / 2017$ & 21.1 & 10.2 & 1.71 & 15.0 \\
\hline $7 / 12 / 2017$ & 33.3 & 17.3 & 2.97 & 33.8 \\
\hline $10 / 25 / 2017$ & 24.5 & 10.6 & 3.26 & 24.3 \\
\hline $1 / 1 / 2018$ & 25.9 & 11.4 & 2.13 & 20.3 \\
\hline mean & 25.9 & 12.3 & 2.41 & 22.6 \\
\hline
\end{tabular}

Notes:

-- No data; $\mathrm{n} / \mathrm{a}$ (not applicable)

ICP Dissolved by ICP (Inductively Coupled Plasma)

MDL Method Detection Limit

NPDWRs National Primary Drinking Water Regulations -

Maximum Contaminant Level

NSDWRs National Secondary Drinking Water Regulations -

Secondary Maximum Contaminant Level 
Table 5. HLDTC Minor Cations Analytical Results

\begin{tabular}{|c|c|c|c|c|c|c|c|c|c|c|c|c|c|}
\hline Analyte & $\begin{array}{l}\text { Al } \\
(\mathrm{ICP})\end{array}$ & $\begin{array}{l}\mathrm{Ba} \\
(\mathrm{ICP})\end{array}$ & $\begin{array}{l}\mathrm{Cr} \\
(\mathrm{ICP})\end{array}$ & $\begin{array}{l}\text { Co } \\
\text { (ICP) }\end{array}$ & $\begin{array}{l}\mathrm{Cu} \\
(\mathrm{ICP})\end{array}$ & $\begin{array}{l}\mathrm{Fe} \\
(\mathrm{ICP})\end{array}$ & $\begin{array}{l}\mathrm{Pb} \\
(\mathrm{ICP})\end{array}$ & $\begin{array}{l}\mathrm{Li} \\
(\mathrm{ICP})\end{array}$ & $\begin{array}{l}\mathrm{Mn} \\
\text { (ICP) }\end{array}$ & $\begin{array}{l}\mathrm{Ni} \\
(\mathrm{ICP})\end{array}$ & $\begin{array}{l}\mathrm{Sr} \\
(\mathrm{ICP})\end{array}$ & $\begin{array}{l}\mathrm{V} \\
\text { (ICP) }\end{array}$ & $\begin{array}{l}\mathrm{Zn} \\
(\mathrm{ICP})\end{array}$ \\
\hline $\begin{array}{l}\text { MDL } \\
(\mathrm{mg} / \mathrm{L})\end{array}$ & 0.061 & 0.003 & 0.024 & 0.001 & 0.005 & 0.002 & 0.01 & 0.001 & 0.001 & 0.002 & 0.01 & 0.008 & 0.002 \\
\hline $\begin{array}{l}\text { NPDWRs } \\
-\mathrm{MCL} \\
\text { (mg/L) } \\
\text { NSDWRs }\end{array}$ & $\mathrm{n}$ & 2.0 & 0.1 & $\mathrm{n} / \mathrm{a}$ & 然 & $\mathrm{n} / \mathrm{a}$ & 0.02 & $\mathrm{n} / \mathrm{a}$ & $\mathrm{n} / \mathrm{a}$ & $/ \mathrm{a}$ & $\mathrm{n} / \mathrm{a}$ & $\mathrm{n} / \mathrm{a}$ & $\mathrm{n} / \mathrm{a}$ \\
\hline $\begin{array}{l}-\mathrm{SMCL} \\
(\mathrm{mg} / \mathrm{L})\end{array}$ & $\begin{array}{r}0.05- \\
0.2\end{array}$ & $\mathrm{n}$ & $\mathrm{n} / \mathrm{a}$ & & 1 & 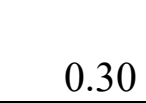 & $\mathrm{n} / \mathrm{a}$ & $\mathrm{n} / \mathrm{a}$ & 0.05 & $\mathrm{n}$ & $\mathrm{n} / \mathrm{s}$ & $\mathrm{n} / \mathrm{a}$ & 5.000 \\
\hline Sample ID & & & & & & & & & & & & & \\
\hline & UDL & 020 & MDL & & MDL & MDL & DL & & & & & & 10 \\
\hline 4 & MDL & 020 & $<\mathrm{MDL}$ & 0.003 & MDL & 0.02 & $<\mathrm{MDL}$ & $<\mathrm{MDL}$ & 0.06 & $<\mathrm{MDL}$ & $\mathrm{L}$ & DL & 0.008 \\
\hline 7 & MDL & 0.020 & $<\mathrm{MDL}$ & 0.002 & MDL & 0.01 & $<\mathrm{MDL}$ & $<\mathrm{MDL}$ & 0.06 & $<\mathrm{MDL}$ & & $<\mathrm{N}$ & 0.020 \\
\hline $10 / 2$ & MDL & 0.020 & $<\mathrm{MDL}$ & & MDL & & $<\mathrm{MDL}$ & & & & & $<\mathrm{MDL}$ & 0.060 \\
\hline $1 / 2 / 2018$ & $<\mathrm{MDL}$ & 0.020 & $<\mathrm{MDL}$ & $<\mathrm{MDL}$ & $\mathrm{MDL}$ & $<\mathrm{MDL}$ & $<\mathrm{MDL}$ & 0.003 & 0.02 & $<\mathrm{MDL}$ & $<\mathrm{MDL}$ & $<\mathrm{MDL}$ & 0.009 \\
\hline mean & $<\mathrm{MDL}$ & 0.020 & $<\mathrm{MDL}$ & 0.003 & $<\mathrm{MDL}$ & 0.01 & $<\mathrm{MDL}$ & 0.003 & 0.06 & 0.003 & 0.01 & $<\mathrm{MDL}$ & 0.021 \\
\hline & & & & & & & MW-2 & & & & & & \\
\hline $1 /$ & -- & -- & -- & -- & -- & -- & -- & -- & -- & -- & -- & -- & -- \\
\hline & IDL & 40 & $\mathrm{MDL}$ & & MDL & & & & 0.59 & [DL & 04 & $\mathrm{MDL}$ & $<\mathrm{MDL}$ \\
\hline 7 & UDL & & $<\mathrm{MDL}$ & & MDL & & & $<\mathrm{MDL}$ & & & 0.02 & & 0.005 \\
\hline $10 / 26 / 2017$ & $<\mathrm{MDL}$ & 0.020 & $<\mathrm{MDL}$ & 0.001 & $<\mathrm{MDL}$ & 0.18 & $<\mathrm{MDL}$ & 0.005 & 0.12 & $<\mathrm{MDL}$ & 0.02 & $<\mathrm{MDL}$ & 0.030 \\
\hline $1 / 2 / 2018$ & $<\mathrm{MDL}$ & 0.010 & $<\mathrm{MDL}$ & $<\mathrm{MDL}$ & $<\mathrm{MDL}$ & & $<\mathrm{MDL}$ & & 0.07 & $<\mathrm{MDL}$ & 0.02 & $<\mathrm{MDL}$ & 0.010 \\
\hline mean & $<\mathrm{MDL}$ & 0.023 & $<\mathrm{MDL}$ & 0.005 & $<\mathrm{MDL}$ & 0.15 & $<\mathrm{MDL}$ & 0.005 & 0.25 & $<\mathrm{MDL}$ & 0.03 & $<\mathrm{MDL}$ & 0.015 \\
\hline
\end{tabular}


Table 5. HLDTC Minor Cations Analytical Results (cont.)

\begin{tabular}{|c|c|c|c|c|c|c|c|c|c|c|c|c|c|}
\hline Sample ID & \multicolumn{13}{|c|}{ MW-3R } \\
\hline $1 / 6 / 2017$ & $<\mathrm{MDL}$ & 0.070 & $<\mathrm{MDL}$ & $<\mathrm{MDL}$ & $<\mathrm{MDL}$ & 7.70 & $<\mathrm{MDL}$ & 0.010 & 0.60 & $<\mathrm{MDL}$ & 0.06 & $<\mathrm{MDL}$ & 0.015 \\
\hline $4 / 7 / 2017$ & $<\mathrm{MDL}$ & 0.060 & $<\mathrm{MDL}$ & $<\mathrm{MDL}$ & $<\mathrm{MDL}$ & 8.55 & $<\mathrm{MDL}$ & 0.004 & 0.49 & $<\mathrm{MDL}$ & 0.04 & $<\mathrm{MDL}$ & 0.140 \\
\hline $7 / 8 / 2017$ & $<\mathrm{MDL}$ & 0.050 & $<\mathrm{MDL}$ & $<\mathrm{MDL}$ & $<\mathrm{MDL}$ & 8.69 & $<\mathrm{MDL}$ & 0.002 & 0.49 & $<\mathrm{MDL}$ & 0.04 & $<\mathrm{MDL}$ & $<\mathrm{MDL}$ \\
\hline $10 / 26 / 2017$ & $<\mathrm{MDL}$ & 0.060 & $<\mathrm{MDL}$ & $<\mathrm{MDL}$ & $<\mathrm{MDL}$ & 9.45 & $<\mathrm{MDL}$ & 0.006 & 0.49 & $<\mathrm{MDL}$ & 0.05 & $<\mathrm{MDL}$ & 0.002 \\
\hline $1 / 2 / 2018$ & $<\mathrm{MDL}$ & 0.060 & $<\mathrm{MDL}$ & $<\mathrm{MDL}$ & $<\mathrm{MDL}$ & 10.1 & $<\mathrm{MDL}$ & 0.008 & 0.49 & $<\mathrm{MDL}$ & 0.05 & 0.010 & $<\mathrm{MDL}$ \\
\hline mean & $<\mathrm{MDL}$ & 0.060 & $<\mathrm{MDL}$ & $<\mathrm{MDL}$ & $<\mathrm{MDL}$ & 8.90 & $<\mathrm{MDL}$ & 0.006 & 0.51 & $<\mathrm{MDL}$ & 0.05 & $<\mathrm{MDL}$ & 0.523 \\
\hline Sample ID & & & & & & & MW-4 & & & & & & \\
\hline $1 / 6 / 2017$ & $<\mathrm{MDL}$ & 0.040 & $<\mathrm{MDL}$ & 0.020 & $<\mathrm{MDL}$ & 1.48 & $<\mathrm{MDL}$ & 0.004 & 2.28 & 0.002 & 0.07 & $<\mathrm{MDL}$ & 0.010 \\
\hline $4 / 7 / 2017$ & $<\mathrm{MDL}$ & 0.040 & $<\mathrm{MDL}$ & 0.020 & $<\mathrm{MDL}$ & 1.05 & $<\mathrm{MDL}$ & $<\mathrm{MDL}$ & 2.52 & 0.002 & 0.06 & $<\mathrm{MDL}$ & 0.020 \\
\hline $7 / 9 / 2017$ & $<\mathrm{MDL}$ & 0.070 & $<\mathrm{MDL}$ & 0.010 & $<\mathrm{MDL}$ & 1.39 & $<\mathrm{MDL}$ & 0.002 & 2.59 & $<\mathrm{MDL}$ & .06 & $<\mathrm{MDL}$ & 0.080 \\
\hline $10 / 28 / 2017$ & $<\mathrm{MDL}$ & 0.040 & $<\mathrm{MDL}$ & 0.015 & $<\mathrm{MDL}$ & 2.25 & $<\mathrm{MDL}$ & 0.006 & 2.16 & 0.02 & 0.05 & $<\mathrm{MDL}$ & 0.010 \\
\hline $10 / 28 / 2017$ & & & & & & & & & & & & & \\
\hline (duplicate) & $<\mathrm{MDL}$ & 40 & $<\mathrm{MDL}$ & 0. & IVIDL & 2.19 & $<\mathrm{MDL}$ & $<\mathrm{M}$ & .14 & $<\mathrm{MDL}$ & 5 & $<\mathrm{MDL}$ & 0.009 \\
\hline $1 / 5 / 2018$ & $<\mathrm{MDL}$ & 0.030 & $<\mathrm{MDL}$ & 0.010 & $<\mathrm{MDL}$ & 1.71 & $<\mathrm{MDL}$ & 0.004 & 1.78 & $<\mathrm{MDL}$ & 0.05 & $<\mathrm{MDL}$ & 0.009 \\
\hline mean & $<\mathrm{MDL}$ & 0.043 & $<\mathrm{MDL}$ & 0.015 & $<\mathrm{MDL}$ & 1.68 & $<\mathrm{MDL}$ & 0.004 & 2.25 & $<\mathrm{MDL}$ & 0.06 & $<\mathrm{MDL}$ & 0.023 \\
\hline Sample ID & & & & & & & MW-5 & & & & & & \\
\hline $1 / 6 / 2017$ & 0.630 & 0.090 & $<\mathrm{MDL}$ & 0.020 & 0.008 & 23.5 & $<\mathrm{MDL}$ & 0.004 & 3.71 & 0.006 & 0.06 & $<\mathrm{MDL}$ & 0.020 \\
\hline $4 / 9 / 2017$ & $<\mathrm{MDL}$ & 0.070 & $<\mathrm{MDL}$ & 0.004 & $<\mathrm{MDL}$ & 22.9 & $<\mathrm{MDL}$ & 0.003 & 2.13 & $<\mathrm{MDL}$ & 0.03 & $<\mathrm{MDL}$ & 0.030 \\
\hline $7 / 10 / 2017$ & $<\mathrm{MDL}$ & 0.070 & $<\mathrm{MDL}$ & 0.009 & $<\mathrm{MDL}$ & 21.1 & $<\mathrm{MDL}$ & 0.004 & 3.27 & 0.002 & 0.05 & $<\mathrm{MDL}$ & 0.150 \\
\hline $10 / 27 / 2017$ & $<\mathrm{MDL}$ & 0.070 & $<\mathrm{MDL}$ & 0.005 & 0.025 & 21.9 & $<\mathrm{MDL}$ & 0.005 & 2.87 & 0.002 & 0.05 & $<\mathrm{MDL}$ & 0.010 \\
\hline $1 / 4 / 2018$ & $<\mathrm{MDL}$ & 0.060 & $<\mathrm{MDL}$ & 0.008 & $<\mathrm{MDL}$ & 23.0 & $<\mathrm{MDL}$ & 0.006 & 2.67 & 0.003 & 0.05 & 0.010 & 0.004 \\
\hline mean & 0.630 & 0.072 & $<\mathrm{MDL}$ & 0.009 & 0.017 & 22.5 & $<\mathrm{MDL}$ & 0.004 & 2.93 & 0.003 & 0.05 & 0.010 & 0.043 \\
\hline
\end{tabular}


Table 5. HLDTC Minor Cations Analytical Results (cont.)

\begin{tabular}{|c|c|c|c|c|c|c|c|c|c|c|c|c|c|}
\hline \multicolumn{7}{|l|}{ Sample ID } & \multicolumn{7}{|l|}{ MW-6 } \\
\hline $1 / 5 / 2017$ & MDL & 0.160 & $<\mathrm{MDL}$ & $<\mathrm{MDL}$ & $<\mathrm{MDL}$ & 28.0 & $<\mathrm{MDL}$ & 0.002 & 0.37 & $<\mathrm{MDL}$ & 0.38 & $<\mathrm{MDL}$ & 0.004 \\
\hline $4 / 9 / 2017$ & $<\mathrm{MDL}$ & 0.180 & $<\mathrm{MDL}$ & $<\mathrm{MDL}$ & $<\mathrm{MDL}$ & 26.9 & $<\mathrm{MDL}$ & $<\mathrm{MDL}$ & 0.35 & $<\mathrm{MDL}$ & 0.27 & $<\mathrm{MDL}$ & 0.290 \\
\hline $4 / 9 / 2017$ & & & & & & & & & & & & & \\
\hline (duplicate) & MDL & 0.170 & $<\mathrm{MDL}$ & $<\mathrm{MDL}$ & $<\mathrm{MDL}$ & 27.9 & $<\mathrm{MDL}$ & $<\mathrm{MDL}$ & 0.35 & $<\mathrm{MDL}$ & 0.28 & $<\mathrm{MDL}$ & 0.060 \\
\hline $7 / 10 / 2017$ & $<\mathrm{MDL}$ & 0.170 & $<\mathrm{MDL}$ & $<\mathrm{MDL}$ & $<\mathrm{MDL}$ & 0.7 & $<\mathrm{MDL}$ & 0.003 & 0.35 & $<\mathrm{MDL}$ & 0.24 & $<\mathrm{MDL}$ & $<\mathrm{MDL}$ \\
\hline $10 / 27 / 2017$ & $<\mathrm{MDL}$ & 0.180 & $<\mathrm{MDL}$ & $<\mathrm{MDL}$ & 0.005 & 33.8 & $<\mathrm{MDL}$ & 0.004 & 0.35 & 0.003 & 0.26 & $<\mathrm{MDL}$ & 0.009 \\
\hline $1 / 5 / 2018$ & $<\mathrm{MDL}$ & 0.180 & $<\mathrm{MDL}$ & $<\mathrm{MDL}$ & $<\mathrm{MDL}$ & 39.4 & $<\mathrm{MDL}$ & 0.008 & 0.36 & 0.002 & 0.24 & $<\mathrm{MDL}$ & 0.004 \\
\hline $1 / 5 / 2018$ & & & & & & & & & & & & & \\
\hline (duplicate) & $<\mathrm{MDL}$ & 0.180 & $<\mathrm{MDL}$ & $<\mathrm{MDL}$ & $<\mathrm{MDL}$ & 38.0 & $<\mathrm{MDL}$ & 0.004 & 0.35 & $<\mathrm{MDL}$ & 0.24 & $<\mathrm{MDL}$ & 0.003 \\
\hline mean & $<\mathrm{MDL}$ & 0.170 & $<\mathrm{MDL}$ & $<\mathrm{MDL}$ & 0.005 & 2.1 & $<\mathrm{MDL}$ & 0.004 & 0.35 & 0.003 & 0.27 & $<\mathrm{MDL}$ & 0.061 \\
\hline Sample ID & & & & & & & MW-7 & & & & & & \\
\hline $1 / 5 / 2017$ & 0.12 & .090 & $<\mathrm{MDL}$ & $<\mathrm{MDL}$ & $<\mathrm{MDL}$ & 32.7 & $<\mathrm{MDL}$ & 0.002 & 1.64 & 0.002 & 0.05 & $<\mathrm{MDL}$ & 0.010 \\
\hline $4 / 9 / 2017$ & $<\mathrm{MDL}$ & 0.090 & $<\mathrm{MDL}$ & $<\mathrm{MDL}$ & $<\mathrm{MDL}$ & 34.3 & $<\mathrm{MDL}$ & $<\mathrm{MDL}$ & 1.84 & $<\mathrm{MDL}$ & 0.04 & $<\mathrm{MDL}$ & 0.150 \\
\hline $7 / 10 / 2017$ & $<\mathrm{MDL}$ & 0.080 & $<\mathrm{MDL}$ & $<\mathrm{MDL}$ & $<\mathrm{MDL}$ & 32.1 & $<\mathrm{MDL}$ & 0.001 & 1.93 & $<\mathrm{MDL}$ & 0.04 & $<\mathrm{MDL}$ & 0.030 \\
\hline $7 / 10 /$ & & & & & & & & & & & & & \\
\hline (duplicate) & $<\mathrm{MDL}$ & 0 & $<\mathrm{MDL}$ & $<\mathrm{MDL}$ & $<\mathrm{MDL}$ & 32.1 & $<\mathrm{MDL}$ & 0 & 1.88 & $<\mathrm{MDL}$ & 0.04 & $<\mathrm{MDL}$ & 0.020 \\
\hline $10 / 27 / 2017$ & $<\mathrm{MDL}$ & 0.090 & $<\mathrm{MDL}$ & $<\mathrm{MDL}$ & $<\mathrm{MDL}$ & 36.8 & $<\mathrm{MDL}$ & 0.003 & 2.05 & 0.003 & 0.04 & $<\mathrm{MDL}$ & 0.008 \\
\hline $1 / 5 / 2018$ & $<\mathrm{MDL}$ & 0.090 & $<\mathrm{MDL}$ & $<\mathrm{MDL}$ & $<\mathrm{MDL}$ & 38.6 & $<\mathrm{MDL}$ & 0.006 & 1.92 & $<\mathrm{MDL}$ & 0.05 & $<\mathrm{MDL}$ & 0.007 \\
\hline mean & 0.12 & 0.087 & $<\mathrm{MDL}$ & $<\mathrm{MDL}$ & $<\mathrm{MDL}$ & 34.4 & $<\mathrm{MDL}$ & 0.003 & 1.88 & 0.003 & 0.04 & $<\mathrm{MDL}$ & 0.038 \\
\hline Sample ID & & & & & & & MW-8 & & & & & & \\
\hline $1 / 7 / 2017$ & $<\mathrm{MDL}$ & 0.020 & $<\mathrm{MDL}$ & $<\mathrm{MDL}$ & $<\mathrm{MDL}$ & 0.17 & $<\mathrm{MDL}$ & $<\mathrm{MDL}$ & 0.11 & 0.003 & MDL & $<\mathrm{MDL}$ & 0.010 \\
\hline $4 / 8 / 2017$ & $<\mathrm{MDL}$ & 0.030 & $<\mathrm{MDL}$ & 0.001 & $<\mathrm{MDL}$ & 0.63 & $<\mathrm{MDL}$ & $<\mathrm{MDL}$ & 0.14 & $<\mathrm{MDL}$ & $<\mathrm{MDL}$ & $<\mathrm{MDL}$ & 0.410 \\
\hline $7 / 9 / 2017$ & $<\mathrm{MDL}$ & 0.030 & $<\mathrm{MDL}$ & 0.001 & $<\mathrm{MDL}$ & 1.14 & $<\mathrm{MDL}$ & 0.002 & 0.14 & $<\mathrm{MDL}$ & $<\mathrm{MDL}$ & $<\mathrm{MDL}$ & 0.100 \\
\hline $10 / 27 / 2017$ & $<\mathrm{MDL}$ & 0.020 & 0.030 & 0.001 & $<\mathrm{MDL}$ & 1.90 & $<\mathrm{MDL}$ & 0.005 & 0.16 & 0.008 & $<\mathrm{MDL}$ & $<\mathrm{MDL}$ & 0.010 \\
\hline $1 / 3 / 2018$ & $<\mathrm{MDL}$ & 0.015 & $<\mathrm{MDL}$ & 0.001 & $<\mathrm{MDL}$ & 0.44 & $<\mathrm{MDL}$ & 0.008 & 0.17 & 0.002 & $<\mathrm{MDL}$ & $<\mathrm{MDL}$ & 0.009 \\
\hline mean & $<\mathrm{MDL}$ & 0.023 & 0.030 & 0.001 & $<\mathrm{MDL}$ & 0.86 & $<\mathrm{MDL}$ & 0.005 & 0.14 & 0.004 & $<\mathrm{MDL}$ & $<\mathrm{MDL}$ & 0.108 \\
\hline
\end{tabular}


Table 5. HLDTC Minor Cations Analytical Results (cont.)

\begin{tabular}{|c|c|c|c|c|c|c|c|c|c|c|c|c|c|}
\hline Sample ID & \multicolumn{13}{|c|}{ MW-9R } \\
\hline $1 / 7 / 2017$ & $<\mathrm{MDL}$ & 0.170 & $<\mathrm{MDL}$ & $<\mathrm{MDL}$ & $<\mathrm{MDL}$ & 3.25 & $<\mathrm{MDL}$ & 0.004 & 1.54 & 0.002 & 0.08 & $<\mathrm{MDL}$ & 0.015 \\
\hline $4 / 9 / 2017$ & $<\mathrm{MDL}$ & 0.050 & $<\mathrm{MDL}$ & 0.004 & $<\mathrm{MDL}$ & 1.25 & $<\mathrm{MDL}$ & 0.004 & 1.42 & 0.003 & 0.10 & $<\mathrm{MDL}$ & 0.009 \\
\hline $7 / 8 / 2017$ & $<\mathrm{MDL}$ & 0.050 & $<\mathrm{MDL}$ & 0.005 & $<\mathrm{MDL}$ & 1.21 & $<\mathrm{MDL}$ & 0.007 & 1.31 & $<\mathrm{MDL}$ & 0.11 & $<\mathrm{MDL}$ & 0.020 \\
\hline $10 / 26 / 2017$ & $<\mathrm{MDL}$ & 0.060 & $<\mathrm{MDL}$ & 0.006 & $<\mathrm{MDL}$ & 2.14 & $<\mathrm{MDL}$ & 0.010 & 1.26 & 0.003 & 0.13 & $<\mathrm{MDL}$ & 0.016 \\
\hline $1 / 4 / 2018$ & $<\mathrm{MDL}$ & 0.050 & $<\mathrm{MDL}$ & 0.003 & $<\mathrm{MDL}$ & 1.44 & $<\mathrm{MDL}$ & 0.006 & 1.45 & 0.003 & 0.12 & $<\mathrm{MDL}$ & 0.007 \\
\hline mean & $<\mathrm{MDL}$ & 0.076 & $<\mathrm{MDL}$ & 0.005 & $<\mathrm{MDL}$ & 1.86 & $<\mathrm{MDL}$ & 0.006 & 1.40 & 0.003 & 0.11 & $<\mathrm{MDL}$ & 0.013 \\
\hline Sample ID & \multicolumn{13}{|c|}{ MW-10R } \\
\hline $1 / 8 / 2017$ & $<\mathrm{MDL}$ & 0.010 & $<\mathrm{MDL}$ & $<\mathrm{MDL}$ & $<\mathrm{MDL}$ & 0.12 & $<\mathrm{MDL}$ & $<\mathrm{MDL}$ & 0.14 & 0.002 & $\mathrm{MDL}$ & $<\mathrm{MDL}$ & 0.010 \\
\hline $1 / 8 / 2017$ & & & & & & & & & & & & & \\
\hline (duplicate) & $<\mathrm{MDL}$ & 0.010 & $<\mathrm{MDL}$ & $<\mathrm{MDL}$ & $<\mathrm{MDL}$ & 0.18 & $<\mathrm{MDL}$ & 0.004 & 0.13 & 0.002 & $<\mathrm{MDL}$ & $<\mathrm{MDL}$ & 0.010 \\
\hline $4 / 8 / 2017$ & $<\mathrm{MDL}$ & 0.015 & $<\mathrm{MDL}$ & 0.010 & $<\mathrm{MDL}$ & 0.22 & $<\mathrm{MDL}$ & $<\mathrm{MDL}$ & 0.41 & 0.004 & $<\mathrm{MDL}$ & $<\mathrm{MDL}$ & .060 \\
\hline $7 / 9 / 2017$ & $<\mathrm{MDL}$ & 0.050 & $<\mathrm{MDL}$ & 0.010 & $<\mathrm{MDL}$ & 0.01 & $<\mathrm{MDL}$ & 0.002 & 0.32 & 0.003 & $<\mathrm{MDL}$ & $<\mathrm{MDL}$ & 0.110 \\
\hline $10 / 27 / 2017$ & $<\mathrm{MDL}$ & 0.010 & $<\mathrm{MDL}$ & 0.007 & $<\mathrm{MDL}$ & 0.38 & $<\mathrm{MDL}$ & $<\mathrm{MDL}$ & 0.23 & 0.003 & $<\mathrm{MDL}$ & $<\mathrm{MDL}$ & 0.010 \\
\hline $1 / 1 / 2018$ & $<\mathrm{MDL}$ & 0.010 & $<\mathrm{MDL}$ & 0.009 & $<\mathrm{MDL}$ & 0.32 & $<\mathrm{MDL}$ & 0.001 & 0.32 & 0.004 & $<\mathrm{MDL}$ & $<\mathrm{MDL}$ & 0.006 \\
\hline mean & $<\mathrm{MDL}$ & 0.018 & $<\mathrm{MDL}$ & 0.009 & $<\mathrm{MDL}$ & 0.21 & $<\mathrm{MDL}$ & 0.002 & 0.26 & 0.003 & $<\mathrm{MDL}$ & $<\mathrm{MDL}$ & 0.034 \\
\hline Sample ID & \multicolumn{13}{|c|}{ MW-11 } \\
\hline $1 / 8 / 2017$ & 0.190 & 0.030 & $<\mathrm{MDL}$ & $<\mathrm{MDL}$ & $<\mathrm{MDL}$ & 0.24 & $<\mathrm{MDL}$ & 0.008 & 0.17 & 0.005 & 0.03 & $<\mathrm{MDL}$ & 0.040 \\
\hline $4 / 10 / 2017$ & $<\mathrm{MDL}$ & 0.040 & $<\mathrm{MDL}$ & 0.010 & $<\mathrm{MDL}$ & 0.02 & $<\mathrm{MDL}$ & 0.001 & 0.18 & 0.010 & 0.03 & $<\mathrm{MDL}$ & 0.030 \\
\hline 7/9/2017 & $<\mathrm{MDL}$ & 0.060 & $<\mathrm{MDL}$ & 0.010 & $<\mathrm{MDL}$ & 0.04 & $<\mathrm{MDL}$ & $<\mathrm{MDL}$ & 0.13 & 0.010 & 0.03 & $<\mathrm{MDL}$ & 0.050 \\
\hline $10 / 28 / 2017$ & $<\mathrm{MDL}$ & 0.060 & $<\mathrm{MDL}$ & 0.007 & $<\mathrm{MDL}$ & 0.02 & $<\mathrm{MDL}$ & 0.003 & 0.10 & 0.007 & 0.03 & $<\mathrm{MDL}$ & 0.030 \\
\hline $1 / 3 / 2018$ & $<\mathrm{MDL}$ & 0.060 & $<\mathrm{MDL}$ & 0.005 & $<\mathrm{MDL}$ & $<\mathrm{MDL}$ & $<\mathrm{MDL}$ & 0.006 & 0.07 & 0.006 & 0.03 & $<\mathrm{MDL}$ & 0.020 \\
\hline mean & 0.190 & 0.050 & $<\mathrm{MDL}$ & 0.008 & $<\mathrm{MDL}$ & 0.08 & $<\mathrm{MDL}$ & 0.005 & 0.13 & 0.008 & 0.03 & $<\mathrm{MDL}$ & 0.034 \\
\hline Sample ID & \multicolumn{13}{|c|}{ TF } \\
\hline $1 / 1 / 2017$ & -- & -- & -- & -- & -- & -- & -- & -- & -- & -- & -- & -- & -- \\
\hline $4 / 10 / 2017$ & 0.070 & 0.260 & $<\mathrm{MDL}$ & $<\mathrm{MDL}$ & $<\mathrm{MDL}$ & 0.16 & 0.01 & 0.010 & 0.13 & $<\mathrm{MDL}$ & 0.26 & $<\mathrm{MDL}$ & 0.090 \\
\hline $7 / 10 / 2017$ & $<\mathrm{MDL}$ & 0.280 & $<\mathrm{MDL}$ & $<\mathrm{MDL}$ & $<\mathrm{MDL}$ & 0.23 & $<\mathrm{MDL}$ & 0.010 & 0.13 & $<\mathrm{MDL}$ & 0.25 & $<\mathrm{MDL}$ & 0.030 \\
\hline $10 / 28 / 2017$ & $<\mathrm{MDL}$ & 0.280 & $<\mathrm{MDL}$ & $<\mathrm{MDL}$ & $<\mathrm{MDL}$ & 0.22 & $<\mathrm{MDL}$ & 0.020 & 0.14 & 0.002 & 0.28 & $<\mathrm{MDL}$ & 0.005 \\
\hline $1 / 4 / 2018$ & $<\mathrm{MDL}$ & 0.280 & $<\mathrm{MDL}$ & $<\mathrm{MDL}$ & $<\mathrm{MDL}$ & 0.38 & $<\mathrm{MDL}$ & 0.020 & 0.14 & $<\mathrm{MDL}$ & 0.29 & 0.010 & 0.007 \\
\hline mean & 0.07 & 0.280 & $<\mathrm{MDL}$ & $<\mathrm{MDL}$ & $<\mathrm{MDL}$ & 0.25 & 0.01 & 0.015 & 0.14 & 0.002 & 0.27 & 0.010 & 0.033 \\
\hline
\end{tabular}


Table 5. HLDTC Minor Cations Analytical Results (cont.)

\begin{tabular}{|c|c|c|c|c|c|c|c|c|c|c|c|c|c|}
\hline Sample ID & & & & & & & CR & & & & & & \\
\hline $1 / 8 / 2017$ & $<\mathrm{MDL}$ & 0.030 & $<\mathrm{MDL}$ & $<\mathrm{MDL}$ & $<\mathrm{MDL}$ & $<\mathrm{MDL}$ & $<\mathrm{MDL}$ & 0.006 & 0.04 & $<\mathrm{MDL}$ & 0.18 & $<\mathrm{MDL}$ & 0.005 \\
\hline $4 / 10 / 2017$ & $<\mathrm{MDL}$ & 0.030 & $<\mathrm{MDL}$ & $<\mathrm{MDL}$ & $<\mathrm{MDL}$ & 0.01 & $<\mathrm{MDL}$ & 0.004 & 0.01 & $<\mathrm{MDL}$ & 0.15 & $<\mathrm{MDL}$ & 0.120 \\
\hline $7 / 12 / 2017$ & $<\mathrm{MDL}$ & 0.040 & $<\mathrm{MDL}$ & $<\mathrm{MDL}$ & $<\mathrm{MDL}$ & $<\mathrm{MDL}$ & $<\mathrm{MDL}$ & 0.007 & 0.02 & $<\mathrm{MDL}$ & 0.23 & $<\mathrm{MDL}$ & $<\mathrm{MDL}$ \\
\hline $10 / 25 / 2017$ & $<\mathrm{MDL}$ & 0.030 & $<\mathrm{MDL}$ & $<\mathrm{MDL}$ & $<\mathrm{MDL}$ & 0.02 & $<\mathrm{MDL}$ & 0.005 & 0.01 & $<\mathrm{MDL}$ & 0.19 & $<\mathrm{MDL}$ & 0.010 \\
\hline $1 / 1 / 2018$ & $<\mathrm{MDL}$ & 0.030 & $<\mathrm{MDL}$ & $<\mathrm{MDL}$ & $<\mathrm{MDL}$ & 0.03 & $<\mathrm{MDL}$ & 0.007 & 0.04 & $<\mathrm{MDL}$ & 0.18 & $<\mathrm{MDL}$ & 0.010 \\
\hline mean & $<\mathrm{MDL}$ & 0.032 & $<\mathrm{MDL}$ & $<\mathrm{MDL}$ & $<\mathrm{MDL}$ & 0.02 & $<\mathrm{MDL}$ & 0.006 & 0.02 & $<\mathrm{MDL}$ & 0.19 & $<\mathrm{MDL}$ & 0.036 \\
\hline
\end{tabular}

Notes:

-- No data; $\mathrm{n} / \mathrm{a}$ (not applicable)

ICP Dissolved by ICP (inductively coupled plasma)

MDL Method Detection Limit

NPDWRs National Primary Drinking Water Regulations - Max. Contaminant Level (MCL)

NSDWRs National Secondary Drinking Water Regulations - Secondary MCL

red Exceeds NPDWRs or NSDWRs 
Metalloids (As, B, Si) exhibited no discernible site-wide trends (Table 6). Arsenic values were below MDL (0.014 mg/L) for all samples except two: MW-6 on January 5, $2018(0.015 \mathrm{mg} / \mathrm{L})$, and MW-7 on July 10, $2017(0.017 \mathrm{mg} / \mathrm{L})$. These samples also exceeded the NPDWRs for arsenic $(0.01 \mathrm{mg} / \mathrm{L})$. The Cumberland River was below MDL for all sampling events. Boron values ranged from $<0.008-0.10 \mathrm{mg} / \mathrm{L}$ (mean $0.033 \mathrm{mg} / \mathrm{L}$ ) and silicon from $2.15-15.5 \mathrm{mg} / \mathrm{L}$ (mean $8.22 \mathrm{mg} / \mathrm{L}$ ). The exhibited boron values in the Cumberland River were similar to the onsite wells, while the silicon values were much lower (range 2.15-2.52 mg/L, mean $2.32 \mathrm{mg} / \mathrm{L}$ ).

Table 6. HLDTC Metalloids Analytical Results

\begin{tabular}{|c|c|c|c|}
\hline Analyte & As (ICP) & $\mathrm{B}(\mathrm{ICP})$ & $\mathrm{Si}(\mathrm{ICP})$ \\
\hline $\mathrm{MDL}(\mathrm{mg} / \mathrm{L})$ & 0.014 & 0.008 & 0.009 \\
\hline NPDWRs - MCL (mg/L) & 0.010 & $\mathrm{n} / \mathrm{a}$ & $\mathrm{n} / \mathrm{a}$ \\
\hline NSDWRs - SMCL (mg/L) & $\mathrm{n} / \mathrm{a}$ & $\mathrm{n} / \mathrm{a}$ & $\mathrm{n} / \mathrm{a}$ \\
\hline Sample ID & & MW-1 & \\
\hline $1 / 7 / 2017$ & $<\mathrm{MDL}$ & $<\mathrm{MDL}$ & 4.90 \\
\hline $4 / 7 / 2017$ & $<\mathrm{MDL}$ & $<\mathrm{MDL}$ & 3.85 \\
\hline $7 / 8 / 2017$ & $<\mathrm{MDL}$ & 0.015 & 4.69 \\
\hline $10 / 26 / 2017$ & $<\mathrm{MDL}$ & 0.020 & 4.43 \\
\hline $1 / 2 / 2018$ & $<\mathrm{MDL}$ & 0.008 & 3.77 \\
\hline mean & $<\mathrm{MDL}$ & 0.014 & 4.33 \\
\hline Sample ID & & MW-2 & \\
\hline $1 / 1 / 2017$ & -- & -- & -- \\
\hline $4 / 8 / 2017$ & $<\mathrm{MDL}$ & $<\mathrm{MDL}$ & 7.81 \\
\hline $7 / 8 / 2017$ & $<\mathrm{MDL}$ & 0.010 & 7.73 \\
\hline $10 / 26 / 2017$ & $<\mathrm{MDL}$ & $<\mathrm{MDL}$ & 8.40 \\
\hline $1 / 2 / 2018$ & $<\mathrm{MDL}$ & 0.010 & 8.93 \\
\hline mean & $<\mathrm{MDL}$ & 0.010 & 8.22 \\
\hline Sample ID & & MW-3R & \\
\hline $1 / 6 / 2017$ & $<\mathrm{MDL}$ & 0.010 & 11.5 \\
\hline $4 / 7 / 2017$ & $<\mathrm{MDL}$ & $<\mathrm{MDL}$ & 10.5 \\
\hline $7 / 8 / 2017$ & $<\mathrm{MDL}$ & 0.030 & 11.8 \\
\hline $10 / 26 / 2017$ & $<\mathrm{MDL}$ & 0.020 & 11.2 \\
\hline $1 / 2 / 2018$ & $<\mathrm{MDL}$ & 0.020 & 11.6 \\
\hline mean & $<\mathrm{MDL}$ & 0.023 & 11.3 \\
\hline
\end{tabular}


Table 6. HLDTC Metalloids Analytical Results (cont.)

\begin{tabular}{|c|c|c|c|}
\hline \multicolumn{4}{|l|}{ Sample ID } \\
\hline $1 / 6 / 2017$ & $<\mathrm{MDL}$ & 0.040 & 11.5 \\
\hline $4 / 7 / 2017$ & $<\mathrm{MDL}$ & 0.010 & 12.0 \\
\hline $7 / 9 / 2017$ & $<\mathrm{MDL}$ & 0.025 & 14.1 \\
\hline $10 / 28 / 2017$ & $<\mathrm{MDL}$ & 0.030 & 12.6 \\
\hline 10/28/2017 (duplicate) & $<\mathrm{MDL}$ & 0.030 & 12.3 \\
\hline $1 / 5 / 2018$ & $<\mathrm{MDL}$ & 0.030 & 12.6 \\
\hline mean & $<\mathrm{MDL}$ & 0.028 & 12.5 \\
\hline Sample ID & \multicolumn{2}{|c|}{ MW-5 } & \\
\hline $1 / 6 / 2017$ & $<\mathrm{MDL}$ & 0.050 & 12.9 \\
\hline $4 / 9 / 2017$ & $<\mathrm{MDL}$ & 0.020 & 10.2 \\
\hline $7 / 10 / 2017$ & $<\mathrm{MDL}$ & 0.080 & 14.7 \\
\hline $10 / 27 / 2017$ & $<\mathrm{MDL}$ & 0.030 & 12.8 \\
\hline $1 / 4 / 2018$ & $<\mathrm{MDL}$ & 0.050 & 13.2 \\
\hline mean & $<\mathrm{MDL}$ & 0.046 & 12.8 \\
\hline Sample ID & \multicolumn{2}{|c|}{ MW-6 } & \\
\hline $1 / 5 / 2017$ & $<\mathrm{MDL}$ & 0.040 & 8.47 \\
\hline $4 / 9 / 2017$ & $<\mathrm{MDL}$ & 0.030 & 7.83 \\
\hline 4/9/2017 (duplicate) & $<\mathrm{MDL}$ & 0.040 & 7.94 \\
\hline $7 / 10 / 2017$ & $<\mathrm{MDL}$ & 0.10 & 8.78 \\
\hline $10 / 27 / 2017$ & $<\mathrm{MDL}$ & 0.040 & 8.31 \\
\hline $1 / 5 / 2018$ & 0.015 & 0.070 & 8.83 \\
\hline 1/5/2018 (duplicate) & $<\mathrm{MDL}$ & 0.090 & 8.67 \\
\hline mean & 0.015 & 0.059 & 8.40 \\
\hline Sample ID & \multicolumn{2}{|c|}{ MW-7 } & \\
\hline $1 / 5 / 2017$ & $<\mathrm{MDL}$ & 0.030 & 6.36 \\
\hline $4 / 9 / 2017$ & $<\mathrm{MDL}$ & 0.030 & 5.90 \\
\hline $7 / 10 / 2017$ & 0.017 & 0.090 & 6.26 \\
\hline 7/10/2017 (duplicate) & 0.015 & 0.090 & 6.26 \\
\hline $10 / 27 / 2017$ & $<\mathrm{MDL}$ & 0.040 & 5.93 \\
\hline $1 / 5 / 2018$ & $<\mathrm{MDL}$ & 0.070 & 6.21 \\
\hline mean & 0.016 & 0.058 & 6.15 \\
\hline Sample ID & \multicolumn{2}{|c|}{ MW-8 } & \\
\hline $1 / 7 / 2017$ & $<\mathrm{MDL}$ & $<\mathrm{MDL}$ & 3.28 \\
\hline $4 / 8 / 2017$ & $<\mathrm{MDL}$ & $<\mathrm{MDL}$ & 3.23 \\
\hline $7 / 9 / 2017$ & $<\mathrm{MDL}$ & 0.010 & 3.85 \\
\hline $10 / 27 / 2017$ & $<\mathrm{MDL}$ & 0.020 & 3.76 \\
\hline $1 / 3 / 2018$ & $<\mathrm{MDL}$ & 0.020 & 4.27 \\
\hline mean & $<\mathrm{MDL}$ & 0.017 & 3.68 \\
\hline
\end{tabular}


Table 6. HLDTC Metalloids Analytical Results (cont.)



Notes:

-- No data; $\mathrm{n} / \mathrm{a}$ (not applicable)

ICP Dissolved by ICP (Inductively Coupled Plasma)

MDL Method Detection Limit

NPDWRs National Primary Drinking Water Regulations MCL

NSDWRs National Secondary Drinking Water Regulations - Secondary MCL

red Exceeds NPDWRs or NSDWRs 
Anion $\left(\mathrm{Br}^{-}, \mathrm{Cl}^{-}, \mathrm{F}^{-}, \mathrm{SO}_{4}{ }^{2-}\right)$ and nutrient $\left(\mathrm{NH}_{3}, \mathrm{NO}_{3}^{-}, \mathrm{P}, \mathrm{DOC}\right)$ results, along with bicarbonate $\left(\mathrm{HCO}_{3}^{-}\right)$and carbonate $\left(\mathrm{CO}_{3}^{2-}\right)$ values calculated via the USGS web-based alkalinity calculator (https://or.water.usgs.gov/alk), are presented in Table 7. There are no discernible site trends for these parameters. Bromide was $<$ MDL $(0.1 \mathrm{mg} / \mathrm{L})$ except for three samples: on January 5, 2017 (0.15 mg/L), a duplicate sample April 9, 2018 (0.165 $\mathrm{mg} / \mathrm{L})$, and October 27, $2018(0.1 \mathrm{mg} / \mathrm{L})$ at MW-6. Chloride values ranged from 1.00-7.58 $\mathrm{mg} / \mathrm{L}$ (mean $2.09 \mathrm{mg} / \mathrm{L}$ ). Fluoride was $<$ MDL (0.1 mg/L) in five wells (MW-1, MW-3R, MW-6, MW-8, MW-11); in other wells, values ranged from 0.11-0.27 mg/L (mean 0.15 $\mathrm{mg} / \mathrm{L})$. Sulfate was $<$ MDL ( $5 \mathrm{mg} / \mathrm{L}$ ) in MW-3R, MW-5, MW-7, and MW-10R; values in other wells ranged from $5.61-177 \mathrm{mg} / \mathrm{L}$ (mean $35.2 \mathrm{mg} / \mathrm{L}$ ). MW-2 exhibited unusually high sulfate values (39.4-177 mg/L, mean $92.85 \mathrm{mg} / \mathrm{L}$ ) and steadily decreased across sampling events, as was the case for major cations. Ammonia was $<\mathrm{MDL}(0.02 \mathrm{mg} / \mathrm{L})$ in MW-1, MW-10R, and MW-11 (with the exception of the January 8, 2017 sample). Values in other wells ranged from $0.02-1.25 \mathrm{mg} / \mathrm{L}$ (mean $0.32 \mathrm{mg} / \mathrm{L}$ ). Nitrate ranged from 0.10 $3.07 \mathrm{mg} / \mathrm{L}$ (mean $1.01 \mathrm{mg} / \mathrm{L}$ ). Phosphorus was $<$ MDL $(0.009 \mathrm{mg} / \mathrm{L}$ ) in MW-1 and MW10R; other values ranged from $0.009-0.80 \mathrm{mg} / \mathrm{L}$ (mean $0.27 \mathrm{mg} / \mathrm{L}$ ). DOC ranged from < MDL $(0.3 \mathrm{mg} / \mathrm{L}$ ) to $17.23 \mathrm{mg} / \mathrm{L}$ (mean $1.45 \mathrm{mg} / \mathrm{L}$ ) in wells and $<0.30$ to $4.28 \mathrm{mg} / \mathrm{L}$ in the river. Bicarbonate ranged from $0.3-159.5 \mathrm{mg} / \mathrm{L}$ (mean $59.6 \mathrm{mg} / \mathrm{L}$ ). Except for MW-2 on July 8, 2017 (0.1 mg/L), MW-6 on January 5, 2017 (0.1 mg/L), and the Cumberland River on January 8, $2017(0.1 \mathrm{mg} / \mathrm{L})$ and July 12, $2017(0.8 \mathrm{mg} / \mathrm{L})$, all calculated carbonate values were $<0.1 \mathrm{mg} / \mathrm{L}$. The Cumberland River anion and nutrient results are well within the onsite well ranges but exhibit slightly higher mean values. The laboratory analysis reports 
for data listed in Tables 3-7 (apart from bicarbonate and carbonate) are included in the appendices as KGS Quarter No. Analytical Report. 
Table 7. HLDTC Anion and Nutrient Analytical Results

\begin{tabular}{|c|c|c|c|c|c|c|c|c|c|c|c|}
\hline Analyte & $\mathrm{Br}$ & $\mathrm{Cl}$ & $\mathrm{F}$ & $\mathrm{NH}_{3}$ & $\mathrm{NO}_{3}$ & $\begin{array}{l}\mathrm{P} \\
(\mathrm{ICP})\end{array}$ & $\begin{array}{l}\mathrm{S} \\
(\mathrm{ICP})\end{array}$ & $\mathrm{SO}_{4}$ & $\mathrm{TOC}$ & $\mathrm{HCO}_{3}$ & $\mathrm{CO}_{3}$ \\
\hline $\operatorname{MDL}(\mathrm{mg} / \mathrm{L})$ & 0.10 & 1.00 & 0.10 & 0.02 & 0.10 & 0.009 & 0.014 & 5.0 & 0.30 & $\mathrm{n} / \mathrm{a}$ & $\mathrm{n} / \mathrm{a}$ \\
\hline NPDWRs - & & & & & & & & & & & \\
\hline $\operatorname{MCL}(\mathrm{mg} / \mathrm{L})$ & $\mathrm{n} / \mathrm{a}$ & $\mathrm{n} / \mathrm{a}$ & 4.00 & $\mathrm{n} / \mathrm{a}$ & 10.00 & $\mathrm{n} / \mathrm{a}$ & $\mathrm{n} / \mathrm{a}$ & $\mathrm{n} / \mathrm{a}$ & $\mathrm{n} / \mathrm{a}$ & $\mathrm{n} / \mathrm{a}$ & $\mathrm{n} / \mathrm{a}$ \\
\hline NSDWRs - & & & & & & & & & & & \\
\hline SMCL (mg/L) & $\mathrm{n} / \mathrm{a}$ & 250 & 2.00 & $\mathrm{n} / \mathrm{a}$ & $\mathrm{n} / \mathrm{a}$ & $\mathrm{n} / \mathrm{a}$ & $\mathrm{n} / \mathrm{a}$ & 250 & $\mathrm{n} / \mathrm{a}$ & $\mathrm{n} / \mathrm{a}$ & $\mathrm{n} / \mathrm{a}$ \\
\hline Sample ID & & & & & & MW-1 & & & & & \\
\hline $1 / 7 / 2017$ & $<\mathrm{MDL}$ & 1.11 & $<\mathrm{MDL}$ & $<\mathrm{MDL}$ & 0.95 & $<\mathrm{MDL}$ & 0.95 & $<\mathrm{MDL}$ & 1.36 & 6.8 & $<0.1$ \\
\hline $4 / 7 / 2017$ & $<\mathrm{MDL}$ & 1.37 & $<\mathrm{MDL}$ & $<\mathrm{MDL}$ & 1.66 & $<\mathrm{MDL}$ & 2.23 & 7.33 & 0.44 & 5 & $<0.1$ \\
\hline $7 / 8 / 2017$ & $<\mathrm{MDL}$ & 1.43 & $<\mathrm{MDL}$ & $<\mathrm{MDL}$ & 3.07 & $<\mathrm{MDL}$ & $<\mathrm{MDL}$ & $<\mathrm{MDL}$ & $<\mathrm{MDL}$ & 3.3 & $<0.1$ \\
\hline $10 / 26 / 2017$ & $<\mathrm{MDL}$ & 1.48 & $<\mathrm{MDL}$ & $<\mathrm{MDL}$ & 2.94 & $<\mathrm{MDL}$ & 0.26 & $<\mathrm{MDL}$ & 0.39 & 4.6 & $<0.1$ \\
\hline $1 / 2 / 2018$ & $<\mathrm{MDL}$ & 1.42 & $<\mathrm{MDL}$ & $<\mathrm{MDL}$ & 1.53 & $<\mathrm{MDL}$ & 1.86 & 6.63 & 0.32 & 8 & $<0.1$ \\
\hline mean & $<\mathrm{MDL}$ & 1.36 & $<\mathrm{MDL}$ & $<\mathrm{MDL}$ & 2.03 & $<\mathrm{MDL}$ & 1.33 & 6.98 & 0.63 & 5.54 & $<0.1$ \\
\hline Sample ID & & & & & & MW-2 & & & & & \\
\hline $1 / 1 / 2017$ & -- & -- & -- & -- & -- & -- & -- & -- & -- & -- & -- \\
\hline $4 / 8 / 2017$ & $<\mathrm{MDL}$ & 7.58 & $<\mathrm{MDL}$ & 0.03 & $<\mathrm{MDL}$ & $<\mathrm{MDL}$ & 50.6 & 177.00 & 1.27 & 159.5 & $<0.1$ \\
\hline $7 / 8 / 2017$ & $<\mathrm{MDL}$ & 2.78 & $<\mathrm{MDL}$ & 0.03 & 0.10 & $<\mathrm{MDL}$ & 2.05 & 87.60 & $<\mathrm{MDL}$ & 96.5 & 0.1 \\
\hline $10 / 26 / 2017$ & $<\mathrm{MDL}$ & 1.98 & 0.26 & $<\mathrm{MDL}$ & 0.41 & 0.01 & 21.9 & 67.40 & 0.87 & 62.9 & $<0.1$ \\
\hline $1 / 2 / 2018$ & $<\mathrm{MDL}$ & 1.36 & $<\mathrm{MDL}$ & $<\mathrm{MDL}$ & 0.20 & 0.01 & 16.5 & 39.40 & 0.42 & 49.3 & $<0.1$ \\
\hline mean & $<\mathrm{MDL}$ & 3.43 & 0.26 & 0.03 & 0.24 & 0.01 & 22.76 & 92.85 & 0.85 & 92.05 & $<0.1$ \\
\hline Sample ID & & & & & & MW-3R & & & & & \\
\hline $1 / 6 / 2017$ & $<\mathrm{MDL}$ & 1.94 & $<\mathrm{MDL}$ & 0.09 & $<\mathrm{MDL}$ & 0.09 & 0.57 & $<\mathrm{MDL}$ & 2.04 & 48.7 & $<0.1$ \\
\hline $4 / 7 / 2017$ & $<\mathrm{MDL}$ & 2.00 & $<\mathrm{MDL}$ & 0.08 & $<\mathrm{MDL}$ & 0.23 & 0.92 & $<\mathrm{MDL}$ & 0.31 & 58.6 & $<0.1$ \\
\hline $7 / 8 / 2017$ & $<\mathrm{MDL}$ & 2.06 & $<\mathrm{MDL}$ & 0.07 & $<\mathrm{MDL}$ & 0.26 & $<\mathrm{MDL}$ & $<\mathrm{MDL}$ & $<\mathrm{MDL}$ & 41.3 & $<0.1$ \\
\hline $10 / 26 / 2017$ & $<\mathrm{MDL}$ & 2.10 & $<\mathrm{MDL}$ & $<\mathrm{MDL}$ & $<\mathrm{MDL}$ & 0.28 & 0.99 & $<\mathrm{MDL}$ & 0.70 & 29.6 & $<0.1$ \\
\hline $1 / 2 / 2018$ & $<\mathrm{MDL}$ & 2.04 & $<\mathrm{MDL}$ & $<\mathrm{MDL}$ & $<\mathrm{MDL}$ & 0.27 & 0.89 & $<\mathrm{MDL}$ & 0.11 & 47.5 & $<0.1$ \\
\hline mean & $<\mathrm{MDL}$ & 2.03 & $<\mathrm{MDL}$ & 0.08 & $<\mathrm{MDL}$ & 0.23 & 0.84 & $<\mathrm{MDL}$ & 0.79 & 45.14 & $<0.1$ \\
\hline
\end{tabular}


Table 7. HLDTC Anion and Nutrient Analytical Results (cont.)

\begin{tabular}{|c|c|c|c|c|c|c|c|c|c|c|c|}
\hline \multicolumn{6}{|l|}{ Sample ID } & \multicolumn{6}{|l|}{ MW-4 } \\
\hline $1 / 6 / 2017$ & $<\mathrm{MDL}$ & 3.97 & $<\mathrm{MDL}$ & 0.18 & $<\mathrm{MDL}$ & $<\mathrm{MDL}$ & 0.52 & $<\mathrm{MDL}$ & 6.58 & 94.3 & $<0.1$ \\
\hline 4/7/2017 & $<\mathrm{MDL}$ & 3.71 & $<\mathrm{MDL}$ & 0.10 & $<\mathrm{MDL}$ & $<\mathrm{MDL}$ & 12.4 & 39.10 & 1.36 & 92.4 & $<0.1$ \\
\hline $7 / 9 / 2017$ & $<\mathrm{MDL}$ & 2.80 & $<\mathrm{MDL}$ & 0.12 & $<\mathrm{MDL}$ & $<\mathrm{MDL}$ & 1.47 & 32.40 & $<\mathrm{MDL}$ & 86.2 & $<0.1$ \\
\hline $10 / 28 / 2017$ & $<\mathrm{MDL}$ & 2.53 & 0.16 & $<\mathrm{MDL}$ & 0.15 & 0.01 & 13.2 & 41.70 & 0.55 & 70.3 & $<0.1$ \\
\hline \multicolumn{12}{|l|}{$10 / 28 / 2017$} \\
\hline (duplicate) & $<\mathrm{MDL}$ & 2.52 & 0.14 & $<\mathrm{MDL}$ & 0.15 & 0.02 & 13.0 & 42.70 & 0.45 & 69.6 & $<0.1$ \\
\hline $1 / 5 / 2018$ & $<\mathrm{MDL}$ & 2.42 & 0.11 & $<\mathrm{MDL}$ & 0.16 & 0.01 & 11.5 & 33.20 & 0.36 & 63.5 & $<0.1$ \\
\hline mean & $<\mathrm{MDL}$ & 2.99 & 0.14 & 0.13 & 0.15 & 0.01 & 8.68 & 37.82 & 1.86 & 79.4 & $<0.1$ \\
\hline Sample ID & \multicolumn{11}{|c|}{ MW-5 } \\
\hline $1 / 6 / 2017$ & $<\mathrm{MDL}$ & 4.44 & $<\mathrm{MDL}$ & 0.21 & $<\mathrm{MDL}$ & 0.10 & 0.41 & $<\mathrm{MDL}$ & 17.23 & 66.6 & $<0.1$ \\
\hline $4 / 9 / 2017$ & $<\mathrm{MDL}$ & 1.94 & $<\mathrm{MDL}$ & 0.26 & $<\mathrm{MDL}$ & 0.51 & 0.30 & $<\mathrm{MDL}$ & 1.32 & 83.2 & $<0.1$ \\
\hline $7 / 10 / 2017$ & $<\mathrm{MDL}$ & 2.25 & $<\mathrm{MDL}$ & 0.11 & $<\mathrm{MDL}$ & 0.09 & 3.36 & $<\mathrm{MDL}$ & $<\mathrm{MDL}$ & 63.5 & $<0.1$ \\
\hline $10 / 27 / 2017$ & $<\mathrm{MDL}$ & 2.37 & 0.12 & $<\mathrm{MDL}$ & $<\mathrm{MDL}$ & 0.20 & 0.10 & $<\mathrm{MDL}$ & 1.22 & 74 & $<0.1$ \\
\hline $1 / 4 / 2018$ & $<\mathrm{MDL}$ & 2.34 & 0.12 & $<\mathrm{MDL}$ & $<\mathrm{MDL}$ & 0.25 & 0.23 & $<\mathrm{MDL}$ & 0.90 & 82.6 & $<0.1$ \\
\hline mean & $<\mathrm{MDL}$ & 2.67 & 0.12 & 0.19 & $<\mathrm{MDL}$ & 0.23 & 0.88 & $<\mathrm{MDL}$ & 5.17 & 74.0 & $<0.1$ \\
\hline Sample ID & \multicolumn{11}{|c|}{ MW-6 } \\
\hline $1 / 5 / 2017$ & 0.15 & 1.47 & $<\mathrm{MDL}$ & 0.67 & $<\mathrm{MDL}$ & 0.54 & 2.07 & 7.39 & 1.53 & 138.5 & 0.1 \\
\hline $4 / 9 / 2017$ & $<\mathrm{MDL}$ & 1.28 & $<\mathrm{MDL}$ & 0.56 & $<\mathrm{MDL}$ & 0.61 & 1.69 & 5.99 & 1.10 & 149.7 & $<0.1$ \\
\hline $4 / 9 / 2017$ & & & & & & & & & & & \\
\hline (duplicate) & 0.16 & 1.25 & $<\mathrm{MDL}$ & 0.54 & $<\mathrm{MDL}$ & 0.65 & 1.63 & 5.88 & 1.08 & 131.2 & $<0.1$ \\
\hline $7 / 10 / 2017$ & $<\mathrm{MDL}$ & 1.48 & $<\mathrm{MDL}$ & 0.59 & $<\mathrm{MDL}$ & 0.69 & 0.93 & $<\mathrm{MDL}$ & $<\mathrm{MDL}$ & 94.9 & $<0.1$ \\
\hline $10 / 27 / 2017$ & 0.10 & 1.83 & $<\mathrm{MDL}$ & $<\mathrm{MDL}$ & 0.11 & 0.67 & 1.63 & 6.31 & 0.67 & 101.7 & $<0.1$ \\
\hline $1 / 5 / 2018$ & $<\mathrm{MDL}$ & 1.43 & $<\mathrm{MDL}$ & $<\mathrm{MDL}$ & $<\mathrm{MDL}$ & 0.80 & 0.85 & $<\mathrm{MDL}$ & 0.74 & 124.5 & $<0.1$ \\
\hline $1 / 5 / 2018$ & & & & & & & & & & & \\
\hline (duplicate) & $<\mathrm{MDL}$ & 1.43 & $<\mathrm{MDL}$ & $<\mathrm{MDL}$ & $<\mathrm{MDL}$ & 0.79 & 0.94 & $<\mathrm{MDL}$ & 0.49 & 107.8 & $<0.1$ \\
\hline mean & 0.14 & 1.45 & $<\mathrm{MDL}$ & 0.59 & 0.11 & 0.68 & 1.39 & 6.39 & 0.94 & 121.2 & $<0.1$ \\
\hline
\end{tabular}


Table 7. HLDTC Anion and Nutrient Analytical Results (cont.)

\begin{tabular}{|c|c|c|c|c|c|c|c|c|c|c|c|}
\hline Sample ID & & & & & & MW-7 & & & & & \\
\hline $1 / 5 / 2017$ & $<\mathrm{MDL}$ & $<\mathrm{MDL}$ & $<\mathrm{MDL}$ & 1.20 & $<\mathrm{MDL}$ & 0.50 & 0.43 & $<\mathrm{MDL}$ & 0.71 & 118.9 & $<0.1$ \\
\hline $4 / 9 / 2017$ & $<\mathrm{MDL}$ & $<\mathrm{MDL}$ & 0.11 & 1.25 & $<\mathrm{MDL}$ & 0.60 & 0.40 & $<\mathrm{MDL}$ & 2.02 & 75.8 & $<0.1$ \\
\hline $7 / 10 / 2017$ & $<\mathrm{MDL}$ & $<\mathrm{MDL}$ & $<\mathrm{MDL}$ & 1.15 & $<\mathrm{MDL}$ & 0.59 & 1.31 & $<\mathrm{MDL}$ & $<\mathrm{MDL}$ & 69.7 & $<0.1$ \\
\hline $7 / 10 / 2017$ & & & & & & & & & & & \\
\hline (duplicate) & $\mathrm{MDL}$ & $<\mathrm{MDL}$ & $<\mathrm{MDL}$ & 1.09 & $<\mathrm{MDL}$ & 0.61 & 1.20 & $<\mathrm{MDL}$ & $<\mathrm{MDL}$ & 61.6 & $<0.1$ \\
\hline $10 / 27 / 2017$ & $<\mathrm{MDL}$ & 1.03 & $<\mathrm{MDL}$ & $<\mathrm{MDL}$ & $<\mathrm{MDL}$ & 0.60 & 0.53 & $<\mathrm{MDL}$ & 0.77 & 67.2 & $<0.1$ \\
\hline $1 / 5 / 2018$ & $<\mathrm{MDL}$ & $<\mathrm{MDL}$ & 0.12 & $<\mathrm{MDL}$ & $<\mathrm{MDL}$ & 0.63 & 0.61 & $<\mathrm{MDL}$ & 1.19 & 86.9 & $<0.1$ \\
\hline mean & $<\mathrm{MDL}$ & 1.03 & 0.12 & 1.17 & $<\mathrm{MDL}$ & 0.59 & 0.75 & $<\mathrm{MDL}$ & 1.17 & 80.0 & $<0.1$ \\
\hline ole ID & & & & & & MW-8 & & & & & \\
\hline $1 / 7 / 2017$ & $<\mathrm{MDL}$ & $<\mathrm{MDL}$ & $<\mathrm{MDL}$ & 0.02 & $<\mathrm{MDL}$ & $<\mathrm{MDL}$ & 1.71 & 6.41 & 1.61 & 4.9 & $<0.1$ \\
\hline $4 / 8 / 2017$ & $<\mathrm{MDL}$ & $<\mathrm{MDL}$ & $<\mathrm{MDL}$ & 0.02 & $<\mathrm{MDL}$ & $<\mathrm{MDL}$ & 2.00 & 6.64 & 0.64 & 41.6 & $<0.1$ \\
\hline $7 / 9 / 2017$ & $<\mathrm{MDL}$ & 1.08 & $<\mathrm{MDL}$ & 0.02 & $<\mathrm{MDL}$ & $<\mathrm{MDL}$ & 0.39 & 7.34 & $<\mathrm{MDL}$ & 4.5 & $<0.1$ \\
\hline $10 / 27 / 2017$ & $<\mathrm{MDL}$ & 1.09 & $<\mathrm{MDL}$ & $<\mathrm{MDL}$ & $<\mathrm{MDL}$ & 0.01 & 2.15 & 7.67 & 0.66 & 7.6 & $<0.1$ \\
\hline $1 / 3 / 2018$ & $<\mathrm{MDL}$ & 1.19 & $<\mathrm{MDL}$ & $<\mathrm{MDL}$ & $<\mathrm{MDL}$ & 0.01 & 2.76 & 9.54 & 0.37 & 6.5 & $<0.1$ \\
\hline mean & $<\mathrm{MDL}$ & 1.12 & $<\mathrm{MDL}$ & 0.02 & $<\mathrm{MDL}$ & 0.01 & 1.80 & 7.52 & 0.52 & 13.0 & $<0.1$ \\
\hline ample ID & & & & & & MW-9R & & & & & \\
\hline $1 / 7 / 2017$ & $<\mathrm{MDL}$ & 3.19 & $<\mathrm{MDL}$ & 0.58 & 2.08 & $<\mathrm{MDL}$ & 1.75 & 6.46 & 2.22 & 106.6 & $<0.1$ \\
\hline $4 / 9 / 2017$ & $<\mathrm{MDL}$ & 2.64 & $<\mathrm{MDL}$ & 0.17 & 2.18 & $<\mathrm{MDL}$ & 1.82 & 6.40 & 0.69 & 85.6 & $<0.1$ \\
\hline $7 / 8 / 2017$ & $<\mathrm{MDL}$ & 2.16 & 0.15 & 0.25 & 1.66 & $<\mathrm{MDL}$ & $<\mathrm{MDL}$ & 6.44 & $<\mathrm{MDL}$ & 99.2 & $<0.1$ \\
\hline $10 / 26 / 2017$ & $<\mathrm{MDL}$ & 2.27 & 0.17 & $<\mathrm{MDL}$ & 0.98 & 0.01 & 1.68 & 6.44 & 0.92 & 70.9 & $<0.1$ \\
\hline $1 / 4 / 2018$ & $<\mathrm{MDL}$ & 1.86 & 0.14 & $<\mathrm{MDL}$ & 0.18 & $<\mathrm{MDL}$ & 1.71 & 5.61 & 0.21 & 83.8 & $<0.1$ \\
\hline mean & $<\mathrm{MDL}$ & 2.42 & 0.15 & 0.33 & 1.42 & 0.01 & 1.74 & 6.27 & 1.01 & 89.2 & $<0.1$ \\
\hline
\end{tabular}


Table 7. HLDTC Anion and Nutrient Analytical Results (cont.)

\begin{tabular}{|c|c|c|c|c|c|c|c|c|c|c|c|}
\hline Sample ID & & & & & & IW-10R & & & & & \\
\hline $1 / 8 / 2017$ & $<\mathrm{MDL}$ & 1.19 & $<\mathrm{MDL}$ & $<\mathrm{MDL}$ & 0.60 & $<\mathrm{MDL}$ & 0.24 & $<\mathrm{MDL}$ & 1.02 & 11.1 & $<0.1$ \\
\hline $1 / 8 / 2017$ & & & & & & & & & & & \\
\hline (duplicate) & $<\mathrm{MDL}$ & 1.20 & $<\mathrm{MDL}$ & $<\mathrm{MDL}$ & 0.59 & $<\mathrm{MDL}$ & 0.20 & $<\mathrm{MDL}$ & 1.54 & 21 & $<0.1$ \\
\hline $4 / 8 / 2017$ & $<\mathrm{MDL}$ & 1.42 & $<\mathrm{MDL}$ & $<\mathrm{MDL}$ & 0.69 & $<\mathrm{MDL}$ & 0.21 & $<\mathrm{MDL}$ & 0.39 & 7.2 & $<0.1$ \\
\hline $7 / 9 / 2017$ & $<\mathrm{MDL}$ & 1.33 & $<\mathrm{MDL}$ & $<\mathrm{MDL}$ & 0.76 & $<\mathrm{MDL}$ & $<\mathrm{MDL}$ & $<\mathrm{MDL}$ & FMDL & 7 & $<0.1$ \\
\hline $10 / 27 / 2017$ & $<\mathrm{MDL}$ & 1.37 & $<\mathrm{MDL}$ & $<\mathrm{MDL}$ & 0.88 & $<\mathrm{MDL}$ & 0.44 & $<\mathrm{MDL}$ & 0.35 & 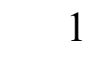 & $<0.1$ \\
\hline $1 / 1 / 2018$ & $<\mathrm{MDL}$ & 1.24 & $<\mathrm{MDL}$ & $<\mathrm{MDL}$ & 0.76 & $<\mathrm{MDL}$ & 0.70 & $<\mathrm{MDL}$ & 0.25 & 9 & $<0.1$ \\
\hline mean & $<\mathrm{MDL}$ & 1.29 & $<\mathrm{MDL}$ & $<\mathrm{MDL}$ & 0.71 & $<\mathrm{MDL}$ & 0.34 & $<\mathrm{MDL}$ & 0.71 & 9.4 & $<0.1$ \\
\hline Sample ID & & & & & & MW-11 & & & & & \\
\hline $1 / 8 / 2017$ & $<\mathrm{MDL}$ & 2.25 & $<\mathrm{MDL}$ & 0.02 & 0.45 & 0.02 & 7.71 & 24.1 & 1.21 & 21.4 & $<0.1$ \\
\hline $4 / 10 / 2017$ & $<\mathrm{MDL}$ & 3.54 & $<\mathrm{MDL}$ & $<\mathrm{MDL}$ & 0.38 & $<\mathrm{MDL}$ & 14.1 & 43.4 & 1.43 & 18.7 & $<0.1$ \\
\hline $7 / 9 / 2017$ & $<\mathrm{MDL}$ & 3.29 & $<\mathrm{MDL}$ & $<\mathrm{MDL}$ & $<\mathrm{MDL}$ & $<\mathrm{MDL}$ & 0.48 & 46.2 & $<\mathrm{MDL}$ & 7.7 & $<0.1$ \\
\hline $10 / 28 / 2017$ & $<\mathrm{MDL}$ & 3.69 & $<\mathrm{MDL}$ & $<\mathrm{MDL}$ & 0.40 & 0.01 & 17.0 & 55.4 & 0.78 & 11.8 & $<0.1$ \\
\hline $1 / 3 / 2018$ & $<\mathrm{MDL}$ & 3.31 & $<\mathrm{MDL}$ & $<\mathrm{MDL}$ & 0.85 & 0.01 & 16.3 & 33.4 & 0.62 & 12.2 & $<0.1$ \\
\hline mean & $<\mathrm{MDL}$ & 3.22 & $<\mathrm{MDL}$ & 0.02 & 0.52 & 0.01 & 11.1 & 40.5 & 1.01 & 14.4 & $<0.1$ \\
\hline Sample ID & & & & & & TF & & & & & \\
\hline $1 / 1 / 2017$ & -- & -- & -- & -- & -- & -- & -- & -- & -- & -- & . \\
\hline $4 / 10 / 2017$ & $<\mathrm{MDL}$ & 1.28 & 0.27 & 0.02 & 41 & 0.04 & 0.36 & $<\mathrm{MDL}$ & $<\mathrm{MDL}$ & 60 & $<0.1$ \\
\hline $7 / 10 / 2017$ & $<\mathrm{MDL}$ & 1.13 & 0.12 & $<\mathrm{MDL}$ & 0.89 & 0.05 & $<\mathrm{MDL}$ & $<\mathrm{MDL}$ & $<\mathrm{MDL}$ & 48.1 & $<0.1$ \\
\hline $10 / 28 / 2017$ & $<\mathrm{MDL}$ & 1.23 & 0.11 & $<\mathrm{MDL}$ & 0.96 & 0.05 & 0.26 & $<\mathrm{MDL}$ & $<\mathrm{MDL}$ & 67.8 & $<0.1$ \\
\hline $1 / 4 / 2018$ & $<\mathrm{MDL}$ & 1.17 & $<\mathrm{MDL}$ & $<\mathrm{MDL}$ & 0.77 & 0.07 & 0.25 & $<\mathrm{MDL}$ & $<\mathrm{MDL}$ & 68.5 & $<0.1$ \\
\hline mean & $<\mathrm{MDL}$ & 1.20 & 0.17 & 0.02 & 1.01 & 0.05 & 0.29 & $<\mathrm{MDL}$ & $<\mathrm{MDL}$ & 61.1 & $<0.1$ \\
\hline
\end{tabular}


Table 7. HLDTC Anion and Nutrient Analytical Results (cont.)

\begin{tabular}{rlrlrrrrrrrr}
\hline Sample ID & & \multicolumn{10}{c}{ CR } \\
$1 / 8 / 2017$ & $<$ MDL & 5.08 & $<$ MDL & 0.02 & 1.68 & $<$ MDL & 25.7 & 80.3 & 2.20 & 87.1 & 0.2 \\
4/10/2017 & $<$ MDL & 2.84 & $<$ MDL & 0.02 & 1.24 & $<$ MDL & 23.1 & 68.1 & 3.01 & 81.5 & $<0.1$ \\
$7 / 12 / 2017$ & $<$ MDL & 3.75 & 0.16 & 0.02 & 0.92 & 0.02 & 36.5 & 89.6 & $<$ MDL & 132.1 & 0.8 \\
$10 / 25 / 2017$ & $<$ MDL & 4.64 & 0.15 & $<$ MDL & 2.09 & 0.02 & 21.1 & 67.3 & 4.28 & 0.3 & $<0.1$ \\
$1 / 1 / 2018$ & $<$ MDL & 3.75 & 0.19 & $<$ MDL & 1.43 & 0.02 & 26.3 & 56.3 & 1.30 & 70.6 & $<0.1$ \\
mean & $<$ MDL & 4.01 & 0.17 & 0.02 & 1.47 & 0.02 & 26.5 & 72.3 & 2.70 & 74.3 & 0.26 \\
\hline
\end{tabular}

Notes:

-- No data; n/a (not applicable)

ICP Dissolved by ICP (Inductively Coupled Plasma)

MDL Method Detection Limit

NPDWRs National Primary Drinking Water Regulations - MCL

NSDWRs National Secondary Drinking Water Regulations - Secondary MCL

italicized Suspect titration inflection point 
Comparable water quality parameter and chemical analytical data are limited within the literature. Studies conducted within $100 \mathrm{~km}$ of HLDTC have focused on determining local or regional groundwater flow paths. Water chemistry analysis were, for the most part, limited to field parameters $(\mathrm{pH}$, temperature, $\mathrm{SC})$ and major cations $(\mathrm{Ca}, \mathrm{Mg}, \mathrm{K}, \mathrm{Na})$ used to determine hydrochemical facies, as discussed later. The wells and/or piezometers were completed within bedrock units (the upper and middle Breathitt Fm.) that do not exist at HLDTC. Furthermore, the wells in other studies were constructed to depths ( $\sim 45-150 \mathrm{~m}$ bls) that exceed those at HLDTC (monitoring wells range 3.6-13.6 m bls; tree farm well $36.6 \mathrm{~m}$ bls) and few are located in valley bottoms or within alluvium (Wunsch, 1993; Price et al., 1962; Kipp and Dinger, 1991; Minns, 1993). Therefore, the water-quality parameter and solute analysis comparison focused on data provided for individual wells that were located within the Breathitt Fm. (ideally lower units) or, based upon available core logs, completed within weathered sandstones, siltstones or shale. Based upon observations and cuttings collected during HLDTC monitoring well installation, these rock types resemble those in which the site wells terminate. Core logs that noted carbonaceous formations or materials were excluded because TIC and coal were not detected in the core samples analyzed in this study, as discussed later.

The wells studied by Wunsch (1993) were not comparable to those at HLDTC (completed in upper Breathitt or Lee Formations, core logs noted carbonaceous materials, total depth range 20.4 to $128 \mathrm{~m}$ bls, and placement was not within alluvium). However, Wunsch (1993) reported precipitation chemistry data collected at the Robinson Forest monitoring station for the 1-yr study duration. The mean values of the reported precipitation parameters (conductivity $15.4 \mu \mathrm{S} / \mathrm{cm} ; \mathrm{SO}^{2-} 1.8 \mathrm{mg} / \mathrm{L} ; \mathrm{Mg} 0.06 \mathrm{mg} / \mathrm{L} ; \mathrm{Ca} 0.55$ 
$\mathrm{mg} / \mathrm{L} ; \mathrm{K} 0.05 \mathrm{mg} / \mathrm{L} ; \mathrm{Na} 0.15 \mathrm{mg} / \mathrm{L}$; alkalinity as $\mathrm{HCO}_{3}{ }^{-} 20.5 \mathrm{mg} / \mathrm{L} ; \mathrm{pH} 4.1 ; \mathrm{PO}_{4}{ }^{3-} 0.01$ $\mathrm{mg} / \mathrm{L}$ ) tend to fall within the low range of the HLDTC water chemistry data. The similarity between the precipitation and groundwater chemistry data suggests relatively rapid infiltration within the HLDTC alluvium, where precipitation is the dominant source of groundwater recharge (McCoy et al., 2015). Price et al. (1962) included a summary of dissolved constituents and other properties of water from wells drilled in Appalachian Kentucky. Well construction details were not provided, but location descriptions were given based on physiographic region and geology. The descriptions resembling HLDTC were Cumberland Plateau, Breathitt Fm. (8 samples); Kanawha section, Breathitt Fm. (41 samples); and Kanawha section, alluvium (tributary valley to the Ohio River) (1 sample), where the latter is the closest representation. Median constituent results were given for Fe, $\mathrm{HCO}_{3}{ }^{-}, \mathrm{SO}_{4}{ }^{2-}, \mathrm{Cl}^{-}, \mathrm{F}^{-}, \mathrm{NO}_{3}{ }^{-}, \mathrm{SC}$, and $\mathrm{pH}$. The results are similar to mean values at HLDTC. Minns (1993) installed three sets of nested piezometers, one of which was completed in the upper Breathitt Fm. and located in a valley bottom $\sim 65 \mathrm{~m}$ from the Edd Fork tributary. The core logs describe the rock as heavily weathered sandstone lacking carbonaceous material. Two piezometers within the nest were completed to similar depths as HLDTC's monitoring wells, 5.2 and $7.3 \mathrm{~m}$ bls. Water chemistry was only sampled once. The results, which Minns (1993) described as "low in dissolved constituents", were compared to the means and ranges at HLDTC and found to be in good agreement with the exception of iron (Minns: 0.112 and $0.648 \mathrm{mg} / \mathrm{L}$; HDLTC: $0.002-39.4 \mathrm{mg} / \mathrm{L}$, mean $9.32 \mathrm{mg} / \mathrm{L}$ ). Overall, at the regional scale, the water chemistry at HLDTC is broadly similar to that reported in other studies for wells in comparable hydrogeologic settings. 
Piper diagrams (Figure 13) show that major-ion hydrochemical facies were primarily mixed cation- $\mathrm{HCO}_{3}$ for all five quarters from January 2017 to January 2018, although wells MW-2 and MW-11 were consistently Na-SO 4 type. As noted above, results for MW-2 are suspect and suggest that the well was not properly developed. From a limited number of water samples in eastern Kentucky, Kipp et al. (1983) observed $\mathrm{Na}-\mathrm{HCO}_{3}$ hydrochemical facies below local drainage and $\mathrm{Ca}-\mathrm{HCO}_{3}$ or mixed-cation- $\mathrm{HCO}_{3}$ from hillside samples. Wunsch (1993) refined the facies to be $\mathrm{Ca}-\mathrm{Mg}-\mathrm{HCO}_{3}$ for water derived from coal seams, $\mathrm{Ca}-\mathrm{HCO}_{3}$ and $\mathrm{Mg}-\mathrm{SO}_{4}$ from within fracture zones, and $\mathrm{Na}-\mathrm{HCO}_{3}$ from ridge interiors. Generally, wells bottoming below major streams in eastern Kentucky tend to have $\mathrm{Na}-\mathrm{HCO}_{3}$ or $\mathrm{Na}-\mathrm{Cl}$ hydrochemical facies, while those along hillsides are $\mathrm{Ca}-\mathrm{HCO}_{3}$ or $\mathrm{Ca}_{-} \mathrm{SO}_{4}$ (Wunsch, 1993).

The monitoring wells in this study were near the base of the ridge (along hillsides), near the river (below local drainage), or in-between along anticipated flow paths. None were drilled within ridge interiors. Based upon drilling logs and collected soil cores, the wells were not placed within coal seams nor, following a cursory review of Google Earth images, within lineaments associated with fractures (Kipp and Dinger, 1991). Analysis of higher-resolution LiDAR aerial images was beyond the scope of this project. Based on monitoring well placement, the mixed cation- $\mathrm{HCO}_{3}$ hydrochemical facies in this study agrees with previous studies, while $\mathrm{Na}_{-} \mathrm{SO}_{4}$ does not. A simplified conceptual flow model for eastern Kentucky is for water to move downward to the water table, then through the saturated zone toward lower altitude, before discharging through springs/seeps into surface-water bodies (Price et al., 1962). For Na-SO 4 waters, Na may result from water that either infiltrated the ridge interior or discharged below the drainage basin (location MW-2 
and $\mathrm{MW}-11$ ) and $\mathrm{SO}_{4}{ }^{2-}$ from pyrite oxidation. Coal seams and rocks common to the area often contain pyrite (Wunsch, 1993).
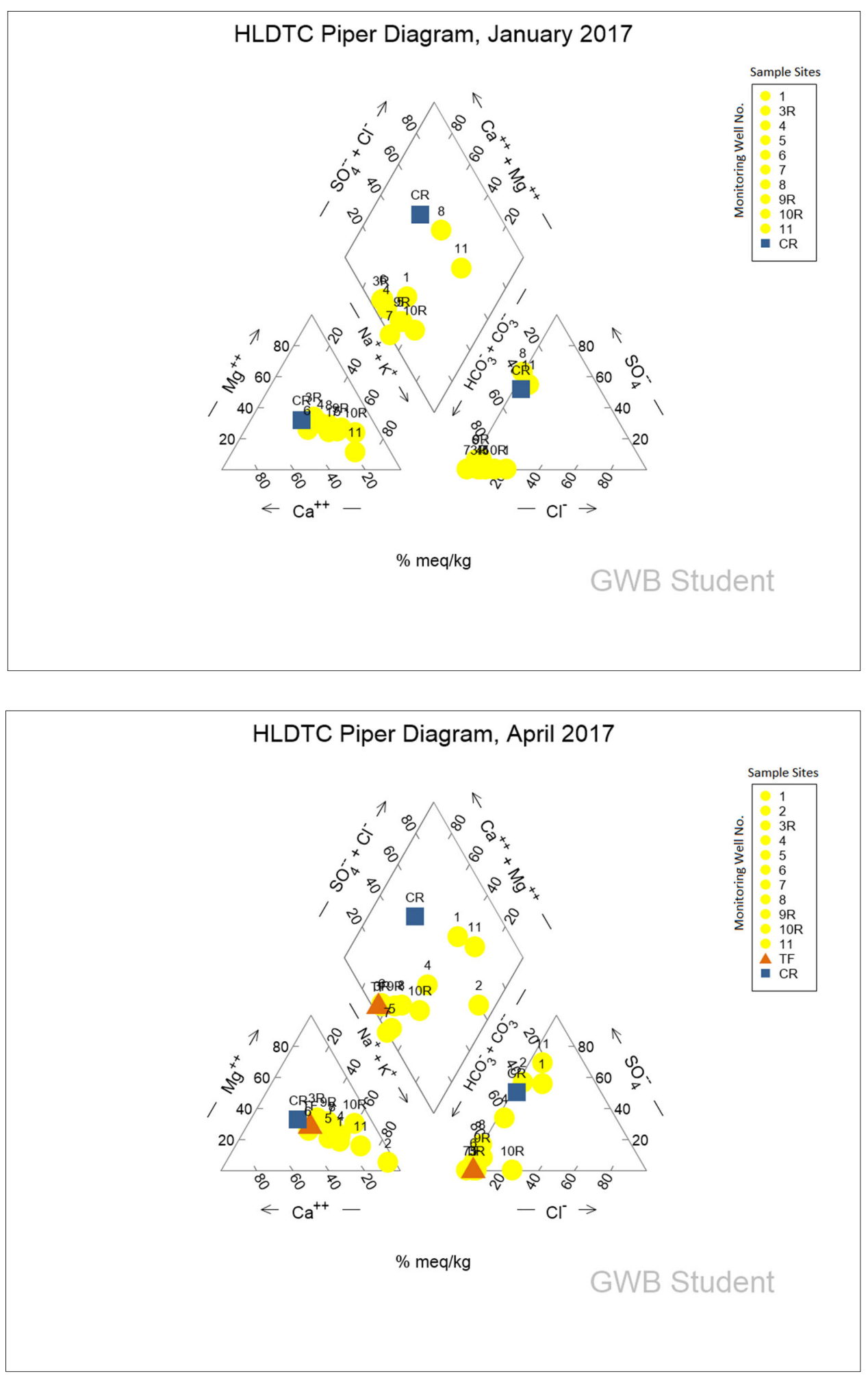

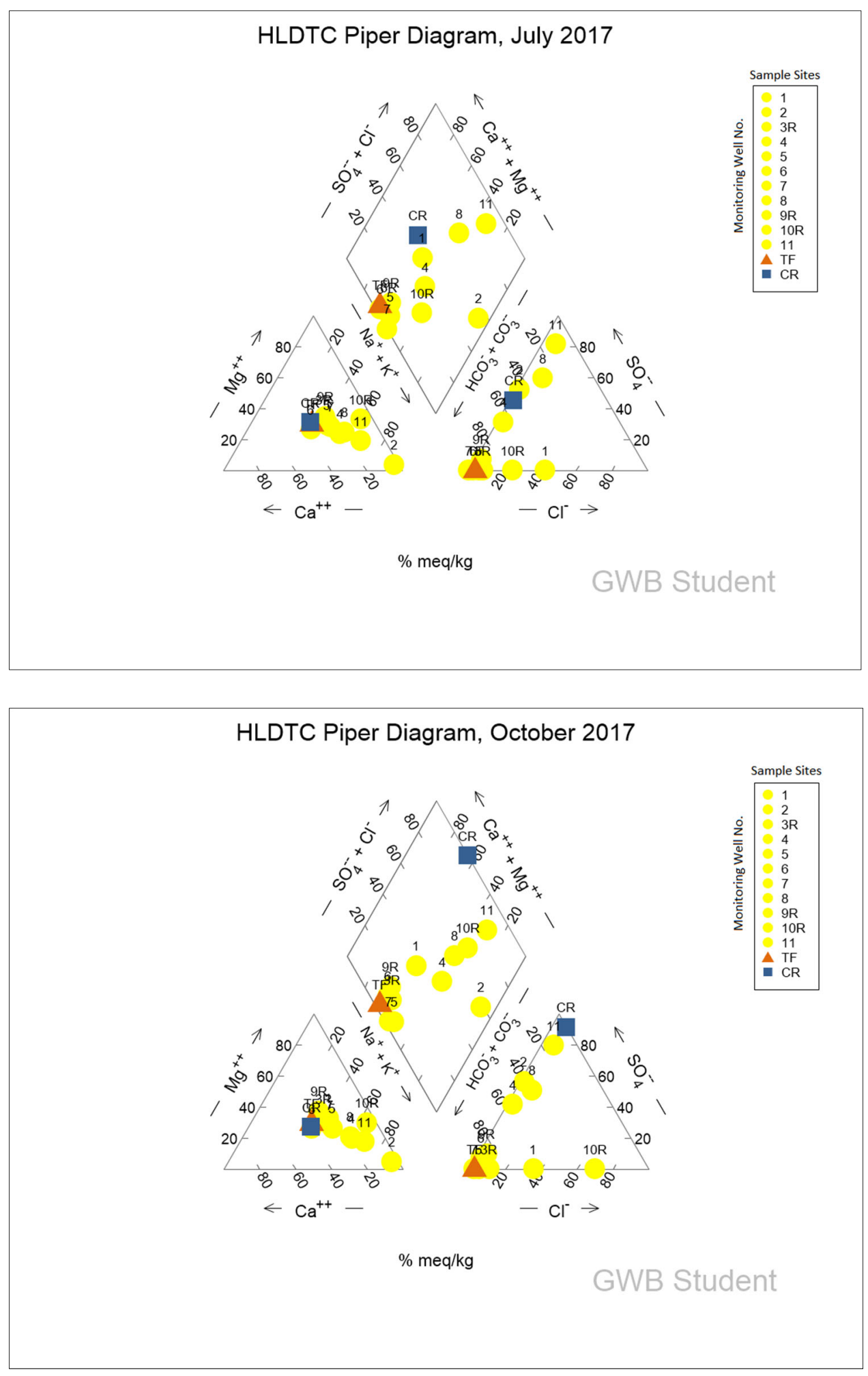


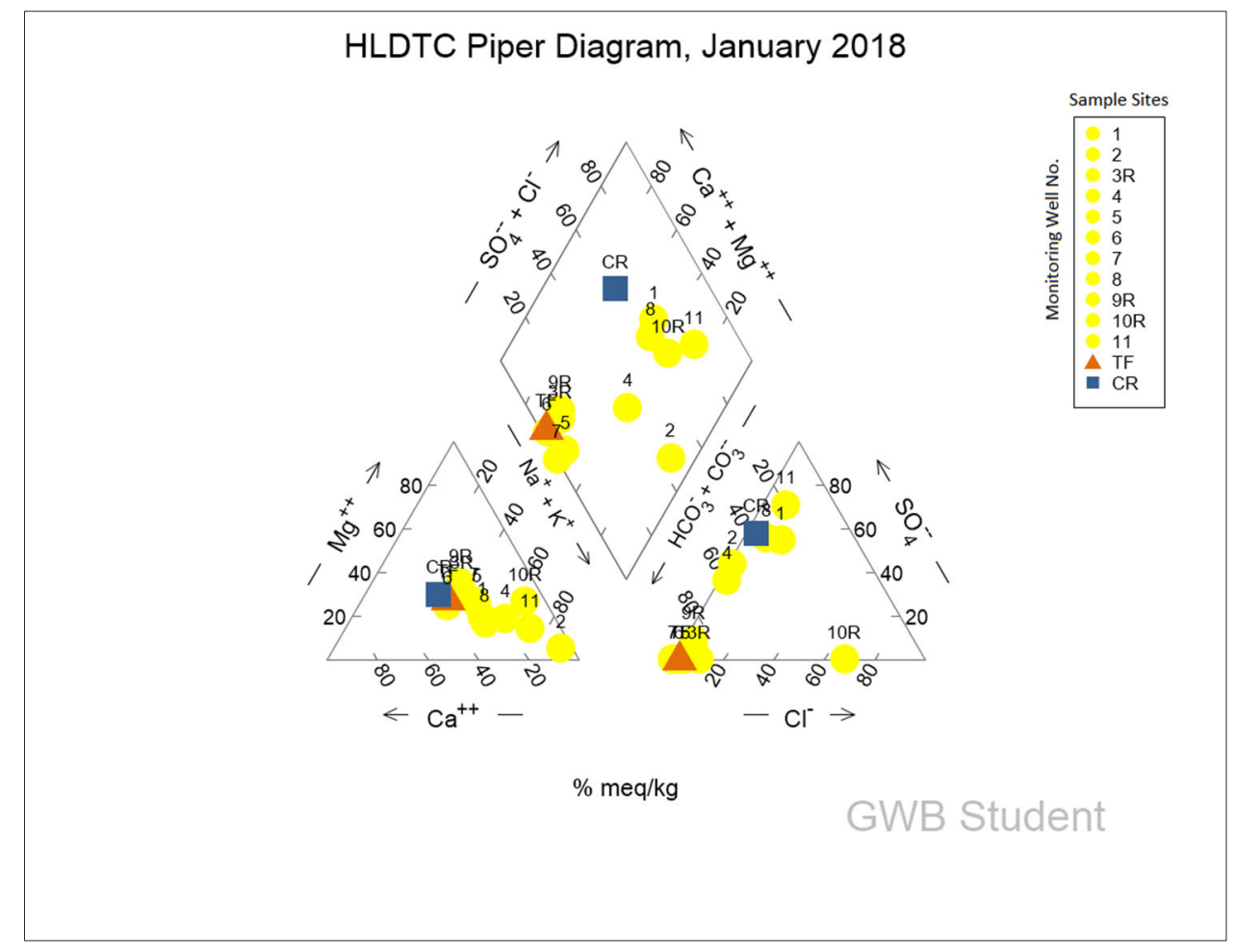

Figure 13: Quarterly Piper diagrams for HLDTC from January 2017 to January 2018.

Charge-balance errors (CBE\%) calculated by PHREEQC are presented in Table 8. The $\mathrm{CBE} \%$ site-wide mean value was $1.61 \%$ with a minimum of $-56 \%$ for MW-8 on April 8,2017 , and a maximum of $32.7 \%$ for MW-5 on January 6,2017 . The mean values for individual monitoring wells ranged from $-23.69 \%(\mathrm{MW}-1)$ to $17.26 \%(\mathrm{MW}-7)$, with four wells having $\mathrm{CBE} \%$ beyond $\pm 10 \%$ (MW-1 -23.69\%, MW-5 16.83\%, MW-7 17.26\%, MW$8-14.13 \%$ ). The tree farm well was $2.64 \%$ and Cumberland River $8.02 \%$.

The $\mathrm{CBE} \%$ calculation is performed as a check on conducted water chemistry analyses. In neutral waters, if all ions and cations present are analyzed, the error percentage would ideally be zero. If it is not, then there was either an error with chemical analysis and/or one or more ionic species present in a significant amount within the water was not identified. In a study of published journal articles, Fritz (1994) identified that the average $\mathrm{CBE} \%$ was positive with a mean $3.99 \%$. The positive value reflects that more cations are 
identified in water chemistry analysis than ions. Fritz proposed that the positive CBE\% was due to variances in the determination of alkalinity $\left(\mathrm{HCO}_{3}^{-}\right)$values.

A positive $\mathrm{CBE} \%$ trend is observed at HLDTC: nine of the 12 sites had positive $\mathrm{CBE} \%$ and three were negative (MW-1, MW-8, MW-9R). The monitoring wells with the highest positive CBE\% (MW-5 16.83\%, MW-6 9.85\%, MW-7 17.26\%) were completed at greater depths (11.9-13.6 m bls) into siltstones/shales, generally had higher $\mathrm{pH}(6.23-6.76)$ and alkalinity $(63.5-149.7 \mathrm{mg} / \mathrm{L})$ values, and were highest in DOC. In contrast, the three negative CBE\% monitoring wells (MW-1 -23.69\%, MW-8 -14.13\%, MW-9R -9.38\%) were shallowly completed (4.3-6.9 m bls) into friable sandstone, generally had lower $\mathrm{pH}$ (4.12-6.64) and alkalinity (3.3-106.6 $\mathrm{mg} / \mathrm{L}$ ) values, and DOC was below method detection limits. In both situations, the positive and negative $\mathrm{CBE} \%$ errors were likely from cations and anions that were not identified, although suspect inflection points (Table 1) indicate possible error in alkalinity titrations also (Fritz, 1994). Elevated positive CBE\% values may have reflected contributions from unanalyzed organic acid anions (Moumouni and Fryar, 2017), whereas elevated negative CBE\% values were associated with relatively dilute waters. 
Table 8. HLDTC Charge-Balance Error (CBE\%)

\begin{tabular}{|c|c|c|c|c|c|c|}
\hline Sample ID & & & MW-1 & & & Mean \\
\hline Quarter Date & $1 / 7 / 2017$ & 4/7/2017 & 7/8/2017 & $10 / 26 / 2017$ & $1 / 2 / 2018$ & \\
\hline CBE (\%) & -1.28 & -21.72 & -30.63 & -36.81 & -28.01 & -23.69 \\
\hline Sample ID & & & MW-2 & & & \\
\hline Quarter Date & $1 / 1 / 2017$ & 4/8/2017 & 7/8/2017 & $10 / 26 / 2017$ & $1 / 2 / 2018$ & \\
\hline CBE (\%) & -- & -10.57 & 9.8 & 2.81 & 10.4 & 3.11 \\
\hline Sample ID & & & MW-3R & & & \\
\hline Quarter Date & $1 / 6 / 2017$ & 4/7/2017 & 7/8/2017 & $10 / 26 / 2017$ & $1 / 2 / 2018$ & \\
\hline CBE (\%) & 4.68 & -9.07 & 11.38 & 26.43 & 5.85 & 7.85 \\
\hline Sample ID & & & MW-4 & & & \\
\hline Quarter Date & $1 / 6 / 2017$ & $4 / 7 / 2017$ & 7/9/2017 & $10 / 28 / 2017$ & $1 / 5 / 2018$ & \\
\hline CBE (\%) & 7.38 & -5.7 & 3.15 & 2.58 & 3.83 & 2.25 \\
\hline Sample ID & & & MW-5 & & & \\
\hline Quarter Date & $1 / 6 / 2017$ & 4/9/2017 & $7 / 10 / 2017$ & $10 / 27 / 2017$ & $1 / 4 / 2018$ & \\
\hline CBE (\%) & 32.7 & 1.17 & 26.39 & 15.72 & 8.17 & 16.83 \\
\hline Sample ID & & & MW-6 & & & \\
\hline Quarter Date & $1 / 5 / 2017$ & 4/9/2017 & $7 / 10 / 2017$ & $10 / 27 / 2017$ & $1 / 5 / 2018$ & \\
\hline CBE (\%) & 3.63 & -6.14 & 21.77 & 16.92 & 13.07 & 9.85 \\
\hline Sample ID & & & MW-7 & & & \\
\hline Quarter Date & $1 / 5 / 2017$ & 4/9/2017 & $7 / 10 / 2017$ & $10 / 27 / 2017$ & $1 / 5 / 2018$ & \\
\hline CBE (\%) & -2.42 & 19.94 & 22.4 & 28.26 & 18.14 & 17.26 \\
\hline Sample ID & & & MW-8 & & & \\
\hline Quarter Date & $1 / 7 / 2017$ & 4/8/2017 & 7/9/2017 & $10 / 27 / 2017$ & $1 / 3 / 2018$ & \\
\hline CBE (\%) & -7.61 & -56 & 0.35 & -0.87 & -6.51 & -14.13 \\
\hline Sample ID & & & MW-9R & & & \\
\hline Quarter Date & $1 / 7 / 2017$ & 4/9/2017 & 7/8/2017 & $10 / 26 / 2017$ & $1 / 4 / 2018$ & \\
\hline CBE (\%) & -21.11 & -9.42 & -11.87 & 2.12 & -6.63 & -9.38 \\
\hline Sample ID & & & MW-10R & & & \\
\hline Quarter Date & $1 / 8 / 2017$ & 4/8/2017 & 7/9/2017 & $10 / 27 / 2017$ & $1 / 1 / 2018$ & \\
\hline CBE (\%) & -7.4 & -4.23 & -4.97 & 22.76 & -4.82 & 0.27 \\
\hline Sample ID & & & MW-11 & & & \\
\hline Quarter Date & $1 / 8 / 2017$ & 4/10/2017 & 7/9/2017 & $10 / 28 / 2017$ & $1 / 3 / 2018$ & \\
\hline CBE (\%) & 0.06 & -6.8 & 2.96 & -5.67 & 11.94 & 0.50 \\
\hline Sample ID & & & TF & & & \\
\hline Quarter Date & $1 / 1 / 2017$ & $4 / 10 / 2017$ & $7 / 10 / 2017$ & $10 / 28 / 2017$ & $1 / 4 / 2018$ & \\
\hline CBE (\%) & -- & -0.55 & 13.76 & -0.06 & -2.59 & 2.64 \\
\hline Sample ID & & & CR & & & \\
\hline Quarter Dat & $1 / 8 / 2017$ & 4/10/2017 & $7 / 12 / 2017$ & $10 / 25 / 2017$ & $1 / 1 / 2018$ & \\
\hline CBE (\%) & -3.45 & -6.18 & 5.17 & 33.05 & 11.53 & 8.02 \\
\hline \multicolumn{7}{|l|}{ Notes: } \\
\hline & & & & & & \\
\hline & $\begin{array}{r}\text { Italicized } \\
\text { blue }\end{array}$ & $\begin{array}{l}\text { Suspect } i \\
|\mathrm{CBE}|>\end{array}$ & UII & . & & \\
\hline
\end{tabular}


Saturation index (SI) values calculated by PHREEQC are presented in Table 9. Maximum values are indicated by red text, minimum by blue, undersaturated (negative) values by non-bold text and supersaturated (positive) by bold text. The following phases were undersaturated for all samples: calcite, strontianite, and witherite (carbonates); anhydrite, celestite, gypsum, and melanterite (sulfates); manganite, pyrochroite, and pyrolusite (Mn (oxyhydr)oxides); amorphous $\mathrm{SiO}_{2}$; and halite. The minerals that were supersaturated for at least one sample were dolomite and siderite (carbonates); goethite, hematite, and amorphous $\mathrm{Fe}(\mathrm{OH})_{3} \quad(\mathrm{Fe} \quad$ (oxyhydr)oxides); hausmannite (Mn (oxyhydr)oxide); barite; quartz; and fluorite. Barite and fluorite were each supersaturated for one sample: barite in MW-2 (April 2017) and fluorite in MW-7 (July 2017). Again, data from MW-2 are suspect due to the potential for improper well development. Siderite was supersaturated in MW-5 and MW-7 for all sampling events, whereas dolomite was only supersaturated in the Cumberland River samples (January 2017, July 2017, and January 2018). Hausmannite was supersaturated in MW-7 for all sampling events. At least one of the Fe (oxyhydr)oxide phases was at or near supersaturation for each sampling site and event, with the order of SI values increasing from least- to most-crystalline phases (amorphous $\mathrm{Fe}(\mathrm{OH})_{3}<$ goethite $<$ hematite). Quartz was at or near supersaturation for all samples, including the tree farm and the Cumberland River. There were no discernible spatial or seasonal trends. 
Table 9. HLDTC Mineral Saturation Indexes (SI)

\begin{tabular}{|c|c|c|c|c|c|c|}
\hline \multirow{4}{*}{$\begin{array}{l}\text { Mineral } \\
\text { Phase }\end{array}$} & \multirow{4}{*}{$\begin{array}{l}\text { Chemical } \\
\text { Formula } \\
\text { Sample ID } \\
\text { Quarter Date }\end{array}$} & \multirow{3}{*}{\multicolumn{5}{|c|}{$\begin{array}{c}\text { Saturation Index (SI) } \\
\text { MW-1 }\end{array}$}} \\
\hline & & & & & & \\
\hline & & & & & & \\
\hline & & $1 / 7 / 2017$ & $4 / 7 / 2017$ & $7 / 8 / 2017$ & $10 / 26 / 2017$ & $1 / 2 / 2018$ \\
\hline Anhydrite & $\mathrm{CaSO}_{4}$ & -- & -4.61 & -- & -- & -4.65 \\
\hline Barite & $\mathrm{BaSO}_{4}$ & -- & -1.09 & -- & -- & -1.14 \\
\hline Calcite & $\mathrm{CaCO}_{3}$ & -5.47 & -6.21 & -6.01 & -6.17 & -6.19 \\
\hline Celestite & $\mathrm{SrSO}_{4}$ & -- & -- & -- & -- & -- \\
\hline $\mathrm{CO} 2(\mathrm{~g})$ & $\mathrm{CO}_{2}$ & -1.25 & -0.44 & -1.25 & -0.81 & -0.06 \\
\hline Dolomite & $\mathrm{CaMg}(\mathrm{CO} 3)_{2}$ & -11.05 & -12.58 & -11.93 & -12.19 & -12.59 \\
\hline $\mathrm{Fe}(\mathrm{OH}) 3(\mathrm{a})$ & $\mathrm{Fe}(\mathrm{OH})_{3}$ & -- & -7.8 & -6.55 & -7.09 & -- \\
\hline Fluorite & $\mathrm{CaF}_{2}$ & -- & -- & -- & -- & -- \\
\hline Goethite & $\mathrm{FeOOH}$ & -- & -2.35 & -0.94 & -1.45 & 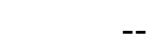 \\
\hline Gypsum & $\mathrm{CaSO}_{4}: 2 \mathrm{H}_{2} \mathrm{O}$ & -- & -4.17 & -- & -- & -4.21 \\
\hline Halite & $\mathrm{NaCl}$ & -10.29 & -9.94 & -10.32 & -10.44 & -10.08 \\
\hline Hausmannite & $\mathrm{Mn}_{3} \mathrm{O}_{4}$ & -32.73 & -39.71 & -34.15 & -36.58 & -42.62 \\
\hline Hematite & $\mathrm{Fe}_{2} \mathrm{O}_{3}$ & -- & -2.74 & 0.1 & -0.93 & -- \\
\hline Manganite & $\mathrm{MnOOH}$ & -11.86 & -14.45 & -12.79 & -13.75 & -15.47 \\
\hline Melanterite & $\mathrm{FeSO}_{4}: 7 \mathrm{H}_{2} \mathrm{O}$ & -- & -8.3 & -- & -- & -- \\
\hline Pyrochroite & $\mathrm{Mn}(\mathrm{OH})_{2}$ & -10.79 & -12.61 & -11.5 & -12.2 & -13.45 \\
\hline Pyrolusite & $\mathrm{MnO}_{2}: \mathrm{H}_{2} \mathrm{O}$ & -20.81 & -24.16 & -21.22 & -22.32 & -25.38 \\
\hline Quartz & $\mathrm{SiO}_{2}$ & 0.07 & -0.03 & -0.01 & -0.05 & -0.04 \\
\hline Siderite & $\mathrm{FeCO}_{3}$ & -- & -5.72 & -5.89 & -5.74 & -- \\
\hline $\mathrm{SiO} 2(\mathrm{a})$ & $\mathrm{SiO}_{2}$ & -1.28 & -1.38 & -1.33 & -1.36 & -1.39 \\
\hline Strontianite & $\mathrm{SrCO}_{3}$ & -7 & -- & -- & -- & -- \\
\hline \multirow[t]{3}{*}{ Witherite } & $\mathrm{BaCO}_{3}$ & -7.57 & -8.32 & -8.01 & -8.1 & -8.3 \\
\hline & Sample ID & & & MW-2 & & \\
\hline & Quarter Date & $1 / 1 / 2017$ & $4 / 8 / 2017$ & $7 / 8 / 2017$ & $10 / 26 / 2017$ & $1 / 2 / 2018$ \\
\hline Anhydrite & $\mathrm{CaSO}_{4}$ & -- & -2.91 & -3.38 & -3.51 & -3.76 \\
\hline Barite & $\mathrm{BaSO}_{4}$ & -- & 0.19 & -0.3 & -0.36 & -0.79 \\
\hline Calcite & $\mathrm{CaCO}_{3}$ & -- & -2.31 & -2.77 & -3.29 & -3.54 \\
\hline Celestite & $\mathrm{SrSO}_{4}$ & -- & -2.86 & -3.38 & -3.44 & -3.65 \\
\hline $\mathrm{CO} 2(\mathrm{~g})$ & $\mathrm{CO}_{2}$ & -- & -1.35 & -1.5 & -1.39 & -1.42 \\
\hline Dolomite & $\mathrm{CaMg}(\mathrm{CO} 3)_{2}$ & -- & -4.56 & -5.43 & -6.52 & -7.15 \\
\hline $\mathrm{Fe}(\mathrm{OH}) 3(\mathrm{a})$ & $\mathrm{Fe}(\mathrm{OH})_{3}$ & -- & -0.31 & -1.8 & -1.39 & -1.83 \\
\hline Fluorite & $\mathrm{CaF}_{2}$ & -- & -- & -- & -3.48 & -- \\
\hline Goethite & $\mathrm{FeOOH}$ & -- & 5.22 & 3.76 & 4.14 & 3.55 \\
\hline Gypsum & $\mathrm{CaSO}_{4}: 2 \mathrm{H}_{2} \mathrm{O}$ & -- & -2.49 & -2.97 & -3.1 & -3.3 \\
\hline Halite & $\mathrm{NaCl}$ & -- & -7.64 & -8.14 & -8.49 & -8.76 \\
\hline Hausmannite & $\mathrm{Mn}_{3} \mathrm{O}_{4}$ & -- & -19.37 & -20.79 & -23.89 & -26.31 \\
\hline Hematite & $\mathrm{Fe}_{2} \mathrm{O}_{3}$ & -- & 12.39 & 9.48 & 10.24 & 9.04 \\
\hline Manganite & $\mathrm{MnOOH}$ & -- & -7.11 & -7.67 & -8.74 & -9.24 \\
\hline Melanterite & $\mathrm{FeSO}_{4}: 7 \mathrm{H}_{2} \mathrm{O}$ & -- & -6.19 & -7.8 & -6.59 & -6.8 \\
\hline Pyrochroite & $\mathrm{Mn}(\mathrm{OH})_{2}$ & -- & -7.46 & -7.96 & -8.74 & -9.14 \\
\hline Pyrolusite & $\mathrm{MnO}_{2}: \mathrm{H}_{2} \mathrm{O}$ & -- & -14.31 & -14.8 & -16.27 & -17.53 \\
\hline Quartz & $\mathrm{SiO}_{2}$ & -- & 0.25 & 0.23 & 0.28 & 0.36 \\
\hline Siderite & $\mathrm{FeCO}_{3}$ & -- & -1.36 & -2.94 & -2.13 & -2.45 \\
\hline $\mathrm{SiO} 2(\mathrm{a})$ & $\mathrm{SiO}_{2}$ & -- & -1.09 & -1.1 & -1.06 & -1 \\
\hline Strontianite & $\mathrm{SrCO}_{3}$ & -- & -3.85 & -4.36 & -4.81 & -5.01 \\
\hline Witherite & $\mathrm{BaCO}_{3}$ & -- & -4.82 & -5.29 & -5.73 & -6.21 \\
\hline
\end{tabular}


Table 9. HLDTC Mineral Saturation Indexes (SI) (cont.)

\begin{tabular}{|c|c|c|c|c|c|c|}
\hline & Sample ID & \multicolumn{5}{|c|}{ MW-3R } \\
\hline & Quarter Date & $1 / 6 / 2017$ & $4 / 7 / 2017$ & $7 / 8 / 2017$ & $10 / 26 / 2017$ & $1 / 2 / 2018$ \\
\hline Anhydrite & $\mathrm{CaSO}_{4}$ & -- & -- & -- & -- & -- \\
\hline Barite & $\mathrm{BaSO}_{4}$ & -- & -- & -- & -- & -- \\
\hline Calcite & $\mathrm{CaCO}_{3}$ & -2.85 & -2.97 & -3.13 & -3.49 & -3.37 \\
\hline Celestite & $\mathrm{SrSO}_{4}$ & -- & -0.08 & -0.05 & -0.06 & -0.01 \\
\hline $\mathrm{CO} 2(\mathrm{~g})$ & $\mathrm{CO}_{2}$ & -1.75 & -1.55 & -1.63 & -1.55 & -1.32 \\
\hline Dolomite & $\mathrm{CaMg}(\mathrm{CO} 3)_{2}$ & -5.8 & -5.91 & -6.17 & -6.94 & -6.79 \\
\hline $\mathrm{Fe}(\mathrm{OH}) 3(\mathrm{a})$ & $\mathrm{Fe}(\mathrm{OH})_{3}$ & 0.91 & 0.69 & 0.57 & -0.06 & -0.27 \\
\hline Fluorite & $\mathrm{CaF}_{2}$ & -- & -- & -- & -- & -- \\
\hline Goethite & $\mathrm{FeOOH}$ & 6.3 & 6.18 & 6.13 & 5.48 & 5.15 \\
\hline Gypsum & $\mathrm{CaSO}_{4}: 2 \mathrm{H}_{2} \mathrm{O}$ & -- & -- & -- & -- & -- \\
\hline Halite & $\mathrm{NaCl}$ & -9.61 & -9.62 & -9.5 & -9.51 & -9.57 \\
\hline Hausmannite & $\mathrm{Mn}_{3} \mathrm{O}_{4}$ & -20.69 & -21.16 & -21.14 & -23.04 & -24.28 \\
\hline Hematite & $\mathrm{Fe}_{2} \mathrm{O}_{3}$ & 14.55 & 14.31 & 14.22 & 12.91 & 12.24 \\
\hline Manganite & $\mathrm{MnOOH}$ & -7.28 & -7.71 & -7.88 & -8.53 & -8.69 \\
\hline Melanterite & $\mathrm{FeSO}_{4}: 7 \mathrm{H}_{2} \mathrm{O}$ & -- & -- & -- & -- & -- \\
\hline Pyrochroite & $\mathrm{Mn}(\mathrm{OH})_{2}$ & -7.51 & -7.83 & -7.94 & -8.37 & -8.48 \\
\hline Pyrolusite & $\mathrm{MnO}_{2}: \mathrm{H}_{2} \mathrm{O}$ & -15.19 & -15.28 & -15.2 & -16.18 & -16.92 \\
\hline Quartz & $\mathrm{SiO}_{2}$ & 0.47 & 0.39 & 0.41 & 0.4 & 0.46 \\
\hline Siderite & $\mathrm{FeCO}_{3}$ & -0.37 & -0.31 & -0.48 & -0.81 & -0.69 \\
\hline $\mathrm{SiO} 2(\mathrm{a})$ & $\mathrm{SiO}_{2}$ & -0.89 & -0.95 & -0.92 & -0.94 & -0.89 \\
\hline Strontianite & $\mathrm{SrCO}_{3}$ & -4.17 & -4.35 & -4.54 & -4.82 & -4.69 \\
\hline \multirow[t]{3}{*}{ Witherite } & $\mathrm{BaCO}_{3}$ & -4.96 & -5.05 & -5.32 & -5.61 & -5.48 \\
\hline & Sample ID & & & MW-4 & & \\
\hline & Quarter Date & $1 / 6 / 2017$ & $4 / 7 / 2017$ & $7 / 9 / 2017$ & $10 / 28 / 2017$ & $1 / 5 / 2018$ \\
\hline Anhydrite & $\mathrm{CaSO}_{4}$ & - & -3.13 & -3.14 & -3.15 & -3.28 \\
\hline Barite & $\mathrm{BaSO}_{4}$ & -- & -0.25 & -0.12 & -0.24 & -0.4 \\
\hline Calcite & $\mathrm{CaCO}_{3}$ & -2.05 & -2.18 & -2.11 & -2.55 & -2.63 \\
\hline Celestite & $\mathrm{SrSO}_{4}$ & -- & -3.2 & -3.27 & -3.24 & -3.34 \\
\hline $\mathrm{CO} 2(\mathrm{~g})$ & $\mathrm{CO}_{2}$ & -1.58 & -1.54 & -1.58 & -1.46 & -1.55 \\
\hline Dolomite & $\mathrm{CaMg}(\mathrm{CO} 3)_{2}$ & -4.16 & -4.38 & -4.18 & -5.08 & -5.36 \\
\hline $\mathrm{Fe}(\mathrm{OH}) 3(\mathrm{a})$ & $\mathrm{Fe}(\mathrm{OH})_{3}$ & $\mathbf{0 . 5 3}$ & 0.28 & 0.54 & 0.05 & -0.06 \\
\hline Fluorite & $\mathrm{CaF}_{2}$ & -- & -- & -- & -3.32 & -3.61 \\
\hline Goethite & $\mathrm{FeOOH}$ & 5.97 & 5.77 & 6.13 & 5.59 & 5.34 \\
\hline Gypsum & $\mathrm{CaSO}_{4}: 2 \mathrm{H}_{2} \mathrm{O}$ & - & -2.7 & -2.74 & -2.73 & -2.83 \\
\hline Halite & $\mathrm{NaCl}$ & -8.88 & -8.64 & -8.76 & -8.73 & -8.79 \\
\hline Hausmannite & $\mathrm{Mn}_{3} \mathrm{O}_{4}$ & -17.85 & -17.72 & -16.77 & -19.16 & -20.11 \\
\hline Hematite & $\mathrm{Fe}_{2} \mathrm{O}_{3}$ & 13.89 & 13.5 & 14.24 & 13.14 & 12.62 \\
\hline Manganite & $\mathrm{MnOOH}$ & -6.39 & -6.49 & -6.41 & -7.15 & -7.13 \\
\hline Melanterite & $\mathrm{FeSO}_{4}: 7 \mathrm{H}_{2} \mathrm{O}$ & -- & -6.06 & -6.05 & -5.7 & -5.84 \\
\hline Pyrochroite & $\mathrm{Mn}(\mathrm{OH})_{2}$ & -6.74 & -6.8 & -6.74 & -7.26 & -7.27 \\
\hline Pyrolusite & $\mathrm{MnO}_{2}: \mathrm{H}_{2} \mathrm{O}$ & -14 & -13.88 & -13.31 & -14.53 & -15.11 \\
\hline Quartz & $\mathrm{SiO}_{2}$ & 0.45 & 0.45 & 0.47 & 0.45 & 0.51 \\
\hline Siderite & $\mathrm{FeCO}_{3}$ & -0.71 & -0.91 & -0.74 & -0.86 & -1.04 \\
\hline $\mathrm{SiO} 2(\mathrm{a})$ & $\mathrm{SiO}_{2}$ & -0.9 & -0.89 & -0.85 & -0.88 & -0.85 \\
\hline Strontianite & $\mathrm{SrCO}_{3}$ & -3.71 & -3.85 & -3.83 & -4.23 & -4.27 \\
\hline Witherite & $\mathrm{BaCO}_{3}$ & -4.82 & -4.93 & -4.67 & -5.24 & -5.38 \\
\hline
\end{tabular}


Table 9. HLDTC Mineral Saturation Indexes (SI) (cont.)

\begin{tabular}{|c|c|c|c|c|c|c|}
\hline & Sample ID & \multicolumn{5}{|c|}{ MW-5 } \\
\hline & Quarter Date & $1 / 6 / 2017$ & $4 / 9 / 2017$ & $7 / 10 / 2017$ & $10 / 27 / 2017$ & $1 / 4 / 2018$ \\
\hline Anhydrite & $\mathrm{CaSO}_{4}$ & -- & -- & -- & -- & -- \\
\hline Barite & $\mathrm{BaSO}_{4}$ & -- & -- & -- & -- & -- \\
\hline Calcite & $\mathrm{CaCO}_{3}$ & -2.52 & -2.78 & -2.35 & -2.72 & -2.8 \\
\hline Celestite & $\mathrm{SrSO}_{4}$ & -- & -- & -- & -- & -- \\
\hline $\mathrm{CO} 2(\mathrm{~g})$ & $\mathrm{CO}_{2}$ & -1.8 & -1.43 & -1.83 & -1.45 & -1.4 \\
\hline Dolomite & $\mathrm{CaMg}(\mathrm{CO} 3)_{2}$ & -5.1 & -5.73 & -4.61 & -5.45 & -5.7 \\
\hline $\mathrm{Fe}(\mathrm{OH}) 3(\mathrm{a})$ & $\mathrm{Fe}(\mathrm{OH})_{3}$ & 1.75 & 1.19 & 2.17 & 1.1 & 0.92 \\
\hline Fluorite & $\mathrm{CaF}_{2}$ & -- & -- & -- & -3.76 & -3.74 \\
\hline Goethite & $\mathrm{FeOOH}$ & 7.1 & 6.7 & 7.85 & 6.63 & 6.31 \\
\hline Gypsum & $\mathrm{CaSO}_{4}: 2 \mathrm{H}_{2} \mathrm{O}$ & -- & -- & -- & -- & -- \\
\hline Halite & $\mathrm{NaCl}$ & -8.78 & -9.48 & -9.25 & -9.27 & -9.34 \\
\hline Hausmannite & $\mathrm{Mn}_{3} \mathrm{O}_{4}$ & -17.63 & -18.94 & -14.78 & -18.63 & -20.02 \\
\hline Hematite & $\mathrm{Fe}_{2} \mathrm{O}_{3}$ & 16.13 & 15.36 & 17.69 & 15.23 & 14.56 \\
\hline Manganite & $\mathrm{MnOOH}$ & -6.1 & -7.01 & -5.92 & -6.96 & -7.09 \\
\hline Melanterite & $\mathrm{FeSO}_{4}: 7 \mathrm{H}_{2} \mathrm{O}$ & -- & -- & -- & -- & -- \\
\hline Pyrochroite & $\mathrm{Mn}(\mathrm{OH})_{2}$ & -6.47 & -7.16 & -6.37 & -7.08 & -7.18 \\
\hline Pyrolusite & $\mathrm{MnO}_{2}: \mathrm{H}_{2} \mathrm{O}$ & -14.09 & -14.45 & -12.3 & -14.34 & -15.17 \\
\hline Quartz & $\mathrm{SiO}_{2}$ & 0.54 & 0.37 & 0.45 & 0.46 & 0.53 \\
\hline Siderite & $\mathrm{FeCO}_{3}$ & 0.29 & 0.26 & 0.49 & 0.18 & 0.14 \\
\hline $\mathrm{SiO} 2(\mathrm{a})$ & $\mathrm{SiO}_{2}$ & -0.83 & -0.97 & -0.85 & -0.88 & -0.83 \\
\hline Strontianite & $\mathrm{SrCO}_{3}$ & -- & -- & -- & -- & -- \\
\hline \multirow[t]{3}{*}{ Witherite } & $\mathrm{BaCO}_{3}$ & -4.65 & -4.82 & -4.62 & -4.9 & -4.97 \\
\hline & Sample ID & & & MW-6 & & \\
\hline & Quarter Date & $1 / 5 / 2017$ & $4 / 9 / 2017$ & $7 / 10 / 2017$ & $10 / 27 / 2017$ & $1 / 5 / 2018$ \\
\hline Anhydrite & $\mathrm{CaSO}_{4}$ & -3.72 & -3.87 & -- & -3.85 & -- \\
\hline Barite & $\mathrm{BaSO}_{4}$ & -0.35 & -0.41 & -- & -0.38 & -- \\
\hline Calcite & $\mathrm{CaCO}_{3}$ & -1.67 & -1.73 & -1.97 & -2.13 & -2.08 \\
\hline Celestite & $\mathrm{SrSO}_{4}$ & -3.14 & -3.37 & -- & -3.36 & -- \\
\hline $\mathrm{CO} 2(\mathrm{~g})$ & $\mathrm{CO}_{2}$ & -1.6 & -1.51 & -1.66 & -1.46 & -1.39 \\
\hline Dolomite & $\mathrm{CaMg}(\mathrm{CO} 3)_{2}$ & -3.57 & -3.65 & -4.1 & -4.43 & -4.43 \\
\hline $\mathrm{Fe}(\mathrm{OH}) 3(\mathrm{a})$ & $\mathrm{Fe}(\mathrm{OH})_{3}$ & 2.29 & 2.2 & 2.15 & 1.63 & 1.66 \\
\hline Fluorite & $\mathrm{CaF}_{2}$ & -- & -- & -- & -- & -- \\
\hline Goethite & $\mathrm{FeOOH}$ & 7.73 & 7.73 & -- & 7.16 & -- \\
\hline Gypsum & $\mathrm{CaSO}_{4}: 2 \mathrm{H}_{2} \mathrm{O}$ & -3.28 & -3.46 & -- & -3.43 & -- \\
\hline Halite & $\mathrm{NaCl}$ & -9.28 & -9.41 & -9.35 & -9.25 & -9.4 \\
\hline Hausmannite & $\mathrm{Mn}_{3} \mathrm{O}_{4}$ & -18.9 & -18.69 & -18.94 & -20.52 & -21.07 \\
\hline Hematite & $\mathrm{Fe}_{2} \mathrm{O}_{3}$ & 17.41 & 17.43 & 17.34 & 16.27 & 16.12 \\
\hline Manganite & $\mathrm{MnOOH}$ & -6.71 & -6.86 & -6.97 & -7.52 & -7.49 \\
\hline Melanterite & $\mathrm{FeSO}_{4}: 7 \mathrm{H}_{2} \mathrm{O}$ & -5.38 & -5.52 & -- & -5.37 & -- \\
\hline Pyrochroite & $\mathrm{Mn}(\mathrm{OH})_{2}$ & -7.23 & -7.34 & -7.4 & -7.77 & -7.75 \\
\hline Pyrolusite & $\mathrm{MnO}_{2}: \mathrm{H}_{2} \mathrm{O}$ & -14.1 & -13.89 & -14.02 & -14.83 & -15.2 \\
\hline Quartz & $\mathrm{SiO}_{2}$ & 0.31 & 0.24 & 0.29 & 0.27 & 0.34 \\
\hline Siderite & $\mathrm{FeCO}_{3}$ & 0.85 & 0.86 & 0.71 & 0.58 & 0.69 \\
\hline $\mathrm{SiO} 2(\mathrm{a})$ & $\mathrm{SiO}_{2}$ & -1.04 & -1.09 & -1.04 & -1.06 & -1.01 \\
\hline Strontianite & $\mathrm{SrCO}_{3}$ & -2.68 & -2.81 & -- & -3.23 & -3.19 \\
\hline Witherite & $\mathrm{BaCO}_{3}$ & -3.93 & -3.87 & -4.12 & -4.27 & -4.18 \\
\hline
\end{tabular}


Table 9. HLDTC Mineral Saturation Indexes (SI) (cont.)

\begin{tabular}{|c|c|c|c|c|c|c|}
\hline & Sample ID & \multicolumn{5}{|c|}{ MW-7 } \\
\hline & Quarter Date & $1 / 5 / 2017$ & $4 / 9 / 2017$ & $7 / 10 / 2017$ & $10 / 27 / 2017$ & $1 / 5 / 2018$ \\
\hline Anhydrite & $\mathrm{CaSO}_{4}$ & -- & -- & -- & -- & -- \\
\hline Barite & $\mathrm{BaSO}_{4}$ & -- & -- & -- & -- & -- \\
\hline Calcite & $\mathrm{CaCO}_{3}$ & -2.27 & -2.43 & -2.51 & -2.73 & -2.61 \\
\hline Celestite & $\mathrm{SrSO}_{4}$ & -- & -- & -- & -- & -- \\
\hline $\mathrm{CO} 2(\mathrm{~g})$ & $\mathrm{CO}_{2}$ & -1.74 & -1.95 & -1.89 & -1.72 & -1.64 \\
\hline Dolomite & $\mathrm{CaMg}(\mathrm{CO} 3)_{2}$ & -4.56 & -4.82 & -4.97 & -5.45 & -5.3 \\
\hline $\mathrm{Fe}(\mathrm{OH}) 3(\mathrm{a})$ & $\mathrm{Fe}(\mathrm{OH})_{3}$ & 2.59 & 2.79 & 2.51 & 1.92 & 1.95 \\
\hline Fluorite & $\mathrm{CaF}_{2}$ & -- & -4.06 & 8.09 & -- & -3.86 \\
\hline Goethite & $\mathrm{FeOOH}$ & 8.05 & 8.35 & -- & 7.41 & 7.38 \\
\hline Gypsum & $\mathrm{CaSO}_{4}: 2 \mathrm{H}_{2} \mathrm{O}$ & -- & -- & -- & -- & -- \\
\hline Halite & $\mathrm{NaCl}$ & -16.26 & -15.1 & -15.59 & -17.69 & -18.08 \\
\hline Hausmannite & $\mathrm{Mn}_{3} \mathrm{O}_{4}$ & 18.04 & 18.66 & 18.15 & 16.79 & 16.71 \\
\hline Hematite & $\mathrm{Fe}_{2} \mathrm{O}_{3}$ & -8.72 & -8.27 & -8.58 & -9.85 & -9.77 \\
\hline Manganite & $\mathrm{MnOOH}$ & -5.83 & -5.66 & -5.91 & -6.48 & -6.46 \\
\hline Melanterite & $\mathrm{FeSO}_{4}: 7 \mathrm{H}_{2} \mathrm{O}$ & -- & -- & -- & -- & -- \\
\hline Pyrochroite & $\mathrm{Mn}(\mathrm{OH})_{2}$ & -6.42 & -6.28 & -6.44 & -6.81 & -6.81 \\
\hline Pyrolusite & $\mathrm{MnO}_{2}: \mathrm{H}_{2} \mathrm{O}$ & -13.1 & -12.46 & -12.7 & -13.84 & -14.08 \\
\hline Quartz & $\mathrm{SiO}_{2}$ & 0.19 & 0.11 & 0.13 & 0.14 & 0.19 \\
\hline Siderite & $\mathrm{FeCO}_{3}$ & 0.94 & 0.87 & 0.73 & 0.54 & 0.65 \\
\hline $\mathrm{SiO} 2(\mathrm{a})$ & $\mathrm{SiO}_{2}$ & -1.16 & -1.22 & -1.2 & -1.2 & -1.17 \\
\hline Strontianite & $\mathrm{SrCO}_{3}$ & -3.54 & -3.77 & -3.89 & -4.13 & -3.92 \\
\hline \multirow[t]{3}{*}{ Witherite } & $\mathrm{BaCO}_{3}$ & -4.15 & -4.3 & -4.47 & -4.65 & -4.53 \\
\hline & Sample ID & & & MW-8 & & \\
\hline & Quarter Date & $1 / 7 / 2017$ & $4 / 8 / 2017$ & $7 / 9 / 2017$ & $10 / 27 / 2017$ & $1 / 3 / 2018$ \\
\hline Anhydrite & $\mathrm{CaSO}_{4}$ & -4.78 & -4.79 & -4.7 & -4.64 & -4.35 \\
\hline Barite & $\mathrm{BaSO}_{4}$ & -1.11 & -0.96 & -0.95 & -1.12 & -1.11 \\
\hline Calcite & $\mathrm{CaCO}_{3}$ & -5.84 & -4.97 & -5.59 & -5.44 & -5.68 \\
\hline Celestite & $\mathrm{SrSO}_{4}$ & -- & -- & -- & -- & -- \\
\hline $\mathrm{CO} 2(\mathrm{~g})$ & $\mathrm{CO}_{2}$ & -1.28 & -0.4 & -1.55 & -1.23 & -0.88 \\
\hline Dolomite & $\mathrm{CaMg}(\mathrm{CO} 3)_{2}$ & -11.7 & -9.93 & -11.07 & -10.81 & -11.64 \\
\hline $\mathrm{Fe}(\mathrm{OH}) 3(\mathrm{a})$ & $\mathrm{Fe}(\mathrm{OH})_{3}$ & -4.91 & -4.36 & -3.21 & -3.28 & -5.21 \\
\hline Fluorite & $\mathrm{CaF}_{2}$ & -- & -- & -- & -- & -- \\
\hline Goethite & $\mathrm{FeOOH}$ & 0.47 & 1.02 & 2.39 & 2.34 & 0.23 \\
\hline Gypsum & $\mathrm{CaSO}_{4}: 2 \mathrm{H}_{2} \mathrm{O}$ & -4.32 & -4.33 & -4.3 & -4.26 & -3.91 \\
\hline Halite & $\mathrm{NaCl}$ & -- & -- & -10.2 & -10.1 & -10.05 \\
\hline Hausmannite & $\mathrm{Mn}_{3} \mathrm{O}_{4}$ & -34.1 & -33.78 & -30.27 & -30.79 & -35.08 \\
\hline Hematite & $\mathrm{Fe}_{2} \mathrm{O}_{3}$ & 2.87 & 3.99 & 6.76 & 6.65 & 2.42 \\
\hline Manganite & $\mathrm{MnOOH}$ & -12.17 & -12.1 & -11.35 & -11.6 & -12.74 \\
\hline Melanterite & $\mathrm{FeSO}_{4}: 7 \mathrm{H}_{2} \mathrm{O}$ & -7.38 & -6.85 & -6.59 & -6.36 & -6.84 \\
\hline Pyrochroite & $\mathrm{Mn}(\mathrm{OH})_{2}$ & -11 & -10.93 & -10.42 & -10.57 & -11.32 \\
\hline Pyrolusite & $\mathrm{MnO}_{2}: \mathrm{H}_{2} \mathrm{O}$ & -21.58 & -21.43 & -19.47 & -19.75 & -22.08 \\
\hline Quartz & $\mathrm{SiO}_{2}$ & -0.07 & -0.08 & -0.09 & -0.11 & 0.02 \\
\hline Siderite & $\mathrm{FeCO}_{3}$ & -4.31 & -2.89 & -3.2 & -2.86 & -3.99 \\
\hline $\mathrm{SiO} 2(\mathrm{a})$ & $\mathrm{SiO}_{2}$ & -1.43 & -1.44 & -1.41 & -1.43 & -1.33 \\
\hline Strontianite & $\mathrm{SrCO}_{3}$ & -- & -- & -- & -- & -- \\
\hline Witherite & $\mathrm{BaCO}_{3}$ & -7.81 & -6.78 & -7.43 & -7.49 & -8.05 \\
\hline
\end{tabular}


Table 9. HLDTC Mineral Saturation Indexes (SI) (cont.)

\begin{tabular}{|c|c|c|c|c|c|c|}
\hline & Sample ID & \multicolumn{5}{|c|}{ MW-9R } \\
\hline & Quarter Date & $1 / 7 / 2017$ & $4 / 9 / 2017$ & $7 / 8 / 2017$ & $10 / 26 / 2017$ & $1 / 4 / 2018$ \\
\hline Anhydrite & $\mathrm{CaSO}_{4}$ & -4.15 & -4 & -3.93 & -3.9 & -4.01 \\
\hline Barite & $\mathrm{BaSO}_{4}$ & -0.3 & -0.85 & -0.89 & -0.81 & -0.89 \\
\hline Calcite & $\mathrm{CaCO}_{3}$ & -2.2 & -2.39 & -2.08 & -2.55 & -0.77 \\
\hline Celestite & $\mathrm{SrSO}_{4}$ & -3.82 & -3.72 & -3.68 & -3.59 & -3.7 \\
\hline $\mathrm{CO} 2(\mathrm{~g})$ & $\mathrm{CO}_{2}$ & -1.68 & -1.52 & -1.59 & -1.38 & -3.14 \\
\hline Dolomite & $\mathrm{CaMg}(\mathrm{CO} 3)_{2}$ & -4.35 & -4.72 & -4.04 & -4.99 & -1.53 \\
\hline $\mathrm{Fe}(\mathrm{OH}) 3(\mathrm{a})$ & $\mathrm{Fe}(\mathrm{OH})_{3}$ & 1.29 & 0.17 & 0.66 & -0.11 & 3.79 \\
\hline Fluorite & $\mathrm{CaF}_{2}$ & -- & -- & -3.35 & -3.24 & -3.36 \\
\hline Goethite & $\mathrm{FeOOH}$ & 6.69 & 5.61 & 6.19 & 5.45 & 9.2 \\
\hline Gypsum & $\mathrm{CaSO}_{4}: 2 \mathrm{H}_{2} \mathrm{O}$ & -3.69 & -3.56 & -3.51 & -3.5 & -3.55 \\
\hline Halite & $\mathrm{NaCl}$ & -9.1 & -9.18 & -9.29 & -9.34 & -9.44 \\
\hline Hausmannite & $\mathrm{Mn}_{3} \mathrm{O}_{4}$ & -17.43 & -19.22 & -17.69 & -20.11 & -6.88 \\
\hline Hematite & $\mathrm{Fe}_{2} \mathrm{O}_{3}$ & 15.33 & 13.17 & 14.34 & 12.87 & 20.35 \\
\hline Manganite & $\mathrm{MnOOH}$ & -6.12 & -6.89 & -6.55 & -7.53 & -2.19 \\
\hline Melanterite & $\mathrm{FeSO}_{4}: 7 \mathrm{H}_{2} \mathrm{O}$ & -6.28 & -6.7 & -6.76 & -6.5 & -7.89 \\
\hline Pyrochroite & $\mathrm{Mn}(\mathrm{OH})_{2}$ & -6.62 & -7.14 & -6.95 & -7.58 & -4.03 \\
\hline Pyrolusite & $\mathrm{MnO}_{2}: \mathrm{H}_{2} \mathrm{O}$ & -13.74 & -14.58 & -13.66 & -14.88 & -8.39 \\
\hline Quartz & $\mathrm{SiO}_{2}$ & 0.3 & 0.36 & 0.4 & 0.41 & 0.43 \\
\hline Siderite & $\mathrm{FeCO}_{3}$ & -0.19 & -0.91 & -0.68 & -0.9 & -0.5 \\
\hline $\mathrm{SiO} 2(\mathrm{a})$ & $\mathrm{SiO}_{2}$ & -1.06 & -0.99 & -0.93 & -0.92 & -0.92 \\
\hline Strontianite & $\mathrm{SrCO}_{3}$ & -3.46 & -3.7 & -3.42 & -3.83 & -2.05 \\
\hline \multirow[t]{3}{*}{ Witherite } & $\mathrm{BaCO}_{3}$ & -- & -- & -- & -5.05 & -- \\
\hline & Sample ID & & & MW-10R & & \\
\hline & Quarter Date & $1 / 8 / 2017$ & $4 / 8 / 2017$ & $7 / 9 / 2017$ & $10 / 27 / 2017$ & $1 / 1 / 2018$ \\
\hline Anhydrite & $\mathrm{CaSO}_{4}$ & -- & -- & -- & -- & -- \\
\hline Barite & $\mathrm{BaSO}_{4}$ & -- & -- & -- & -- & -- \\
\hline Calcite & $\mathrm{CaCO}_{3}$ & -5.25 & -5.87 & -6.03 & -6.99 & -6.21 \\
\hline Celestite & $\mathrm{SrSO}_{4}$ & -- & -- & -- & -- & -- \\
\hline $\mathrm{CO} 2(\mathrm{~g})$ & $\mathrm{CO}_{2}$ & -1.43 & -1.32 & -1.32 & -1.74 & -0.81 \\
\hline Dolomite & $\mathrm{CaMg}(\mathrm{CO} 3)_{2}$ & -10.32 & -11.3 & -11.38 & -13.25 & -11.98 \\
\hline $\mathrm{Fe}(\mathrm{OH}) 3(\mathrm{a})$ & $\mathrm{Fe}(\mathrm{OH})_{3}$ & -3.67 & -4.14 & -5.46 & -4.53 & -5.24 \\
\hline Fluorite & $\mathrm{CaF}_{2}$ & -- & -- & -- & -- & -- \\
\hline Goethite & $\mathrm{FeOOH}$ & 1.71 & 1.34 & 0.07 & 0.99 & 0.17 \\
\hline Gypsum & $\mathrm{CaSO}_{4}: 2 \mathrm{H}_{2} \mathrm{O}$ & -- & -- & -- & -- & -- \\
\hline Halite & $\mathrm{NaCl}$ & -9.97 & -10.04 & -10.05 & -10.03 & -10.03 \\
\hline Hausmannite & $\mathrm{Mn}_{3} \mathrm{O}_{4}$ & -30.02 & -30.07 & -30.09 & -32.3 & -34.16 \\
\hline Hematite & $\mathrm{Fe}_{2} \mathrm{O}_{3}$ & 5.37 & 4.63 & 2.1 & 3.93 & 2.28 \\
\hline Manganite & $\mathrm{MnOOH}$ & -10.68 & -11.02 & -11.13 & -11.93 & -12.33 \\
\hline Melanterite & $\mathrm{FeSO}_{4}: 7 \mathrm{H}_{2} \mathrm{O}$ & -- & -- & -- & -- & \\
\hline Pyrochroite & $\mathrm{Mn}(\mathrm{OH})_{2}$ & -9.97 & -10.04 & -10.15 & -10.73 & -10.95 \\
\hline Pyrolusite & $\mathrm{MnO}_{2}: \mathrm{H}_{2} \mathrm{O}$ & -19.58 & -19.73 & -19.65 & -20.69 & -21.81 \\
\hline Quartz & $\mathrm{SiO}_{2}$ & 0.1 & 0.04 & 0.06 & 0.03 & 0.12 \\
\hline Siderite & $\mathrm{FeCO}_{3}$ & -3.69 & -3.81 & -5.14 & -4.42 & -3.97 \\
\hline $\mathrm{SiO} 2(\mathrm{a})$ & $\mathrm{SiO}_{2}$ & -1.26 & -1.3 & -1.28 & -1.31 & -1.24 \\
\hline Strontianite & $\mathrm{SrCO}_{3}$ & -- & -- & -- & -- & -- \\
\hline Witherite & $\mathrm{BaCO}_{3}$ & -7.33 & -7.58 & -7.06 & -8.62 & -8.05 \\
\hline
\end{tabular}


Table 9. HLDTC Mineral Saturation Indexes (SI) (cont.)

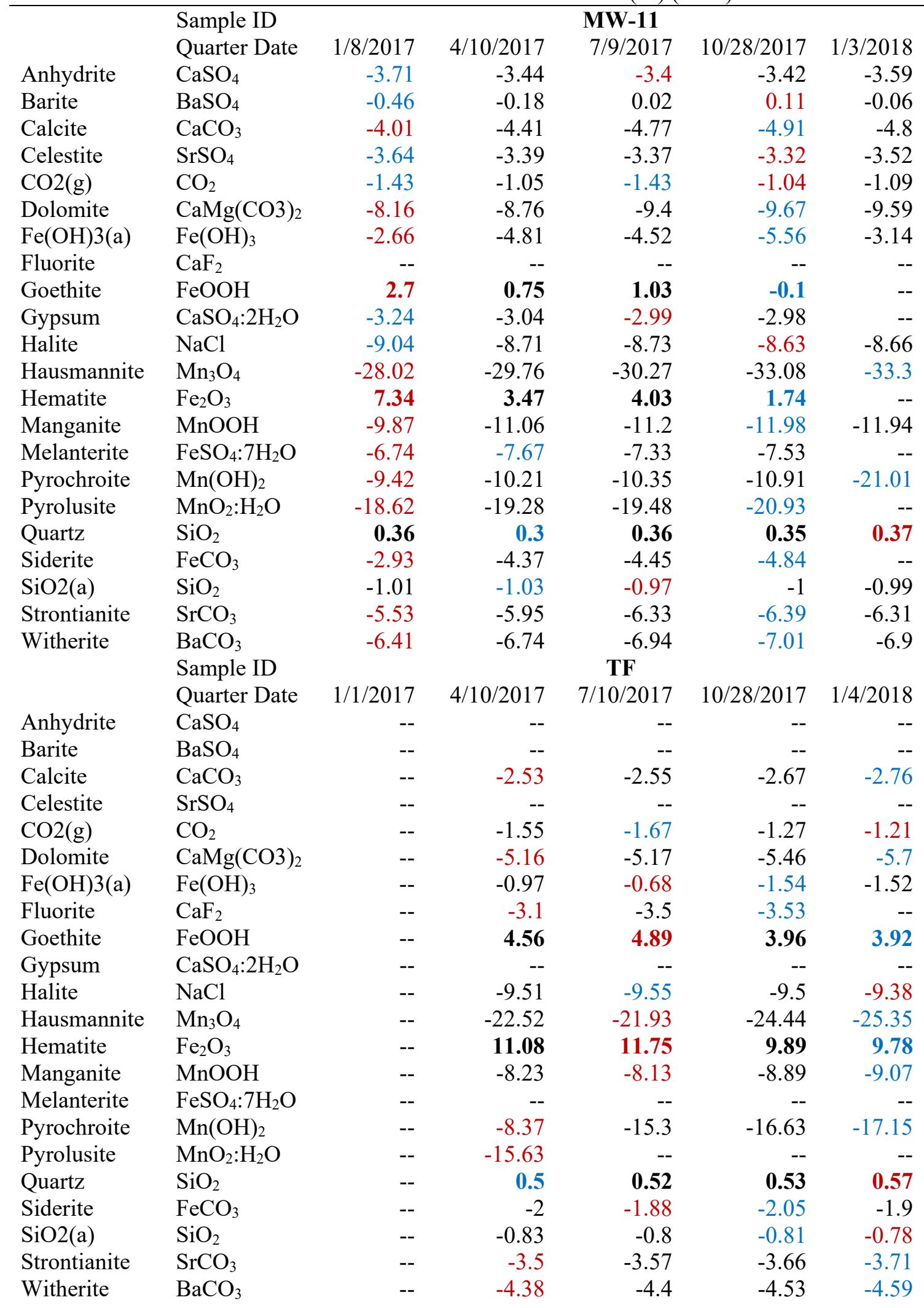


Table 9. HLDTC Mineral Saturation Indexes (SI) (cont.)

\begin{tabular}{|c|c|c|c|c|c|c|}
\hline & Sample ID & & & CR & & \\
\hline & Quarter Date & $1 / 8 / 2017$ & $4 / 10 / 2017$ & $7 / 12 / 2017$ & $10 / 25 / 2017$ & $1 / 1 / 2018$ \\
\hline Anhydrite & $\mathrm{CaSO}_{4}$ & -2.57 & -2.57 & -2.22 & -2.51 & -2.68 \\
\hline Barite & $\mathrm{BaSO}_{4}$ & -0.02 & -0.2 & -0.16 & -0.21 & -0.12 \\
\hline Calcite & $\mathrm{CaCO}_{3}$ & 0.71 & -0.73 & -0.03 & -2.88 & 0.39 \\
\hline Celestite & $\mathrm{SrSO}_{4}$ & -2.54 & -2.62 & -2.35 & -2.52 & -2.68 \\
\hline $\mathrm{CO} 2(\mathrm{~g})$ & $\mathrm{CO}_{2}$ & -4.52 & 1.3 & -2.69 & -5.97 & -4.29 \\
\hline Dolomite & $\mathrm{CaMg}(\mathrm{CO} 3)_{2}$ & 1.06 & -64.37 & 0.01 & -5.93 & 0.36 \\
\hline $\mathrm{Fe}(\mathrm{OH}) 3(\mathrm{a})$ & $\mathrm{Fe}(\mathrm{OH})_{3}$ & -- & -- & -- & 1.85 & 2.88 \\
\hline Fluorite & $\mathrm{CaF}_{2}$ & -- & -- & -2.91 & -2.89 & -2.46 \\
\hline Goethite & $\mathrm{FeOOH}$ & -- & 6.78 & -- & 7.33 & 7.83 \\
\hline Gypsum & $\mathrm{CaSO}_{4}: 2 \mathrm{H}_{2} \mathrm{O}$ & -1.99 & -2.14 & -1.92 & -2.07 & -2.1 \\
\hline Halite & $\mathrm{NaCl}$ & -8.52 & -8.9 & -8.45 & -8.48 & -8.64 \\
\hline Hausmannite & $\mathrm{Mn}_{3} \mathrm{O}_{4}$ & -5.89 & -15.79 & -10.96 & -11.79 & -7.74 \\
\hline Hematite & $\mathrm{Fe}_{2} \mathrm{O}_{3}$ & -- & 15.51 & -- & 16.61 & 17.55 \\
\hline Manganite & $\mathrm{MnOOH}$ & -0.44 & -5.43 & -4.71 & -3.93 & -1.07 \\
\hline Melanterite & $\mathrm{FeSO}_{4}: 7 \mathrm{H}_{2} \mathrm{O}$ & -- & -8.33 & -- & -9.25 & -10.31 \\
\hline Pyrochroite & $\mathrm{Mn}(\mathrm{OH})_{2}$ & -3.56 & -6.91 & -6.37 & -5.9 & -3.9 \\
\hline Pyrolusite & $\mathrm{MnO}_{2}: \mathrm{H}_{2} \mathrm{O}$ & -7.3 & -11.7 & -8.96 & -9.72 & -8.38 \\
\hline Quartz & $\mathrm{SiO}_{2}$ & -0.09 & -1.6 & -0.47 & -0.26 & -0.03 \\
\hline Siderite & $\mathrm{FeCO}_{3}$ & -- & -- & -- & -5.42 & -3.41 \\
\hline $\mathrm{SiO} 2(\mathrm{a})$ & $\mathrm{SiO}_{2}$ & -1.52 & -- & -1.74 & -1.61 & -1.47 \\
\hline Strontianite & $\mathrm{SrCO}_{3}$ & -0.81 & -2.37 & -1.74 & -4.48 & -1.15 \\
\hline Witherite & $\mathrm{BaCO}_{3}$ & -2.46 & -2.3 & -3.45 & -6.19 & -2.77 \\
\hline Notes: & & & & & & \\
\hline & $\begin{array}{l}-- \\
\text { red } \\
\text { blue }\end{array}$ & $\begin{array}{l}\text { No data } \\
\text { maximun } \\
\text { minimum }\end{array}$ & $\begin{array}{l}\text { value } \\
\text { value }\end{array}$ & & & \\
\hline
\end{tabular}

Although mineralogical analyses were not performed on core samples in this study, Weinheimer (1983) performed sedimentological and mineralogical analyses of thin sections from four cores in the Breathitt Fm. in eastern Kentucky. Average mineral percentages were as follows: quartz 47\%; feldspar (mainly K-feldspar) 29\%; rock fragments $11.9 \%$; mica 5.4\%; and heavy (accessory) minerals (pyrite and siderite) $0.5 \%$. The cement was mostly ferroan calcite; authigenic kaolinite filled pore spaces and formed reaction rims around feldspar grains. Detrital organic matter, iron oxides, and (rarely) dolomite were also detected. Shales and claystones contained illite, kaolinite, and chlorite. The phases other than quartz, which is relatively resistant to weathering, may regulate 
groundwater chemistry, but, as noted above, SI values were not determined for aluminosilicates and pyrite in this study.

The Norton Fm. of Virginia is described as being very similar to the Breathitt Fm. in Kentucky. From their study of the Norton Fm., Powell and Larson (1985) related groundwater chemistry to mineralogy within coal-bearing strata, proposing a series of chemical reactions to account for the observed water types. Wunsch (1993) studied groundwater geochemistry at an unmined site in Perry County, Kentucky, $\sim 80 \mathrm{~km}$ northwest of HLDTC within the coal-bearing strata of the Breathitt Fm. Wunsch (1993) noted that the chemical reactions proposed by Powell and Larson (1985) were applicable to the site and supported the observed mineralogy and water types. Four of the 16 piezometers, installed amongst eight wells, at Wunsch's study site exhibited more acidic values that reflect the approximate site-wide 6.1 mean $\mathrm{pH}$ at HLDTC. The piezometers' water chemistry was also similar with regards to reported major anion and cation concentrations. According to Wunsch, the water type present was due to dissolution of calcite with concomitant oxidation of pyrite, indicated by low $\mathrm{pH}$ values and high sulfate and high iron content. The relevant reactions include calcite dissolution:

$$
\mathrm{CaCO}_{3}+\mathrm{H}_{2} \mathrm{CO}_{3} \rightarrow \mathrm{Ca}^{2+}+2 \mathrm{HCO}_{3}^{-}
$$

and pyrite oxidation to form Fe (oxyhydr)oxides:

$$
\begin{gathered}
\mathrm{FeS}_{2}+3.5 \mathrm{O}_{2}+\mathrm{H}_{2} \mathrm{O} \rightarrow \mathrm{Fe}^{2+}+2 \mathrm{SO}_{4}^{2-}+2 \mathrm{H}^{+} \\
4 \mathrm{Fe}^{2+}+\mathrm{O}_{2}+4 \mathrm{H}^{+}=4 \mathrm{Fe}^{3+}+2 \mathrm{H}_{2} \mathrm{O} \\
\mathrm{Fe}^{3+}+3 \mathrm{H}_{2} \mathrm{O}=\mathrm{Fe}(\mathrm{OH})_{3(\mathrm{~s})}+3 \mathrm{H}^{+} \\
\mathrm{FeS}_{2}+14 \mathrm{Fe}^{3+}+8 \mathrm{H}_{2} \mathrm{O} \rightarrow 15 \mathrm{Fe}^{3+}+2 \mathrm{SO}_{4}^{2-}+16 \mathrm{H}^{+}
\end{gathered}
$$


Sulfate can be also be reduced to hydrogen sulfide by anaerobic bacteria under anoxic conditions:

$$
2 \mathrm{CH}_{2} \mathrm{O}+\mathrm{SO}_{4}^{2-} \leftrightarrow \mathrm{HCO}_{3}^{-}+\mathrm{HS}^{-}+\mathrm{CO}_{2}+\mathrm{H}_{2}
$$

The occurrence of magnesium may be related to reaction with chlorite, an abundant mineral in coal-field rocks:

$$
\begin{aligned}
& \mathrm{Mg}_{3}(\mathrm{OH})_{6}\left(\mathrm{Mg}_{2} \mathrm{Al}\right)\left(\mathrm{AlSi}_{3}\right) \mathrm{O}_{10}(\mathrm{OH})_{2}+6 \mathrm{H}^{+}+\mathrm{HCO}_{3}^{-} \\
& =3 \mathrm{Mg}^{2+}+6 \mathrm{H}_{2} \mathrm{O}+6 \mathrm{HCO}_{3}^{-}+\left(\mathrm{Mg}_{2} \mathrm{Al}\right)\left(\mathrm{AlSi}_{3}\right) \mathrm{O}_{10}(\mathrm{OH})_{2}
\end{aligned}
$$

Results from water samples collected in April and October 2017 for volatile organic compounds (VOCs) are summarized in Table 10. The laboratory analysis reports for quarters two and four are included in the appendices as McCoy Quarter No. VOC Analytical Report. 2-butanone and 2-hexanone were detected in MW-5 in October 2017 at an estimated value of $4 \mu \mathrm{g} / \mathrm{L}$ for both analytes and in the field blank in April 2017 at 9 $\mu \mathrm{g} / \mathrm{L}$ for 2-butanone and an estimated $2 \mathrm{ug} / \mathrm{L}$ for 2 -hexanone. Acetone was detected in most water samples for April and October 2017 (range 2-57 $\mu \mathrm{g} / \mathrm{L}$, mean $16.5 \mu \mathrm{g} / \mathrm{L}$ ) and in the field blank in April $2017(13 \mu \mathrm{g} / \mathrm{L})$. All three of these analytes are common laboratory contaminants. Chloromethane was not detected $(<2 \mu \mathrm{g} / \mathrm{L})$ in the April 2017 samples, but was detected (range $2-5 \mu \mathrm{g} / \mathrm{L}$, mean $2.9 \mu \mathrm{g} / \mathrm{L}$ ) in the October 2017 samples. Low levels of chloromethane occur naturally in the environment as a byproduct of biomass degradation associated with undeveloped land, and chloromethane has been correlated with septic system density, anoxic water conditions, and silty soils (Zogorski et al., 2006). Tetrahydrofuran (THF) was detected in the October 2017 samples from MW-2 (3 $\mu \mathrm{g} / \mathrm{L})$ and the Cumberland River $(9 \mu \mathrm{g} / \mathrm{L})$. The result from MW-2 is suspect due to the potential for improper well development. THF is used in manufacturing PVC pipe and commonly 
in adhesives. The potential for leaching THF from PVC well casing is well documented (Lapham et al., 1995). The source of THF in the Cumberland River is unknown.

The decision to conduct sampling for VOCs was dictated in part by the presence of 14 onsite natural gas wells, six of which are in production (Figure 3). Contamination of overlying, shallow bodies of water by hydrocarbons (e.g., benzene, toluene, ethylbenzene, and isomers of xylene) can occur via upward migration due to improperly cased or deteriorated casing, plugged or abandoned gas wells or during the drilling process (Price et al., 1962). An additional concern exists for saltwater contamination when gas wells penetrate the saltwater interface. Hopkins (1966) mapped the fresh-saline water interface for Kentucky. Based upon the map, the interface at HLDTC occurs at $\sim 244 \mathrm{~m}$ asl, land surface elevation varies from 378 to $427 \mathrm{~m}$ asl, and onsite gas wells are drilled to depths of 322 to $687 \mathrm{~m}$ bls. Therefore, onsite gas wells are likely completed below the fresh-saline water interface. The monitoring well water-quality parameters (Table 3) and chemical analysis results (Tables 4-10) do not indicate either hydrocarbon or brine migration upward into the surficial aquifer. 
Table 10. HLDTC VOC Results for Detected Analytes

\begin{tabular}{|c|c|c|c|c|c|}
\hline Quarter Date & 2-Butanone & 2-Hexanone & Acetone & $\begin{array}{l}\text { Chloro- } \\
\text { methane }\end{array}$ & $\begin{array}{l}\text { Tetra- } \\
\text { hydrofuran }\end{array}$ \\
\hline MDL (ug/L) & 2 & 2 & 2 & 2 & 2 \\
\hline $\begin{array}{l}\text { NPDWRs - MCL } \\
(\mathrm{mg} / \mathrm{L})\end{array}$ & $\mathrm{n} / \mathrm{a}$ & $\mathrm{n} / \mathrm{a}$ & $\mathrm{n} / \mathrm{a}$ & $\mathrm{n} / \mathrm{a}$ & $\mathrm{n} / \mathrm{a}$ \\
\hline $\begin{array}{l}\text { NSDWRs - SMCL } \\
(\mathrm{mg} / \mathrm{L})\end{array}$ & $\mathrm{n} / \mathrm{a}$ & $\mathrm{n} / \mathrm{a}$ & $\mathrm{n} / \mathrm{a}$ & $\mathrm{n} / \mathrm{a}$ & $\mathrm{n} / \mathrm{a}$ \\
\hline Sample ID & \multicolumn{5}{|c|}{ MW-1 } \\
\hline $4 / 7 / 2017$ & $<\mathrm{MDL}$ & $<\mathrm{MDL}$ & $3 \mathrm{~J}$ & $<\mathrm{MDL}$ & $<\mathrm{MDL}$ \\
\hline $10 / 26 / 2017$ & $<\mathrm{MDL}$ & $<\mathrm{MDL}$ & 21 & $3 \mathrm{~J}$ & $<\mathrm{MDL}$ \\
\hline Sample ID & \multicolumn{5}{|c|}{ MW-2 } \\
\hline $4 / 8 / 2017$ & $<\mathrm{MDL}$ & $<\mathrm{MDL}$ & 19 & $<\mathrm{MDL}$ & $<\mathrm{MDL}$ \\
\hline $10 / 26 / 2017$ & $<\mathrm{MDL}$ & $<\mathrm{MDL}$ & 47 & $4 \mathrm{~J}$ & $3 \mathrm{~J}$ \\
\hline Sample ID & \multicolumn{5}{|c|}{ MW-3R } \\
\hline $4 / 7 / 2017$ & $<\mathrm{MDL}$ & $<\mathrm{MDL}$ & 6 & $<\mathrm{MDL}$ & $<\mathrm{MDL}$ \\
\hline $10 / 26 / 2017$ & $<\mathrm{MDL}$ & $<\mathrm{MDL}$ & $4 \mathrm{~J}$ & 5 & $<\mathrm{MDL}$ \\
\hline Sample ID & \multicolumn{5}{|c|}{ MW-4 } \\
\hline $4 / 7 / 2017$ & $<\mathrm{MDL}$ & $<\mathrm{MDL}$ & 32 & $<\mathrm{MDL}$ & $<\mathrm{MDL}$ \\
\hline $10 / 28 / 2017$ & $<\mathrm{MDL}$ & $<\mathrm{MDL}$ & 15 & $<\mathrm{MDL}$ & $<\mathrm{MDL}$ \\
\hline $10 / 28 / 2017$ & & & & & \\
\hline (duplicate) & $<\mathrm{MDL}$ & $<\mathrm{MDL}$ & $<\mathrm{MDL}$ & $2 \mathrm{~J}$ & $<\mathrm{MDL}$ \\
\hline Sample ID & \multicolumn{5}{|c|}{ MW-5 } \\
\hline $4 / 9 / 2017$ & $<\mathrm{MDL}$ & $<\mathrm{MDL}$ & 15 & $<\mathrm{MDL}$ & $<\mathrm{MDL}$ \\
\hline $10 / 27 / 2017$ & 4J & $4 \mathrm{~J}$ & $4 \mathrm{~J}$ & $2 \mathrm{~J}$ & $<\mathrm{MDL}$ \\
\hline Sample ID & \multicolumn{5}{|c|}{ MW-6 } \\
\hline $4 / 9 / 2017$ & $<\mathrm{MDL}$ & $<\mathrm{MDL}$ & 7 & $<\mathrm{MDL}$ & $<\mathrm{MDL}$ \\
\hline $4 / 9 / 2017$ & & & & & \\
\hline (duplicate) & $<\mathrm{MDL}$ & $<\mathrm{MDL}$ & $<\mathrm{MDL}$ & $<\mathrm{MDL}$ & $<\mathrm{MDL}$ \\
\hline $10 / 27 / 2017$ & $<\mathrm{MDL}$ & $<\mathrm{MDL}$ & $2 \mathrm{~J}$ & $2 \mathrm{~J}$ & $<\mathrm{MDL}$ \\
\hline Sample ID & \multicolumn{5}{|c|}{ MW-7 } \\
\hline $4 / 9 / 2017$ & $<\mathrm{MDL}$ & $<\mathrm{MDL}$ & 9 & $<\mathrm{MDL}$ & $<\mathrm{MDL}$ \\
\hline $10 / 27 / 2017$ & $<\mathrm{MDL}$ & $<\mathrm{MDL}$ & 6 & $3 \mathrm{~J}$ & $<\mathrm{MDL}$ \\
\hline Sample ID & \multicolumn{5}{|c|}{ MW-8 } \\
\hline $4 / 8 / 2017$ & $<\mathrm{MDL}$ & $<\mathrm{MDL}$ & 10 & $<\mathrm{MDL}$ & $<\mathrm{MDL}$ \\
\hline $10 / 27 / 2017$ & $<\mathrm{MDL}$ & $<\mathrm{MDL}$ & $3 \mathrm{~J}$ & $2 \mathrm{~J}$ & $<\mathrm{MDL}$ \\
\hline Sample ID & \multicolumn{5}{|c|}{ MW-9R } \\
\hline $4 / 9 / 2017$ & $<\mathrm{MDL}$ & $<\mathrm{MDL}$ & 28 & $<\mathrm{MDL}$ & $<\mathrm{MDL}$ \\
\hline $10 / 26 / 2017$ & $<\mathrm{MDL}$ & $<\mathrm{MDL}$ & $4 \mathrm{~J}$ & $4 \mathrm{~J}$ & $<\mathrm{MDL}$ \\
\hline Sample ID & \multicolumn{5}{|c|}{ MW-10R } \\
\hline $4 / 8 / 2017$ & $<\mathrm{MDL}$ & $<\mathrm{MDL}$ & 11 & $<\mathrm{MDL}$ & $<\mathrm{MDL}$ \\
\hline $10 / 27 / 2017$ & $<\mathrm{MDL}$ & $<\mathrm{MDL}$ & 5 & $2 \mathrm{~J}$ & $<\mathrm{MDL}$ \\
\hline Sample ID & \multicolumn{5}{|c|}{ MW-11 } \\
\hline $4 / 10 / 2017$ & $<\mathrm{MDL}$ & $<\mathrm{MDL}$ & 25 & $<\mathrm{MDL}$ & $<\mathrm{MDL}$ \\
\hline $10 / 28 / 2017$ & $<\mathrm{MDL}$ & $<\mathrm{MDL}$ & 13 & $2 \mathrm{~J}$ & $<\mathrm{MDL}$ \\
\hline
\end{tabular}


Table 10. HLDTC VOC Results for Detected Analytes (cont.)

\begin{tabular}{|c|c|c|c|c|c|}
\hline \multirow{2}{*}{ Sample ID } & \multicolumn{5}{|c|}{ TF } \\
\hline & $<\mathrm{MDL}$ & $<\mathrm{MDL}$ & $<\mathrm{MDL}$ & $<$ MDL & $<\mathrm{MDL}$ \\
\hline $10 / 28 / 2017$ & $<\mathrm{MDL}$ & $<\mathrm{MDL}$ & $<\mathrm{MDL}$ & $2 \mathrm{~J}$ & $<\mathrm{MDL}$ \\
\hline Sample ID & & & CR & & \\
\hline $4 / 10 / 2017$ & $<\mathrm{MDL}$ & $<\mathrm{MDL}$ & 57 & $<\mathrm{MDL}$ & $<\mathrm{MDL}$ \\
\hline $10 / 25 / 2017$ & $<\mathrm{MDL}$ & $<\mathrm{MDL}$ & 54 & 5 & 9 \\
\hline Sample ID & & & FB & & \\
\hline $4 / 10 / 2017$ & 9 & $2 \mathrm{~J}$ & 13 & $<\mathrm{MDL}$ & $<\mathrm{MDL}$ \\
\hline $10 / 28 / 2017$ & $<\mathrm{MDL}$ & $<\mathrm{MDL}$ & $<\mathrm{MDL}$ & $2 \mathrm{~J}$ & $<\mathrm{MDL}$ \\
\hline
\end{tabular}

Notes:

-- No data; $\mathrm{n} / \mathrm{a}$ (not applicable)

MDL Method Detection Limit

NPDWRs National Primary Drinking Water Regulations - MCL

NSDWRs National Secondary Drinking Water Regulations - Secondary

MCL

$\mathrm{J}$ Estimated Value

Stable isotopes can be used to delineate sources of recharge and determine groundwater flow paths (Barnes and Allison, 1988; Sharp, 2017). The quarterly water isotope data are presented in Table 11 and graphed on Figure 14. For the entire site and over the duration of sampling from January 2017 to January $2018, \delta^{18} \mathrm{O}$ ranged from -8.7 to $-4.3 \%$ (mean $-6.9 \pm 0.5 \%$ ) and $\delta^{2} \mathrm{H}$ ranged from -49.5 to $-31.5 \%$ (mean $-39.4 \pm 2.2 \%$ ). Monitoring wells located closer to the ridge and at higher elevations in the eastern portion of the site (MW-1, MW-8, MW-9R, MW-10R, MW-11) have similar mean isotopic values ( $-7.0 \pm 0.1$ to $-6.4 \pm 0.3 \%{ }_{0} \delta^{18} \mathrm{O}$ and $-38.6 \pm 0.4$ to $\left.-35.8 \pm 0.7 \%{ }^{2} \mathrm{H}\right)$. The monitoring wells located closer to the river in the western portion of the site, where the floodplain is broader (MW-2, MW-3R, MW-4, MW-5, MW-6, MW-7), have slightly lighter mean isotopic values ( $-7.2 \pm 0.3$ to $-6.8 \pm 0.3 \%$ o $\delta^{18} \mathrm{O}$ and $-41.2 \pm 1.4$ to $-39.8 \pm 1.2 \%$ o $\left.\delta^{2} \mathrm{H}\right)$. These values are similar to mean values for the nearby Tree Farm well $\left(-7.2 \pm 0.4 \%\right.$ o $\delta^{18} \mathrm{O}$ and -41.0 $\left.\pm 1.3 \% \delta^{2} \mathrm{H}\right)$ and slightly more enriched than mean values for the Cumberland River (-7.5 $\pm 0.7 \%$ o $\delta^{18} \mathrm{O}$ and $-41.4 \pm 4.3 \%$ o $\left.\delta^{2} \mathrm{H}\right)$. Although it is possible that the river is the dominant 
recharge source for alluvial groundwater (Brookfield et al., 2017), there is little evidence that bank recharge occurs outside of the riparian zone (Fetter, 1994). MW-5 and MW-7 are the only wells located within that zone, and quarterly chemical analysis does not support a connection between the other monitoring wells with lighter isotopic values and the Cumberland River. Alternatively, the slightly lighter values in the western part of the site may reflect recharge at higher elevations (Sharp, 2017). Unfortunately, the isotope spatial pattern alone does not identify a definitive recharge source or flow path, as the difference between the two groupings of wells falls within the analytical standard uncertainty.

\begin{tabular}{|c|c|c|}
\hline Quarter Date & $\begin{array}{l}\delta^{18} \mathrm{O} \\
(\% \mathrm{VSMOW})\end{array}$ & $\begin{array}{l}\delta^{2} \mathrm{H} \\
(\% \text { VSMOW) }\end{array}$ \\
\hline Sample ID & \multicolumn{2}{|c|}{ MW-1 } \\
\hline $1 / 7 / 2017$ & -6.9 & -38.7 \\
\hline $4 / 7 / 2017$ & -6.9 & -37.9 \\
\hline $7 / 8 / 2017$ & -7.0 & -38.9 \\
\hline $10 / 26 / 2017$ & -6.8 & -38.4 \\
\hline $1 / 2 / 2018$ & -6.8 & -36.6 \\
\hline mean & -6.9 & -38.1 \\
\hline st. dev. & 0.1 & 0.9 \\
\hline Sample ID & \multicolumn{2}{|c|}{ MW-2 } \\
\hline $1 / 1 / 2017$ & -6.8 & -39.5 \\
\hline $4 / 8 / 2017$ & -7.2 & -40.7 \\
\hline 7/8/2017 & -7.2 & -41.1 \\
\hline $10 / 26 / 2017$ & -7.1 & -40.5 \\
\hline $1 / 2 / 2018$ & -7.6 & -41.0 \\
\hline mean & -7.2 & -40.6 \\
\hline st. dev. & 0.3 & 0.6 \\
\hline Sample ID & \multicolumn{2}{|c|}{ MW-3R } \\
\hline $1 / 6 / 2017$ & -7.3 & -41.4 \\
\hline $4 / 7 / 2017$ & -7.3 & -41.1 \\
\hline $7 / 8 / 2017$ & -7.2 & -41.4 \\
\hline $10 / 26 / 2017$ & -7.2 & -41.6 \\
\hline $1 / 2 / 2018$ & -7.2 & -40.6 \\
\hline mean & -7.2 & -41.2 \\
\hline st. dev. & 0.1 & 0.4 \\
\hline
\end{tabular}


Table 11. HLDTC Isotope Analytical Results (cont.)

\begin{tabular}{|c|c|c|}
\hline \multicolumn{3}{|l|}{ Sample ID } \\
\hline $1 / 6 / 2017$ & -7.1 & -41.5 \\
\hline $4 / 7 / 2017$ & -6.2 & -38.5 \\
\hline $7 / 9 / 2017$ & -7.3 & -42.0 \\
\hline $10 / 28 / 2017$ & -7.4 & -42.2 \\
\hline 10/28/2017 (duplicate) & -7.5 & -41.7 \\
\hline $1 / 5 / 2018$ & -7.2 & -41.4 \\
\hline mean & -7.1 & -41.2 \\
\hline st. dev. & 0.5 & 1.4 \\
\hline Sample ID & MI & \\
\hline $1 / 6 / 2017$ & -6.3 & -37.7 \\
\hline $4 / 9 / 2017$ & -6.8 & -39.3 \\
\hline $7 / 10 / 2017$ & -7.0 & -41.0 \\
\hline $10 / 27 / 2017$ & -7.1 & -41.0 \\
\hline $1 / 4 / 2018$ & -6.9 & -40.0 \\
\hline mean & -6.8 & -39.8 \\
\hline st. dev. & 0.3 & 1.2 \\
\hline Sample ID & MI & \\
\hline $1 / 5 / 2017$ & -7.1 & -40.4 \\
\hline $4 / 9 / 2017$ & -7.2 & -41.0 \\
\hline 4/9/2017 (duplicate) & -6.5 & -38.8 \\
\hline $7 / 10 / 2017$ & -6.8 & -40.5 \\
\hline $10 / 27 / 2017$ & -7.2 & -40.8 \\
\hline $1 / 5 / 2018$ & -7.0 & -40.6 \\
\hline $1 / 5 / 2018$ (duplicate) & -7.3 & -40.8 \\
\hline mean & -7.0 & -40.4 \\
\hline st. dev. & 0.3 & 0.7 \\
\hline Sample ID & MI & \\
\hline $1 / 5 / 2017$ & -7.1 & -40.6 \\
\hline $4 / 9 / 2017$ & -6.7 & -39.4 \\
\hline $7 / 10 / 2017$ & -7.3 & -41.5 \\
\hline 7/10/2017 (duplicate) & -7.4 & -41.8 \\
\hline $10 / 27 / 2017$ & -7.5 & -41.7 \\
\hline $1 / 5 / 2018$ & -7.1 & -40.7 \\
\hline mean & -7.2 & -41.0 \\
\hline st. dev. & 0.3 & 0.9 \\
\hline Sample ID & MI & \\
\hline $1 / 7 / 2017$ & -5.9 & -35.3 \\
\hline $4 / 8 / 2017$ & -6.6 & -37.0 \\
\hline $7 / 9 / 2017$ & -6.5 & -35.5 \\
\hline $10 / 27 / 2017$ & -6.5 & -35.4 \\
\hline $1 / 3 / 2018$ & -6.4 & -35.7 \\
\hline mean & -6.4 & -35.8 \\
\hline st. dev. & 0.3 & 0.7 \\
\hline
\end{tabular}


Table 11. HLDTC Isotope Analytical Results (cont.)

\begin{tabular}{|c|c|c|}
\hline Sample ID & \multicolumn{2}{|c|}{ MW-9R } \\
\hline $1 / 7 / 2017$ & -6.6 & -36.9 \\
\hline 4/9/2017 & -6.3 & -35.8 \\
\hline $7 / 8 / 2017$ & -6.7 & -37.5 \\
\hline $10 / 26 / 2017$ & -6.8 & -37.4 \\
\hline $1 / 4 / 2018$ & -6.6 & -37.2 \\
\hline mean & -6.6 & -37.0 \\
\hline st. dev. & 0.2 & 0.7 \\
\hline Sample ID & MW & \\
\hline $1 / 8 / 2017$ & -6.9 & -37.9 \\
\hline 1/8/2017 (duplicate) & -7.1 & -39.1 \\
\hline $4 / 8 / 2017$ & -7.0 & -38.2 \\
\hline 7/9/2017 & -7.1 & -38.8 \\
\hline $10 / 27 / 2017$ & -7.1 & -38.5 \\
\hline $1 / 1 / 2018$ & -7.0 & -38.9 \\
\hline mean & -7.0 & -38.6 \\
\hline st. dev. & 0.1 & 1.4 \\
\hline Sample ID & MW & \\
\hline $1 / 8 / 2017$ & -7.0 & -40.1 \\
\hline $4 / 10 / 2017$ & -4.3 & -31.5 \\
\hline 7/9/2017 & -7.0 & -39.6 \\
\hline $10 / 28 / 2017$ & -7.6 & -41.8 \\
\hline $1 / 3 / 2018$ & -6.9 & -38.9 \\
\hline mean & -6.5 & -38.4 \\
\hline st. dev. & 1.3 & 3.6 \\
\hline Sample ID & $\mathbf{T}$ & \\
\hline $1 / 1 / 2017$ & -- & -- \\
\hline $4 / 10 / 2017$ & -6.6 & -38.9 \\
\hline $7 / 10 / 2017$ & -7.3 & -41.8 \\
\hline $10 / 28 / 2017$ & -7.6 & -42.2 \\
\hline $1 / 4 / 2018$ & -7.2 & -41.0 \\
\hline mean & -7.2 & -41.0 \\
\hline st. dev. & 0.4 & 1.3 \\
\hline Sample ID & C & \\
\hline $1 / 8 / 2017$ & -7.2 & -40.1 \\
\hline $4 / 10 / 2017$ & -7.3 & -40.8 \\
\hline $7 / 12 / 2017$ & -6.7 & -36.6 \\
\hline $10 / 25 / 2017$ & -8.7 & -49.5 \\
\hline $1 / 1 / 2018$ & -7.7 & -40.0 \\
\hline mean & -7.5 & -41.4 \\
\hline st. dev. & 0.7 & 4.3 \\
\hline
\end{tabular}

Notes:

No data 


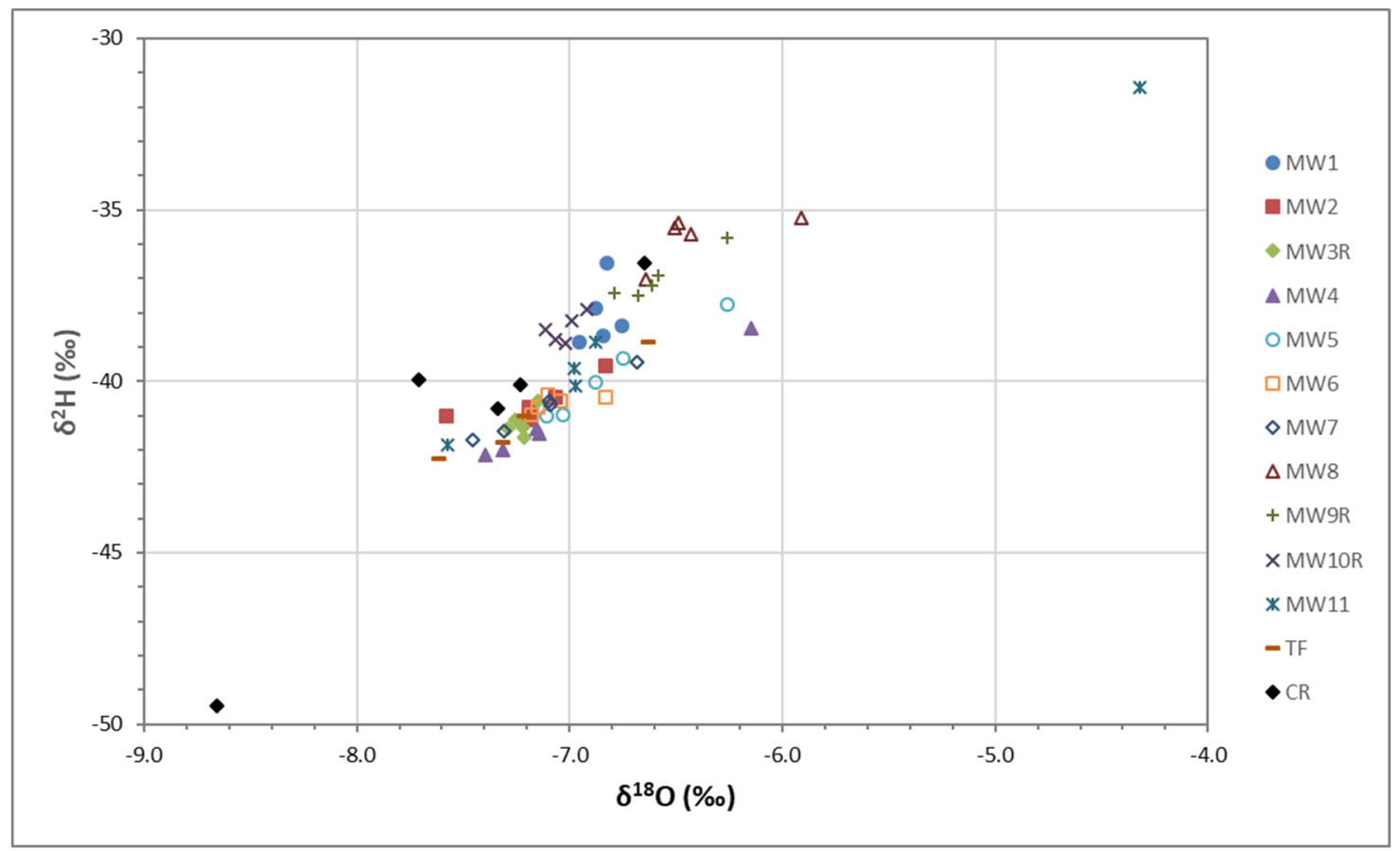

Figure 14: Stable isotopes of groundwater at HLDTC and river water from January 2017 to January 2018.

When the isotope data are grouped by quarter (January 2017, April 2017, July 2017, October 2017, January 2018) and plotted relative to the global meteoric water line (GMWL: $\delta^{2} \mathrm{H}=8 \delta^{18} \mathrm{O}+10$ ), a possible groundwater recharge pattern can be observed (Figure 15). Linear regressions for each group of quarterly samples fall subparallel to the GMWL, indicating that evaporation of the groundwater source has occurred because ${ }^{18} \mathrm{O}$ is differentially fractionated relative to ${ }^{2} \mathrm{H}$ (Gat, 1996). The steeper slopes from July and October suggest recharge from late fall through early spring, when evaporation occurs less readily. In contrast, the flatter slopes in January and April reflect more evaporated recharge from late spring to early fall. This suggests a several-month lag between infiltration of rainfall and arrival of recharge at the wells. The definitive cause of the outlier data point (MW-11) for April 2017 could not be determined; possible effects of weather (e.g., mixing with rainfall during sampling) and laboratory errors were considered and excluded. 
Therefore, it is included within the linear regression. Exclusion of the outlier modifies the line equation to $y=3.45 x-18.6\left(R^{2}=0.553\right)$. The fact that the isotope data fall to the left of the GMWL is unusual but is consistent with observations by Aseltyne et al. (2006) for shallow groundwater along Kentucky Lake in western Kentucky.

A seasonal evaluation of HLDTC isotopic data reveals slightly heavier values of $\delta^{2} \mathrm{H}$ (by $\sim 1 \%$ ) in winter and spring (January and April) and lighter values (by $\sim 0.5 \%$ ) in summer and fall (July and October). Sharp (2017) attributes this observed seasonal shift from higher values of $\delta^{2} \mathrm{H}$ in winter to lower values in summer over temperate continental regions to changes in the mean relative humidity of air masses ( $\sim 10 \%$ lower in winter). The expected timing of the greatest evaporation (late spring to early fall) and least evaporation (late fall to early spring) coincides with the highest and lowest recorded air temperatures (Figure 12). It also agrees with potential and actual evapotranspiration plots for McCracken County in western Kentucky (CH2M Hill, 1992). The inferred time lag between infiltration and recharge arrival at wells is consistent with McCoy et al. (2015) and Risser et al. (2008). 


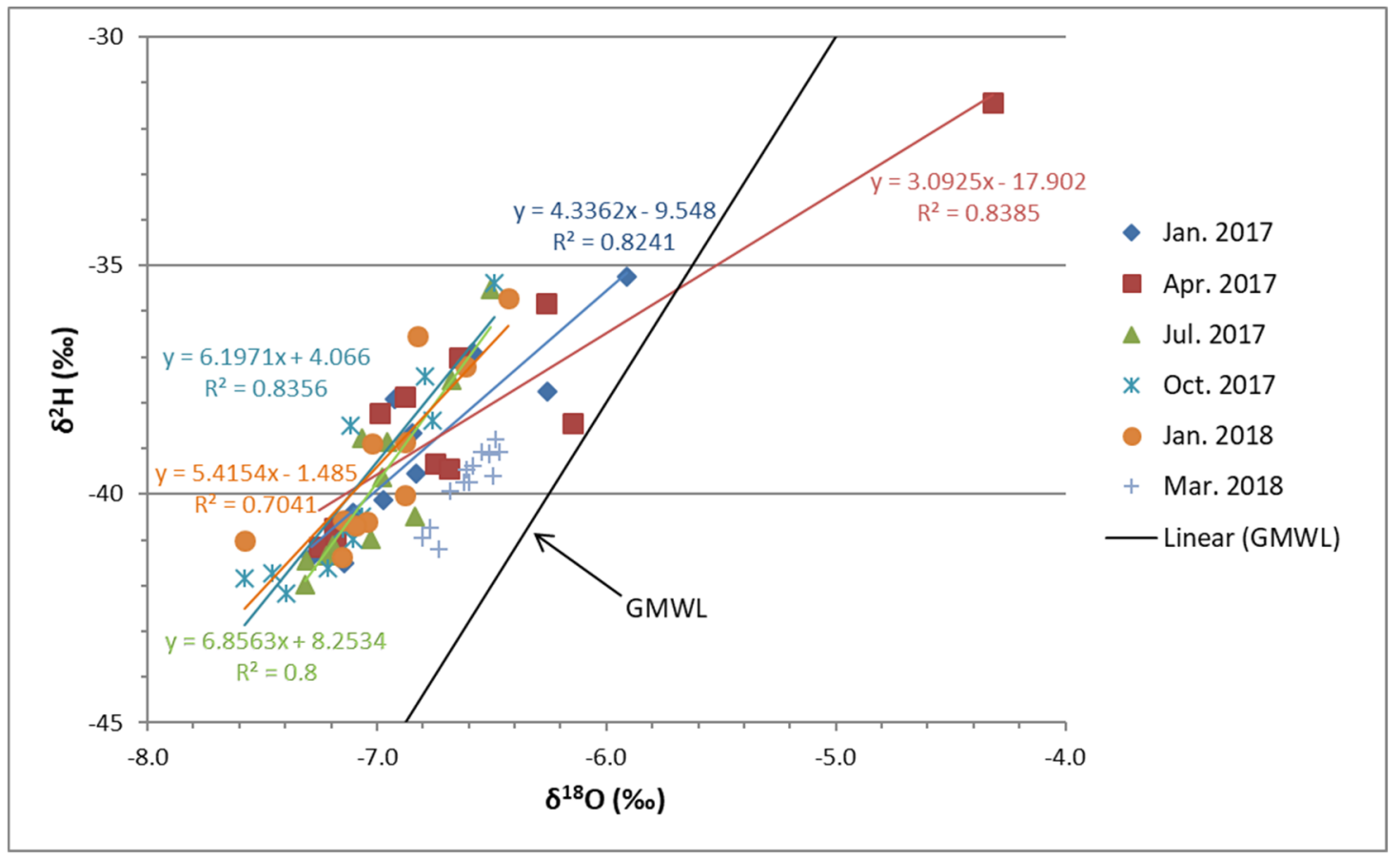

Figure 15: HLDTC quarterly monitoring-well results for $\delta^{18} \mathrm{O}$ and $\delta^{2} \mathrm{H}$ from January 2017 to March 2018.

During the pumping tests at MW-6 and MW-7 in March 2018, isotope, bromide, and chloride time-series water samples were collected (Table 12) to examine whether river water may have infiltrated during the February 2018 flood. The March 2018 isotope results fell within $\delta^{18} \mathrm{O}$ and $\delta^{2} \mathrm{H}$ ranges for those wells for January 2017, April 2017, and January 2018, which were more enriched than quarterly river samples for the same months (Figure 15). Bromide results were below MDL, which overlapped the range of values from quarterly sampling. Chloride results from MW-6 (range 1.63-4.54 mg/L, mean $3.19 \mathrm{mg} / \mathrm{L}$ ) and MW-7 (range $<1.00-1.44 \mathrm{mg} / \mathrm{L}$ ) tended to exceed previous quarterly results for MW6 (range $1.25-1.83 \mathrm{mg} / \mathrm{L}$, mean $1.45 \mathrm{mg} / \mathrm{L})$ and MW-7 (<1.00-1.03 mg/L). By comparison, chloride concentrations in quarterly river samples ranged from $2.84-5.08 \mathrm{mg} / \mathrm{L}$. The elevated chloride concentrations indicate that river water overtopped and inundated the wells, but the isotope time-series data do not agree. The time-series laboratory analytical 
reports are included in the appendices as UW Well Capacity Isotope Analytical Report and KGS Well Capacity Br-Cl Analytical Report.

Table 12. HLDTC Pumping Test Time Series Analytical Results

\begin{tabular}{|c|c|c|c|c|}
\hline Sample ID & Date \& Time & $\begin{array}{l}\delta^{18} \mathrm{O} \\
(\% \circ \mathrm{VSMOW})\end{array}$ & $\begin{array}{l}\delta^{2} \mathrm{H} \\
(\% \mathrm{~V} \text { VMOW })\end{array}$ & $\mathrm{Cl}(\mathrm{mg} / \mathrm{L})$ \\
\hline MDL & & & & 1.00 \\
\hline MW 6-1 & $3 / 11 / 2018$ 10:45 & -6.5 & -39.6 & 3.11 \\
\hline MW 6-2 & $3 / 11 / 201810: 50$ & -6.5 & -39.1 & 3.74 \\
\hline MW 6-3 & $3 / 11 / 2018$ 10:57 & -6.5 & -39.1 & 4.54 \\
\hline MW 6-4 & $3 / 11 / 2018$ 11:03 & -6.5 & -39.1 & 3.71 \\
\hline MW 6-5 & $3 / 11 / 201811: 45$ & -6.5 & -39.1 & 3.46 \\
\hline MW 6-6 & $3 / 11 / 201812: 00$ & -6.5 & -38.8 & 3.53 \\
\hline MW 6-1 & $3 / 13 / 20189: 52$ & -6.6 & -39.4 & 1.63 \\
\hline MW 6-2 & $3 / 13 / 201810: 30$ & -6.6 & -39.5 & 1.82 \\
\hline mean & & -6.5 & -39.2 & 3.19 \\
\hline st. dev. & & 0.0 & 0.3 & \\
\hline MW 7-1 & $3 / 12 / 2018$ 11:28 & -6.8 & -40.7 & 1.44 \\
\hline MW 7-2 & $3 / 12 / 2018$ 11:42 & -6.7 & -41.2 & 1.17 \\
\hline MW 7-3 & $3 / 12 / 201811: 58$ & -6.8 & -41.0 & 1.12 \\
\hline MW 7-4 & $3 / 12 / 2018$ 12:01 & -6.7 & -39.9 & 0.91 \\
\hline MW 7-1 & $3 / 13 / 201812: 03$ & -6.6 & -39.7 & $<\mathrm{MDL}$ \\
\hline MW 7-2 & $3 / 13 / 201812: 46$ & -6.6 & -39.8 & $<\mathrm{MDL}$ \\
\hline mean & & -6.7 & -40.4 & 1.16 \\
\hline st. dev. & & 0.1 & 0.7 & \\
\hline
\end{tabular}

\subsection{Site Lithology}

Based upon soil core logs, the general site lithology can be described as fining upward, where the top $\sim 0.2 \mathrm{~m}$ is silty loam, underlain by silty clay to fine sandy clay, then transitioning to clayey sands or fine to medium sands, and terminating in weathered bedrock that is either friable sandstone or shale. The eastern portion of the site is a narrow floodplain with a higher fraction of finer-grained sediments with more of the wells terminating in friable sandstone, whereas the western portion is a broad floodplain with coarser-grained fractions and terminating in friable siltstone/shale and/or sandstone. The alluvium thickens and becomes finer-grained downslope from the base of the ridge to the 
river. The appendices include hand-drawn core logs (Core Logs Handwritten), core photos (Core Photos MW No.), and digitized core logs (Core Log MW No.).

The Custom Soil Resource Report (Figure 5) concluded that most of the study area soils are silt loams, with some loam and complex (two or more soils intricately mixed) (USDA, 2017). This supports the soil core observations, where the upper portions are described as silty loam underlain by silty clay. Price et al. (1962) reported valley bottoms of tributaries to the Ohio River consist of $\sim 50 \%$ silts mixed with small quantities of sand and clay. Although the soil cores were observed to contain a high fraction of fines (silts and clays), there was also a high fraction of very fine to medium-grained sand present. Grain-size percentages were determined for a subset of cores deemed to be relatively sandy (discussed below). The sand source is likely from weathering of very fine to mediumgrained Breathitt Fm. sandstones (Price et al., 1962). Alluvial deposits in the area, which comprise the bulk of the soil cores, are described as sand, silt, clay, and gravel with a thickness of 0-4.6 m (Rice, 1974). All of these were observed except gravel. The cores terminate in the lower Breathitt Fm., for which the lithology is described as shale, siltstone, sandstone, coal, and conglomerate (Rice, 1974). Apart from the last two lithologies, this is consistent with soil core observations.

The conceptual diagram (Figure 16) depicts HLDTC's representative lithological units (composition and thickness ranges) and their relationship to monitoring-well construction details (depth ranges) and water-level ranges. The upper soil layer was loam to silty loam with a range of $0.5-1.45 \mathrm{ft}(0.15-0.44 \mathrm{~m}) \mathrm{bls}$, followed by silty clay to fine sandy clay from $0.5-4.5$ to $9.0-39.5 \mathrm{ft}(0.15-1.4$ to $2.7-12.9 \mathrm{~m}) \mathrm{bls}$, and friable shale or sandstone bedrock from $0.9-39.5$ to $14.0-44.5 \mathrm{ft}(0.27-12.9$ to $4.27-13.6 \mathrm{~m})$ bls. The water 
table ranged from 1.8-18.9 ft bls. Total well depths ranged from 14.0-44.5 ft (4.27-13.6 m) bls, with solid casing depths from 9.0-34.5 ft $(2.7-10.5 \mathrm{~m})$ bls and screen lengths from 9.0-34.5 to $14.0-44.5 \mathrm{ft}(2.7-10.5$ to $4.27-13.6 \mathrm{~m})$ bls. The water table fluctuated from being within loam to silty loam or silty clay to fine sandy clay, with the well-screen intervals ranging from silty clay to medium sand, generally falling within silty clay to fine sandy clay. 


\section{HLD Monitoring Well Construction Diagram with Representative Lithologic Units}

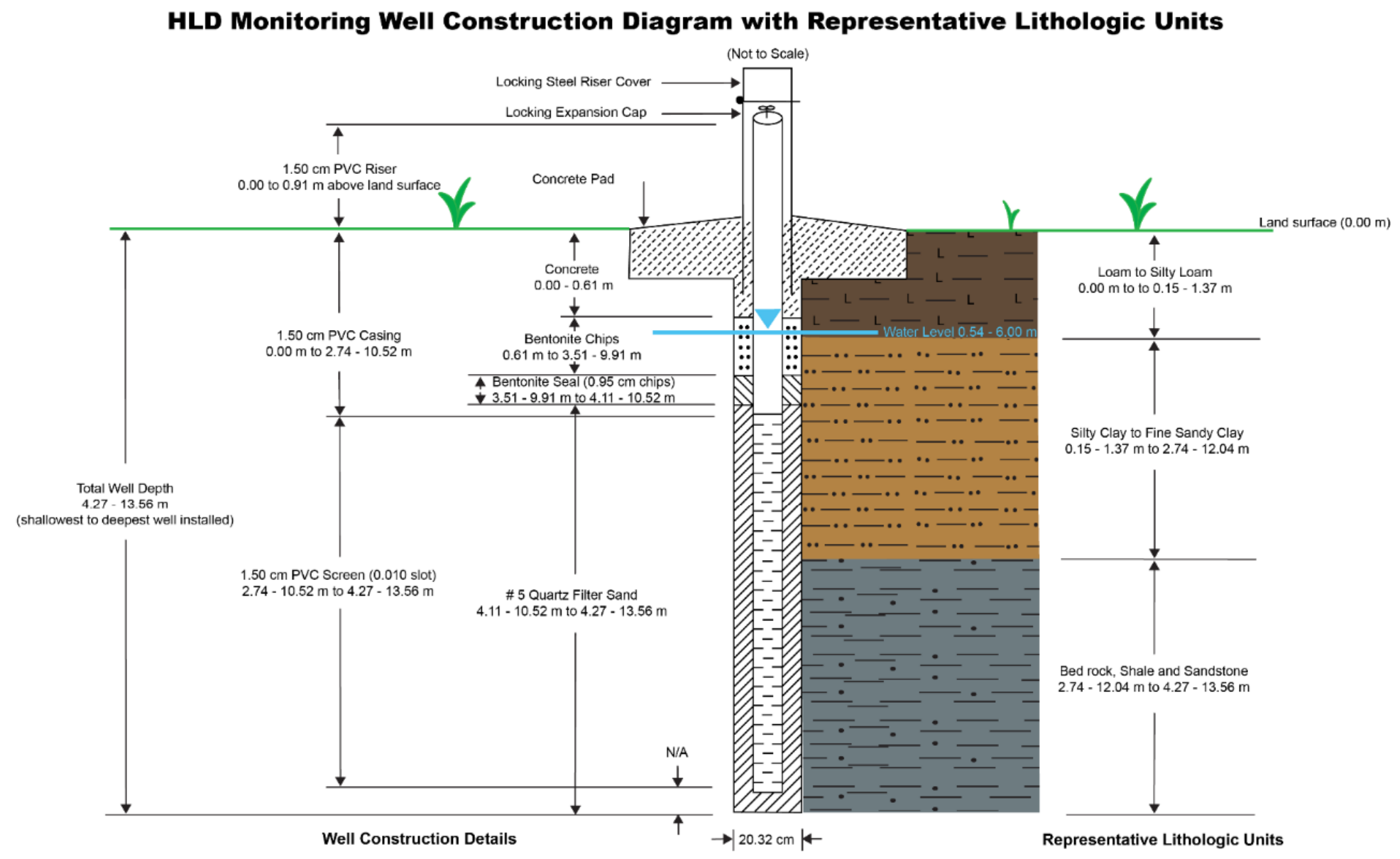

Well Construction Details: Eleven monitoring wells ranging from $4.27-13.56 \mathrm{~m}$ below land surface [bls] were installed by direct push with core collection and hollow-stem auger. Well construction consisted of drilling $1.52 \mathrm{~m}$ into bed rock Welch

Representative Litological Units: For each installed well, the top $\sim .15 \mathrm{~m}$ was composed of loam, underlain predominately by silty clay to fine sandy clay, transitioning to clayey sands or fine medium sands toward the bottom. Bedrock consisted of shale and sandstone at 3 and 8 wells, respectively.

Figure 16: HLDTC monitoring well construction diagram with representative lithologic units. 
Hydrometer and sieve analyses were conducted on a subset of samples from wells that appeared to exhibit higher fractions of sand (MW-1, MW-3R, MW-4, MW-5, MW-7, MW-8, MW-9R), as determined during core log characterization. A total of 16 samples were analyzed to determine the grain size distribution (Table 13). Sand (range 82.5397.82\%, mean 91.28\%) was the predominant size fraction, followed by clay (range 0.60 $12.00 \%$, mean $5.43 \%$, and silt $(0.59-6.70 \%$, mean $3.29 \%)$. The aforementioned size percentages are coarser than those of Price et al. (1962), who described tributary valley bottoms as consisting of 50\% silt with small quantities of sand and clay. Hydrometer and sieve data along with grain-size distribution graphs are available in the appendices as Hydrometer-Sieve Grain Size Analysis.

Table 13. HLDTC Hydrometer/Sieve Grain Size Results

\begin{tabular}{lrrrr}
\hline & & \multicolumn{3}{c}{ Proportion in class (\%) } \\
\cline { 3 - 5 } Sample ID & Depth bls (m) & \multicolumn{1}{c}{ Clay } & \multicolumn{1}{c}{ Silt } & \multicolumn{1}{c}{ Sand } \\
\hline MW-1-3 & $2.9-4.4$ & 12.00 & 3.13 & 84.87 \\
MW-3R-3 & $2.9-4.4$ & 9.00 & 1.46 & 89.54 \\
MW-3R-4 & $4.4-5.9$ & 5.80 & 1.10 & 93.10 \\
MW-4-3 & $2.9-4.4$ & 7.00 & 2.50 & 90.50 \\
MW-4-4 & $4.4-5.9$ & 2.83 & 0.59 & 96.59 \\
MW-4-5 & $5.9-7.3$ & 9.00 & 3.70 & 87.30 \\
MW-5-4 & $4.4-5.9$ & 5.00 & 3.50 & 91.50 \\
MW-5-5a & $5.9-6.6$ & 0.60 & 6.70 & 92.70 \\
MW-5-5b & $6.6-7.3$ & 3.74 & 6.13 & 90.13 \\
MW-5-6b & $7.3-8.8$ & 3.55 & 3.73 & 92.73 \\
MW-7-5 & $5.9-7.3$ & 11.00 & 6.47 & 82.53 \\
MW-7-7b & $8.8-10.2$ & 9.66 & 2.67 & 87.67 \\
MW-8-2 & $1.5-2.9$ & 1.89 & 2.55 & 95.55 \\
MW-9R-2 & $1.5-2.9$ & 2.82 & 2.82 & 94.37 \\
MW-9R-3 & $2.9-4.4$ & 1.90 & 4.55 & 93.55 \\
MW-9R-4 & $4.4-5.9$ & 1.09 & 1.09 & 97.82 \\
mean & -- & 5.43 & 3.29 & 91.28 \\
\hline
\end{tabular}

The values from the sieve analysis were entered into the program HydrogeoSieveXL, which generates a grain-size analysis report that is available in the 
appendices as HydrogeoSieveXL GrainSize MW No. All analyzed core samples are described as moderately well-sorted sand low in fines except for MW-9R-4, which is described as uniform sand low in fines. The computed total porosities are 0.38 (MW-1), mean 0.415 (MW-3R), mean 0.416 (MW-4), mean 0.408 (MW-5), mean 0.395 (MW-7), 0.42 (MW-8), and mean 0.423 (MW-9). The HydrogeoSieveXL computed porosities are similar to values of porosity ( 0.385 and 0.400$)$ from an Ohio River tributary valley bottom in eastern Kentucky (Price et al., 1962).

\subsection{Soil Chemistry}

Soil analysis was performed to determine the source of the high DOC noted in some groundwater samples (range $<0.30-17.23 \mathrm{mg} / \mathrm{L}$, mean $1.46 \mathrm{mg} / \mathrm{L}$ ) (Table 7). The results of 44 total carbon (TC) and total inorganic carbon (TIC) analyses with calculated total organic carbon (TOC) are presented in Table 14. TC ranged from $<0.0001-5.86 \%$ (mean $0.81 \%$ ) and TOC values were the same as TC, since there was no contribution from TIC. Values for 30 of 44 soil samples fell below the instrument calibration curve for low carbon analysis. The high DOC in site groundwater appears to result predominantly from decomposition of humus, which provides a source of water-soluble detrital carbon (Cronan et al., 1999; Feibig et al., 1990; Grab et al., 2012; Lyon et al., 2011), rather than of sedimentary organic matter. Additionally, although the lithology of the middle Breathitt Fm. is described as sandstone, shale, siltstone, coal, and limestone (Schweitzer, 2015), the low TIC indicates an absence of limestone within the formation at HLDTC.

Release of DOC from sediments to streams is well documented (Fiebig et al., 1990). Specifically, the riparian zone contributes substantial DOC from soils with high watersoluble detrital carbon (Cronan et al., 1999), where it concentrates in soil waters (Fiebig et 
al., 1990). Cronan et al. (1999) assessed the influences of riparian-zone land use on stream DOC. Riparian zones that were either forested or wetland contributed a mean range of 4.7$15.4 \mathrm{mg} / \mathrm{L}$ DOC to adjoining tributaries, while agricultural land use was $3.6-7.9 \mathrm{mg} / \mathrm{L}$.

The wells in this study with the highest mean DOC values were MW-5 $(1.06 \mathrm{mg} / \mathrm{L})$, MW-6 (0.63 mg/L), MW-7 (0.98 mg/L) and MW-11 (0.70 mg/L). MW-5 and MW-7 are located within the forested riparian zone, MW-11 is adjacent to the riparian zone, and MW6 is located adjacent to a constructed wetland. MW-5, 6, and 7 are adjacent to row-crop agricultural fields and MW-11 to a hay field. The elevated DOC values for these wells are consistent with the findings of Cronan et al. (1999) regarding riparian zone land use.

Table 14. HLDTC Soil Carbon Analytical Results

\begin{tabular}{|c|c|c|c|c|c|c|}
\hline Sample ID & $\begin{array}{l}\text { Depth } \\
\text { bls (m) }\end{array}$ & $\begin{array}{l}\text { TC } \\
\text { Sample } \\
\text { Weight (g) }\end{array}$ & $\begin{array}{l}\text { TIC } \\
\text { Sample } \\
\text { Weight } \\
(\mathrm{mg})\end{array}$ & TC (\%) & $\begin{array}{l}\text { TIC } \\
\text { (ppm) }\end{array}$ & $\begin{array}{l}\text { TOC } \\
(\%)\end{array}$ \\
\hline $502-630$ & -- & 0.0710 & - & 0.5220 & -- & -- \\
\hline $502-914$ & -- & 0.2135 & -- & 0.9968 & -- & -- \\
\hline $502-030$ & -- & 0.2513 & -- & 5.0989 & -- & -- \\
\hline SARM 41 & -- & 0.3513 & -- & 6.3935 & -- & -- \\
\hline$Q C$ & -- & -- & 10.5 & -- & 11.973 & -- \\
\hline MW1-1a & 0.50 & 0.3052 & 43.7 & 1.3481 & 0 & 1.3481 \\
\hline MW1-2a & 2.00 & 0.3037 & 41.9 & 0.0477 & 0 & 0.0477 \\
\hline MW1-2b & 2.51 & 0.2972 & 46.2 & 0.0183 & 0 & 0.0183 \\
\hline MW1-3a & 4.04 & 0.2923 & 40.9 & 0.0674 & 0 & 0.0674 \\
\hline MW1-3b & 4.32 & 0.3018 & 41.5 & 0.0094 & 0 & 0.0094 \\
\hline mean & 2.67 & 0.3000 & 42.9 & 0.2982 & 0 & 0.2982 \\
\hline MW2-1a & 0.05 & 0.3027 & 41.2 & 4.7513 & 0 & 4.7513 \\
\hline MW2-1b & 0.78 & 0.3092 & 45.5 & 0.1917 & 0 & 0.1917 \\
\hline $\begin{array}{l}\text { MW2-1b } \\
\text { (duplicate) }\end{array}$ & -- & - & 46.2 & -- & 0 & -- \\
\hline MW2-2a & 2.38 & 0.3007 & 44.3 & 0.1398 & 0 & 0.1398 \\
\hline$Q C$ & -- & -- & 10.8 & - & 12.103 & -- \\
\hline MW2-5a & 6.92 & 0.3073 & 42.3 & 0.0356 & 0 & 0.0356 \\
\hline mean & 2.53 & 0.3050 & 43.9 & 1.2796 & 0 & 1.2796 \\
\hline
\end{tabular}


Table 14. HLDTC Soil Carbon Analytical Results (cont.)

\begin{tabular}{|c|c|c|c|c|c|c|}
\hline MW3R-1a & 0.52 & 0.3035 & 43.1 & 1.2522 & 0 & 1.2522 \\
\hline MW3R-1b & 0.91 & 0.2989 & 40.5 & 0.6580 & 0 & 0.6580 \\
\hline$Q C$ & -- & -- & 11.0 & -- & 12.037 & -- \\
\hline MW3R-2b & 2.77 & 0.3091 & 42.6 & 0.0313 & 0 & 0.0313 \\
\hline MW3R-3a & 3.60 & 0.3067 & 41.8 & 0.0461 & 0 & 0.0461 \\
\hline MW3R-4a & 5.58 & 0.2997 & 41.7 & 0.0167 & 0 & 0.0167 \\
\hline mean & 2.676 & 0.3036 & 42.0 & 0.4009 & 0 & 0.4009 \\
\hline MW4-1a & 0.57 & 0.3021 & 42.5 & 0.3153 & 0 & 0.3153 \\
\hline MW4-2a & 2.45 & 0.3041 & 42.2 & 0.0930 & 0 & 0.0930 \\
\hline MW4-5b & 7.05 & 0.3048 & 42.3 & 0.0359 & 0 & 0.0359 \\
\hline mean & 3.36 & 0.3037 & 42.3 & 0.1481 & 0 & 0.1481 \\
\hline$Q C$ & -- & -- & 11.7 & -- & 11.770 & -- \\
\hline MW5-1a & 0.51 & 0.3056 & 48.0 & 1.9533 & 0 & 1.9533 \\
\hline MW5-2a & 1.94 & 0.3035 & 45.4 & 0.4473 & 0 & 0.4473 \\
\hline MW5-3b & 3.99 & 0.3017 & 45.6 & 0.1631 & 0 & 0.1631 \\
\hline $\begin{array}{l}\text { MW5-3b } \\
\text { (duplicate) }\end{array}$ & -- & 0.3005 & -- & 0.1800 & -- & -- \\
\hline MW5-4a & 5.75 & 0.2977 & 45.4 & 0.1903 & 0 & 0.1903 \\
\hline $\begin{array}{l}\text { MW5-4a } \\
\text { (duplicate) }\end{array}$ & -- & -- & 44.4 & -- & 0 & -- \\
\hline mean & 3.05 & 0.3018 & 45.8 & 0.5868 & 0 & 0.6885 \\
\hline$Q C$ & -- & -- & 11.7 & -- & 12.092 & -- \\
\hline MW6-1a & 0.62 & 0.3005 & 45.2 & 1.6209 & 0 & 1.6209 \\
\hline MW6-3a & 3.94 & 0.3088 & 44.5 & 0.3275 & 0 & 0.3275 \\
\hline $502-630$ & -- & 0.2498 & -- & 0.5357 & -- & -- \\
\hline 502-914 & -- & 0.2998 & -- & 0.9986 & -- & -- \\
\hline $502-030$ & -- & 0.1013 & -- & 5.1488 & -- & -- \\
\hline SARM 41 & -- & 0.3069 & -- & 6.4372 & -- & -- \\
\hline MW6-5b & 6.80 & 0.3048 & 45.2 & 0.7471 & 0 & 0.7471 \\
\hline $\begin{array}{l}\text { MW6-5b } \\
\text { (duplicate) }\end{array}$ & -- & 0.3061 & -- & 0.7407 & - & - \\
\hline MW6-5c & 7.20 & 0.3010 & 45.7 & 1.1360 & 0 & 1.1360 \\
\hline mean & 4.64 & 0.3042 & 45.2 & 0.9144 & 0 & 0.9579 \\
\hline MW7-1a & 0.50 & 0.3042 & 44.7 & 5.8558 & 0 & 5.8558 \\
\hline$Q C$ & -- & -- & 11.7 & -- & 10.823 & -- \\
\hline MW7-2a & 2.15 & 0.3006 & 44.3 & 0.6821 & 0 & 0.6821 \\
\hline $\begin{array}{l}\text { MW7-2a } \\
\text { (duplicate) }\end{array}$ & -- & -- & 45.7 & -- & 0 & - \\
\hline MW7-4a & 5.62 & 0.3066 & 45.3 & 0.3633 & 0 & 0.3633 \\
\hline MW7-5a & 7.26 & 0.3016 & 44.8 & 0.2782 & 0 & 0.2782 \\
\hline mean & 3.88 & 0.3033 & 45.0 & 1.7949 & 0 & 1.7949 \\
\hline MW8-1a & 0.88 & 0.2998 & 46.3 & 2.4194 & 0 & 2.4194 \\
\hline QC & -- & -- & 11.6 & -- & 11.914 & -- \\
\hline
\end{tabular}


Table 14. HLDTC Soil Carbon Analytical Results (cont.)

\begin{tabular}{lrrrrrr}
\hline MW8-2a & 2.51 & 0.2976 & 46.3 & 0.4095 & 0 & 0.4095 \\
MW8-2b & 2.64 & 0.3086 & 45.6 & -0.0056 & 0 & -0.0056 \\
MW8-2c & 2.80 & 0.2997 & 45.0 & 0.1124 & 0 & 0.1124 \\
mean & 2.21 & 0.3014 & 45.8 & 0.7339 & 0 & 0.7339 \\
MW9R-1a & 0.30 & 0.3044 & 44.8 & 0.7752 & 0 & 0.7752 \\
MW9R-1c & 1.26 & 0.2951 & 43.7 & 0.1078 & 0 & 0.1078 \\
MW9R-1c & -- & 0.2985 & - & 0.1104 & -- & -- \\
(duplicate) & -- & -- & 11.5 & -- & 11.820 & -- \\
QC & --74 & 0.3006 & 45.7 & 0.1772 & 0 & 0.1772 \\
MW9R-2b & 3.63 & 0.3036 & 47.6 & 0.0345 & 0 & 0.0345 \\
MW9R-3a & 5.33 & 0.3092 & 43.4 & 0.0380 & 0 & 0.0380 \\
MW9R-4a & 2.65 & 0.3019 & 45.0 & 0.2072 & 0 & 0.2265 \\
mean & 0.32 & 0.3059 & 44.9 & 2.4088 & 0 & 2.4088 \\
MW10R-1a & 2.58 & 0.2951 & 44.3 & 0.2795 & 0 & 0.2795 \\
MW10R-2a & 5.71 & 0.3058 & 45.7 & 0.0951 & 0 & 0.0951 \\
MW10R-4a & 2.87 & 0.3023 & 45.0 & 0.9278 & 0 & 0.9278 \\
mean & 0.15 & 0.3033 & 44.2 & 5.5492 & 0 & 5.5492 \\
MW11-1a & 2.40 & 0.3022 & 44.6 & 0.2300 & 0 & 0.2300 \\
MW11-2a & 7.25 & 0.2998 & 43.9 & 0.3442 & 0 & 0.3442 \\
MW11-5a & 3.27 & 0.3018 & 44.2 & 2.0411 & 0 & 2.0411 \\
mean & -- & -- & 11.6 & -- & 11.845 & -- \\
QC & -- & 0.1491 & -- & 0.5211 & -- & -- \\
502-630 & -- & 0.0993 & -- & 0.9757 & -- & -- \\
$502-914$ & -- & 0.3094 & -- & 4.6204 & -- & -- \\
$502-030$ & -- & 0.2526 & -- & 6.4517 & -- & -- \\
SARM 41 & -- & -- & -- & 0.6540 & 0 & 0.6540 \\
mean site & & & & & &
\end{tabular}

Notes:

$\begin{aligned}-- & \text { No data } \\ \text { TC } & \text { Total Carbon } \\ \text { TIC } & \text { Total Inorganic Carbon } \\ \text { TOC } & \text { Total Organic Carbon } \\ \text { blue } & \text { Values below total carbon, LECO (low carbon method) } \\ & \text { calibration curve } \\ \text { italicized } & \text { Reference material }\end{aligned}$

$\mathrm{X}$-ray fluorescence spectroscopy (XRF) was performed to further assess soil elemental composition and its potential contribution to groundwater chemistry. The major elemental composition is predominantly silicon (24.06-38.25 wt.\%, mean 30.57 wt.\%), followed by aluminum (1.39-9.07 wt.\%, mean 6.13 wt.\%), iron $(<0.01-7.69$ wt.\%, mean 
2.05 wt. $\%)$, potassium $(<0.01-2.61$ wt.\%, mean 1.44 wt.\%), and magnesium $(0.20-4.68$ wt. $\%$, mean 0.79 wt.\%) (Table 15). Values less than zero are an analytical artifact and are considered as $<$ MDL.

Bhatia (1983) conducted a geochemical study of sands and sandstones based upon their tectonic provenance. The variations in major-element geochemistry reflect distinct sedimentary provenance and tectonic settings. The discriminating geochemical parameters are $\mathrm{Fe}_{2} \mathrm{O}_{3}+\mathrm{MgO}, \mathrm{TiO}_{2}, \mathrm{Al}_{2} \mathrm{O}_{3}, \mathrm{~K}_{2} \mathrm{O} / \mathrm{Na}_{2} \mathrm{O}$, and $\mathrm{Al}_{2} \mathrm{O}_{3} /\left(\mathrm{CaO}+\mathrm{Na}_{2} \mathrm{O}\right)$. Passive margin sands and sandstones are enriched in $\mathrm{SiO}_{2}$ and depleted in $\mathrm{Na}_{2} \mathrm{O}, \mathrm{CaO}$, and $\mathrm{TiO}_{2}$, with a $\mathrm{K}_{2} \mathrm{O} / \mathrm{Na}_{2} \mathrm{O}$ ratio of $>1$. Ranges and mean values of HLDTC soil XRF analysis for major elements (in wt.\%) align well with Bhatia's passive margin values for $\mathrm{SiO}_{2}($ Bhatia $\bar{X} 81.59$ vs. HLDTC range $51.47-81.83$ and $\bar{X} 65.39$ ), $\mathrm{TiO}_{2}$ (Bhatia $\bar{X} 0.49$ vs. HLDTC range 0.140.98 and $\bar{X} 0.675$ ), and $\mathrm{K}_{2} \mathrm{O} / \mathrm{Na}_{2} \mathrm{O}$ (Bhatia $>1$ vs. HLDTC: $\bar{X} 1.80$ ), but are less than Bhatia's value for $\mathrm{CaO}$ (Bhatia $\bar{X} 1.89$ vs. HLDTC range $<0.01-0.45$ and $\bar{X} 0.0129$ ). HLDTC XRF values are also in agreement with results of other studies (Bauluz et al., 2000; Herron, 1988; Cullers et al, 1988; Schmidt, 1973) 
Table 15. HLDTC Soil XRF Analytical Results for Major Elements (wt.\%)

\begin{tabular}{|c|c|c|c|c|c|c|c|c|c|c|}
\hline Sample ID & $\begin{array}{l}\text { Depth } \\
\text { bls } \\
(\mathrm{m})\end{array}$ & $\mathrm{Mg}$ & $\mathrm{Al}$ & $\mathrm{Si}$ & $\mathrm{K}$ & $\mathrm{Ca}$ & $\mathrm{Ti}$ & $\mathrm{Mn}$ & $\mathrm{Fe}$ & $\begin{array}{l}\text { Si:Al } \\
\text { Ratio }\end{array}$ \\
\hline MW1-1a-ma & 0.5 & 0.2981 & 3.8171 & 35.1671 & 0.6474 & 0.0545 & 0.5849 & 0.0545 & 0.9665 & 9.2130 \\
\hline MW1-2a-ma & 2 & 0.2979 & 4.8545 & 34.1973 & 0.7213 & -0.0286 & 0.5174 & 0.0299 & 1.2623 & 7.0445 \\
\hline MW1-2b-ma & 2.51 & 0.2711 & 7.8189 & 32.7295 & 1.5182 & -0.0494 & 0.5439 & 0.0328 & 0.1214 & 4.1860 \\
\hline MW1-3a-ma & 4.04 & 0.2743 & 4.6916 & 33.5787 & 0.7339 & -0.0193 & 0.5036 & 0.0321 & 0.9526 & 7.1572 \\
\hline MW1-3b-ma & 4.32 & 0.2893 & 5.1750 & 34.5379 & 0.9718 & -0.0460 & 0.2809 & 0.0351 & -0.0185 & 6.6740 \\
\hline mean & -- & 0.2861 & 5.2714 & 34.0421 & 0.9185 & -0.0178 & 0.4861 & 0.0369 & 0.6569 & 6.8549 \\
\hline MW2-1a-ma & 0.05 & 0.1984 & 6.7306 & 24.0564 & 1.7702 & 0.3151 & 0.4327 & 0.0570 & 2.3854 & 3.5742 \\
\hline MW2-1b-ma & 0.78 & 0.2666 & 7.8975 & 29.6710 & 2.0778 & 0.0025 & 0.5852 & 0.0875 & 2.3460 & 3.7570 \\
\hline MW2-2a-ma & 2.38 & 0.2607 & 8.4899 & 28.4540 & 2.1804 & -0.0350 & 0.4705 & 0.0342 & 2.7549 & 3.3515 \\
\hline MW2-5a-ma & 6.92 & 0.2999 & 4.5329 & 34.2112 & 1.2924 & -0.0283 & 0.3151 & 0.0349 & 1.3552 & 7.5473 \\
\hline mean & -- & 0.2564 & 6.9127 & & & 0.0636 & & 0.0534 & 2.2104 & 4.5575 \\
\hline MW3R-1a-ma & 0.52 & 0.3008 & 4.6585 & 34.5137 & 1.0353 & 0.0693 & 0.5052 & 0.0391 & 0.8945 & 7.4088 \\
\hline MW3R-1b-ma & 0.91 & 0.2892 & 4.7541 & 34.4281 & 1.0508 & -0.0067 & 0.5552 & 0.0315 & 0.8473 & 7.2418 \\
\hline MW3R-2b-ma & 2.77 & 0.2581 & 5.8538 & 30.7912 & 1.2854 & -0.0327 & 0.2989 & 0.0266 & 3.0825 & 5.2601 \\
\hline MW3R-3a-ma & 3.6 & 0.2502 & 5.4484 & 31.2856 & 1.2782 & -0.0360 & 0.3038 & 0.0274 & 2.7906 & 5.7421 \\
\hline MW3R-4a-ma & 5.58 & 0.2806 & 5.4521 & 33.7291 & 1.4061 & -0.0416 & 0.3012 & 0.0347 & 0.2488 & 6.1865 \\
\hline mean & -- & 0.2758 & 5.2334 & 32.9495 & 1.2112 & -0.0095 & 0.3929 & 0.0319 & 1.5727 & 6.3679 \\
\hline MW4-1a-ma & 0.57 & 0.2776 & 7.9957 & 29.5306 & 2.0466 & -0.0101 & 0.5679 & 0.0912 & 2.3461 & 3.6933 \\
\hline MW4-2a-ma & 2.45 & 0.2751 & 7.9206 & 29.6556 & 1.9920 & -0.0508 & 0.3971 & 0.0351 & 2.5774 & 3.7441 \\
\hline MW4-5b-ma & 7.05 & 0.2801 & 5.6090 & 33.2757 & 1.4382 & -0.0130 & 0.3064 & 0.0377 & 1.0897 & 5.9325 \\
\hline mean & -- & 0.2776 & 7.1751 & 30.8206 & 1.8256 & -0.0246 & 0.4238 & 0.0547 & 2.0044 & 4.4566 \\
\hline MW5-1a-ma & 0.51 & 0.2526 & 6.5339 & 30.0077 & 1.7445 & 0.1459 & 0.4034 & 0.0636 & 2.2068 & 4.5926 \\
\hline MW5-2a-ma & 1.94 & 0.2546 & 6.5717 & 30.5396 & 1.7517 & -0.0205 & 0.4133 & 0.0571 & 2.2161 & 4.6471 \\
\hline MW5-3b-ma & 3.99 & 0.2628 & 6.7069 & 30.7475 & 1.7434 & -0.0262 & 0.3845 & 0.0524 & 2.4902 & 4.5845 \\
\hline MW5-4a-ma & 5.75 & 0.2773 & 4.0952 & 33.7950 & 1.1688 & -0.0191 & 0.2475 & 0.0520 & 1.7891 & 8.2524 \\
\hline mean & -- & 0.2618 & 5.9769 & 31.2725 & 1.6021 & 0.0200 & 0.3622 & 0.0563 & 2.1756 & 5.5192 \\
\hline
\end{tabular}


Table 15. HLDTC Soil XRF Analytical Results for Major Elements (wt.\%) (cont.)

\begin{tabular}{|c|c|c|c|c|c|c|c|c|c|c|}
\hline MW6-1a-ma & 0.62 & 0.2736 & 9.0730 & 27.9632 & 2.6063 & 0.0388 & 0.5290 & 0.0448 & 2.2412 & 3.0820 \\
\hline MW6-3a-ma & 3.94 & 0.3360 & 7.6338 & 29.0714 & 2.1492 & 0.0044 & 0.4268 & 0.1186 & 2.6018 & 3.8083 \\
\hline MW6-5b-ma & 6.8 & 0.3184 & 6.5899 & 31.4950 & 1.9824 & -0.0081 & 0.4183 & 0.0331 & 1.4194 & 4.7792 \\
\hline MW6-5c-ma & 7.2 & 0.3240 & 6.1745 & 31.6450 & 1.8004 & -0.0014 & 0.3874 & 0.0319 & 1.9504 & 5.1251 \\
\hline mean & -- & 0.3130 & 7.3678 & 30.0437 & 2.1346 & 0.0084 & 0.4403 & 0.0571 & 2.0532 & 4.1987 \\
\hline MW7-1a-ma & 0.5 & 0.2231 & 7.2435 & 27.2610 & 2.0797 & 0.1305 & 0.4536 & 0.0666 & 2.3305 & 3.7635 \\
\hline MW7-2a-ma & 2.15 & 0.2570 & 6.9724 & 29.7512 & 1.8812 & 0.0993 & 0.3941 & 0.0635 & 2.1720 & 4.2670 \\
\hline MW7-4a-ma & 5.62 & 0.2722 & 7.0335 & 29.9328 & 1.8867 & 0.0094 & 0.4279 & 0.0661 & 2.4153 & 4.2558 \\
\hline MW7-5a-ma & 7.26 & 0.2639 & 4.9993 & 32.2704 & 1.3995 & -0.0106 & 0.3016 & 0.0538 & 1.5858 & 6.4549 \\
\hline mean & -- & 0.2541 & 6.5622 & 29.8039 & 1.8118 & 0.0572 & 0.3943 & 0.0625 & 2.1259 & 4.6853 \\
\hline MW8-1a-ma & 0.88 & 0.2920 & 3.6229 & 34.9859 & 0.6365 & 0.0146 & 0.4884 & 0.0446 & 0.6220 & 9.6570 \\
\hline MW8-2a-ma & 2.51 & 0.2970 & 5.9731 & 34.3390 & 1.0304 & -0.0327 & 0.3921 & 0.0345 & 0.4480 & 5.7489 \\
\hline MW8-2b-ma & 2.64 & 0.3495 & 1.3902 & 38.2647 & -0.0923 & -0.0307 & 0.0868 & 0.0403 & -0.4960 & 27.524 \\
\hline MW8-2c-ma & 2.8 & 0.2300 & 4.7678 & 29.3313 & 0.8438 & -0.0392 & 0.2287 & 0.0251 & 5.8524 & 6.1519 \\
\hline mean & -- & 0.2921 & 3.9385 & 34.2302 & 0.6046 & -0.022 & 0.299 & 0.0361 & 1.6066 & 12.271 \\
\hline MW9R-1a-ma & 0.3 & 0.2640 & 5.6348 & 32.4885 & 0.8966 & 0.0333 & 0.5553 & 0.1765 & 0.8849 & 5.7657 \\
\hline MW9R-1c-ma & 1.26 & 0.2398 & 7.3587 & 28.7122 & 1.2479 & -0.0207 & 0.3655 & 0.0210 & 3.8323 & 3.9018 \\
\hline MW9R-2b-ma & 2.74 & 0.1977 & 3.3447 & 25.4896 & 0.4954 & -0.0303 & 0.2037 & 0.0058 & 7.6938 & 7.6209 \\
\hline MW9R-3a-ma & 3.63 & 0.2896 & 4.1012 & 34.6530 & 0.9102 & -0.0352 & 0.2623 & 0.0401 & 0.7224 & 8.4494 \\
\hline MW9R-4a-ma & 5.33 & 0.3027 & 3.6142 & 35.7232 & 0.8274 & -0.0417 & 0.2412 & 0.0346 & 0.5044 & 9.8842 \\
\hline mean & -- & 0.2588 & 4.8107 & 31.4133 & 0.8755 & -0.0189 & 0.3256 & 0.0556 & 2.7276 & 7.1244 \\
\hline MW10R-1a-ma & 0.32 & 0.3014 & 7.7911 & 28.7918 & 2.2154 & 0.1168 & 0.5115 & 0.0778 & 2.2786 & 3.6955 \\
\hline MW10R-2a-ma & 2.58 & 0.3493 & 7.8128 & 29.5476 & 2.0603 & -0.0396 & 0.4426 & 0.0631 & 2.6348 & 3.7819 \\
\hline MW10R-4a-ma & 5.71 & 0.3161 & 8.2490 & 30.2562 & 2.1842 & -0.0316 & 0.4577 & 0.0323 & 1.4871 & 3.6679 \\
\hline mean & -- & 0.3223 & 7.9510 & 29.5319 & 2.1533 & 0.0152 & 0.4706 & 0.0577 & 2.1335 & 3.7151 \\
\hline MW11-1a-ma & 0.15 & 0.2654 & 7.4245 & 28.0100 & 2.0934 & 0.1514 & 0.4600 & 0.0647 & 2.2744 & 3.7726 \\
\hline MW11-2a-ma & 2.4 & 0.2705 & 8.6460 & 28.0339 & 2.3026 & -0.0138 & 0.4699 & 0.0763 & 2.7364 & 3.2424 \\
\hline MW11-5a-ma & 7.25 & 0.2866 & 7.5075 & 28.2913 & 1.9423 & 0.0220 & 0.3934 & 0.0621 & 2.8704 & 3.7684 \\
\hline mean & -- & 0.2742 & 7.8593 & 28.1117 & 2.1128 & 0.0532 & 0.4411 & 0.0677 & 2.6271 & 3.5944 \\
\hline mean site & 3.04 & 0.2781 & 6.1038 & 31.2548 & 1.4826 & 0.0093 & 0.4049 & 0.0505 & 1.9493 & 5.9536 \\
\hline
\end{tabular}


Table 15. HLDTC Soil XRF Analytical Results for Major Elements (wt.\%) (cont.)

\begin{tabular}{lllllllllll}
\hline SARM41-end-ma & -- & 4.4216 & 6.2795 & 26.1128 & 1.1333 & 1.0708 & 0.2885 & 0.0484 & 2.6718 & -- \\
SARM41-start-ma & -- & 4.6814 & 6.4815 & 26.2112 & 1.1422 & 1.0588 & 0.2606 & 0.0491 & 2.5215 & -- \\
SARM41-end-ma & -- & 3.0447 & 5.9303 & 26.8675 & 1.1466 & 1.0105 & 0.2758 & 0.0500 & 2.6917 & -- \\
SARM41-start-ma & -- & 4.2887 & 6.3566 & 25.9972 & 1.1232 & 1.0506 & 0.2896 & 0.0488 & 2.4579 & -- \\
SARM41-end-ma & -- & 3.9761 & 6.3964 & 25.8557 & 1.1412 & 1.0456 & 0.2901 & 0.0491 & 2.9484 & -- \\
SARM41-mid-ma & -- & 4.0177 & 6.2770 & 26.7279 & 1.1442 & 1.0479 & 0.2983 & 0.0495 & 2.7329 & -- \\
SARM41-start-ma & -- & 3.8654 & 6.4204 & 26.0463 & 1.1532 & 1.0725 & 0.2881 & 0.0479 & 2.5614 & -- \\
\hline Notes: & & & & & & & & & &
\end{tabular}


Table 16. HLDTC Soil XRF Analytical Results for Minor Elements (wt.\%)

\begin{tabular}{|c|c|c|c|c|c|c|}
\hline Sample ID & $\mathrm{Cr}$ & $\mathrm{Mn}$ & $\mathrm{Fe}$ & $\mathrm{Cu}$ & $\mathrm{Zn}$ & Mo \\
\hline MW1-1a-tr & 0.0018 & 0.0355 & 1.8290 & 0.0011 & 0.0052 & 0.0002 \\
\hline MW1-2a-tr & 0.0086 & 0.0131 & 2.0710 & 0.0006 & 0.0017 & 0.0000 \\
\hline MW1-2b-tr & -0.0024 & 0.0152 & 0.8304 & 0.0006 & 0.0017 & 0.0007 \\
\hline MW1-3a-tr & 0.0540 & 0.0718 & 1.2009 & 0.0185 & 0.0120 & 0.0085 \\
\hline MW1-3b-tr & 0.0637 & 0.0690 & 0.7813 & 0.0166 & 0.0118 & 0.0085 \\
\hline mean & 0.0251 & 0.0409 & 1.3425 & 0.0075 & 0.0065 & 0.0036 \\
\hline MW2-1a-tr & -0.0008 & 0.0657 & 3.3180 & 0.0021 & 0.0094 & 0.0011 \\
\hline MW2-1b-tr & -0.0030 & 0.0770 & 3.3997 & 0.0021 & 0.0084 & 0.0003 \\
\hline MW2-2a-tr & 0.0041 & 0.0296 & 4.2201 & 0.0020 & 0.0077 & 0.0009 \\
\hline MW2-5a-tr & 0.0661 & 0.0786 & 1.5458 & 0.0184 & 0.0121 & 0.0085 \\
\hline mean & 0.0166 & 0.0627 & 3.1209 & 0.0062 & 0.0094 & 0.0027 \\
\hline MW3R-1a-tr & -0.0003 & 0.0229 & 1.6210 & 0.0007 & 0.0034 & 0.0005 \\
\hline MW3R-1b-tr & 0.0028 & 0.0162 & 1.7619 & 0.0011 & 0.0030 & 0.0001 \\
\hline MW3R-2b-tr & 0.0709 & 0.0831 & 2.7058 & 0.0192 & 0.0114 & 0.0085 \\
\hline MW3R-3a-tr & 0.0683 & 0.0779 & 2.5159 & 0.0195 & 0.0117 & 0.0085 \\
\hline MW3R-4a-tr & 0.0608 & 0.0703 & 0.8705 & 0.0177 & 0.0120 & 0.0085 \\
\hline mean & 0.0405 & 0.0541 & 1.8950 & 0.0116 & 0.0083 & 0.0052 \\
\hline MW4-1a-tr & -0.0047 & 0.0777 & 3.2391 & 0.0016 & 0.0090 & 0.0006 \\
\hline MW4-2a-tr & 0.0615 & 0.0929 & 1.9739 & 0.0200 & 0.0125 & 0.0085 \\
\hline MW4-5b-tr & 0.0628 & 0.0805 & 1.4132 & 0.0185 & 0.0121 & 0.0085 \\
\hline mean & 0.0399 & 0.0837 & 2.2087 & 0.0134 & 0.0112 & 0.0059 \\
\hline MW5-1a-tr & 0.0040 & 0.0522 & 2.7391 & 0.0014 & 0.0065 & 0.0009 \\
\hline MW5-2a-tr & 0.0046 & 0.0448 & 2.7596 & 0.0015 & 0.0052 & 0.0006 \\
\hline MW5-3b-tr & 0.0054 & 0.0420 & 3.1059 & 0.0016 & 0.0059 & 0.0007 \\
\hline MW5-4a-tr & 0.0822 & 0.0990 & 1.8641 & 0.0187 & 0.0120 & 0.0085 \\
\hline mean & 0.0241 & 0.0595 & 2.6172 & 0.0058 & 0.0074 & 0.0027 \\
\hline MW6-1a-tr & -0.0014 & 0.0422 & 3.4600 & 0.0026 & 0.0107 & 0.0009 \\
\hline MW6-3a-tr & 0.0508 & 0.1890 & 1.5921 & 0.0205 & 0.0133 & 0.0085 \\
\hline MW6-5b-tr & 0.0565 & 0.0783 & 1.4466 & 0.0187 & 0.0127 & 0.0085 \\
\hline MW6-5c-tr & 0.0649 & 0.0823 & 1.8673 & 0.0191 & 0.0126 & 0.0085 \\
\hline mean & 0.0427 & 0.0980 & 2.0915 & 0.0152 & 0.0123 & 0.0066 \\
\hline MW7-1a-tr & 0.0011 & 0.0579 & 3.2819 & 0.0028 & 0.0101 & 0.0012 \\
\hline MW7-2a-tr & 0.0045 & 0.0533 & 3.0590 & 0.0018 & 0.0070 & 0.0009 \\
\hline MW7-4a-tr & 0.0048 & 0.0527 & 3.1234 & 0.0014 & 0.0066 & 0.0005 \\
\hline MW7-5a-tr & 0.0653 & 0.1027 & 1.7244 & 0.0187 & 0.0121 & 0.0085 \\
\hline mean & 0.0189 & 0.0667 & 2.7972 & 0.0062 & 0.0090 & 0.0028 \\
\hline MW8-1a-tr & 0.0026 & 0.0261 & 1.4323 & 0.0014 & 0.0062 & 0.0009 \\
\hline MW8-2a-tr & 0.0650 & 0.0699 & 0.9966 & 0.0173 & 0.0124 & 0.0085 \\
\hline MW8-2b-tr & 0.0117 & 0.0138 & 0.4231 & -0.0008 & -0.0007 & 0.0041 \\
\hline MW8-2c-tr & 0.0324 & 0.0207 & 6.6973 & 0.0015 & 0.0018 & 0.0017 \\
\hline mean & 0.0279 & 0.0326 & 2.3873 & 0.0049 & 0.0049 & 0.0038 \\
\hline
\end{tabular}


Table 16. HLDTC Soil XRF Analytical Results for Minor Elements (wt.\%) (cont.)

\begin{tabular}{lrrrrrr}
\hline MW9R-1a-tr & -0.0073 & 0.1068 & 1.7028 & 0.0007 & 0.0041 & 0.0005 \\
MW9R-1c-tr & 0.0126 & 0.0154 & 4.7357 & 0.0015 & 0.0045 & 0.0008 \\
MW9R-2b-tr & 0.0277 & 0.0024 & 9.9136 & 0.0011 & 0.0006 & 0.0012 \\
MW9R-3a-tr & 0.0598 & 0.0777 & 1.1581 & 0.0178 & 0.0121 & 0.0085 \\
MW9R-4a-tr & 0.0667 & 0.0708 & 1.0892 & 0.0170 & 0.0117 & 0.0085 \\
mean & 0.0319 & 0.0546 & 3.7199 & 0.0076 & 0.0066 & 0.0039 \\
MW10R-1a-tr & -0.0021 & 0.0675 & 3.3314 & 0.0025 & 0.0095 & 0.0004 \\
MW10R-2a-tr & 0.0017 & 0.0537 & 3.6620 & 0.0021 & 0.0083 & 0.0011 \\
MW10R-4a-tr & 0.0447 & 0.0803 & 1.2675 & 0.0192 & 0.0131 & 0.0085 \\
mean & 0.0148 & 0.0672 & 2.7536 & 0.0079 & 0.0103 & 0.0033 \\
MW11-1a-tr & 0.0003 & 0.0554 & 3.1818 & 0.0026 & 0.0090 & 0.0012 \\
MW11-2a-tr & -0.0027 & 0.0672 & 3.7358 & 0.0021 & 0.0089 & 0.0001 \\
MW11-5a-tr & 0.0619 & 0.1320 & 2.1149 & 0.0207 & 0.0129 & 0.0085 \\
mean & 0.0198 & 0.0848 & 3.0108 & 0.0085 & 0.0103 & 0.0033 \\
mean site & 0.0280 & 0.0621 & 2.5173 & 0.0085 & 0.0085 & 0.0040 \\
SARM41-end-tr & 0.0689 & 0.1090 & 2.3828 & 0.0201 & 0.0119 & 0.0085 \\
SARM41-start-tr & 0.0709 & 0.1097 & 2.5186 & 0.0202 & 0.0128 & 0.0085 \\
SAMR41-start-tr & 0.0084 & 0.0424 & 3.5346 & 0.0045 & 0.0070 & 0.0029 \\
SARM41-mid-tr & 0.0079 & 0.0422 & 3.4316 & 0.0043 & 0.0076 & 0.0026 \\
SARM41-end-tr & 0.0079 & 0.0420 & 3.4387 & 0.0046 & 0.0078 & 0.0027 \\
\hline
\end{tabular}

The Si:Al ratio was calculated (Table 15) and graphed vs. depth (Figure 17). Higher $\mathrm{Si}$ Al values are indicative of higher rates of weathering based upon Bowen's reaction series. The Si:Al ratio ranged from 3.08 to $27.5 \mathrm{wt} . \%$ (mean 5.95\%). Figure 16 depicts that weathering rates are fairly consistent from 0.05 to $7.26 \mathrm{~m}$ bls, with values falling between $3.08-9.88$ wt. $\%$, except for the outlier value of $27.5 \%$ at a depth of $2.64 \mathrm{~m}$ bls in MW-8.

In humid climates, the Ruxton $\left(\mathrm{SiO}_{2} / \mathrm{Al}_{2} \mathrm{O}_{3}\right)$ ratio provides a simple way to quantify the degree of rock weathering. The ratio premise is that silica loss is correlated to total element loss, whereas alumina is conserved (Ruxton, 1968). Hence, changes in the ratio indicate the degree of weathering; a ratio $>10$ is fresh or not weathered and 0 is completely weathered (Price and Velbel, 2002). Literature shows that gradual or continuous, steady and systematic weathering with depth, as evident in Figure 16, is observed within homogeneous rocks, soils, and sediments, reflecting continuous leaching of elements as 
weathering progresses within the original material (Minasny et al., 2016; Price and Velbel, 2002; Zhang and Hartemink, 2018). The $\mathrm{SiO}_{2} / \mathrm{Al}_{2} \mathrm{O}_{3}$ mean ratio (3.37 wt.\%) indicates that the HLDTC alluvium is moderately weathered according to Ruxton ratio.

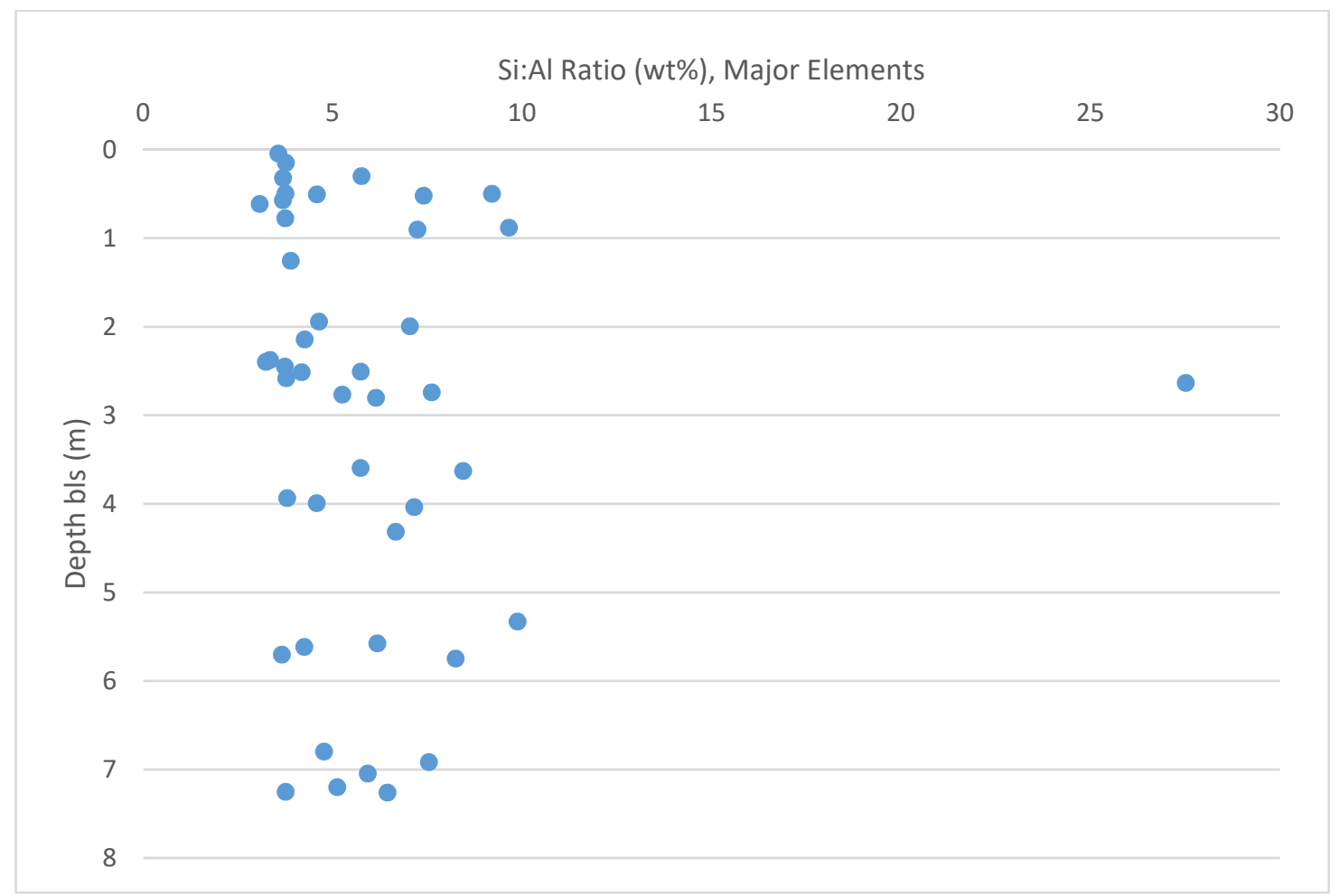

Figure 17: HLDTC Al:Si ratios in soil cores as a function of depth.

Major and minor (trace) analyses showed fairly consistent ranges for both manganese ( $\mathrm{Mn})$ and iron (Fe) with depth (Figure 18). Manganese concentrations ranged from $0.0058-0.177$ wt. $\%$ for major analyses (mean 0.0505 wt. \%) and $0.0024-0.189$ wt.\% for minor analyses (Table 16; mean $0.0621 \%$ ), with concentrations $<0.012 \mathrm{wt} . \%$ except at $3.94 \mathrm{~m}$ bls in MW-6, $0.3 \mathrm{~m}$ bls in MW-9R, and $7.25 \mathrm{~m}$ bls in MW-11 (minor analysis). Iron concentrations ranged from $<0.0001-7.69 \mathrm{wt} . \%$ for major analyses (mean $1.95 \mathrm{wt} . \%$ ) and $0.4231-9.91 \mathrm{wt} . \%$ for minor analyses (Table 16; mean $2.52 \mathrm{wt.} \%$ ), with concentrations $<5$ wt. $\%$ except at $2.8 \mathrm{~m}$ bls in MW-8 and $2.74 \mathrm{~m}$ bls in MW-9R. The Fe outlier in MW8 occurs just below the Si:Al outlier in the same core. 

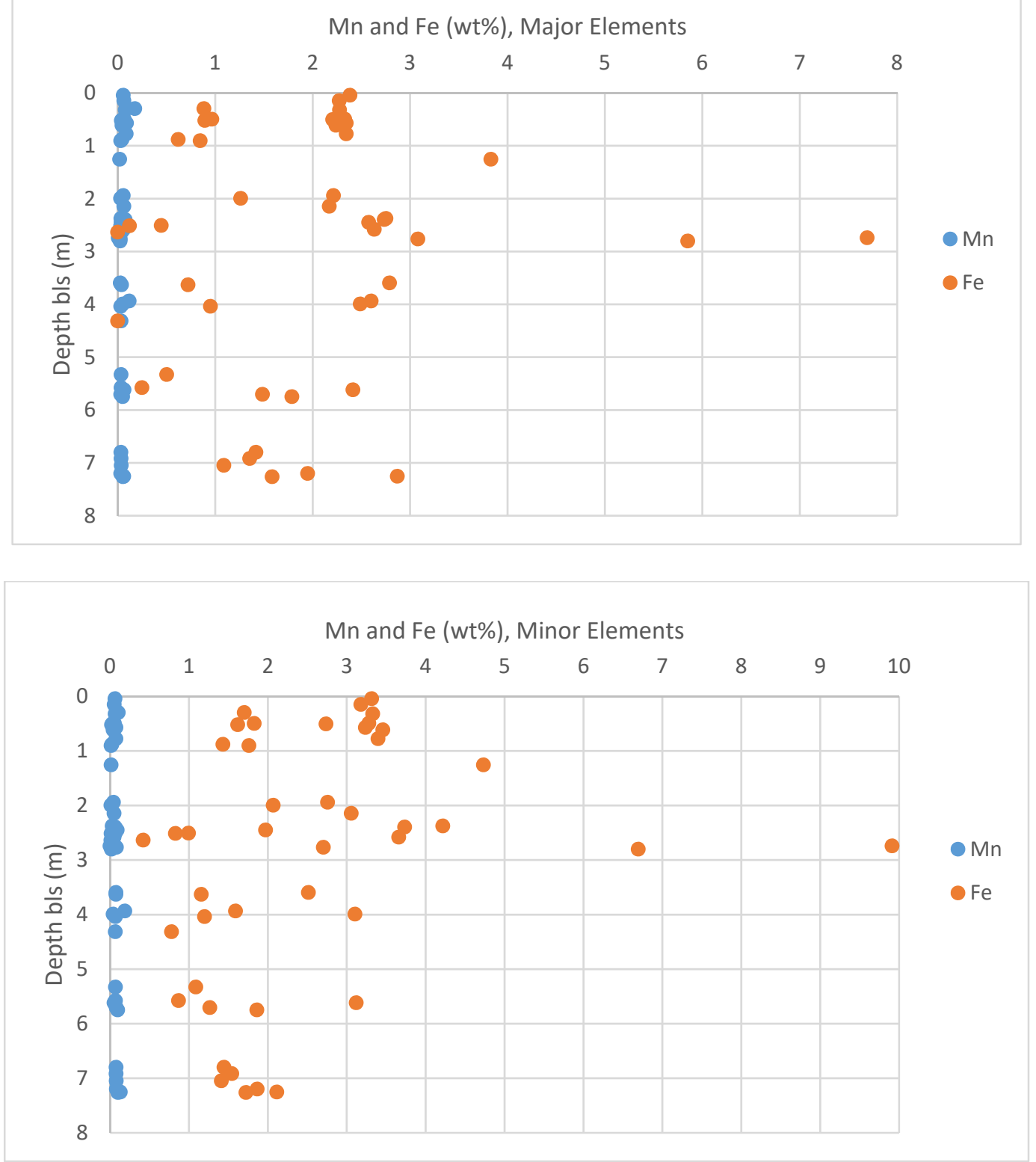

Figure 18: HLDTC Mn and Fe concentrations in soil core samples measured by major(top) and minor- (bottom) element XRF analysis as a function of depth.

Expressed as $\mathrm{MnO}$ and $\mathrm{FeO}$, the HLDTC major-element XRF results (in wt.\%) align well with Bhatia's mean values for passive margin sands and sandstones $(\mathrm{MnO}$ $\bar{X} 0.05$, HLDTC range $0.0075-0.23, \bar{X} 0.07$; FeO $\bar{X} 1.76$, HLDTC range $<0.01-9.89$, $\bar{X}$ 1.31). The abundances of $\mathrm{Fe}$ and $\mathrm{Mn}$ are a reflection of redox conditions (Price and Velbel, 2002). X-ray fluorescence analyses for trace elements exhibit wide variability in 
sand and sandstones due to effects and degrees of weathering. For example, smaller-radii ions such as $\mathrm{Na}, \mathrm{Ca}$ and $\mathrm{Sr}$ are selectively leached, while larger-radii ions such as $\mathrm{K}, \mathrm{Cs}$, $\mathrm{Rb}$, and $\mathrm{Ba}$ can be fixed by preferential exchange and adsorption to clays, and other elements such as $\mathrm{Cr}, \mathrm{Co}, \mathrm{Ni}, \mathrm{Ti}$, and V may be fractionated (Bauluz et al., 2000).

\subsection{Site Hydrogeology}

\subsubsection{Groundwater Flow}

Depth to the water table was typically shallowest in upslope wells near the ridge and increased with proximity to the river. Minimum depths to water ranged from $<1 \mathrm{~m}$ bls in MW-8 and MW-3R to $>4 \mathrm{~m}$ bls in MW-7 and MW-11, while maximum depths ranged from $<2 \mathrm{~m}$ bls in MW-8 and MW-3R to $>4.5 \mathrm{~m}$ bls in MW-7, MW-10R, MW-11, and MW-2. Water levels were highest in April 2017 with the exception of wells MW-4 and MW-6 (which were highest in January 2017) and MW-8 (which was highest in both January and April 2017). Water levels were lowest in October 2017 except for MW-10R (January 2017), MW-11 (July 2017), and MW-3R (January 2018). Depth to water was consistently greater in the tree-farm well (range $14.36 \mathrm{~m}$ bls in January 2017 to $17.10 \mathrm{~m}$ bls in October 2017), which indicates a downward hydraulic gradient between the alluvium and bedrock in the south-central portion of the site.

Quarterly water-table maps were created to evaluate site groundwater flow directions. Flow is inferred to be perpendicular to hydraulic-head contours, which were similar for each quarter (Figure 19), indicating that overall flow direction is from the toe of the ridge toward the Cumberland River, consistent with topographically driven groundwater flow (Wunsch, 1993; Fetter, 1994). Hydraulic-head contours are more closely spaced on the eastern portion of the site where the floodplain is narrow, indicating a steeper 
lateral hydraulic gradient, than on the western portion, where the floodplain is relatively flat and broad.
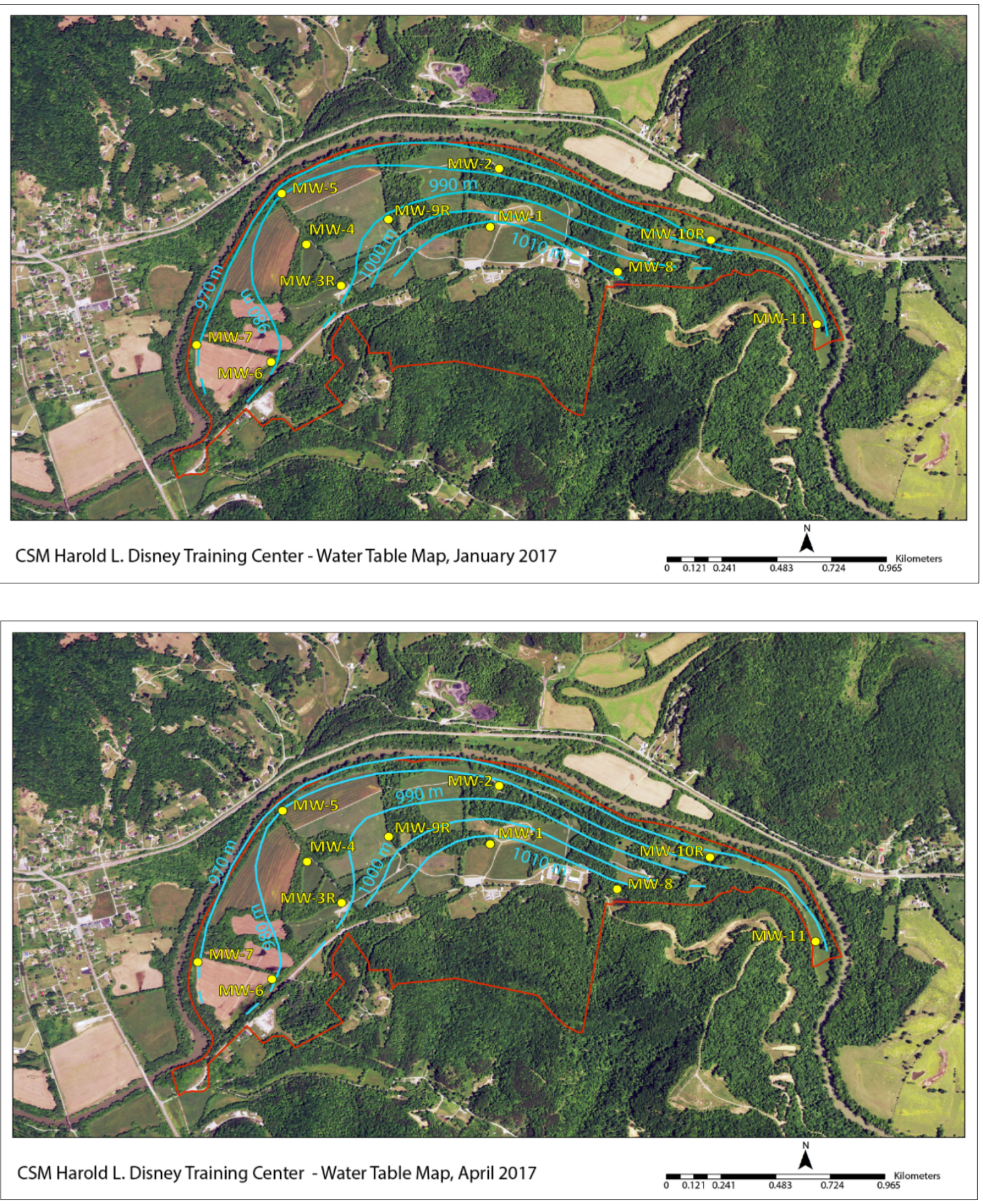

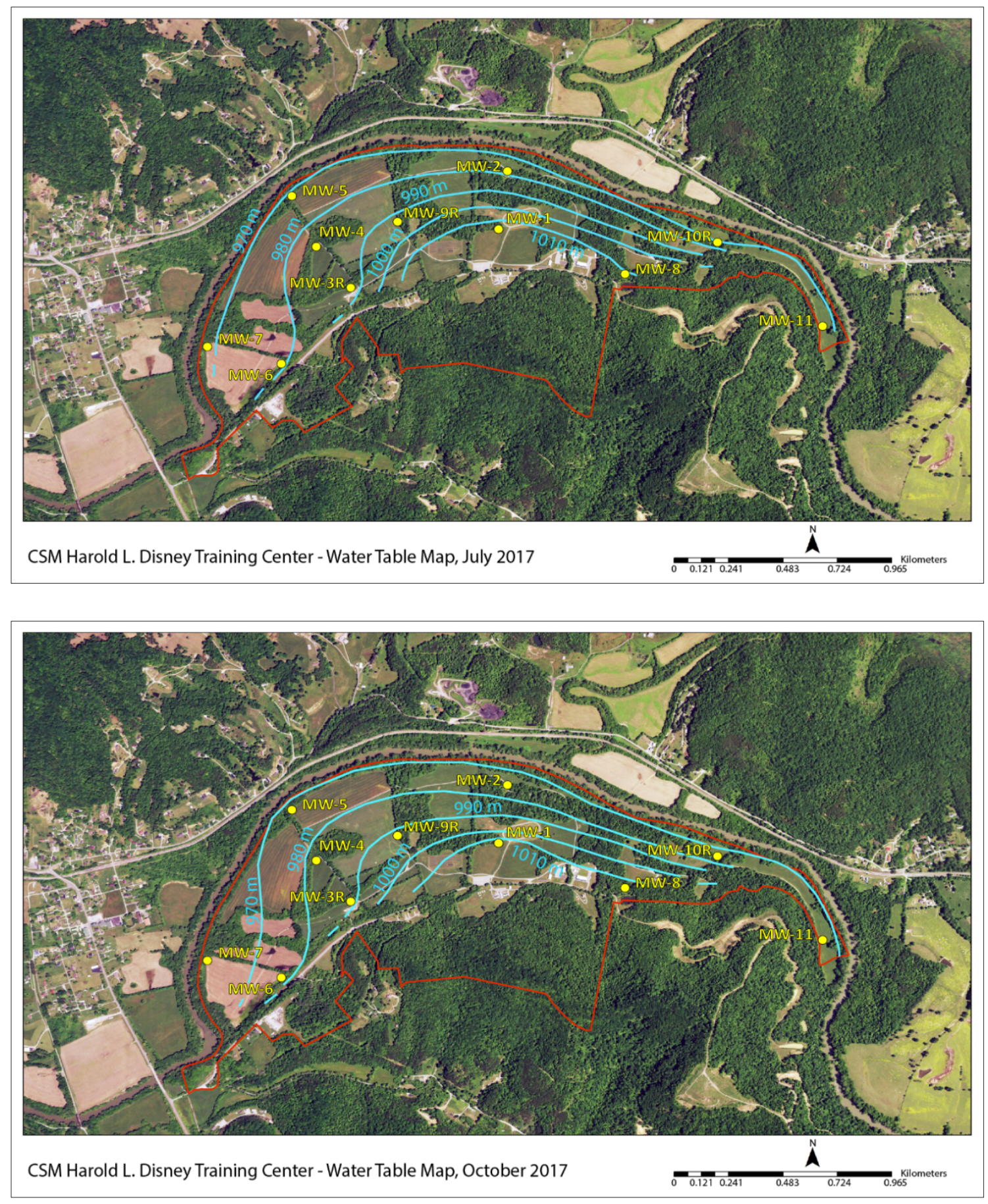

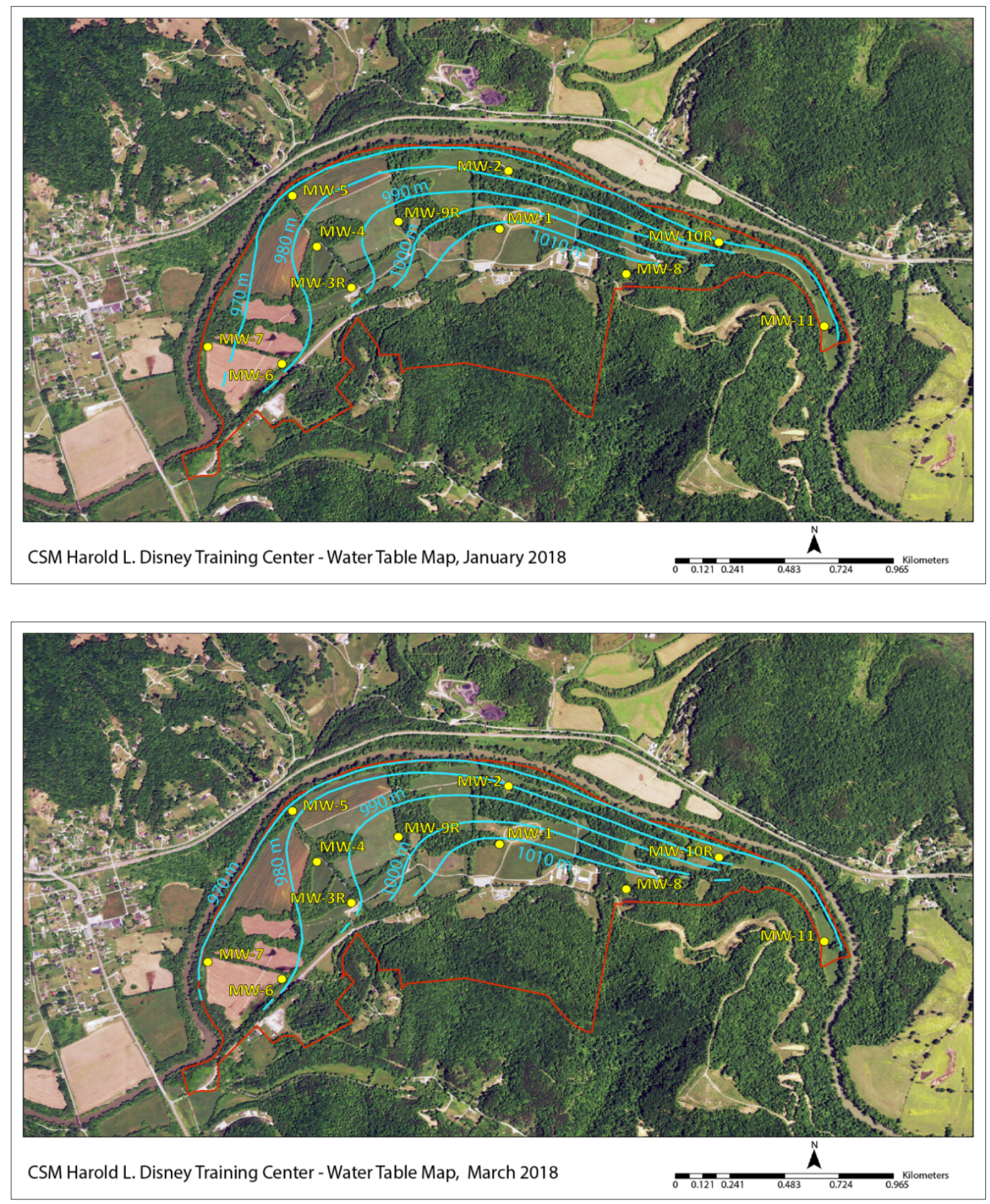

Figure 19: HLDTC water-table map from January 2017 to March 2018. Contours are in meters above sea level.

Other studies in eastern Kentucky observed similar seasonal trends for water-table elevations. Wunsch (1993) noted a maximum in February and minimum in November, while Minns (1993) observed that water levels rose in winter to early spring and declined 
in late summer to fall. Both attributed the observations to maximum recharge occurring in the fall/winter when precipitation exceeds evapotranspiration and infiltration is greatest. Wunsch (1993) further observed an even distribution of precipitation frequency and intensity in the fall/winter, whereas late spring/summer exhibited strong storm fronts with generation of runoff rather than infiltration. The water table exhibited a quick and distinct response to precipitation events in shallow wells, indicating a zone of high vertical hydraulic conductivity and direct infiltration (Wunsch, 1993). Within the Cumberland Plateau, a strong correlation exists between precipitation and recharge, which is heavily climate dependent (McCoy et al., 2015).

Considering water-table response to precipitation and the similarity of groundwater chemistry in shallow wells to precipitation chemistry, it can be surmised that the alluvial aquifer at HLDTC is recharged by precipitation. Therefore, the local groundwater flow is driven by vertical infiltration and flow from the ridge toward the river. The spacing of equipotential lines suggests a steeper hydraulic gradient in the eastern portion of the site relative to the western portion. Kipp and Dinger (1993) proposed a conceptual model to explain local, shallow groundwater flow within the eastern Kentucky coal field. Groundwater flow is described as cascading or stair-step: steep slopes are associated with runoff; where there are breaks in slope, recharge occurs; and downgradient flow discharges below local drainage. Groundwater flows vertically downward until intercepting a horizontal unit with high hydraulic conductivity, which is commonly ascribed to fractures between bedding planes or coal beds due to cleating (secondary fracturing in coals). The result is dewatering of the overlying rock units by horizontal flow that escapes along hillsides as seeps and/or springs. The escaped water can reenter the groundwater by 
infiltration and repeat the pattern until final discharge below local drainage to a surfacewater body. This conceptual model is supported by the geochemical study of Wunsch (1993) and appears to apply to groundwater flow at HLDTC.

Minns (1993) proposed a conceptual model for the eastern Kentucky coal field that considers local and regional groundwater flow. The model is based on the presence of five groundwater recharge zones. The first two, the above-Magoffin-member zone and the below-and-including Magoffin-member zone, are identified by distinctive water chemistry and are not applicable to HLDTC because the Magoffin member (an upper unit of the Breathitt Fm.) is absent there. The other three zones (shallow-fracture, elevation-head, and pressure-head) are identified by hydraulic properties. According to Minns (1993), smallscale local flow systems, such as the cascading flow described by Kipp and Dinger (1993), develop in response to topography and are contained within the shallow-fracture zone $\sim 15$ to $21 \mathrm{~m}$ bls. Beneath this zone, the elevation-head zone is located above local drainage and is $\sim 70 \%$ of the above-drainage strata. These strata contain horizontally conductive coal beds, which may laterally relieve pressure by functioning as drains, and are interbedded with sandstones and shales, which have low vertical hydraulic conductivity. Water within the elevation-head zone discharges to first- to third-order streams. Water that is not discharged flows downward to the pressure-head zone, which is below local drainage. Within this zone, groundwater is confined, strata have similar values of hydraulic conductivity, and flow occurs at the regional scale. Flow within this zone proceeds downward until intercepting the freshwater-saline interface, then moves along the interface toward third-order or larger streams, where it discharges. Although determining regional 
flow was beyond the current project scope, it is reasonable to conclude that the conceptual model of Minns (1993) for both local and regional flow would apply at HLDTC.

Hydrographs for MW-6 and MW-7 are plotted along with interpolated stage data, based on the approximate linear distance of HLDTC between the Pineville and Barbourville gages, and the average precipitation at the gages from January 2017 to March 2018 (Figure 20). Approximately seven gradient reversals occurred where groundwater moved away from the river; these corresponded with increased precipitation periods. The pressure wave from the stage rise was observed both in MW-7, which is close to the Cumberland River ( $\sim 50 \mathrm{~m})$, and MW-6, which is further from the river ( $\sim 400 \mathrm{~m})$ and close to the toe of the ridge, within $24 \mathrm{hr}$ of stage rise. These events occurred on January 23, 2017; March 2, 2017; April 3, 2017; April 21, 2017; May 27, 2017; December 22, 2017; and February 4, 2018. This last event had the longest duration ( $~ 8$ days at MW-6 and 10 days at MW-7). Prior events had a duration of 1 day in MW-6 (April 21, 2017) and a mean duration of 3.4 days in MW-7. For each event, the river stage exceeded the hydraulic head in MW-7. For the February 2018 event, the hydraulic head in MW-7 exceeded the hydraulic head in MW-6 (Figure 21). In general, hydraulic heads in the alluvial aquifer mimicked river stage, but the head rise in the monitoring wells was increasingly attenuated and dampened with distance from the Cumberland River.

Maharjan and Donovan (2016) studied groundwater responses to changes in river stage within unconfined alluvial aquifers along the Ohio River in West Virginia. In general, aquifer hydraulic heads mimicked the stream hydrographs, where river stage controls the aquifer during peak flow and vice versa during low flow. Flood waves induce lateral infiltration of river water into the alluvial aquifer through the riparian zone, which reverses 
when base flow is restored. The water table in the riparian zone does not drop as fast as the stream stage recedes. There was a $14-95-\mathrm{hr}$ lag time between peak stage and increases in aquifer hydraulic heads. The timeframe is dependent on local riparian-zone and aquifer properties. The flood pulse decreased in amplitude and increased in lag time with distances further from the river. Gradient reversals only occurred in the alluvial aquifer during peak flood events.

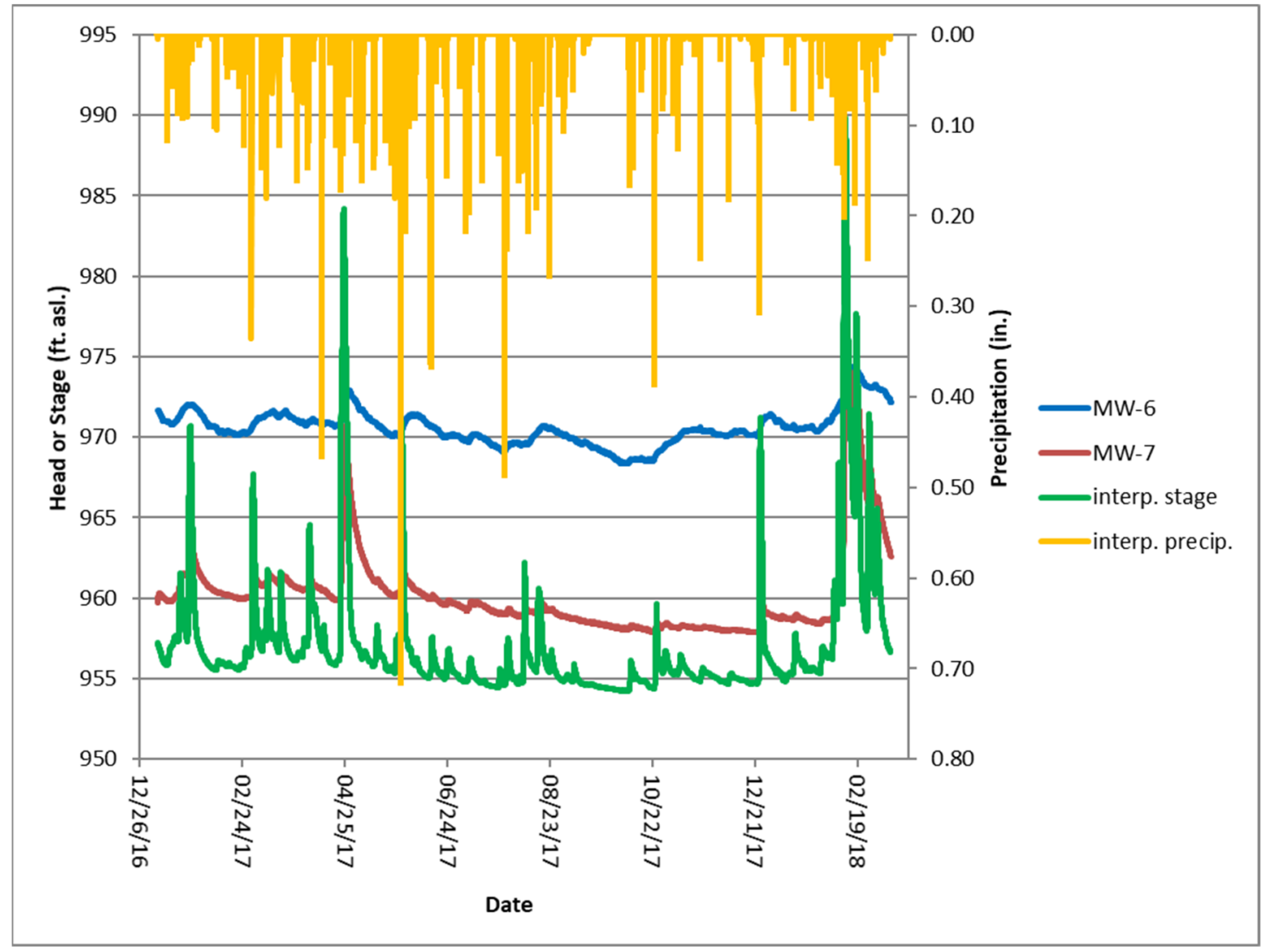

Figure 20: HLDTC hydrographs for MW-6 and MW-7 with interpolated Cumberland River stage and 24-hr precipitation totals January 2017 to March 2018. Note $1.00 \mathrm{ft}=0.305 \mathrm{~m}$ and $1.00 \mathrm{in}=2.54 \mathrm{~cm}$. 


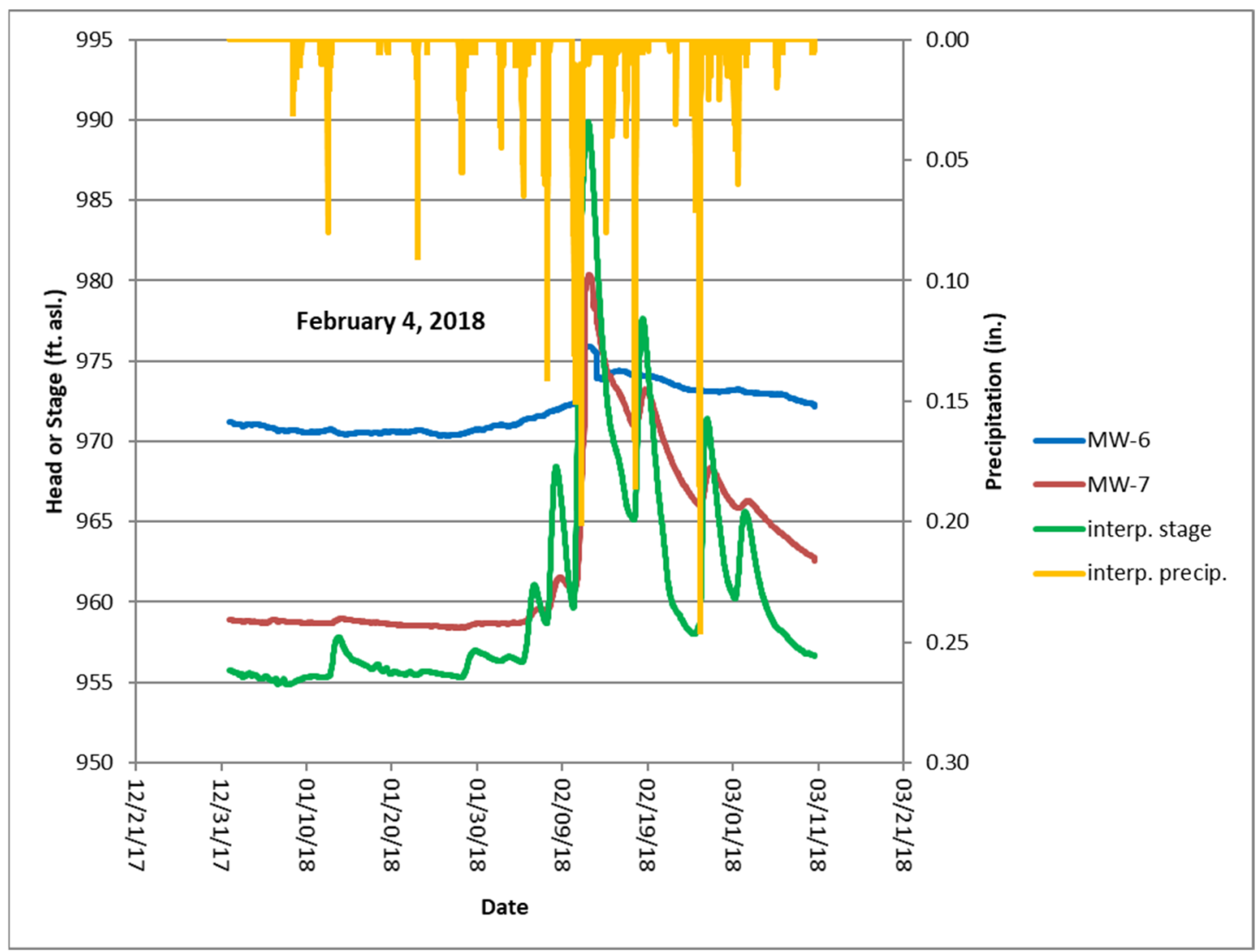

Figure 21: HLDTC hydrographs detailing gradient reversal during February 2018 in MW6 and $M W-7$ with interpolated Cumberland River stage and 24-hr precipitation. Note 1.00 $f t=0.305 \mathrm{~m}$ and $1.00 \mathrm{in}=2.54 \mathrm{~cm}$.

In the riparian zone, lateral subsurface flow predominates, with overland flow and groundwater recharge being secondary. Lateral flow assumptions are premised on the transmissivity feedback concept (runoff reaches streams as shallow subsurface flow rather than overland flow) and entail Darcian flow, time-invariant hydraulic gradient, and spatially homogeneous discharge rates (Grabs et al., 2012). Watson et al. (2018) investigated lateral flow within the riparian zone using temperature as an environmental tracer to delineate hyporheic and groundwater flow. Heat is transferred from the river to the riparian zone by advection at times of base flow. During peak flows, the flood pulse could be observed as lateral movement of a heat plume retreating from the river and returning with restored base flow. The time to return to preflood temperatures decreased 
with distance, as distal areas drain first, and thermal disturbances lasted days after flood waters receded. The distinct lateral movement of the heat plume indicates that flood waters laterally infiltrate and exit riparian zones.

In the current study, two instances of temperature changes with hydraulic-gradient reversals point to differing infiltration behaviors. Temperature in $\mathrm{MW}-7$ decreased $0.5^{\circ} \mathrm{C}$ during a 38-hr period April 22-24, 2017 (Figure 12). In contrast, on February 11, 2018, temperature fell $4.2^{\circ} \mathrm{C}$ in $1 \mathrm{hr}$ in $\mathrm{MW}-7$ and fell $3.5^{\circ} \mathrm{C}$ in $2 \mathrm{hr}$ in MW-6 (Figure 12). The first instance is suggestive of lateral infiltration, whereas the second, more dramatic instance indicates flood water overtopping the wells, resulting in short-circuit flow downward along the well casing, consistent with chloride results.

\subsubsection{Hydraulic Conductivity}

The 16 sets of sieve analyses were input into the program HydrogeoSieveXL to estimate hydraulic conductivity (K) based on 15 empirical relationships (Table 17). Five equations met the criteria for all samples: Sauerbrei, Zunker, Barr, Alyamani and Sen, and Krumbein and Monk. The geometric mean K ranged from $0.00186-0.0141 \mathrm{~cm} / \mathrm{s}$, while the arithmetic mean K ranged from $0.00210-0.148 \mathrm{~cm} / \mathrm{s}$. MW-7 and MW-9R had the lowest and highest mean $\mathrm{K}$ values, respectively. When compared to $\mathrm{K}$ ranges for unconsolidated sediments tabulated by Fetter (1994), the estimated K values fall within the expected magnitudes for silty sands, fine sands $\left(10^{-5}-10^{-3} \mathrm{~cm} / \mathrm{s}\right)$, and well-sorted sands $\left(10^{-3}-10^{-1}\right.$ $\mathrm{cm} / \mathrm{s}$ ). These lithologies are consistent with the soil core log descriptions and grain-size analysis results. The HydrogeoSieveXL reports are available in the appendices as HydrogeoSieveXL Hydraulic MW No. 
Table 17. HLDTC HydrogeoSieveXL Hydraulic Conductivity (K) Estimation

\begin{tabular}{|c|c|c|c|c|c|c|c|c|}
\hline Well ID & MW-1-3 & MW-3R-3 & MW-3R-4 & MW-4-3 & MW-4-4 & MW-4-5 & MW-5-4 & MW-5-5a \\
\hline Depth bls (m) & $2.9-4.4$ & $2.9-4.4$ & $4.4-5.9$ & $2.9-4.4$ & $4.4-5.9$ & $5.9-7.3$ & $4.4-5.9$ & $5.9-6.6$ \\
\hline Method (units) & $(\mathrm{cm} / \mathrm{s})$ & $(\mathrm{cm} / \mathrm{s})$ & $(\mathrm{cm} / \mathrm{s})$ & $(\mathrm{cm} / \mathrm{s})$ & $(\mathrm{cm} / \mathrm{s})$ & $(\mathrm{cm} / \mathrm{s})$ & $(\mathrm{cm} / \mathrm{s})$ & $(\mathrm{cm} / \mathrm{s})$ \\
\hline Hazen & $2.67 \mathrm{E}-03$ & $6.36 \mathrm{E}-03$ & $8.82 \mathrm{E}-03$ & $1.17 \mathrm{E}-02$ & $1.75 \mathrm{E}-02$ & 4.99E-03 & $8.97 \mathrm{E}-03$ & $9.31 \mathrm{E}-03$ \\
\hline Hazen* & $2.07 \mathrm{E}-03$ & 4.27E-03 & $5.72 \mathrm{E}-03$ & $7.68 \mathrm{E}-03$ & $1.11 \mathrm{E}-02$ & 3.39E-03 & 5.98E-03 & 6.19E-03 \\
\hline Slichter & 8.30E-04 & $2.26 \mathrm{E}-03$ & $3.25 \mathrm{E}-03$ & 4.29E-03 & $6.59 \mathrm{E}-03$ & $1.75 \mathrm{E}-03$ & $3.21 \mathrm{E}-03$ & 3.34E-03 \\
\hline Terzaghi & $1.44 \mathrm{E}-03$ & $3.96 \mathrm{E}-03$ & $5.71 \mathrm{E}-03$ & $7.54 \mathrm{E}-03$ & $1.16 \mathrm{E}-02$ & $3.07 \mathrm{E}-03$ & $5.63 \mathrm{E}-03$ & 5.87E-03 \\
\hline Beyer & $2.25 \mathrm{E}-03$ & $5.01 \mathrm{E}-03$ & $6.86 \mathrm{E}-03$ & $9.16 \mathrm{E}-03$ & $1.35 \mathrm{E}-02$ & $3.95 \mathrm{E}-03$ & $7.05 \mathrm{E}-03$ & $7.31 \mathrm{E}-03$ \\
\hline Sauerbrei & $2.55 \mathrm{E}-03$ & $6.42 \mathrm{E}-03$ & $8.51 \mathrm{E}-03$ & $1.03 \mathrm{E}-02$ & $1.34 \mathrm{E}-02$ & $4.83 \mathrm{E}-03$ & $8.70 \mathrm{E}-03$ & $8.96 \mathrm{E}-03$ \\
\hline Kruger & $4.66 \mathrm{E}-03$ & 6.92E-03 & $8.47 \mathrm{E}-03$ & $1.18 \mathrm{E}-02$ & $1.59 \mathrm{E}-02$ & $5.82 \mathrm{E}-03$ & $9.46 \mathrm{E}-03$ & $9.75 \mathrm{E}-03$ \\
\hline Kozeny-Carmen & $5.54 \mathrm{E}-03$ & $1.17 \mathrm{E}-02$ & $1.64 \mathrm{E}-02$ & $2.35 \mathrm{E}-02$ & $3.48 \mathrm{E}-02$ & $9.40 \mathrm{E}-03$ & $1.70 \mathrm{E}-02$ & $1.77 \mathrm{E}-02$ \\
\hline Zunker & $3.66 \mathrm{E}-03$ & $6.69 \mathrm{E}-03$ & $8.89 \mathrm{E}-03$ & $1.26 \mathrm{E}-02$ & $1.80 \mathrm{E}-02$ & $5.47 \mathrm{E}-03$ & $9.50 \mathrm{E}-03$ & $9.85 \mathrm{E}-03$ \\
\hline Zamarin & $5.23 \mathrm{E}-03$ & 8.41E-03 & $1.06 \mathrm{E}-02$ & $1.49 \mathrm{E}-02$ & $2.04 \mathrm{E}-02$ & 7.01E-03 & $1.17 \mathrm{E}-02$ & $1.20 \mathrm{E}-02$ \\
\hline USBR & $1.32 \mathrm{E}-03$ & $2.37 \mathrm{E}-03$ & $2.77 \mathrm{E}-03$ & $3.40 \mathrm{E}-03$ & $4.31 \mathrm{E}-03$ & $1.81 \mathrm{E}-03$ & $2.97 \mathrm{E}-03$ & $3.04 \mathrm{E}-03$ \\
\hline Barr & $1.14 \mathrm{E}-03$ & $3.37 \mathrm{E}-03$ & $4.96 \mathrm{E}-03$ & $6.52 \mathrm{E}-03$ & $1.02 \mathrm{E}-02$ & $2.59 \mathrm{E}-03$ & $4.81 \mathrm{E}-03$ & $5.02 \mathrm{E}-03$ \\
\hline Alyamani and Sen & $8.40 \mathrm{E}-04$ & $3.19 \mathrm{E}-03$ & $4.92 \mathrm{E}-03$ & $6.31 \mathrm{E}-03$ & $1.03 \mathrm{E}-02$ & $2.53 \mathrm{E}-03$ & $4.52 \mathrm{E}-03$ & 4.74E-03 \\
\hline Chapuis & $9.31 \mathrm{E}-04$ & $4.19 \mathrm{E}-03$ & $6.92 \mathrm{E}-03$ & $9.16 \mathrm{E}-03$ & $1.58 \mathrm{E}-02$ & $3.05 \mathrm{E}-03$ & $6.29 \mathrm{E}-03$ & $6.65 \mathrm{E}-03$ \\
\hline Krumbein and Monk & $2.74 \mathrm{E}-03$ & $4.36 \mathrm{E}-03$ & $5.46 \mathrm{E}-03$ & $7.24 \mathrm{E}-03$ & $9.41 \mathrm{E}-03$ & $3.50 \mathrm{E}-03$ & $5.78 \mathrm{E}-03$ & $5.98 \mathrm{E}-03$ \\
\hline Geometric Mean & $1.89 \mathrm{E}-03$ & $4.65 \mathrm{E}-03$ & $6.41 \mathrm{E}-03$ & $8.41 \mathrm{E}-03$ & $1.18 \mathrm{E}-02$ & $3.60 \mathrm{E}-03$ & $6.47 \mathrm{E}-03$ & $6.72 \mathrm{E}-03$ \\
\hline Arithmetic Mean & $2.18 \mathrm{E}-03$ & 4.84E-03 & $6.60 \mathrm{E}-03$ & $8.69 \mathrm{E}-03$ & $1.24 \mathrm{E}-02$ & $3.79 \mathrm{E}-03$ & $6.73 \mathrm{E}-03$ & $6.98 \mathrm{E}-03$ \\
\hline
\end{tabular}


Table 17. HLDTC HydrogeoSieveXL Hydraulic Conductivity (K) Estimation (cont.)

\begin{tabular}{|c|c|c|c|c|c|c|c|c|}
\hline Well ID & MW-5-5b & MW-5-6b & MW-7-5 & MW-7-7b & MW-8-2 & MW-9R-2 & MW-9R-3 & MW-9R-4 \\
\hline $\begin{array}{l}\text { Depth bls (m) } \\
\text { Method (units) }\end{array}$ & $\begin{array}{l}6.6-7.3 \\
(\mathrm{~cm} / \mathrm{s})\end{array}$ & $\begin{array}{l}7.3-8.8 \\
(\mathrm{~cm} / \mathrm{s})\end{array}$ & $\begin{array}{l}5.9-7.3 \\
(\mathrm{~cm} / \mathrm{s})\end{array}$ & $\begin{array}{l}8.8-10.2 \\
(\mathrm{~cm} / \mathrm{s})\end{array}$ & $\begin{array}{l}1.5-2.9 \\
(\mathrm{~cm} / \mathrm{s})\end{array}$ & $\begin{array}{l}1.5-2.9 \\
(\mathrm{~cm} / \mathrm{s})\end{array}$ & $\begin{array}{l}2.9-4.4 \\
(\mathrm{~cm} / \mathrm{s})\end{array}$ & $\begin{array}{l}4.4-5.9 \\
(\mathrm{~cm} / \mathrm{s})\end{array}$ \\
\hline Hazen & $7.00 \mathrm{E}-03$ & $8.48 \mathrm{E}-03$ & $2.49 \mathrm{E}-03$ & $4.49 \mathrm{E}-03$ & $1.59 \mathrm{E}-02$ & $1.49 \mathrm{E}-02$ & $1.05 \mathrm{E}-02$ & $2.00 \mathrm{E}-02$ \\
\hline Hazen* & $4.81 \mathrm{E}-03$ & $5.75 \mathrm{E}-03$ & $1.85 \mathrm{E}-03$ & $3.08 \mathrm{E}-03$ & $1.02 \mathrm{E}-02$ & $9.53 \mathrm{E}-03$ & $6.82 \mathrm{E}-03$ & $1.22 \mathrm{E}-02$ \\
\hline Slichter & $2.43 \mathrm{E}-03$ & $2.98 \mathrm{E}-03$ & $8.05 \mathrm{E}-04$ & $1.56 \mathrm{E}-03$ & $5.93 \mathrm{E}-03$ & $5.53 \mathrm{E}-03$ & $3.87 \mathrm{E}-03$ & $7.84 \mathrm{E}-03$ \\
\hline Terzaghi & $4.26 \mathrm{E}-03$ & $5.23 \mathrm{E}-03$ & $1.40 \mathrm{E}-03$ & $2.73 \mathrm{E}-03$ & $1.04 \mathrm{E}-02$ & $9.73 \mathrm{E}-03$ & $6.81 \mathrm{E}-03$ & $1.38 \mathrm{E}-02$ \\
\hline Beyer & $5.56 \mathrm{E}-03$ & $6.70 \mathrm{E}-03$ & $2.05 \mathrm{E}-03$ & $3.57 \mathrm{E}-03$ & $1.24 \mathrm{E}-02$ & $1.15 \mathrm{E}-02$ & $8.18 \mathrm{E}-03$ & $1.53 \mathrm{E}-02$ \\
\hline Sauerbrei & 7.03E-03 & 8.34E-03 & $2.57 \mathrm{E}-03$ & 4.28E-03 & $1.26 \mathrm{E}-02$ & $1.21 \mathrm{E}-02$ & $9.71 \mathrm{E}-03$ & $1.55 \mathrm{E}-02$ \\
\hline Kruger & $8.14 \mathrm{E}-03$ & $9.46 \mathrm{E}-03$ & $4.06 \mathrm{E}-03$ & $5.43 \mathrm{E}-03$ & $1.60 \mathrm{E}-02$ & $1.38 \mathrm{E}-02$ & $9.98 \mathrm{E}-03$ & $1.79 \mathrm{E}-02$ \\
\hline Kozeny-Carmen & $1.32 \mathrm{E}-02$ & $1.64 \mathrm{E}-02$ & $5.14 \mathrm{E}-03$ & $8.52 \mathrm{E}-03$ & $3.27 \mathrm{E}-02$ & $2.83 \mathrm{E}-02$ & $1.92 \mathrm{E}-02$ & $5.17 \mathrm{E}-02$ \\
\hline Zunker & $7.70 \mathrm{E}-03$ & $9.32 \mathrm{E}-03$ & $3.30 \mathrm{E}-03$ & $5.02 \mathrm{E}-03$ & $1.74 \mathrm{E}-02$ & $1.50 \mathrm{E}-02$ & $1.04 \mathrm{E}-02$ & $2.40 \mathrm{E}-02$ \\
\hline Zamarin & 9.83E-03 & $1.16 \mathrm{E}-02$ & 4.62E-03 & $6.50 \mathrm{E}-03$ & $2.02 \mathrm{E}-02$ & $1.74 \mathrm{E}-02$ & $1.25 \mathrm{E}-02$ & $2.44 \mathrm{E}-02$ \\
\hline USBR & $2.78 \mathrm{E}-03$ & 2.99E-03 & $1.17 \mathrm{E}-03$ & & 4.19E-03 & $3.90 \mathrm{E}-03$ & $3.06 \mathrm{E}-03$ & $4.35 \mathrm{E}-03$ \\
\hline Barr & $3.57 \mathrm{E}-03$ & $4.42 \mathrm{E}-03$ & $1.13 \mathrm{E}-03$ & $2.29 \mathrm{E}-03$ & $9.11 \mathrm{E}-03$ & $8.52 \mathrm{E}-03$ & $5.91 \mathrm{E}-03$ & $1.25 \mathrm{E}-02$ \\
\hline Alyamani and Sen & $3.18 \mathrm{E}-03$ & 4.04E-03 & $8.96 \mathrm{E}-04$ & $2.17 \mathrm{E}-03$ & $9.04 \mathrm{E}-03$ & $8.46 \mathrm{E}-03$ & $5.81 \mathrm{E}-03$ & $1.30 \mathrm{E}-02$ \\
\hline Chapuis & 4.28E-03 & $5.56 \mathrm{E}-03$ & 9.97E-04 & 2.60E-03 & $1.37 \mathrm{E}-02$ & $1.27 \mathrm{E}-02$ & 8.37E-03 & $2.13 \mathrm{E}-02$ \\
\hline Krumbein and Monk & 4.99E-03 & $5.78 \mathrm{E}-03$ & $2.59 \mathrm{E}-03$ & $3.47 \mathrm{E}-03$ & $9.78 \mathrm{E}-03$ & $8.24 \mathrm{E}-03$ & $6.40 \mathrm{E}-03$ & $1.06 \mathrm{E}-02$ \\
\hline Geometric Mean & $5.07 \mathrm{E}-03$ & $6.14 \mathrm{E}-03$ & $1.86 \mathrm{E}-03$ & $3.27 \mathrm{E}-03$ & $1.09 \mathrm{E}-02$ & $1.04 \mathrm{E}-02$ & $7.53 \mathrm{E}-03$ & $1.41 \mathrm{E}-02$ \\
\hline Arithmetic Mean & $5.34 \mathrm{E}-03$ & $6.43 \mathrm{E}-03$ & $2.10 \mathrm{E}-03$ & $3.45 \mathrm{E}-03$ & $1.15 \mathrm{E}-02$ & $1.06 \mathrm{E}-02$ & $7.74 \mathrm{E}-03$ & $1.48 \mathrm{E}-02$ \\
\hline
\end{tabular}
Notes:

blue Did not meet criteria

* $\mathrm{d}_{10}(\mathrm{~mm})$ 
Results of hydraulic tests completed in March 2018 are presented in Table 18. Hydraulic conductivity values were calculated via the Bouwer and Rice, Hvorslev, and Kansas Geological Survey (no skin) methods for slug tests, while the pumping tests were analyzed using the Theis method for a partially penetrating well in an unconfined aquifer. Mean $\mathrm{K}$ values for slug tests ranged from $4.85 \times 10^{-6}-1.00 \times 10^{-3} \mathrm{~cm} / \mathrm{s}$ (geometric) and $3.27 \times 10^{-6}-1.10 \times 10^{-3} \mathrm{~cm} / \mathrm{s}$ (arithmetic). MW-1 and MW-11 had the highest and lowest K values, respectively. Pumping-test K values were $4.83 \times 10^{-4} \mathrm{~cm} / \mathrm{s}$ for MW-6, $1.92 \times 10^{-5}$ $\mathrm{cm} / \mathrm{s}$ for MW-7, and $5.72 \times 10^{-6} \mathrm{~cm} / \mathrm{s}$ for MW-5. Broadly speaking, spatial trends in $\mathrm{K}$ are consistent with textural trends observed in cores. Wells located closer to the river (MW-11 and MW-7), where the alluvium is relatively fine-grained, exhibited the lowest $\mathrm{K}$ values, whereas wells close to the base of the ridge (MW-1 and MW-6) exhibited relatively high K values. When compared to the K ranges for unconsolidated sediments in Fetter (1994), the estimated $\mathrm{K}$ values fall within the expected magnitudes for silt, sandy silts, clayey sands $\left(10^{-6}-10^{-4} \mathrm{~cm} / \mathrm{s}\right)$, silty sands, fine sands $\left(10^{-5}-10^{-3} \mathrm{~cm} / \mathrm{s}\right)$, and well-sorted sands $\left(10^{-3}-10^{-1}\right.$ $\mathrm{cm} / \mathrm{s})$.

Previous studies of groundwater flow within $100 \mathrm{~km}$ of HLDTC included a component of K testing via pressure injection tests. Similar to the water chemistry data comparison, comparison of $\mathrm{K}$ data focused on individual wells that were representative of HLDTC site conditions (completed within Breathitt Fm., located in alluvium or weathered sandstones, similar total depths). Hydraulic conductivity results, as summarized by Minns (1993), were $2.03 \times 10^{-7} \mathrm{~cm} / \mathrm{s}$ to $3.05 \times 10^{-6} \mathrm{~cm} / \mathrm{s}$ (Kipp and Dinger, 1987); $2.13 \times 10^{-6} \mathrm{~cm} / \mathrm{s}$ (Wunsch, 1992); $3.5 \times 10^{-7} \mathrm{~cm} / \mathrm{s}$ to $9.3 \times 10^{-4} \mathrm{~cm} / \mathrm{s}$ (Wunsch, 1993); and $1.02 \times 10^{-7}$ to $1.52 \times 10^{-5} \mathrm{~cm} / \mathrm{s}$, mean $5.1 \times 10^{-6} \mathrm{~cm} / \mathrm{s}$ and median $3.56 \times 10^{-7} \mathrm{~cm} / \mathrm{s}$ (Minns, 1993). These 
values overlap the range of $\mathrm{K}$ values obtained by pump and slug hydraulic conductivity testing at HLDTC (Table 18). The fact that maximum values of $\mathrm{K}$ in this study are larger than in the other studies cited is consistent with wells being completed primarily in alluvium at HLDTC. 
Table 18. HLDTC Well Capacity Hydraulic Conductivity Values, Slug and Pump Tests

\begin{tabular}{|c|c|c|c|c|c|c|c|c|c|}
\hline $\begin{array}{l}\text { Well ID } \\
\text { Method } \\
\text { (units) }\end{array}$ & $\begin{array}{l}\text { MW-1 } \\
(\mathrm{cm} / \mathrm{s}) \\
\end{array}$ & $\begin{array}{l}\text { MW-2 } \\
(\mathrm{cm} / \mathrm{s}) \\
\end{array}$ & $\begin{array}{l}\text { MW-4 } \\
(\mathrm{cm} / \mathrm{s}) \\
\end{array}$ & $\begin{array}{l}\text { MW-5 } \\
(\mathrm{cm} / \mathrm{s}) \\
\end{array}$ & $\begin{array}{l}\text { MW-6 } \\
(\mathrm{cm} / \mathrm{s}) \\
\end{array}$ & $\begin{array}{l}\text { MW-7 } \\
(\mathrm{cm} / \mathrm{s}) \\
\end{array}$ & $\begin{array}{l}\text { MW-8 } \\
(\mathrm{cm} / \mathrm{s})\end{array}$ & $\begin{array}{l}\text { MW-10R } \\
(\mathrm{cm} / \mathrm{s})\end{array}$ & $\begin{array}{l}\text { MW-11 } \\
(\mathrm{cm} / \mathrm{s})\end{array}$ \\
\hline Bouwer \& & & & & & & & & & \\
\hline Rice & $8.27 \mathrm{E}-04$ & $1.40 \mathrm{E}-05$ & $4.57 \mathrm{E}-05$ & -- & -- & -- & $9.82 \mathrm{E}-05$ & $3.20 \mathrm{E}-04$ & $4.18 \mathrm{E}-06$ \\
\hline Hvorslev & $1.17 \mathrm{E}-03$ & $1.87 \mathrm{E}-05$ & $5.50 \mathrm{E}-05$ & -- & -- & -- & $1.40 \mathrm{E}-04$ & $4.15 \mathrm{E}-04$ & $5.62 \mathrm{E}-06$ \\
\hline $\begin{array}{l}\text { Kansas GS } \\
\text { (no skin) }\end{array}$ & $1.04 \mathrm{E}-03$ & $3.82 \mathrm{E}-06$ & $3.25 \mathrm{E}-03$ & -- & -- & -- & $1.27 \mathrm{E}-04$ & $4.07 \mathrm{E}-04$ & -- \\
\hline $\begin{array}{l}\text { Theis* } \\
\text { Geometric }\end{array}$ & -- & -- & -- & $5.72 \mathrm{E}-06$ & 4.83E-04 & $1.92 \mathrm{E}-05$ & -- & -- & -- \\
\hline $\begin{array}{l}\text { Mean } \\
\text { Arithmetic }\end{array}$ & $1.00 \mathrm{E}-03$ & $1.00 \mathrm{E}-05$ & 4.69E-05 & -- & -- & -- & $1.20 \mathrm{E}-04$ & $3.78 \mathrm{E}-04$ & $4.85 \mathrm{E}-06$ \\
\hline Mean & $1.01 \mathrm{E}-03$ & $1.22 \mathrm{E}-05$ & 4.72E-05 & -- & -- & -- & $1.22 \mathrm{E}-04$ & $3.81 \mathrm{E}-04$ & $3.27 \mathrm{E}-06$ \\
\hline
\end{tabular}


Groundwater velocity ranges were calculated along three transects from the base of the slope to near the Cumberland River: MW-6 to MW-7, MW-4 to MW-5, and MW-1 to MW-2. The groundwater velocity equation (from Darcy's law) is:

$$
v=-K / n\left(\frac{\Delta h}{\Delta l}\right)
$$

where $\mathrm{v}$ is velocity, $\mathrm{K}$ is hydraulic conductivity (Table 18, geometric mean or Theis [MW6 and MW-7]), $\mathrm{n}$ is total porosity as calculated by HydrogeoSieveXL (or, if unavailable, the site mean $\mathrm{n}[0.408]), \Delta \mathrm{h}$ is change in hydraulic head (mean water levels, Table 3 ), and $\Delta \mathrm{l}$ is the distance between wells. Velocity ranges overlapped, but were highest along transect MW-1 to MW-2 $(0.0057-0.5027 \mathrm{~cm} / \mathrm{s})$, intermediate along MW-6 to MW-7 $(0.0050-0.1215 \mathrm{~cm} / \mathrm{s})$, and lowest along MW-4 to MW-5 $(0.0021-0.0168 \mathrm{~cm} / \mathrm{s})$. The differences in calculated groundwater velocities reflect differences in topographic gradient and soil texture.

\section{Conclusions and Recommendations}

\subsection{Conclusions}

Based on the MOA, this study has addressed five objectives: mapping the water table across the site; determining seasonal fluctuations in the depth of the water table; defining directions and rates of groundwater flow; assessing groundwater interactions with the Cumberland River; and characterizing seasonal variations in groundwater and surface water chemistry, including consideration of land use and training activities on water quality. Depth to the water table ranged from $0.83 \mathrm{~m}$ bls (in MW-8 in January and April 2017) to $5.76 \mathrm{~m}$ bls (in MW-10R in January 2017 and MW-7 in October 2017). The water table was generally shallowest in January 2017 and deepest in April 2017. The hydraulic 
head tended to decrease with distance away from the base of the ridge and toward the Cumberland River, consistent with groundwater flow toward the river as regional base level. A downward hydraulic gradient from the alluvium to bedrock is evident near the base of the ridge in the south-central part of the site, but based on the limits of this study, it is not clear if downward groundwater flow exists between the aquifers. Soil texture tended to become finer and values of hydraulic conductivity tended to decrease with proximity to the river. Occasional river-stage rises resulted in hydraulic gradient reversals that lasted as long as 8-10 days (in February 2018). During that flood, inundation of monitoring wells MW-6 and MW-7 in the western part of the site was indicated by watertemperature declines of several degrees Celsius and by elevated chloride concentrations measured during pumping tests in March 2018. In conjunction with water-level data, stable isotope results suggest that recharge occurs primarily from late fall through early spring and that diffuse infiltration from land surface to top of bedrock takes several months. Groundwater beneath the site does not appear to be impacted by current land use (farming, military training, and natural gas production), as indicated by generally low concentrations of metals (other than iron), nitrogen compounds, VOCs, and anions (i.e., salinity).

\subsection{Recommendations}

Following discussions with KNG personnel, two of the 11 monitoring wells (MW3R and MW-6) installed during this study were not abandoned. MW-6 has been incorporated into the KGS statewide groundwater monitoring network. Both wells can be used with a "trash" pump designed to fit into 2-in. PVC to meet light to moderate wateruse demands. In particular, water-table drawdown at MW-6 appeared to stabilize at a pumping rate of $1.3 \mathrm{~L} / \mathrm{min}(1900 \mathrm{~L} /$ day $)$ during aquifer testing in March 2018, consistent 
with yields from alluvial wells cited by Kilburn et al. (1962). Both wells met established EPA drinking-water standards with the exception of elevated levels of $\mathrm{Fe}$ and $\mathrm{Mn}$, which are secondary (aesthetic) exceedances, and As on one occasion (January 5, 2018). Treatment technologies such as aeration and filtration are available for limiting $\mathrm{Fe}, \mathrm{Mn}$, and As concentrations. Ecological impacts due to groundwater withdrawal were not assessed as part of this study and should be considered due to the proximity of the wetland to MW-6. 


\section{References}

Army National Guard, Harold L. Disney Training Center (ANG, HLDTC). Accessed December 2016. http://whfrtc.ky.gov/hldtc/Pages/default.aspx

Aseltyne, T.A., Rowe, H.D., and Fryar, A.E., 2006. Stable isotopic fingerprint of a hyporheic-hypolentic boundary in a reservoir. Hydrogeology Journal, v. 14, 1688-1695.

Barnes, C.J., and Allison, G.B., 1988. Tracing of water movement in the unsaturated zone using stable isotopes of hydrogen and oxygen. Journal of Hydrology, v. 100, no. 1-3, 143176.

Bauluz, B., Mayayo, M.J., Fernandez-Nieto, C., and Gonzalez Lopez, J.M. 2000. Geochemistry of Precambrian and Paleozoic siliciclastic rocks from the Iberian Range NE Spain: implications for source-area weathering, sorting, provenance, and tectonic setting. Chemical Geology, v. 168, 135-150.

Bhatia, M.R., 1983. Plate tectonics and geochemical composition of sandstones. Journal of Geology, v. 91, no. 6, 611-627.

Brookfield, A.E., Stotler, R.L., and Reboulet, E.C., 2017. Interpreting temporal variations in river response functions: an example from the Arkansas River, Kansas, USA. Hydrogeology Journal, v. 25, 1271-1282.

Carey, D.I., and Stickney, J.F., 2005. Groundwater resources of Knox County, Kentucky. Kentucky Geological Survey, University of Kentucky. Ser. XII, County Report 61.

Catto, N.R., and Quaternary Research Group, 1989. Geology 482: field and laboratory manual. Edmonton: University of Alberta, Dept. of Geology.

CH2M Hill, 1992, Results of the site investigation, Phase II, at the Paducah Gaseous Diffusion Plant, Paducah, Kentucky. Report KY/SUB/13B-97777C P-03/1991/1, prepared for the U.S. Department of Energy under subcontract to DOE contract DE-AC0576OR00001. Oak Ridge, Tennessee: CH2M Hill.

Chesnut, D.R., Jr., 1992. Stratigraphic and structural framework of the Carboniferous rocks of the Central Appalachian Basin in Kentucky. Kentucky Geological Survey, Ser. XI, Bulletin 3, 42 p.

Cronan, C.S., Piampiano, J.T., and Patterson, H.H., 1999. Landscape and watershed processes: Influence of land use and hydrology of exports of carbon and nitrogen in a Maine river basin. Journal of Environmental Quality, v. 28, 953-961.

Cullers, R.L., Basu, A., and Suttner, L.J., 1988. Geochemical signature of provenance in sand-size material in soils and stream sediments near the Tobacco Root Batholith, Montana, U.S.A. Chemical Geology, v. 70, 335-348. 
Cumbie, D.H., Galceran, Jr., C., Dinger, J.S., and Webb, S.E., 2004. Evaluation of water quality at the Kentucky Army National Guard Artemus Training Site, Knox County. Kentucky Geological Survey, contract report to Kentucky Department of Military Affairs. $26 \mathrm{pp}$.

Devlin, J.F., 2015. HydrogeoSieveXL: an Excel-based tool to estimate hydraulic conductivity from grain size analysis. Hydrogeology Journal, v. 23, no. 4, 837-844.

Dickinson, W.R., and Suczek, C.A., 1979. Plate tectonics and sandstone compositions. American Association of Petroleum Geologists Bulletin, v. 63, no. 12, 2164-2182.

Federal Emergency Management Agency, 2015. Flood insurance rate map, Knox County, Kentucky and incorporated areas. Panel 255 of 350. Map No. 21121C0255F.

Fetter, C.W., 1994. Applied Hydrogeology, 3rd edition. New York: Macmillan.

Fiebig, D.M., Lock, M.A., and Neal, C., 1990. Soil water in the riparian zone as a source of carbon for a headwater stream. Journal of Hydrology, v. 116, 217-237.

Fritz, S.J., 1994. A survey of charge-balance errors on published analyses of potable ground and surface waters. Ground Water, v. 32, no. 4, 539-546.

Galceran, C.M., and Dinger, J.S., 2001. Water quality survey for Kentucky Army National Guard training sites. Kentucky Geological Survey, contract report to Kentucky Department of Military Affairs. 40 pp.

Gat, J.R., 1996. Oxygen and hydrogen isotopes in the hydrologic cycle. Annual Review of Earth and Planetary Sciences, v. 24, 225-262.

Grabs, T., Bishop, K., Laudon, H., Lyon, S.W., and Seibert, J. 2012. Riparian zone hydrology and soil water total organic carbon (TOC): implications for spatial variability and upscaling of lateral riparian TOC exports. Biogeosciences, v. 9, 3901-3916.

Haile, E., and Fryar, A.E., 2017. Chemical evolution of groundwater in the Wilcox aquifer of the northern Gulf Coastal Plain, USA. Hydrogeology Journal, v. 25, 2403-2418.

Herron, M.M. 1988. Geochemical classification of terrigenous sands and shales from core $\log$ data. Journal of Sedimentary Petrology, v. 58, no. 5, 820-829.

Hopkins, H.T., 1966. Fresh-saline interface map of Kentucky. U.S. Geological Survey, scale 1 in $=8 \mathrm{mi}$.

Kilburn, C., Price, Jr., W.E., and Mull, D.S., 1962. Availability of ground water in Bell, Clay, Jackson, Knox, Laurel, Leslie, McCreary, Owsley, Rockcastle, and Whitley Counties, Kentucky. U.S. Geological Survey Hydrologic Investigation Atlas HA-38. 4 sheets. 
Kipp, J.A., and Dinger, J.S, 1991. Stress-relief fracture control of ground-water movement in the Appalachian Plateaus. Kentucky Geological Survey, Ser. 11, Reprint 30, 11 p.

Kipp, J.A., Lawrence, F.N., and Dinger, J.S., 1983. A conceptual model of ground water flow in the Eastern Kentucky Coal Field: Symposium on Surface Mining, Hydrology, and Reclamation, 1983, University of Kentucky, Lexington, Kentucky, 543-548.

Lapham, W.W., Wilde, F.D., and Koterba, M.T., 1995. Ground-water data-collection protocols and procedures for the National Water-Quality Assessment Program: selection, installation, and documentation of wells, and collection of related data. U.S. Geological Survey Open-File Report 95-398, 26 pp.

Lyon, S.W., Grabs, T., Laudon, H., Bishop, K.H., and Seibert, J., 2011. Variability of groundwater levels and total organic carbon in the riparian zone of a boreal catchment. Journal of Geophysical Research: Biogeosciences, v. 116, no. G1, 1-12. DOI 10.1029/2010JG001452.

Maharjan, M., and Donovan, J.J., 2016. Groundwater response to serial stream stage fluctuations in shallow unconfined alluvial aquifers along a regulated stream (West Virginia, USA). Hydrogeology Journal, v. 24, 2003-2015.

McCoy, K.J., Yager, R.M., Nelms, D.L., Ladd, D.E., Monti, J., Jr., and Kozar, M.D., 2015. Hydrologic budget and conditions of Permian, Pennsylvanian, and Mississippian aquifers in the Appalachian Plateaus Physiographic Province. U.S. Geological Survey Scientific Investigations Report 2015-5106, 77 p.

McMordie Stoughton, K., Williamson, J., Boyd, B., Cabe, J., Brown, S., Dixon, D., Loper, S., Elliott, D., Wendel, E., De La Rosa, M., Giever, E., and Russell, B., 2013. Army Net Zero water balance and roadmap programmatic summary. Report prepared by Pacific Northwest National Laboratory for the United States Army. 56 p.

Minasny, B., Stockmann, U., Hartemink, A.E., and McBratney, A.B., 2016. Measuring and modelling soil depth functions. In: Hartemink, A., and Minasny, B. (eds). Digital Soil Morphometrics. Progress in Soil Science. Basel, Switzerland: Springer International Publishing Switzerland.

Minns, S.A., 1993. Conceptual model of local and regional ground-water flow in the eastern Kentucky coal field. Kentucky Geological Survey, Thesis Series 6, Series XI, 194 p.

Moumouni, A., and Fryar, A.E., 2017. Controls on groundwater quality and dug-well asphyxiation hazard in Dakoro area of Niger. Groundwater for Sustainable Development, v. 5, 235-243. 
Ohtani, T., Mizuno, T., Kouda, A., Kojima, S., 2015. Seasonal change of underground temperature and the use of geothermal in the Gifu City, Japan. Proceedings World Geothermal Congress 2015. Melbourne, Australia, 19-25 April 2015. 7 p.

Parkhurst, D.L., and Appelo, C.A.J., 2013. Description of input and examples for PHREEQC version 3: a computer program for speciation, batch-reaction, one-dimensional transport, and inverse geochemical calculations. U.S. Geological Survey Techniques and Methods 6-A43, $497 \mathrm{p}$.

Powell, J.D., and Larson, J.D., 1985. Relation between ground-water quality and mineralogy in the coal-producing Norton Formation of Buchanan County, Virginia. U.S. Geological Survey Water-Supply Paper 2274, 21 p.

Price, J.R., and Velbel, M.A., 2002. Chemical weathering indices applied to weathering profiles developed on heterogeneous felsic metamorphic parent rocks. Chemical Geology, v. 202, 397-416.

Price, W.E., Jr., D.S., Mull, D.S., and Kilburn, C., 1962. Reconnaissance of ground-water resources in the eastern coal field region, Kentucky. U.S. Geological Survey Water-Supply Paper 1607, $56 \mathrm{p}$.

Rice, D.D., 1974. Geologic map of Artemus Quadrangle, Bell and Knox Counties, Kentucky. U.S. Geological Survey Map GQ-1207.

Risser, D.W., Thompson, R.E., and Stuckey, M.H., 2008. Regression method for estimating long-term mean annual ground-water recharge rates from base flow in Pennsylvania. U.S. Geological Survey Scientific Investigations Report 2008-5185, 23 p.

Ruxton, B.P., 1968. Measures of the degree of chemical weathering of rocks. Journal of Geology, v. 76, no. 5, 518-527.

Schmidt, G.W., 1973. Interstitial water composition and geochemistry of deep Gulf Coast shales and sandstones. American Association of Petroleum Geologists Bulletin, v. 57, no. $2,321-337$.

Schweitzer, P., 2015. Geologic units in Knox County, Kentucky. USGS, Mineral Resources On-Line Spatial Data. Accessed February 2017. https://mrdata.usgs.gov/geology/state/fips-unit.php?code=f21121

Sharp, Z., 2017. Principles of Stable Isotope Geochemistry, 2nd Edition. Upper Saddle River, New Jersey: Pearson/Prentice Hall. DOI 10.5072/FK2GB24S9F.

Sherratt, D., compiler, 2013. Disney Training Center, Artemus, Kentucky, Soil Type and Agricultural Lease Area. Kentucky National Guard. Frankfort, Kentucky, 1 sheet. 
Sherratt, D., compiler, undated. Disney Training Site Artemus, Kentucky. Kentucky National Guard. Frankfort, Kentucky, 1 sheet.

Snyder, K.M., and Sendlein, L.V.A., 1997. Artemus Training Site, Knox County, Kentucky, integrated natural resources plan, TR-0197. Kentucky Water Resources Research Institute. Lexington, Kentucky.

United States Army Corps of Engineers, Nashville District (USACE LRN). Nashville District river basins and boundaries. Accessed February 2017. http://www.lrn.usace.army.mil/portals/49/siteimages/Missions/Outreach/DistrictBoundar yMap.jpg

United States Environmental Protection Agency (EPA), 2000. Guidance for data quality assessment, practical methods for data analysis. EPA/600/R-96/084. Office of Environmental Information, Washington: DC.

United States Environmental Protection Agency (EPA), Region 3, 1991. Regional technical guidance manual, risk assessment. Regional guidance on handling chemical concentration data near the detection limit on risk assessments. Hazardous Waste Management Division, Office of Superfund Programs, Philadelphia: Pennsylvania.

USDA, 1994. Soil survey — Kentucky Army National Guard, Artemus Training Site, Knox County, Kentucky. USDA, Natural Resources Conservation Service and Knox County Conservation District. Lexington, Kentucky.

USDA, 1995. Resource inventory and conservation plan, Kentucky Army National Guard, Artemus Training Site, Knox County, Kentucky. USDA, Natural Resources Conservation Service and Knox County Conservation District. Lexington, Kentucky.

USDA, 2017. Custom soil resource report for Knox and eastern part of Whitley Counties, Kentucky for CSM Harold L. Disney. USDA, Natural Resources Conservation Service. 49 p.

Watson, J.A., Cardenas, M.B., Ferencz, S.B., Knappett, P.S.K., Neilson, B.T., 2018. The effects of floods on the temperature of riparian groundwater. Hydrological Processes, v. $32,1267-1281$.

Weatherbase. Artemus, Kentucky. Canty and Associates LLC. Accessed February 2017. http://www.weatherbase.com/weather/weatherall.php3? $\mathrm{s}=183051 \&$ cityname $=$ Artemus $\%$ $\underline{2 \mathrm{C}+\text { Kentucky } \% 2 \mathrm{C}+\text { United }+ \text { States }+ \text { of }+ \text { America\&units }}=$

Weinheimer, R.L., 1983. Vertical sequence and diagenesis in Breathitt sandstone of eastern Kentucky: M.S. Thesis, University of Cincinnati, Cincinnati, Ohio, 147 p. 
White, D., Palmer-Ball, B., Jr., and Laudermilk, E.L., 1995. Biological inventory: Eastern Kentucky Training Site-Artemus, Knox County, Kentucky. Kentucky State Nature Preserves Commission. Frankfort, Kentucky.

Wunsch, D.R., 1993. Ground-water geochemistry and its relationship to the flow system and unmined site in the eastern Kentucky coal field. Kentucky Geological Survey, Thesis Series 5, Series XI, $128 \mathrm{p}$.

Wyrick, G.C., and Borchers, J.W., 1981, Hydrologic effects of stress relief fractures on an Appalachian valley. U.S. Geological Survey Water-Supply Paper 2177, 51 p.

Zhang, Y., and Hartemink, A.E., 2018. Digital mapping of a soil profile. European Journal of Soil Science, v. 70, no. 1, 27-41 p. DOI 10.1111/ejss.12699.

Zogorski, J.S., Carter, J.M., Ivahnenko, T., Lapham, W.W, Moran, M.J., Rowe, B.L., Squillace, P.J., and Toccalino, P.L., 2006. The quality of our nation's waters, volatile organic compounds in the nation's ground water and drinking-water supply wells. U.S. Geological Survey Circular 1292, 7 p. 


\section{Vita}

\section{Amanda Rachelle Sherman}

\section{Education}

B.A. Chemistry; Minor, Geology (2018)

University of Kentucky

B.A. German; Minor, Marine Science (2003)

Eckerd College

\section{Experience}

Graduate Research and Teaching Assistant

Department of Earth and Environmental Sciences

University of Kentucky

Lexington, KY 40506

Environmental Restoration Manager, Physical Scientist and Biologist

Army Enviornmental Command

Joint Base San Antonio, TX 78234

Project Coordinator, Environmental Scientist and Biologist

HSA Engineers and Scientists

Tampa, FL 33617

High School Science Teacher

Admiral Farragut Academy

St. Petersburg, FL 33710 